Diaspore 16

\title{
Romance e Shoah
}

Pratiche di narrazione

sulla tragedia indicibile

Alessandro Cinquegrani, Francesca Pangallo, Federico Rigamonti 

Romance e Shoah

\section{Diaspore Quaderni di ricerca}

Collana diretta da $\mid$ A series edited by

Susanna Regazzoni

Ricciarda Ricorda

16

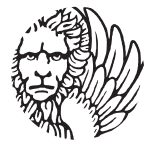

Edizioni

Ca'Foscari 


\title{
Diaspore Quaderni di ricerca
}

\author{
Direttori| General editors \\ Susanna Regazzoni (Università Ca' Foscari Venezia, Italia) \\ Ricciarda Ricorda (Università Ca' Foscari Venezia, Italia)
}

\section{Comitato scientifico|Advisory board}

Shaul Bassi (Università Ca' Foscari Venezia, Italia) Enric Bou (Università Ca' Foscari Venezia, Italia) Luisa Campuzano (Universidad de La Habana, Cuba) Ilaria Crotti (Università Ca' Foscari Venezia, Italia) Antonio Fernández Ferrer (Universidad de Alcalá, España) Monica Giachino (Università Ca' Foscari Venezia, Italia) Emilia Perassi (Università degli Studi di Milano, Italia) Eduardo Ramos Izquierdo (Université de Paris IV Sorbonne, France) Daniela Rizzi (Università Ca’ Foscari Venezia, Italia) Silvana Serafin (Università di Udine, Italia)

\section{Comitato di redazione | Editorial staff}

Margherita Cannavacciuolo (Università Ca' Foscari Venezia, Italia) Alberto Zava (Università Ca' Foscari Venezia, Italia)

\section{Lettori|Readers}

Rosanna Benacchio (Università degli Studi di Padova, Italia) Luis Fernando Beneduzi (Università Ca' Foscari Venezia, Italia) Anna Boschetti (Università Ca' Foscari Venezia, Italia) Silvia Camilotti (Università Ca' Foscari Venezia, Italia) Alessandro Cinquegrani (Università Ca' Foscari Venezia, Italia) Adriana Crolla (Universidad Nacional del Litoral, Argentina) Biagio D’Angelo (Universidade Federal do Rio Grande do Sul, Porto Alegre, Brasil) Alice Favaro (Università Ca' Foscari Venezia, Italia) Monica Giachino (Università Ca' Foscari Venezia, Italia) Marie Christine Jamet (Università Ca' Foscari Venezia, Italia) Adriana de los Angeles Mancini (Universidad de Buenos Aires, Argentina) Pia Masiero (Università Ca' Foscari Venezia, Italia) Maria del Valle Ojeda Calvo (Università Ca' Foscari Venezia, Italia) Patrizio Rigobon (Università Ca' Foscari Venezia, Italia) Michela Rusi (Università Ca' Foscari Venezia, Italia) Alessandro Scarsella (Università Ca' Foscari Venezia, Italia) María Carmen Simón Palmer (CSIC - Consejo Superior de Investigaciones Científicas, Madrid, España) Alessandra Trevisan (Università Ca' Foscari Venezia, Italia) Michela Vanon Alliata (Università Ca' Foscari Venezia, Italia)

\section{Direzione e redazione | Editorial office}

Università Ca' Foscari Venezia

Dipartimento di Studi Linguistici e Culturali Comparati

Ca'Bernardo

Dorsoduro, Calle Bernardo, 3199

30123 Venezia

$$
\begin{array}{rr}
\text { e-ISSN } & 2610-9387 \\
\text { ISSN } & 2610-8860
\end{array}
$$




\section{Romance e Shoah Pratiche di narrazione sulla tragedia indicibile}

Alessandro Cinquegrani, Francesca Pangallo, Federico Rigamonti

Venezia

Edizioni Ca' Foscari - Digital Publishing 2021 
Romance e Shoah. Pratiche di narrazione sulla tragedia indicibile

Alessandro Cinquegrani, Francesca Pangallo, Federico Rigamonti

(c) 2021 Alessandro Cinquegrani, Francesca Pangallo, Federico Rigamonti per il testo

(c) 2021 Edizioni Ca’ Foscari - Digital Publishing per la presente edizione

\section{(a) (1)}

Quest'opera è distribuita con Licenza Creative Commons Attribuzione 4.0 Internazionale. This work is licensed under a Creative Commons Attribution 4.0 International License.

OPEN

Qualunque parte di questa pubblicazione può essere riprodotta, memorizzata in un sistema di recupero dati o trasmessa in qualsiasi forma o con qualsiasi mezzo, elettronico o meccanico, senza autorizzazione, a condizione che se ne citi la fonte.

Any part of this publication may be reproduced, stored in a retrieval system, or transmitted in any form or by any means without permission provided that the source is fully credited.

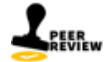

Certificazione scientifica delle Opere pubblicate da Edizioni Ca' Foscari - Digital Publishing: il saggio pubblicato ha ottenuto il parere favorevole da parte di valutatori esperti della materia, attraverso un processo di revisione doppia anonima, sotto la responsabilità del Comitato scientifico della collana. La valutazione è stata condotta in aderenza ai criteri scientifici ed editoriali di Edizioni Ca' Foscari, ricorrendo all'utilizzo di apposita piattaforma.

Scientific certification of the works published by Edizioni Ca' Foscari - Digital Publishing: the essay published has received a favourable evalutation by subject-matter experts, through a double blind peer review process under the responsibility of the Scientific Committee of the series. The evaluations were conducted in adherence to the scientific and editorial criteria established by Edizioni Ca' Foscari, using a dedicated platform.

Edizioni Ca' Foscari - Digital Publishing

Fondazione Università Ca' Foscari Venezia

Dorsoduro 3246, 30123 Venezia

http://edizionicafoscari.unive.it|ecf@unive.it

1a edizione aprile 2021

ISBN 978-88-6969-492-9 [ebook]

ISBN 978-88-6969-493-6 [print]

Pubblicato nell'ambito del Progetto d'Ateneo Modelli ermeneutici junghiani e postjunghiani dalla letteratura alle imprese.

Romance e Shoah. Pratiche di narrazione sulla tragedia indicibile / Alessandro Cinquegrani, Francesca Pangallo, Federico Rigamonti - 1. ed. - Venezia: Edizioni Ca' Foscari - Digital Publishing, 2021. - 194 p.; 23 cm. - (Diaspore; 16). - ISBN 978-88-6969-493-6.

URL https://edizionicafoscari.unive.it/it/edizioni/libri/978-88-6969-493-6/

DOI http://doi.org/10.30687/978-88-6969-492-9 
Romance e Shoah

Pratiche di narrazione sulla tragedia indicibile

Alessandro Cinquegrani, Francesca Pangallo, Federico Rigamonti

\section{Abstract}

Over the last 70 years, Holocaust representations increased significantly as cultural objects distributed on a large scale: fictional books, museum sites, artworks, documentaries, and films are only a few samples of those echoes the Holocaust produced in contemporary Western culture. There are some specific patterns in the way the Holocaust has been represented that, however, contrast with the survivors' account of the same event: for example, the dichotomy between bad and good characters so essential within Holocaust-based media - especially on television and film - does not really match with the testimony's experience. While storytelling strategies may help to involve the public by emotionally engaging with the story, the risks of altering the real meaning of the Holocaust are quite high: what we often label as a "story" is actually been an outrageous, documented mass-genocide. Furthermore, as the age gap between the present and the past generation progresses, also the collective awareness of Nazi crimes as a real fact gets compromised.

This volume explores selected Holocaust narrations by contextualizing the historical, literary, and social influences those texts had in their unique points of view. Starting with some recent examples of Holocaust exploitation through social media, the first chapter explores the paradigm shift when the Holocaust became a cultural, fictional trend rather than a historical massacre. In the second chapter, the analysis examines postmodern representations of Holocaust and Nazi semantics through relevant examples taken from both American and European literature. The third chapter analyses Europe Central by William T. Vollman, as all the narratological and cultural issues considered in the previous two chapters are well outlined in this articulated novel, where the relationship between reality and its representation after the postmodernist period is largely investigated. In chapter four, an account is given of the connections and differences between the narratological category romance, as understood by Northrop Frye, and Holocaust narration features. In chapter five, those elements are used to consider the work of Italian Holocaust survivor and Jewish writer Primo Levi, as his narration around Auschwitz adopts some fictional tools and still refuses undemanding storytelling mechanisms. The sixth and final chapter examines the relevant novel Les Benviellants by Jonathan Littell, considering its Nazi genocide account through the antagonist's perspective.

Keywords Holocaust. Romance. Postmodernism. Postmemory. Narratology. Ethics. Storytelling. Comparative Studies. Contemporary Literature. 



\section{Sommario}

Introduzione 9

1 La Shoah come oggetto culturale 19

2 Postmoderno e Shoah 47

3 Il caso Vollmann $\quad 75$

4 Romance e Shoah 109

5 Shoah oltre il romance: Primo Levi 131

6 Il caso Littell 159

$\begin{array}{ll}\text { Bibliografia } & 185\end{array}$ 



\section{Introduzione}

Sommario 1 Un fenomeno di massa. - 2 Romance. - 3 Struttura.

\section{Un fenomeno di massa}

Verso la fine dell'estate 2020, sulla piattaforma TikTok ha debuttato uno strano fenomeno di cosplay virtuale incentrato sull'esperienza concentrazionaria, il quale risulta essere affine alle tematiche di ricerca considerate nella presente sede d'indagine. Così viene raccontata sul magazine online Teen Vogue:

There's a new TikTok trend where users pretend to be Holocaust victims in heaven, complete with full makeup looks to simulate dark undereye circles, burns, and sunken cheeks. In the videos, users explain how they 'died', often saying that they were killed in the gas chambers at Auschwitz. Many of these videos inexplicably use Bruno Mars's pop song 'Locked Out of Heaven' as a soundtrack. ${ }^{1}$

Questo libro nasce da un'ampia e articolata riflessione comune, maturata durante lezioni e discussioni informali. Tuttavia, la stesura dei diversi capitoli è stata affidata a singoli autori come segue: «Introduzione»: paragrafo 1: Francesca Pangallo; paragrafi 2 e 3: Alessandro Cinquegrani; cap. 1, «La Shoah come oggetto culturale»: Francesca Pangallo; cap. 2, «Postmoderno e Shoah»: Alessandro Cinquegrani; cap. 3, «Il caso Vollmann»: Federico Rigamonti; cap. 4, «Romance e Shoah»: Alessandro Cinquegrani; cap. 5, «Shoah oltre il Romance: Primo Levi»: Francesca Pangallo; cap. 6, «Il caso Littell»: Federico Rigamonti.

1 K. Nesvig, «This "Holocaust” TikTok Challenge Is Rightfully Sparking Backlash». Teen Vogue, August 25, 2020, https://www.teenvogue.com/story/holocaust-tiktok-challenge-backlash; enfasi aggiunte. Il corsivo, salvo quando diversamente specificato, è nell'originale. 
Molti ragazzi di tutto il mondo, la cui età difficilmente supera i 20 anni, si sono sfidati in una competizione aperta sul social network TikTok fingendo di 'impersonare' vittime del genocidio nazista. Questa 'sfida' ha come base la dinamica di media-sharing prevista da TikTok, ossia la creazione di piccole clip video-musicali di brevissima durata (dai quindici secondi al minuto circa) provviste solo di un titolo simbolico e di un variabile numero di hashtag. Nella fattispecie, i TikTok relativi alla Shoah sono ritracciabili nell'applicazione attraverso due tag principali: il primo è appunto quello che indica la designazione del video come \#POV - acronimo di point of view - che cioè contraddistingue quella clip in cui l'utente dichiara di assumere una prospettiva diversa dalla propria, come se recitasse; il secondo marchio virale è quello che risponde invece dichiaratamente all'hashtag \#holocaustchallenge, lanciato su TikTok in riferimento alla specifica creazione di video il cui soggetto inquadrato 'faccia finta' di essere una vittima (quindi si interpretano persone defunte) della Shoah.

Il challenge prevede la regia e il caricamento di materiale multimediale in cui la cosiddetta Generazione Z (quella dei nativi digitali cresciuti dopo l'avvento e la diffusione consolidata a livello globale della rete internet) si sfida in cerca di like e numero di visualizzazioni rispetto al tema dello sterminio inventando una narrazione libera (cioè senza limiti di contenuto previsti da alcuna policy), nella quale appunto essi stessi 'diventano' (\#POV) finti prigionieri morti in un campo di concentramento. Il ricorso ai più noti stereotipi del genere è molto diffuso, come sottolinea Sophia Ankel:

In the videos, creators appear to be wearing makeup that imitates burns or bruises while explaining how they died in Nazi-run death camps. Other versions show people acting out representations of the genocide of Jewish people during the World War II, in some cases using the background image of the Auschwitz concentration-camp network. Some creators don a yellow Star of David like the ones Jews were forced to wear or dress up in striped shirts, to mimic the concentration-camp uniforms worn by the prisoners of Hitler's Third Reich. ${ }^{2}$

L'insistenza macabra sulla sofferenza fisica subita sembra essere una delle costanti più evidenti di questi brevi video, assieme all'iconografia più nota sul tema: le scelte più costanti sono risultate essere i lividi sul corpo creati con il trucco, la faccia annerita per imitare il lavoro forzato, un fazzoletto annodato intorno al volto (per le ragazze), la camicia a righe bianca e nera che vuole alludere alla divisa dei detenuti nei campi nazisti.

2 S. Ankel, «TikTok creators are pretending to be Holocaust victims in heaven in a new trend dubbed 'trauma porn'», Insider, August 23, 2020, https://www.insider.com/tiktok-trend-shows-people-pretending-to-be-holocaust-victims-heaven-2020-8. 
Lo sfondo 'artificiale' per eccellenza di molti video è invece quello in cui compare in lontananza l'immagine, ormai canonizzata, della torre del campo di concentramento di Auschwitz, di solito in bianco e nero, con gli annessi binari del treno in primo piano. In termini di setting, tuttavia, i filtri e costumi applicati al personaggio nel video contrastano spesso con l'ambiente circostante: si scorge a volte l'armadio della camera da letto che spunta dietro la persona che recita, così come il tavolo della cucina, o un altro ambiente domestico che decontestualizza profondamente l'esperienza multimediale della Shoah, etichettandola anzi come un prodotto 'amatoriale' che aspira ad esser valido in quanto tale, e non come testimonianza storica.

A parte questi escamotage, ciò che sorprende più di ogni altro dettaglio nei video TikTok taggati \#holocaustchallenge è l'estrema, disarmante e più o meno ingenua convinzione con cui il soggetto - regista e attore al tempo stesso - impersona la parte dell'ebreo assassinato: al di là del tipo di sentimento che il video punta a richiamare sul pubblico (si va dalla ricerca di una empatia e coinvolgimento drammatici, all'atto demenziale di fare ironia macabra sulla situazione del prigioniero), è evidente come il trauma e l'esperienza del dolore siano ciò che risulta davvero affascinante per i giovanissimi millennial, o almeno per quelli che hanno deciso di cimentarsi offrendo la propria rielaborazione storica dell'Olocausto. Questi remake della Shoah sono tuttavia stati percepiti subito come una rappresentazione irriverente, secondo alcuni addirittura pornografica, di una realtà terribile e totalmente estranea all'utente/consumatore, ma il fenomeno è rappresentativo di una tensione tra ciò che è necessario ritornare frequentemente a narrare e ciò che rende questa narrazione vacua e in fondo dannosa per il perpetuarsi della memoria.

La pagina web dell'Huffington Post Italia, dal momento che il fenomeno è scoppiato anche nel nostro Paese, riporta che:

Buone intenzioni a parte, è impossibile che brevi clip di TikTok possano dare spazio alla complessità di tali questioni e onorare adeguatamente le vittime. È come se ci fosse un gusto macabro della tragedia. La dottoranda Chloé Meley, in un articolo su Incite Journal, parla di «Trauma porn», dice Betti Guetta. Lo definisce come «il fascino perverso per la sfortuna di altre persone; un fenomeno che è diventato pervasivo in un'era digitale in cui il dolore è mercificato e le rappresentazioni sconvolgenti di esso private del loro impatto emotivo». È come se ci trovassimo in una società desensibilizzata alle storie tragiche. Ma che allo stesso tempo si nutre di tragedia come se fosse l'ultima avvincente serie Netflix. ${ }^{3}$ 
Il mensile americano Wired ha intervistato alcuni dei TikTokers coinvolti nell'esperimento chiedendo di argomentare le ragioni dietro la propria partecipazione al challenge. La maggior parte degli utenti ha dichiarato di aver sempre avuto le migliori intenzioni rispetto alla condivisione dei video da loro girati: in molti casi, i giovani intervistati hanno precisato di aver voluto in fondo educare e sensibilizzare la comunità degli utenti, soprattutto in quanto alcuni di loro condividano autentiche storie in famiglia circa l'esperienza della deportazione. Per il fatto che i loro nonni o bisnonni siano stati vittime della Shoah, alcuni TikTokers si sono dichiarati più 'legati' a questa storia e direttamente coinvolti di altri:

"I'm very motivated and captivated by the Holocaust and the history of World War II," [McKayla, 15, Florida] says. "I have ancestors who were in concentration camps, and have actually met a few survivors from Auschwitz camp. I wanted to spread awareness and share out to everyone the reality behind the camps by sharing my Jewish grandmother's story". ${ }^{4}$

Il portare testimonianza di una tragedia storica attraverso un atto di finzione multimediale, per lo più caratterizzato da caducità e brevità e realizzato da utenti giovani e inesperti, ha tuttavia dei rischi evidenti che sono subito sembrati chiari alla maggior parte delle persone entrate a contatto con questa vicenda:

This isn't the first time that 'raising awareness' or claiming to be producing educational content has been used to justify potentially disturbing or violent content that may be upsetting to some users. A similar trend on the topic of domestic abuse, where creators pretended to be battered women or abusive boyfriends, was reported on in April of this year, with identical reasoning behind their creation. But TikTok's short clips will always fail to give space to the complexity of such issues, or honour the victims adequately. ${ }^{5}$

Dunque quello dell'Holocaust challenge non è un caso isolato, ma una pratica di mediazione e produzione culturale che sta diventando il canale preferenziale da parte delle nuove generazioni per fare denuncia e per sensibilizzare (nei casi migliori) la comunità della rete globale verso situazioni di ingiustizia o disuguaglianza sociale, nel cui contesto si inserisce l'attenzione per i temi del genocidio nazista. Il

4 N. Froio, «We asked TikTokers why they're pretending to be Holocaust victims», Wired, August 21, 2020, https://www.wired.co.uk/article/tiktok-holocaust-pov.

5 N. Froio, «We asked TikTokers why they're pretending to be Holocaust victims», Wired, August 21, 2020, https://www.wired.co.uk/article/tiktok-holocaust-pov. 
modo dei ragazzi di narrare il trauma (presente, passato, individuale o collettivo che sia) è molto lontano non solo da qualsiasi testimonianza storica o resoconto diretto della Shoah, ma anche dalle più famose rielaborazioni letterarie e cinematografiche legate all'immaginario comune della deportazione nazista, che hanno una diversa ambizione artistica e consapevolezza teorica. Tuttavia la presenza e la diffusione di queste forme di narrazione restituiscono la percezione dell'attrattività forse anche inconscia di queste storie, di un Fascinating Fascism per dirla con Susan Sontag ([1974] 1982), che è più che mai pericolosamente vitale.

Un altro significativo esempio si può trovare su Instagram, dove il profilo denominato @eva.stories è in realtà un utente fittizio sul quale si trova il resoconto 'figurato' della vita di una ragazzina di nome Eva al tempo dell'Olocausto. Il settimanale britannico The Guardian descrive l'account Eva.Stories come:

A high-budget visual depiction of the diary of Eva Heyman - a 13-year-old Hungarian who chronicled the 1944 German invasion of Hungary - but features hashtags, internet lingo, and emojis used by a 21 st century-teenager. ${ }^{6}$

In sintesi, l'operazione della pagina Instagram è qualitativamente diversa dalla competizione virtuale di TikTok, in quanto la ricostruzione storica di Eva aspira ad essere rigorosa: la peculiarità (o il problema) è che lo fa simulando una serie di contenuti e video (appunto le stories) che la bambina ungherese avrebbe condiviso se, negli anni Quaranta del secolo scorso, la piattaforma Instagram fosse esistita.

Vale la pena specificare che il progetto è stato ideato e distribuito nel 2019 dal magnate israeliano Mati Jochavi e sua figlia Maya, discendenti da una famiglia ebrea di vittime e sopravvissuti allo sterminio nazista. Come dichiarato quindi sulla bio del profilo Eva.Stories, il tentativo è quello di agire sui nuovi canali di comunicazione «in memory of the 6 million Jews murdered in the Holocaust». Ma a differenza di un prodotto narrativo tradizionale, in questo caso l'interazione col pubblico è maggiore, perché gli utenti possono commentare ma anche condividere e modificare i contenuti con un effetto pericoloso e incontrollabile.

Produced with a multi-million dollar budget, 400 staff and actors, and elaborate sets including tanks and trains carriages, the stream of dozens of mini-stories were aired throughout Israel's Holocaust Remembrance Day this week. "Hi, my name is Eva. This

6 O. Holmes, «Instagram Holocaust diary Eva. Stories sparks debate in Israel», The Guardian, May 8, 2019, https://www.theguardian.com/world/2019/may/08/instagram-holocaust-diary-evastories-sparks-debate-in-israel. 
is my page. Follow me," says a young actor playing Eva Heyman in a trailer, dressed in a 1940s-style blue suit jacket, and filming herself selfie-style. She talks of her school crush and ambition to become a famous news photographer. Another post adds emojis of rainbows and strawberries. The tone of the Instagram videos turn darker as the Nazis increasingly target Hungary's Jews, confiscating Eva's family's business, making them wear yellow stars, and forcing them into a ghetto, before deporting them to Auschwitz death camp. ${ }^{7}$

Lo storico Simon Levi Sullam ha giustamente evidenziato, in un'intervista per Joimag sul fenomeno dell'Instagrammer 'Eva', che la prospettiva individuale della narrazione postmemoriale e la pratica di immedesimazione rispetto al genocidio nazista stanno prendendo sempre più piede come canale di trasmissione della memoria della Shoah presso i contemporanei:

I musei della Shoah, a cominciare da quello di Washington, hanno utilizzato negli ultimi trent'anni proprio l'associazione dei singoli visitatori con il destino specie delle vittime, puntando all'identificazione con i destini personali come vie di accesso alla comprensione storica. Credo che questa sia una chiave possibile ma che allo stesso tempo occorra far comprendere il contesto in cui si svolsero gli eventi; provocare reazioni e riflessioni anche con altri mezzi ad esempio artistici; sollevare domande sui pericoli dell'intolleranza e del razzismo oggi. Tutto ciò non passa solo attraverso l'identificazione né può esaurirsi in una app. ${ }^{8}$

Il processo di manutenzione e diffusione della memoria storica del genocidio non sarà destinato ad esaurirsi in rete, ma sicuramente la testimonianza della Shoah sta passando anche per questa porta - quella dell'immedesimazione diretta nella vittima, che dunque fa leva sulle forme più ovvie ed ingenue di storytelling come il ricorso all'empatia del fruitore.

Eppure il seguito che riscuotono queste iniziative, anche presso i più giovani, è un indice di come la popolarità sia un elemento ineliminabile nel ragionamento su questi temi, e questa popolarità ha probabilmente una doppia matrice: una matrice etica che porta gli utenti più ingenui a credere che occuparsi di questi temi sia giusto a

7 O. Holmes, «Instagram Holocaust diary Eva. Stories sparks debate in Israel», The Guardian, May 8, 2019, https://www.theguardian.com/world/2019/may/08/instagram-holocaust-diary-evastories-sparks-debate-in-israel.

8 M. De Pas, S. Levi Sullam, S. Ferrari, G. Schwarz, «Eva Stories. La Shoah ai tempi di Instagram», Joimag, 15 maggio 2019, https://www.joimag.it/eva-stories-lashoah-ai-tempi-di-instagram/. 
prescindere, senza chiedersi come occuparsene; una matrice narratologica che rende queste storie inconsciamente attrattive e dunque facilmente fruibili dagli utenti. Non crediamo spetti a noi occuparci direttamente della prima questione che andrebbe declinata piuttosto in termini filosofici, ma questo saggio vuole soffermarsi sulla seconda questione per verificare, attraverso alcuni casi campione, come questo interesse di ordine narratologico possa essere ricondotto proprio alla dimensione di romance che queste storie assumono. L'incrociarsi di questi due aspetti, ovvero dei presupposti etici e dell'attrazione romanzesca, comporta una sorta di ambivalenza nell'atteggiamento dei fruitori verso questi temi, come dimostra anche la conclusione del challenge di TikTok.

La 'sfida' social con oggetto la Shoah ha infatti creato ben presto un forte sdegno fra gli utenti non solo di TikTok, ma anche degli altri popolari social network, in cui sono apparse numerose denunce e frustrazioni da parte degli iscritti. La vicenda è così passata all'attenzione della stampa, dei musei e della Commissione Europea: lo scorso 8 settembre, TikTok ha finalmente revocato l'hashtag \#holocaustchallenge e «firmato il Codice di Condotta ${ }^{9}$ per lottare contro le forme illegali di incitamento all'odio online della Commissione Europea, unendosi così a Facebook, Twitter, YouTube, Microsoft, Instagram e altri» ${ }^{10}$ nel prevenire forme di trauma exploitation da parte degli utenti registrati al social network.

\section{Romance}

Questi episodi recentissimi rappresentano il sintomo evidente dell'ambiguità e della difficoltà di trattare la memoria prima e la storia poi di un evento indicibile come quello della Shoah, non solo perché l'altezza della tragedia non permette trovare parole per raccontarla, ma anche perché - al contrario - la sua enormità la rende un bacino di storie che inevitabilmente attraggono narratori e lettori. Come ammette Don DeLillo in Rumore bianco o in Running Dog o la stessa Susan Sontag o, con un'attitudine più provocatoria, Massimiliano Parente, rivolgersi a temi riguardanti la barbarie nazista o anche solo nominare Adolf Hitler assicura l'attenzione del lettore. Questo libro si propone di analizzare perché le storie su questo tema

9 Cf. il public statement pubblicato sulla pagina italiana del social network, lo stesso giorno della firma del Codice di Condotta della Commissione UE da parte di TikTok (08-09-2020): https://newsroom.tiktok.com/it-it/tiktok-aderisce-al-codicedi-condotta-contro-lincitamento-allodio-online.

10 B. Guetta, M.H. Cambruzzi, «15 secondi dai campi di sterminio. I video dei giovanissimi su TikTok», Joimag, 14 settembre 2020, https://www.joimag.it/15-secondidai-campi-di-sterminio-su-tiktok/. 
abbiano un impatto così forte sul pubblico, ipotizzando che esistano altre ragioni oltre all'urgenza etica che ovviamente non si vuole qui negare né ridimensionare nella sua portata.

Utilizziamo la parola romance nella sua accezione più alta e nobile. Il termine identifica anche un genere e uno stile bassi, caratterizzato per lo più da un sentimentalismo facile o da un susseguirsi di episodi rocamboleschi: «Al limite - scrive Paolo Zanotti -, romance può indicare semplicemente una storia d'amore e quindi anche quelli che da noi si chiamano "romanzi rosa"» $(1998,7)$. Qui non si tratta esattamente di questo, ma di uno stile alto e di un modo più che un genere. Lo spiega ancora Paolo Zanotti: «Il romance è piuttosto quello che si può definire un modo, cioè una serie di costanti dell'immaginario che si possono riflettere in letteratura all'interno di opere appartenenti a generi diversi» (8).

Il tono romanzesco, espressione con la quale potremmo tradurre il termine romance, dunque può rinvenirsi in molti testi senza essere necessariamente totalmente contrapposto al novel. All'interno della stessa opera perciò, a qualsiasi genere appartenga (ammesso che si possa parlare ancora di generi), è possibile trovare tracce di entrambe le forme, anche senza che ci sia una chiara dominante dell'una o dell'altra. In questo saggio si analizzano per lo più romanzi storici o che, pur appartenendo ad altri generi, abbiano moventi ben radicati nella realtà storica, e dunque almeno apparentemente più legati alle modalità realistiche del novel, ma questo non esclude una forte presenza di romance, che è il punto più critico nell'affrontare un tema storico.

Secondo Guido Mazzoni, «nel primo Ottocento, il romanzo che verrà chiamato realistico rende narrabile la vita quotidiana soprattutto perché immette, nel mondo del novel, un elemento del romance» (2011, 284). Il romanzo realista quindi non esclude il romance, anzi deve il suo successo proprio a questo. Il romanzo sul nazismo (ma si vedrà che utilizzare il singolare e fare un discorso generico conduce a molte approssimazioni) fa leva sulla stessa commistione di toni per affrontare il racconto di un evento reale, ma parte da presupposti pressoché opposti, ovvero la stupefacente e tragica enormità di quello che racconta. Mentre quindi la vita quotidiana del romanzo realista doveva essere resa narrabile attraverso l'immissione di elementi romance, in questo caso il romance è insito alle storie che vengono raccontate ma questo, come si vedrà, le rende, nella percezione del lettore, meno realistiche e dunque il romance fa un cattivo servizio alla memoria storica.

Il precedente più chiaro per comprendere quest'ultimo passaggio è quello del western. Basate sul più grande genocidio della storia umana, ovvero quello dei nativi americani, queste storie sono diventate divertenti e appassionanti narrazioni di genere, che hanno fatto perdere del tutto il senso della realtà dalla quale prendono le mosse. Il confronto tra indiani e cowboy è diventato un gioco e i film o i libri che ne parlano quasi mai alludono alla tragedia da cui tut- 
to parte. Lo spettatore percepisce come buoni gli autori del genocidio e come cattivi le vittime, con uno spaventoso equivoco che certamente non fa bene alla memoria.

Ovviamente, nel caso delle storie sulla Shoah non siamo giunti a questo livello, ma il precedente del western fa capire quanto il conflitto tra romance e realtà storica possa essere importante per comprendere il ruolo di queste narrazioni nella diffusione corretta della memoria. Si pensi a un caso come quello di Se non ora, quando? di Primo Levi: da una parte l'autore si premura di segnalare nella nota che conclude il romanzo che «i fatti che ho descritti sono realmente avvenuti, anche se non sempre nei luoghi e nei tempi che ho loro assegnati» ([1982] 2016, 673), dall'altra non esita, in conversazione con Philip Roth (Levi [1997] 2018, 635-46), a definire il testo proprio un western. È chiaro che per Levi non c'è nessuna ambiguità etica e il ruolo dei buoni non può che essere incarnato dai protagonisti della resistenza ebraica, ma è evidente comunque la necessità di riflettere sull'ambiguità tra la testimonianza storica (in questo caso indiretta ma documentatissima) e il racconto appassionante. Tra romance e Shoah.

\section{Struttura}

Questo libro è idealmente divisibile in due parti, la prima affronta il fenomeno da un punto di vista diacronico, la seconda - pur non trascurando le opportune differenziazioni tra le diverse epoche storicoculturali - da un punto di vista per lo più sincronico.

Nel primo capitolo, «La Shoah come oggetto culturale», si analizza la ricezione dell'evento storico nei decenni successivi, con particolare riguardo alla situazione negli Stati Uniti e in Europa. È un necessario discorso preliminare, perché molti termini, ma anche molte certezze, che oggi diamo per scontate, sono penetrati gradualmente e in tempi diversi nelle due realtà geografiche. Questo ovviamente ha condizionato la scrittura e la ricezione delle opere narrative, e viceversa queste ultime hanno condizionato la ricezione del fenomeno, soprattutto nei casi più popolari come il film Schindler's List di Steven Spielberg che certamente ha avuto un ruolo di spicco nella diffusione della coscienza della tragedia storica per la cultura di massa. La Shoah è diventata nel tempo un oggetto culturale, diffusissimo come abbiamo visto anche nei social network, con i conseguenti inevitabili rischi che sono stati efficacemente racchiusi nella definizione Pop Shoah (Recchia Luciani, Vercelli 2016).

Nel secondo capitolo, «Postmoderno e Shoah», si affrontano invece le narrazioni letterarie e cinematografiche mettendo in relazione il contesto culturale con la ricezione della Shoah, soprattutto per quanto riguarda il diverso rapporto con la realtà (e quindi anche col romance) del postmoderno e dell'età che ne è seguita. Le esplosioni 
narrative degli anni del postmoderno hanno per lo più ridimensionato l'importanza della fedeltà alla Storia, mentre negli stessi anni la storiografia si impegnava a comprendere quello che era davvero successo in quel periodo. Tutto questo ha portato attriti e contraddizioni - da Pynchon a Pasolini, da Dyck a Roth - che vanno indagate. Così come contraddittorio e problematico appare il rapporto con la realtà che si fa urgenza e rigore negli anni Duemila come emerge dal caso di HHhH di Laurent Binet (2010).

Altro testo critico e ambizioso, dalla grande potenza narrativa ma anche basato su una singolare consapevolezza teorica, è Europe Central di William T. Vollmann a cui viene dedicato il primo dei due casi di studio inclusi in questo libro. Questo testo rappresenta infatti la summa di tutti i problemi teorici aperti nei primi due capitoli, dalla ricezione della Shoah all'uscita dal postmoderno, dal rapporto con la realtà alla difficoltà della definizione di genere.

Il capitolo quarto dà l'avvio teorico a quello che abbiamo detto essere uno studio sincronico, stabilendo le coordinate del rapporto tra «Romance e Shoah». A partire soprattutto dalla teorizzazione di Frye si analizza come alcuni punti fermi del tono romanzesco siano insiti nelle narrazioni concentrazionarie e quindi necessariamente l'ambiguità di cui si è detto tra storia e storytelling pervada tutti i racconti sul tema, da quelli più vicini alla testimonianza a quelli segnati palesemente dall'impostura, come nel caso di Enric Marco raccontato da Cercas.

Non poteva mancare, in questo contesto, un approfondimento su Primo Levi al quale è dedicato il quinto capitolo. Con la consueta sensibilità che lo caratterizza, l'autore torinese comprende quanto sia rischioso finire nei territori del romance. Lo si è già accennato per Se non ora, quando?, ma anche nella Tregua, testo di testimonianza intriso di tono romanzesco, Levi cerca di esorcizzare questi aspetti disattivando completamente il lieto fine, secondo una pratica che segue anche nel romanzo del 1982. Si vedrà anche come lo scrittore ridefinisca una lingua per dire l'indicibile rappresentando un precedente per tutti i narratori che vengono dopo di lui.

Infine, si affronta un ultimo caso di studio, quello di Jonathan Littell e del suo Le Benevole. Alla fine del suo Teoria del romanzo, Guido Mazzoni segnala il romanzo tra i pochi che nei decenni a cavallo del secolo possano aiutare a descrivere le planimetrie della letteratura. Riporta tra l'altro una recensione negativa (prendendone le opportune distanze) che afferma: «Littell sarà riuscito nel tour de force di scrivere, in un anacronismo formale delirante, un romanzo sulla Shoah come se scrivesse un secolo prima di questo evento, che ha però cambiato per sempre la faccia della letteratura» (Bourmeau in Mazzoni 2011, 363). Ė davvero così? In questo capitolo si cercherà di considerare invece il testo come tematicamente tipico di quella che è stata chiamata l'età dell'inesperienza. 


\section{La Shoah come oggetto culturale}

Sommario 1.1 Shoah: realtà storica, narrazione, o entrambe le cose? - 1.2 Un problema onomastico. - 1.3 La Shoah nell'era della comunicazione. - 1.4 La Shoah come fenomeno di mercato. - 1.5 Età della postmemoria. - 1.6 Il ruolo della vittima.

\subsection{Shoah: realtà storica, narrazione, o entrambe le cose?}

Dal punto di vista storico, la Shoah è considerata il più grande spartiacque dell'età contemporanea: una tragedia senza precedenti che ha rimesso in discussione le logiche e il pensiero occidentale dell'ultimo secolo, modificando l'approccio sociale, culturale, economico e politico dell'individuo in ogni campo d'indagine della realtà storica. Secondo una prospettiva strettamente narratologica, invece, la somma degli eventi che indichiamo con il termine Shoah presenta una forte potenzialità romanzesca, specialmente grazie alla presenza di un pattern di dinamiche e circostanze che sono state spesso etichettate come 'eroiche', in relazione ai sopravvissuti. La netta contrapposizione fra personaggi positivi e negativi, che sta alla base di ogni narrazione mitologica, biblica e favolosa nella tradizione occidentale, si ripresenta con insistenza e chiarezza espressiva nella dicotomica rivalità fra i personaggi protagonisti del filone narrativo dell'Olocausto - i prigionieri nei Lager opposti alle SS naziste, ad esempio.

In questo senso, molti dei riferimenti alla Shoah nella realtà contemporanea si manifestano per descrivere e rendere esplicite situazioni soprattutto caratterizzate da ingiustizie sociali, repressioni violente da parte di un governo estremista, massacri di popolazioni e mancanza di libertà di espressione. Dall'uso (o abuso) che si fa del paradigma-Olocausto nel nuovo Millennio può sembrare che la Storia si ripeta, anche se non è davvero così: la memoria documentata 
dello sterminio degli ebrei è diventata parte inconscia di una collettività che ha smesso di percepire l'unicità storica di tale massacro, guardando alla lezione di cui è erede con toni sempre drammatici, ma inevitabilmente più distaccati. Quando è cominciato dunque questo processo di normalizzazione della Shoah che ha portato la sensibilità collettiva a riproporre le dinamiche di narrazione dello sterminio all'interno di contesti culturali, storici e comunicativi completamente scissi dal genocidio nazista?

Per capire come sia scattato un tale meccanismo di spostamento e riadattamento semantico rispetto a questa specifica fattualità storica è opportuno considerare alcune variabili fondamentali. Si prenda anzitutto il linguaggio: sebbene la terminologia impiegata negli Holocaust Studies e la semantica con la quale si ragiona di Shoah e nazismo siano un fatto linguistico relativamente recente (istituitosi dagli anni Sessanta in poi), l'uso di quello che potremmo definire il 'lessico concentrazionario', ovvero il vocabolario più immediato per far riferimento all'esperienza della deportazione e dello sterminio degli ebrei d'Europa, si è esteso in maniera capillare nei contesti sociale e comunicativo odierni, entro e fuori i confini dei Paesi in cui si è consumata, effettivamente, l'uccisione di massa degli ebrei e di altre minoranze europee per mano nazista.

Osservando nuovamente il fenomeno da un punto di vista narratologico, ci si accorge di alcune caratteristiche intrinseche e ben definite, proprie del mito della Shoah, riproducibili dai prodotti culturali nella società moderna: meccanismi narrativi tipici del genere romanzesco, come ad esempio la forte carica emotiva che si instaura fra lettore e personaggio nel genere tragico della narrazione (il pathos della tradizione greco-occidentale), ${ }^{1}$ la netta linea di demarcazione presente fra le categorie di 'bene e male', 'giusto e sbagliato' all'interno del suo bacino di significati antropologici ed etici e, soprattutto, un forte antagonismo generato dalla casistica dei suoi attori principali (i ‘buoni contro i cattivi'), ${ }^{2}$ hanno contribuito a rendere la Shoah un racconto fruibile e replicabile anche al di fuori della sua epoca storica. I caratteri di irripetibilità e indicibilità del genocidio nazista sono paradossalmente venuti meno per trasformare la Shoah sempre di più, invece, in un tag polivalente, applicabile a circostanze, usi e costumi del tempo presente in svariate forme e dimensioni narrative.

1 Illustrando i modi di invenzione del genere tragico nel celebre saggio Anatomia della critica, Northrop Frye insiste sulla potenza del pathos come caratteristica fondamentale della tragedia basso-mimetica nel creare una forte connessione fra il testo e il lettore: «Il pathos presenta l'eroe isolato da una forma di debolezza che fa appello alla nostra simpatia poiché è al nostro stesso livello di esperienza [...]. Il pathos, inoltre, contrariamente alla tragedia alto-mimetica, è accentuato dall'incapacità di esprimersi della vittima [...]. L'idea fondamentale del pathos è l'esclusione di un individuo del nostro stesso livello da un gruppo sociale al quale egli cerca di appartenere» (Frye 1969, 52-3)

2 Vedi il capitolo «Postmoderno e Shoah» in questo volume. 
Per poter ragionare in modo consapevole e responsabile su tali prodotti culturali, nati da rielaborazioni di specifici avvenimenti storici, è necessario anteporre una differenza concettuale, evidenziando come la progressiva estensione dell'area di riferimento dell'Olocausto per descrivere alcuni fatti recenti sia, appunto, un fenomeno soggetto a modifiche e riscritture continue. Lo scopo di questo contributo diventa allora quello di considerare le principali evoluzioni del paradigma Shoah al di fuori del proprio contesto di riferimento, osservandone le conseguenze e declinazioni più recenti in chiave diacronica: per comprendere le forme artistiche legate al mito della Shoah nel presente, insomma, partiremo anche noi dal passato.

Prima di avviare quindi l'indagine sui testi e modelli di riferimento rispetto agli usi della Shoah in modo finzionale, è necessario interrogarsi su quali siano stati i toni con cui si è inizialmente parlato di Auschwitz dopo il 1945, in Europa e nel resto del mondo, osservando se questi siano gli stessi con cui si rivolge ora attenzione ai medesimi argomenti. Viceversa, qualora sia subentrata una variazione nel tramandare il ricordo dell'esperienza concentrazionaria in tempi recenti, in che modo il medium attraverso cui si esprime e si declina la memoria collettiva della Shoah oggi risulta diverso da quelli del secolo scorso? L'intero processo costitutivo delle memorie storiche rispetto al genocidio ebraico - e la fatica che queste hanno fatto per poter esser universalmente riconosciute come parte integrante della coscienza identitaria dell'individuo moderno - sono fattori la cui genesi è opportuno osservare da un punto di vista prospettico, piuttosto che nel solo risultato ed eco che questi hanno nel nostro presente storico.

Nelle pagine seguenti si proverà dunque a ragionare sui diversi aspetti e sulle conseguenti implicazioni di ciò che effettivamente diciamo quando scegliamo di scrivere, discutere, o rappresentare in varie forme artistico-letterarie l'universo narrativo comunemente identificato sotto il termine Shoah: ci si focalizzerà prima sulla variabile linguistica della terminologia di riferimento base (§ 1.2), per poi riflettere sulle differenze di contesto storico, di pubblico e di sensibilità culturale (§ 1.3) che hanno permesso e autorizzato la rielaborazione dei valori e degli stilemi presenti nel mito della Shoah nella dimensione contemporanea (§§ 1.4 e 1.5). Infine, il saggio si chiuderà con una breve valutazione socio-antropologica della figura cardine dell'eroe, a livello narratologico, ripensata in funzione del ruolo della vittima nel presente contesto di riferimento (§ 1.6): questa valutazione finale vuole fornire un'ulteriore prospettiva d'indagine al lettore, legando la conformazione sociale dell'individuo moderno alle narrative dei prodotti culturali esaminati nel corso di questo contributo. 


\subsection{Un problema onomastico}

Nell'immediato dopoguerra è stato complicato trovare un canale linguistico adatto a denominare e interpretare - dunque storicizzare - l'eredità sociale, psicologica e antropologica del conflitto bellico appena conclusosi. Come nel caso di ogni novità storica con valore di 'spartiacque' fra un'epoca e l'altra, anche per il genocidio degli ebrei è stato necessario coniare un vocabolario tecnico che potesse soddisfare il bisogno di tramandare l'evento in sé, elaborando così il significato che ad esso era legato. Non solo: si tenga presente che l'atto stesso di identificare e descrivere verbalmente un avvenimento necessita, nella norma, di un lessico che sia da un lato specifico e puntuale, ma che dall'altro sia anche variegato e declinabile (cioè flessibile a livello grammaticale) per denominare il più correttamente possibile l'oggetto, il tempo e le motivazioni connesse a quanto descritto.

Sin dal 1945, la scelta relativa ai termini con i quali denominare lo sterminio degli ebrei ha costituito un tema ostico specialmente per il fatto che, fra le definizioni proposte, nessuna sembrava semanticamente appropriata e concettualmente 'completa': un sostantivo adatto ad esprimere un determinato aspetto della persecuzione razziale da parte nazista - come ad esempio la famosa formula «crimini contro l'umanità» coniata durante i processi di Norimberga - spesso finiva per omettere altri caratteri ugualmente significativi del medesimo momento storico (l'omicidio di un popolo), con il danno di relegare certi aspetti dello sterminio a dettagli irrimediabilmente latenti per le generazioni future, le quali andavano invece sensibilizzate e istruite rispetto ai fatti di Auschwitz.

In generale, la fine della Guerra ha coinciso con un momento di confusione sociale e strutturale diffusa, che ha interessato tutte le popolazioni coinvolte direttamente e indirettamente nel Secondo conflitto mondiale. In quei primissimi anni, è stato quindi difficile capire quale fosse il modo più adatto per trasmettere e conservare la memoria storica di un avvenimento che è tutt'ora considerato un caso singolare nella storia dell'umanità. La Shoah ha infatti provocato la necessità di una riformulazione e rivalutazione delle dinamiche storiche fino ad allora accadute in virtù del suo singolare statuto di violenza ed esasperazione dell'intolleranza di un popolo verso un altro. Sebbene tale necessità sia stata sentita a livello universale, è opportuno tener presente che vi sono state sostanziali differenze in merito alla percezione della fine del conflitto nei singoli contesti nazionali: il modo cioè in cui si è reagito all'eredità del disastro bellico appena conclusosi è cambiato da Paese a Paese, creando piccole differenze nel modo di parlare e raccontare dei crimini nazisti alla fine degli anni Quaranta. 
Raul Hilberg, ${ }^{3}$ il cui resoconto sulla questione ebraica è tutt'oggi considerato come il più autorevole e documentato fra i tanti a disposizione, non utilizza mai - né nel titolo né nelle sue quasi millequattrocento pagine di testo - alcuna delle definizioni più in voga fra gli storici e i sociologi moderni per parlare del massacro operato dai nazisti verso il popolo ebraico; quando lo fa, è per rendere conto di dinamiche e iniziative specifiche rispetto all'atteggiamento di un certo Paese o gruppo sociale nei confronti dell'elaborazione della memoria storica: in riferimento agli Stati Uniti, ad esempio, Hilberg riporta che «con un ritardo di venti anni sugli avvenimenti, l'annientamento degli Ebrei ebbe un nome: l'Olocausto» $(1995,1198)$; in altre nazioni, tuttavia, questa distanza cronologica di vent’anni è aumentata o diminuita a seconda dello specifico processo costitutivo dell'elaborazione dello sterminio in funzione di un determinato contesto socio-culturale.

La specie umana di Robert Antelme, ${ }^{4}$ ovvero uno dei testi fondamentali della letteratura sulla deportazione, viene pubblicato in Francia già nel 1947 (quindi con largo anticipo rispetto ai prodotti narrativi e cinematografici sulla Shoah distribuiti in America a partire dagli anni Sessanta), ma in questo libro si propone un'immagine di prigioniero molto diversa dalla tipologia di vittima che, più avanti, sarebbe stata canonizzata dalla letteratura sui campi di concentramento. Il deportato di Antelme è un personaggio anzitutto politico, che vede nel Lager il prodotto finale dell'incongruenza generata dalla società di massa: è una «nuova figura sociale e ontologica» (Scarpa 2010, 180), lontana sia dalla vittoriosa immagine dell'eroe di guerra ottocentesca (Les Misérables) sia dal dimesso e supplichevole ritratto dei superstiti dietro il filo spinato nei Lager, fotografati dalle truppe alleate nel momento della liberazione dai campi di concentramento.

L'unico fattore che emerge, trasversalmente ma in maniera uniforme, in ciascuna realtà nazionale nel primissimo dopoguerra è costituito da quella che potremmo definire come un'iniziale 'sospensione linguistica' del principio onomastico: ovvero in tutti gli stati occidentali si è registrata una preponderante fase di paralisi morfologica rispetto alla realtà da descrivere, dalla quale la comunità scientifica,

3 Cf. Hilberg 1995, La distruzione degli Ebrei d'Europa. N.B.: L'edizione Einaudi è basata sulla revisione dell'edizione originale del 1961 da parte dell'autore, pubblicata per la prima negli Stati Uniti nel 1985 (cioè dieci anni prima di quella tradotta per Einaudi in italiano, che fu poi pubblicata in due volumi per la collana dei Tascabili).

4 La prima versione italiana de La specie umana di Robert Antelme è stata tradotta e distribuita da Einaudi nel 1954, quattro anni prima dell'edizione canonica di Se questo è un uomo di Primo Levi (1958). Ad oggi, questo scarto cronologico è tenuto in rilievo dalla critica ai fini di una migliore comprensione della politica editoriale del tempo, unita alla ricezione generale della tematica della deportazione, nel panorama italiano (cf. l'«Introduzione» al testo di Antelme (1997) a cura di Alberto Cavaglion). L'edizione in commercio dell'Espèce in Italia è basata sulla riedizione Gallimard dell'originale francese (1957). 
politica e accademica ha cercato di svincolarsi costituendo un vocabolario ufficiale per definire e condannare i crimini nazisti. La portata di significato delle atrocità commesse durante la Seconda guerra mondiale è stata quindi a lungo soggetta ad un emblematico stato di aporia lessicale congenita, scontrandosi con una reiterata inadeguatezza della casistica nominale a disposizione per le varie forme del linguaggio orale e scritto. Gli stessi nazisti operarono in gran parte un processo di rimozione linguistica per designare i crimini e le procedure di sterminio nei campi di concentramento (Klemperer [1947] 2000): nessuna prova esplicita delle violenze commesse nei confronti dei prigionieri nei campi è infatti emersa dalla lettura dei verbali recuperati dopo la sconfitta tedesca. ${ }^{5}$

Nel caso dell'Italia, la lezione lasciataci dallo scrittore e testimone Primo Levi opera su due livelli di ragionamento complementari per quanto riguarda il linguaggio: per elaborare il ricordo delle vittime e dei crimini perpetuati dai nazisti nei campi di concentramento, Levi ha dovuto scegliere arbitrariamente, da una parte, un lessico specifico per denominare i luoghi, i metodi e i protagonisti che hanno formato la realtà concentrazionaria da lui conosciuta, mentre dall'altra ha riflettuto proprio sulla natura del linguaggio in generale, del suo legame inscindibile dalla condizione umana relativamente allo stato di cattività in cui si trovavano i prigionieri. In quanto discrimine fra ciò che è bestiale e non, la 'Babele' linguistica presente nel Lager ha di conseguenza rappresentato, dal punto di vista dell'autore, un potente tratto di imbarbarimento e impoverimento intellettuale a cui le vittime sono state sottoposte. ${ }^{6}$ E come se questo effetto avesse avuto un'eco anche nella dimensione sociale post-bellica, generando una fase in cui non si sapeva come raccontare degli orrori perpetrati a danno di altri esseri umani.

Nonostante Levi sia oggi lo scrittore più citato nel nostro Paese durante le occasioni pubbliche e istituzionali in ricordo delle vittime di Auschwitz, negli anni Cinquanta del secolo scorso il suo messaggio fu pressoché ignorato dalla critica e dal pubblico italiani. Questo rife-

5 «Prima di tutto, la burocrazia ci teneva a nascondere le sue azioni. Intendeva dissimulare il processo di distruzione non solo a tutti gli elementi stranieri, ma anche alla censura che la sua coscienza avrebbe potuto esercitare» (Hilberg 1995, 1145). Cf. anche p. 1146 e ss. per l'analisi specifica dell'autore sui meccanismi di rimozione nazista rispetto alle proprie colpe.

6 Nel capitolo «Iniziazione» di Se questo è un uomo si trova una prima, importante riflessione in merito: «Capisco che mi si impone il silenzio, ma questa parola è per me nuova, e poiché non ne conosco il senso e le implicazioni, la mia inquietudine cresce. La confusione delle lingue è una componente fondamentale del modo di vivere quaggiù; si è circondati da una perpetua Babele, in cui tutti urlano ordini e minacce in lingue mai prima udite, e guai a chi non afferra al volo. Qui nessuno ha tempo, nessuno ha pazienza, nessuno ti dà ascolto; noi ultimi venuti ci raduniamo istintivamente negli angoli, contro i muri, come fanno le pecore, per sentirci le spalle materialmente coperte» (Levi [1958] 2016, 163). 
rimento cronologico è un dato di cui tener conto: Levi è stato infatti un testimone precoce, che ha deciso di trasmettere in forma scritta il resoconto della propria prigionia quando questa, a sua volta, costituiva un evento storico per il quale ancora non esisteva un vocabolario ufficiale, nel primo dopoguerra e durante il decennio successivo. Nel momento in cui Se questo è un uomo prende forma, cioè subito dopo il 1945, l'evento che viene oggi designato mediante il riferimento biblico Shoah - o con l'equivalente termine Olocausto, ${ }^{7}$ diffuso soprattutto nel mondo anglosassone - non era stato ancora concepito e declinato secondo una terminologia standard e di uso comune. Il titolo stesso del resoconto di Levi costituisce in italiano, di per sé, una domanda indiretta, quasi a prova dell'assenza di risposte nel definire la tragedia appena conclusasi: sul piano grammaticale, infatti, il volume intitolato Se questo è un uomo rappresenta un interrogativo implicito, espresso nella forma sintattica di un potenziale periodo ipotetico (potenziale perché incompleto, composto cioè solo dalla protasi), che suggerisce un tentativo altrettanto parziale da parte di Levi rispetto all'analisi della realtà concentrazionaria, dove non sembrano disponibili clausole linguisticamente valide per reggere l'assurdità dei crimini perpetrati ai prigionieri nei campi.

Nel 2001, la storica Anna-Vera Sullam Calimani ha pubblicato uno studio nel quale si analizzano le diverse opzioni terminologiche a disposizione per descrivere la Shoah. Nel saggio I nomi dello sterminio, il ragionamento di Sullam Calimani prende avvio esattamente dalla constatazione delle suddette aporie linguistiche di cui si accennava sopra, dovute alla difficoltà che 'esprimere l'inesprimibile' ha comportato, nell'immediato dopoguerra, per tutta la comunità scientifica mondiale: «La terminologia usata per indicare lo sterminio è [...] insieme il risultato e la testimonianza di un approccio che non si è mai potuto e non si può ancora svincolare da implicazioni emotive» (2001, 11). Tali implicazioni hanno influenzato sia i testimoni che gli studiosi nel formulare un vocabolario pragmatico e legalmente valido per definire e contraddistinguere, al tempo stesso, lo sterminio nazista da altri eventi storici:

7 Anche il termine olocausto, al di là della sua etimologia greca (cf. Treccani - Enciclopedia on line 2017, s.v. «olocausto», https://www.treccani.it/enciclopedia/olocausto), è in realtà connesso alla sfera biblica, in particolare rispetto alla pratica sacrificale davanti al dio degli ebrei. Essendo un lessema più generico di shoah, quest'ultimo viene tendenzialmente preferito a olocausto proprio perché scisso a livello semantico da ulteriori e pregressi significati (che per questo si trova spesso scritto con la maiuscola iniziale «Olocausto», per distinguerlo cioè dal sostantivo generico). Si riporta per correttezza la definizione integrale del termine shoah sul dizionario online dell'Enciclopedia Treccani: «termine ebraico ("tempesta devastante", dalla Bibbia, per es. Isaia 47, 11) col quale si suole indicare lo sterminio del popolo ebraico durante il Secondo conflitto mondiale; è vocabolo preferito a olocausto in quanto non richiama, come quest'ultimo, l'idea di un sacrificio inevitabile» (Treccani - Enciclopedia on line 2017, s.v. «shoah», https://www.treccani.it/enciclopedia/shoah/). 
I nomi dati allo sterminio sono delle metafore che dimostrano in quali archetipi e in quali paradigmi di altre epoche gli eventi di quegli anni furono inseriti dai primi testimoni o commentatori e, successivamente, come queste metafore abbiano dato vita ad altre metafore che hanno lentamente modificato la nostra percezione dello sterminio stesso. (Sullam Calimani 2001, 11; enfasi aggiunte)

Oggi, fra coloro che scrivono o leggono delle vicende riguardanti i campi di concentramento, non si percepisce più la difficoltà che comportò il bisogno di coniare un vero e proprio linguaggio dello sterminio da parte dei testimoni, dei leader politici, degli storici, dei sociologi e di molti altri studiosi: ${ }^{8}$ descrivere il processo di eliminazione fisica - quindi anche linguistica ${ }^{9}$ - di un altro gruppo sociale ha pertanto comportato il ripensamento delle modalità tramite cui ci si rivolgeva al passato, all'interno della tradizione occidentale. ${ }^{10} \mathrm{E}$ importante comprendere dunque che la Shoah, spesso utilizzata come metafora nel contesto comunicativo odierno in riferimento a moltissimi fatti di cronaca o d'attualità, ha preso per prima il suo nome (o i suoi nomi, come vedremo) da un atto di creazione linguistica metaforico appunto, non mimetico o precostituito.

Sullam Calimani ragiona su come il termine tecnico Endlösung ad esempio, elaborato dai nazisti per evitare di scrivere letteralmente 'sterminio' nei documenti ufficiali del Reich, sia giunto in un primo momento ad essere usato come sinonimo di genocidio ebraico. Calimani pone l'accento sul rapporto fra lingua tedesca e lingua dei prigionieri rispetto al meccanismo di decodificazione dell'esperienza concentrazionaria in sé, durante e subito dopo la Guerra. La studiosa nota giustamente come una vasta serie di tecnicismi (il cosiddetto

8 Contro un uso 'tecnicizzato' del linguaggio per descrivere lo sterminio ebraico si è scagliato Bruno Bettelheim, il quale nel suo contributo del «L'olocausto una generazione dopo», soprattutto in riferimento al termine olocausto, parla di «circonlocuzioni» attraverso cui l'umanità ha cercato di rimuovere psicologicamente gli eventi, mediante un meccanismo inconscio di negazione che prende avvio proprio dalla scelta dei nominativi per designare l'assassinio di sei milioni di ebrei: «L'uso di locuzioni tecniche o coniate appositamente in sostituzione di parole del nostro linguaggio comune costituisce uno dei più noti e più diffusi sistemi per distanziarsi, in quanto divide l'esperienza intellettiva da quella emotiva. Parlare di "olocausto" ci consente di padroneggiarlo intellettualmente laddove i fatti nudi e crudi, se fossero chiamati con il loro nome consueto, ci sopraffarebbero emotivamente» (Bettelheim 2005, 108).

9 A tal proposito, si suggerisce la lettura dell'analisi descritta da Primo Levi nel capitolo «Comunicare» de I sommersi e i salvati, in cui l'autore insiste e approfondisce il rapporto fra violenza e dimensione linguistica nella realtà concentrazionaria: «è ovvia l'osservazione che, là dove si fa violenza all'uomo, la si fa anche al linguaggio» (Levi [1986] 2016, 1205).

10 «Perché la parola Auschwitz non fosse più semplicemente la denominazione tedesca di una città e di un campo nazista in Polonia ma un'icona del XX secolo, è stato necessario un mutamento del nostro modo di guardare al passato, di riconoscerne le rotture, di coglierne il senso» (Traverso 2004, 227). 
'gergo del Lager') avesse avuto da un lato l'effetto di descrivere gli ordini dei soldati nazisti con toni eufemistici mentre, dall'altro, le stesse parole assumevano un carattere di 'incubo in codice' per i prigionieri: era il tedesco a costituire la lingua franca dei detenuti, non lo yiddish; ${ }^{11}$ ciascun prigioniero indirettamente verbalizzava la propria tragedia attraverso un vocabolario straniero e attenuato, periferico e non puntuale rispetto a ciò che stava realmente accadendo in quel preciso contesto. Il nominativo universale scelto per far riferimento alla distruzione degli ebrei d'Europa non poteva quindi esser un sostantivo tedesco, lo stesso utilizzato dai nazisti, soprattutto nel rispetto di tutti coloro che sono poi sopravvissuti alla medesima tragedia.

Il termine genocidio, un lemma che suona apparentemente meno marcato sul piano del significato, rappresenta invece una parola dalla genesi piuttosto controversa: calco dall'anglismo genocide, genocidio è un sostantivo coniato prima dei processi di Norimberga, che non viene tuttavia da subito utilizzato nei contesti ufficiali. In generale, l'atteggiamento scettico nei confronti di questo neologismo, bollato di pressapochismo e inesattezza da parte della critica e degli storici moderni, ne ha impedito a lungo l'utilizzo che oggi, invece, sembra tornato ad essere molto abbondante. ${ }^{12}$ Genocidio è un vocabolo che necessita di una specificazione attributiva per designare, nel dettaglio, la Shoah: occorre cioè parlare di genocidio nazista, ebraico o degli ebrei per non cadere in pressapochismi e imprecisioni sul piano storico, linguistico e socio-culturale. Il fatto che genocidio richieda l'utilizzo di un sintagma anziché di un solo termine può aver giustificato il suo minor impiego rispetto al lemma Shoah, o al gemello Olocausto, rispetto al presente contesto di riferimento.

Per quanto riguarda la voce nominale Lager, invece, diminutivo di Konzentrationslager, ${ }^{13}$ è essenziale notare come «il germanismo ebbe

11 «Ogni vittima capiva il linguaggio in codice adottato dai nazisti e gli eufemismi che indicavano la morte. Queste espressioni erano così diffuse nei campi di sterminio da venire adottate anche dai detenuti, soprattutto quando dovevano parlare delle uccisioni e delle punizioni, ma non solo. Essi avevano sviluppato un loro gergo utilizzando però parole tedesche, le uniche in grado di esprimere una realtà che nei loro idiomi di origine non aveva nome: in tal modo il linguaggio degli oppressori contaminava quello degli oppressi» (Sullam Calimani 2001, 64-5).

12 Il termine genocide ha una storia interessante: dal momento che si riscontra spesso un atteggiamento generalmente accusatorio nei confronti del lemma, se applicato al caso specifico della persecuzione nazista verso il popolo ebraico (genocidio vs cultoricidio), s'invita a rileggere la storia della parola e le origini della sua coniazione da parte del giurista polacco Raphael Lemkin in prospettiva storicistica. Cf. Schaller, Zimmerer 2009, in particolare il saggio di Dan Stone, «Raphael Lemkin on the Holocaust» (95-106). Circa l'utilizzo debito o improprio del termine, nonché in riferimento ai vari modelli di genocidio a livello teorico, cf. Boghos Levon Zekiyan, «Quale rapporto tra la definizione giuridica e la realtà storica dei genocidi?», in Bernardini et al. 2006, 183-206.

13 Di qui l'abbreviazione «KZ» in Levi, presente anche sotto la grafia «Kazett», come traslitterazione fonetica della pronuncia tedesca in italiano (cf. Levi [1958] 2016, 
fortuna solo in Italia: non compare infatti nei dizionari francesi [...] né in quelli inglesi che accolgono solo il suo noto omonimo, denominazione di una birra a leggera gradazione alcolica, la cui diffusione ne impedì, con ogni probabilità, l'ingresso del germanismo» (Sullam Calimani 2001, 40). Per capire il motivo del successo di questa definizione in Italia, si torni alla lezione di Primo Levi: nei suoi libri testimoniali, si osserva bene come il linguaggio dei sopravvissuti sia vicino - nella memoria e nella scrittura - all'esperienza in sé della deportazione e del lavoro forzato nei campi. ${ }^{14}$

Nella fattispecie, il termine con cui Primo Levi si riferisce più frequentemente nei suoi testi a quella che oggi chiamiamo 'Shoah' è proprio Lager, un sostantivo che ha assunto e conserva valore metonimico per indicare lo sterminio degli ebrei quasi solamente in Italia - e questo è un merito della precocità della testimonianza di Levi rispetto ad altri contributi storici e letterari (cf. Sullam Calimani 2001, 39-40). Primo Levi, sempre utilizzando la lettera maiuscola a inizio lemma, ne fa un uso densissimo a partire da Se questo è un uomo, senza spiegare in nessun luogo dell'opera cosa esattamente Lager stia a significare: non ne aveva evidentemente bisogno, o meglio non vi era possibilità di fraintendimento, ${ }^{15}$ data la natura del discorso, per le persone con cui l'autore ha condiviso la propria esperienza di prigionia, o per coloro a cui Levi ha raccontato le sue storie una volta rientrato in Italia. ${ }^{16}$

In ultimo, soffermiamoci sul toponimo Auschwitz: la località polacca del sito concentrazionario che registra oggi un'ampia corrispondenza nell'uso comune del linguaggio, è stata precocemente utilizzata nei primi scritti leviani per alludere alla totalità dell'esperienza

234). In un'intervista con Milvia Spadi del 1986, che compare sotto il titolo «Capire e far capire», l'autore ha affermato: «Però veramente, ritenevo scrivendo e ritengo tuttora, che questa istituzione totale, quella del KZ - perché dire "campo" è una cosa diversa, c'erano campi di lavoro, campi di prigionieri militari, che erano diversi - è un unicum nella storia dell'umanità e vale la pena di parlarne come un unicum, mai ripetuto finora» (Levi [1997] 2018, 791).

14 «Nel narrare le loro esperienze i primi testimoni usarono, anche nei titoli delle loro opere, espressioni quali: deportazione, deportati, campo, campo della morte e campo di concentramento o, in alternativa, i toponimi» (Sullam Calimani 2001, 36).

15 «Il termine [Lager] era penetrato, invece, in italiano fin dai primi decenni del secolo ed è stato accolto nell'Appendice di Migliorini nel 1942 nel significato primario di 'campo' («residenza volontaria od obbligata. I minatori italiani mandati in Germania per lavori minerari o metallurgici risiedevano in Lager»), ma anche come abbreviazione di Konzentrationslager, campo di concentramento. La precoce accoglienza del forestierismo favorì in parte la sua penetrazione, sebbene l'ampia diffusione negli anni '60 sia certamente attribuibile alla notorietà acquisita dalle opere di Levi». (Sullam Calimani 2001, 40).

16 Cf. l'episodio in cui ne La tregua, il narratore protagonista e il greco si rivolgono al prete della mensa di Cracovia, in cerca di notizie e indicazioni: «Dalla iniziale richiesta di informazioni ("Pater optime, ubi est mensa pauperorom?") venimmo confusamente a parlare di tutto, dell'essere io ebreo, del Lager ("castra”? Meglio Lager, purtroppo inteso da chiunque)» (Levi [1963] 2016, 339). 
della persecuzione e dello sterminio ebraico - quindi il termine si è svincolato molto presto dal puro riferimento geografico. Anche Sullam Calimani insiste su come «la scoperta di Auschwitz e l'assunzione di questo nome in funzione simbolica sono parallele alla presa di coscienza della singolarità dell'eccidio degli ebrei» $(2001,71)$. Tale presa di coscienza, o «riattivazione della memoria», come l'ha definita Traverso $(2004,232)$, si verifica in un secondo momento, ovvero alla fine degli anni Cinquanta, quando, non a caso, si registra un maggiore successo e interesse da parte del pubblico per le pubblicazioni delle memorie dei sopravvissuti, nel panorama editoriale occidentale.

In termini di apprezzamento da parte dei lettori, la promozione della vasta letteratura sui campi di concentramento nel mercato editoriale ha costituito un fenomeno divulgativo ancora più recente. La canonizzazione letteraria e il relativo valore documentale delle memorie dei sopravvissuti si sono progressivamente costituiti fino a essere riconosciuti da tutti i gruppi sociali nella tradizione culturale contemporanea: anche questo è stato un processo che, tuttavia, ha avuto un lungo ed intermittente sviluppo. All'alba della stipula dei trattati di Parigi, in Italia come nel resto d'Europa, era ancora pericoloso parlare della scomoda eredità lasciata dalla voragine nazista: è stato difficile capire in che modo rivolgersi al pubblico con fine educativo circa i fatti appena trascorsi - dei quali nessuno nell'immediato voleva sentir parlare, essendo il trauma della Guerra ancora vivo e recente nella memoria collettiva.

Sempre nello specifico caso dell'Italia, il messaggio da veicolare si rivolgeva ad una nazione lacerata su due fronti: quello intestino della lotta antifascista condotta dalla Resistenza partigiana, e quello della persecuzione razziale, frutto dell'alleanza fra Italia a Germania nella prima fase della guerra. Giustamente Alberto Cavaglion ha sostenuto che:

Ogni volta che parla della situazione venutasi a creare in Italia fra settembre 1938 e febbraio 1944 (cioè fra emanazione delle leggi sulla razza e la sua deportazione da Fossoli ad Auschwitz) Levi ci mette in guardia contro i rischi dell'anacronismo. La storia, come la vita, ha delle asimmetrie che in Italia diventano paradossi. $(2008,719)$

Il valore che pertanto la letteratura, i giornali, le riviste e la propaganda politica hanno dato alla liberazione del Regno d'Italia ad opera della Resistenza costituisce la prima chiave di lettura autentica dell'esperienza della Shoah nel panorama culturale italiano - si ricordi che lo stesso Levi viene catturato in quanto partigiano, ${ }^{17}$ non

17 Cf. Paolo Momigliano Levi, «L'esperienza della Resistenza nella vita e nell'opera di Primo Levi», in Gentili-Tedeschi et. al. 1999, 61-75. 
perché ebreo. ${ }^{18}$ Parallelamente, nel primo decennio del dopoguerra, la Shoah non sembra esser intesa come calamità fattuale di cui urge rendere un resoconto storico: anzi, non ha elementi di contatto con la Resistenza antifascista, in un certo senso, almeno secondo la percezione che se ne ha negli anni Quaranta, e per questo ha rischiato di essere a lungo subordinata alla testimonianza e 'santificazione' di quest'ultima.

La percezione sociale e popolare, in senso etimologico della parola, dei fatti di Auschwitz va quindi sempre letta attraverso la lente del contesto storico e culturale di riferimento. A livello sociale, c’è poi da tener conto anche di un altro distinguo importante: mentre la popolazione europea di civili tentava di archiviare un terribile passato, i superstiti allo sterminio e alla deportazione nazista hanno invece insistito per far presente alla collettività quali crimini fossero stati commessi nei campi di concentramento. Sebbene il dolore nel ricordare fosse traumatico, e per lungo tempo i racconti sui campi di concentramento non hanno incontrato il gusto del pubblico e quello degli editori, molti dei testimoni non volevano rinunciare a trasmettere il proprio resoconto in forma scritta, orale o in versi, per sensibilizzare la comunità sociale a cui avevano fatto ritorno.

A conferma di questa affermazione, sta il fatto che è stato possibile individuare, a posteriori, nella Shoah il più grave evento del XX secolo in quanto ha segnato la necessità di istituire una memoria di tipo collettivo $^{19}$ per quanto riguarda le vittime e i sopravvissuti alla tragedia, a livello europeo ed internazionale: questa operazione è stata concepibile non solo per via dell'orrore senza precedenti dello sterminio nazista, ma anche per le dimensioni e la portata che tale soluzione ha toccato durante la Guerra. Secondo lo studioso Robert Gordon, l'Olocausto si colloca proprio come evento costituente la necessità di una memory culture in epoca moderna, ${ }^{20}$ caratteristica peculiare e propria delle civiltà contemporanee degli ultimi settant'anni.

Per capire come tale memoria si sia evoluta, modificata e sviluppata nel corso del tempo, è quindi opportuno approfondire nel prossi-

18 Levi viene catturato il 13 dicembre del 1943 in quanto membro della Resistenza, ma verrà imprigionato e mandato nel campo di Fossoli poiché confessatosi ebreo durante gli interrogatori. Cf. Alberto Cavaglion $(2008,723)$ in merito alla vicenda dell'arresto narrata nel racconto «Oro» de Il sistema periodico.

19 «Over the past twenty years, memorializing the Nazi persecution and extermination of Jews and other minority groups has become in many countries a civic duty supported by governmental institutions. In parallel, historians have begun to interrogate the conditions that made possible the consolidation of so peculiar a cultural formation, namely a "collective memory"» (Baldini 2014).

20 «The Holocaust has come to settle at the center of a widespread "memory culture" which sets the highest cultural, but also moral or ideological, store by the elaboration of the past through the reclamation of lived lives in memorial narratives and representation» (Gordon 2006, 85). 
mo paragrafo le differenze sociali, culturali e di contesto in relazione alla portata storica di significati raccolti e trasmessi dal paradigma della Shoah.

\subsection{La Shoah nell'era della comunicazione}

Si è già accennato al rischio che, nella cultura occidentale, l'utilizzo di termini specifici come Auschwitz, Lager, Shoah, Olocausto, genocidio, fascismo, nazismo e razzismo si manifesti sempre più spesso indicando un orizzonte di senso generalizzato: il linguaggio dello sterminio è entrato agevolmente nello scambio comunicativo quotidiano, suggerendo un ampio ventaglio di significati traslabili da una situazione all'altra, senza che necessariamente vi sia un effettivo collegamento con il periodo storico dei crimini nazisti. Un esempio chiarificatore potrebbe essere costituito dal neologismo inglese grammar $\mathrm{Nazi}^{21}$ letteralmente 'nazista della grammatica', divenuto famoso nel linguaggio del web per definire l'atteggiamento di intransigenza degli utenti nei confronti di un uso improprio della lingua, sul piano ortografico e morfologico. ${ }^{22}$ Questa definizione dimostra bene come il processo di traslazione del contesto comunicativo rispetto a quei termini chiave sopra elencati sia un fenomeno ampio, e in costante aumento. I 'nomi dello sterminio' sono sempre più spesso impiegati per sottolineare l'urgenza di fatti o situazioni d'attualità, in senso metaforico e iperbolico, rispetto ad un passato di cui quasi più nessuno, oggi, è stato vittima o testimone diretto.

Nonostante questo dato rappresenti un fenomeno culturale a cui la posterità sembra far sempre meno caso, ${ }^{23}$ l'utilizzo spesso incon-

21 Macmillan Dictionary 2009-20, s.v. «grammar Nazi». https://www.macmillandictionary.com/dictionary/british/grammar-nazi. In italiano, il termine compare spesso nella grafia uniforme grammarnazi, senza esser tradotto, ma con medesimo significato e funzione dell'originale anglofono (cf. Gheno 2019, 24-5).

22 Per contro, il termine ha generato il suo contrario giocando sempre sull'analogia storica: l'opposto di grammar Nazi è diventato grammar Communist, definizione che indica coloro che scrivono senza dar peso all'errore ortografico o logico-sintattico (Urban Dictionary 1999-2020, s.v. «grammar Communist», https://www.urbandictionary.com/define.php?term=Grammar\%20Communist).

23 Il problema di utilizzare le vittime della Shoah come metro di paragone in contesti di cronaca, legati alla dimensione politica o alla sfera dell'attualità, è un trend attorno al quale si è già aperto il dibattito: al di là della legittimità o meno di applicare la 'categoria' olocausto fuori dal proprio scenario storico-sociale, questo fenomeno è sintomo evidente del 'potere iconico' che la modernità attribuisce sia alle vittime dello sterminio che al campo di concentramento quali simboli di degenero e ingiustizia. Come nota la studiosa e docente Hila Shachar, le vittime subiscono necessariamente un processo di 'disumanizzazione' per poter esser ridotte a medium, strumento di giudizio per la denuncia di altre atrocità storiche: «they are useful tools as points of reference 
dizionato della terminologia relativa al genocidio ebraico è constatabile in svariati contesti quotidiani, dal caso più triviale ${ }^{24}$ a quello più drammatico: ad esempio, il problema delle morti nel Mar Mediterraneo dei migranti in fuga dai cosiddetti «Lager libici» ${ }^{25}$ verso l'Europa, così come il caso dell'immigrazione clandestina dal Sud America agli Stati Uniti lungo il confine con il Messico, ${ }^{26}$ rappresentano due fatti di attualità in cui sia la stampa che la politica si sono servite della metafora-Olocausto in modo da far leva sull'urgenza di denuncia dello statuto emergenziale nel territorio, considerato anche il tasso elevato del numero di vittime coinvolte.

L'immediatezza con la quale ci si appella alla Shoah per suscitare nelle comunità un sentimento di urgenza e pericolo rispetto ad un determinato scenario politico-sociale ${ }^{27}$ può essere vista come una conseguenza diretta tanto dell'affermazione degli studi e delle conoscenze relative alla Shoah su scala mondiale, ${ }^{28}$ quanto come un risultato dell'industria culturale sviluppatasi intorno ad essa, responsabile di

and comparison in contemporary political debate. It turns Holocaust victims and survivors into concepts, decontexualised imagery and generalisations, and erases their individuality as human beings». È dunque presente una componente di astrattismo tale da rendere i morti nei Lager «appropriated as political metaphors» (H. Shachar, «The Holocaust is not your metaphor to use in modern political debates», The Guardian, January 27, 2014, https://www.theguardian.com/commentisfree/2014/jan/27/the-holocaust-is-not-your-metaphor-to-use-in-modern-political-debates). Per una lettura comparata, dialettica in prospettiva storica e culturale dell'Olocausto all'interno degli Genocide Studies, cf. Rosenbaum 1996.

24 Nello stato di Israele, l'Olocausto è diventato per altro oggetto di un beauty contest: l'istituzione del concorso «Miss Holocaust Survivor» prende avvio già nel $2012 \mathrm{e}$ l'iniziativa, nonostante le polemiche scatenate, elegge annualmente la sua miss fra un numero di candidate sopravvissute ai campi di concentramento sul criterio della 'storia più bella', e non in base all'aspetto fisico (cf. R. Gagnor, «Miss Holocaust Survivor», Il Post, October 28, 2018, https://www.ilpost.it/robertogagnor/2018/10/28/missholocaust-survivor/).

25 Cf. F. Mannocchi, «La costa dei lager: i centri di detenzione dei migranti in Libia, dove neanche l'Onu entra», L'Espresso, 8 settembre 2017, http://espresso. repubblica.it/internazionale/2017/09/08/news/migranti-la-costa-dei-lager-1.309011. A. Soldo, «I campi non sono finiti con i lager nazisti», Internazionale, 24 marzo 2015, https://www.internazionale.it/opinione/antonella-soldo/2015/03/24/centriaccoglienza-cie-immigrazione.

26 A. Pitzer, «'Some Suburb of Hell': America's New Concentration Camp System», The New York Review of Books, June 21, 2019, https://www. nybooks.com/daily/2019/06/21/ some-suburb-of-hell-americas-new-concentration-camp-system/.

27 Cf. «The Holocaust debate: from eyewitness account to trauma-drama», in Berberich 2018.

28 Oggi la Shoah rientra ufficialmente nel programma scolastico di moltissimi paesi nel mondo. Cf. l'intera sezione web «The Holocaust Explained», una pagina gestita dalla Wiener Library for the Study of the Holocaust and Genocide di Londra, in cui si propone un percorso didattico ampio, basato su documenti storiografici, per l'insegnamento della Shoah negli istituti d'istruzione di ogni livello (https://www.theholocaustexplained.org). 
avere indirettamente autorizzato anche un utilizzo allegorico della Shoah spesso in negativo, con evidenti banalizzazioni di carattere populistico e stereotipico rispetto ai suoi contenuti.

Un recente, significativo esempio di questo utilizzo 'ribaltato' della metafora-Olocausto nella cronaca italiana può esser visto nel riferimento alla liberazione degli ebrei durante la Seconda guerra mondiale in antitesi al rimpatrio della cooperante Silvia Romano: stando alle sue dichiarazioni, ${ }^{29}$ la giovane italiana tenuta in ostaggio in Africa per diciotto mesi da parte di un gruppo estremista legato al Al-Shabaab si sarebbe convertita all'Islam durante il sequestro, in maniera del tutto spontanea. Il paragone con Auschwitz è improvvisamente scattato dopo le immagini rilasciate dai media all'aeroporto di Roma Ciampino, in cui si vede la ragazza che, al suo rientro in patria, è vestita negli abiti tradizionali islamici. Nella violenta polemica ideologica nata sulla stampa e sui social network relativamente alla conversione religiosa della donna, alcuni esponenti politici e personalità pubbliche hanno accusato la giovane di ingratitudine e «tradimento», dichiarando che «è stato come vedere tornare un prigioniero dei campi di concentramento orgogliosamente vestito da nazista». ${ }^{30}$

Sulla piattaforma Facebook, un consigliere della regione Abruzzo ha ribadito il concetto scrivendo: «Avete mai sentito di qualche ebreo che liberato da un campo di concentramento si sia convertito al nazismo e sia tornato a casa in divisa delle SS?». ${ }^{31}$ Questo post ha contribuito ad aumentare la diatriba circa la scelta religiosa di Silvia Romano, generando una serie di condivisioni da parte degli utenti della rete che hanno fatto propria la metafora del deportato 'traditore' attraverso dei riferimenti storici scorretti e di cattivo gusto, alimentando così lo shitstorm ${ }^{32}$ a danno della ragazza.

29 La volontaria milanese ha dichiarato di essere diventata «spontaneamente» musulmana, e non per costrizione dei suoi sequestratori. Cf. "Silvia Romano è tornata in Italia», Il Sole 24 Ore, 10 maggio 2020, https://www.ilsole24ore.com/art/contesilvia-romano-e-stata-liberata-ADbcpWP.

30 Il tweet è stato pubblicato dal giornalista e direttore de Il Giornale Alessandro Sallusti (@alesallusti,Twitter, 10 maggio 2020,2:40 p.m., https://twitter.com/alesallusti/status/1259463370262097920). Cf. anche «Silvia Romano, Alessandro Sallusti: "E' stato come vedere un prigioniero che torna dal lager vestito da nazista"», $L i$ bero Quotidiano, 1 maggio 2020, https://www.liberoquotidiano.it/news/personaggi/22573052/silvia_romano_alessandro_sallusti_come_vedere_prigioniero_lager_vestito_nazista.html.

31 Simone Angelosanto, Facebook, 11 maggio 2020, 9:30 a.m., https://www.facebook.com/photo?fbid=10220259871440597\&set=a. 1397408188877 .

32 Il termine shitstorm è ufficialmente entrato a far parte del dizionario inglese e di altre lingue, come prestito, per definire appunto la gogna mediatica generata dal social media marketing rispetto alle polemiche generate sui social o in rete partendo, in genere, da un piccolo dettaglio o commento ad un determinato fatto che altera la narrativa del fatto stesso - da qui l'idea di tempesta (in inglese storm) del neologismo anglo- 
Al di là delle implicazioni politiche e dei giudizi morali che una tale affermazione può suscitare, in questa sede è interessante sottolineare che un paragone simile - quello fra Islam e nazismo - è oggi possibile proprio perché il genocidio ebraico funge da paradigma e bacino di significati oltre la propria dimensione storico-politica. Occorre, pertanto, filtrare i possibili paragoni sempre con la consapevolezza di una differenza ontologica alla base di ogni utilizzo traslato del lessico concentrazionario: gli episodi mediatici descritti infatti con la medesima enfasi linguistica della Shoah, relativa alla deportazione e uccisione degli ebrei, sono momenti storici completamente scissi dagli eventi dalla Seconda guerra mondiale. Sebbene questa sia una differenza sostanziale fra tempo passato e presente, si osservi come, sul piano linguistico, le differenze si stanno invece appianando: il caso di Silvia Romano, così come altri riferimenti alla Shoah totalmente decontestualizzati dall'originale situazione comunicativa, dimostrano quanto l'utilizzo del mito dell'Olocausto si sia profondamente acclimatato nella nostra sfera della comunicazione.

Oggi, la memoria storica del genocidio ebraico si riverbera nella quotidianità con una pericolosa e marcata efficacia, che quasi annulla le differenze temporali fra un evento e l'altro. Quando è iniziato allora, indicativamente, lo slittamento di significato veicolato dalla trasmissione della memoria storica della Shoah in direzione di una versione più mediatica e popolare (nel senso di fruibilità di massa) della stessa? L'acclimatamento dell'Olocausto ha generato una preponderante presenza di prodotti cinematografici, romanzi, rivisitazioni artistiche e monumenti che richiamano ad una versione già traslata e istituzionalizzata dell'esperienza concentrazionaria, ma questa alternazione a livello semantico, a sua volta, è stata originata da una fattualità storica precisa, che per prima ha rivoluzionato il modo di rappresentare lo sterminio presso la posterità: il processo Eichmann. Nel maggio del 1960, in piena Guerra Fredda, l'ex SS e funzionario nazista Adolf Eichmann viene arrestato dai servizi segreti israeliani a Buenos Aires, in Argentina: poi trasferito a Gerusalemme, Eichmann verrà processato per «crimini contro l'umanità» l'11 aprile dell'anno successivo.

Il processo ad Adolf Eichmann del 1961 è stato un evento che ha sconvolto e riassestato l'intera geometria concettuale del genocidio ebraico: soprattutto, più di ogni altra testimonianza che fino ad allora poteva aver avuto risonanza su larga scala, il processo Eichmann ha costituito un vero e proprio risveglio dell'interesse nei confronti dell'Olocausto da parte dell'opinione pubblica mondiale. La differenza sostanziale si colloca nel medium comunicativo: se il tribunale

fono (cf. Cambridge Dictionary 2020, s.v. «shitstorm», https://dictionary.cambridge.org/dictionary/english/shitstorm). 
di Norimberga aveva operato a porte chiuse nel condannare i propri imputati, il processo Eichmann viene invece distribuito come un vero e proprio evento mainstream in diretta televisiva da parte delle troupe americane (cf. Lipstadt 2014). Questa modalità di fruizione ha generato ripercussioni di tipo culturale, narrativo e mediatico importantissime per quanto riguarda la ricezione e la conoscenza dell'Olocausto da parte della popolazione mondiale.

Innanzitutto, la trasmissione in diretta del processo ha avuto il merito di forgiare una conoscenza di base più limpida circa la Endlösung e su quanto fosse effettivamente successo nei campi di concentramento nazisti. Questo ha chiarito pertanto gran parte dei pressapochismi che fino ad allora erano stati il fulcro della manipolazione e rielaborazione del concetto di nazismo, soprattutto in America ma anche in altri Paesi. In particolare, l'effetto prodotto negli USA ${ }^{33}$ è assai rilevante in questa sede di indagine dal momento che, nel prossimo paragrafo, noteremo come le prime e più importanti trasposizioni artistiche della Shoah nascono proprio negli Stati Uniti: con il processo e la condanna pubblica di Eichmann, per la prima volta il pubblico americano, ebreo e non, sente infatti parlare direttamente le vittime sopravvissute ai Lager nazisti davanti ad un tribunale in cui è presente l'emblema del nazista per eccellenza, il cattivo definito «banale» dalla Arendt che aveva visto in lui non un mostro, bensì un uomo terribilmente normale, eppure responsabile della morte di milioni di innocenti. ${ }^{34}$

Il 1961 è dunque l'anno del discrimine, ovvero il momento in cui si riporta l'attenzione all'Olocausto trattandolo come un'eredità storica scissa da nazisti o comunisti, separandolo anche dalla Guerra stessa: il trauma del genocidio ebraico riemerge in quanto vero e proprio assassinio di un popolo per colpa dell'odio antisemita e della repressione dei diritti umani civili da parte di un regime totalitario. Negli anni Sessanta, il ritorno (o sarebbe meglio dire l'avvento vero e proprio) dell'Olocausto nella cultura collettiva di massa, soprattutto americana, è decisivo per la pubblicazione, trasmissione e infine successo di tutti quei testi che difendono la memoria storica legata alla denuncia dei crimini nazisti: una tale evoluzione ha avuto origine a livello concettuale proprio con la cattura e la condanna di Eichmann di fronte al tribunale di Gerusalemme.

A livello socio-antropologico, il processo pubblico ad un criminale nazista ha posto l'umanità di fronte ad un nuovo tipo di responsabi-

33 «Negli Stati Uniti la storia occupò le prime pagine dei media. La CBS dichiarò che l'informazione aveva "elettrizzato il mondo... come se fosse stato trovato Hitler in persona”» (Lipstadt 2014, 21).

34 Eichmann si dichiarò sempre innocente e mai colpevole - Hannah Arendt lo paragona a Ponzio Pilato, in quanto «qualunque cosa facesse, a suo avviso la faceva come cittadino ligio alla legge» (Arendt 2001, 142). 
lità: vi era un ingombro morale di dimensioni nuove, una densità etica ineliminabile che, da questo punto in avanti, chiamava i superstiti diretti e indiretti a non poter evadere dall'urgenza di trasmettere quanto accaduto per prevenire il ripetersi della Storia, e punire coloro che ne erano stati i colpevoli. Enzo Traverso parla di «momento catartico di liberazione della parola» (2004, 232); la stessa formula «crimini contro l'umanità», impiegata nei documenti ufficiali a Norimberga, si dissolve spontaneamente per lasciar spazio a quei nomi e a quelle definizioni precise di cui si è parlato nel precedente paragrafo, e che tuttora costituiscono il vocabolario maggiormente in voga per indicare lo sterminio degli ebrei da parte della Germania nazista.

Circa l'uso quindi - e il conseguente abuso - di parole come Shoah, Olocausto, Soluzione finale e Auschwitz, occorre tenere a mente il fatto che questi termini costituiscano, in realtà, una coniazione linguistica a posteriori: una conseguenza verbale della prima, fondamentale rielaborazione commercializzata dell'Olocausto avvenuta negli anni Sessanta. La Shoah è stata infatti proposta (per non dire venduta), già nel 1961, secondo il format dello show televisivo da parte delle reti statunitensi: la più grande tragedia della storia umana, forte catalizzatore di empatia e compassione per le vittime e di orrore e disgusto verso il carnefice da parte del grande pubblico, è diventata ad un tratto uno spettacolo fruibile in diretta da Gerusalemme, seguito da tutto il mondo comodamente dal divano di casa.

\subsection{La Shoah come fenomeno di mercato}

Secondo una prospettiva diacronica, potremmo dire che il processo Eichmann costituisca l'antenato comune a moltissime delle rielaborazioni storiche e documentarie del genocidio nazista del secondo Novecento. Dopo un periodo di circa vent'anni in cui era stato ignorato, il concetto di Holocaust si trasforma rapidamente dal 1961 in avanti ${ }^{35}$ dal punto di vista socio-culturale e mediatico: con riferimento al panorama statunitense, che per primo ha intuito il potenziale del messaggio celato nell'esperienza concentrazionaria, è opportuno citare alcuni esempi significativi di prodotti culturali a carattere memoriale che, dopo il 1961, hanno contribuito nel plasmare e definire secondo parametri nuovi un atteggiamento narrativo e descrittivo della Shoah sempre più romanzato e dunque, spesso, lontano dalla realtà.

Rispetto al format della diretta Eichmann, la Shoah diventa nel 1978 protagonista della sceneggiatura di una fortunata miniserie televisiva dal titolo programmatico Holocaust, prodotta negli USA e di- 
stribuita a livello mondiale ${ }^{36}$ negli stessi anni. Sebbene precoce, $\mathrm{Ho}$ locaust non è stato il primo caso in cui l'universo nazista raggiunge visibilità mediatica. Prima di questo TV show, altre pellicole avevano già 'sfruttato' il potenziale della realtà concentrazionaria e della logica nazista creando lungometraggi che si sono contraddistinti grazie ad una carica narrativa nuova, in cui è ben evidente che il modo di parlare dei crimini nazisti stava subendo un potente rimodellamento romanzesco e satirico. Nel musical del 1968 The Producers (conosciuto in italiano con il titolo Per favore, non toccate le vecchiette), diretto e interpretato da Mel Brooks, ${ }^{37}$ un insieme quasi grottesco di tonalità sarcastiche e umoristiche rispetto alla rappresentazione dei nazisti nella trama sono sprigionate nell'esilarante messa in scena dello spettacolo Springtime for Hitler. ${ }^{38}$ Anche se giocata sul contrasto fra l'indignazione del pubblico e la felicità dei protagonisti (che di proposito hanno scelto di rappresentare sul palco una pièce teatrale impopolare e di cattivo gusto), in complesso la scena mette ben in mostra il livello altro su cui si muove la satira del nazismo nei confronti del pubblico.

In altri casi di film prodotti negli stessi anni, il ruolo dei nazisti è presente anche solamente in sottofondo - cioè l'esercito tedesco ricopre allegoricamente la parte del villain da sconfiggere come ultima prova per lo scioglimento della vicenda, la quale spesso non riguarda in realtà nemmeno la Seconda guerra mondiale (almeno nei temi principali). Si pensi al musical The Sound of Music (Tutti insieme appassionatamente) di Robert Wise (1965) ${ }^{39} 0$ al film Bedknobs and Broomsticks (Pomi d'ottone e manici di scopa), prodotto da Walt Disney nel 1971: ${ }^{40}$ nonostante in entrambi i film la vicenda sia ambientata in Europa (in Austria e in Inghilterra), il fatto che le pellicole siano state realizzate, prodotte e distribuite negli Stati Uniti dimostra quanto, già verso la fine degli anni Sessanta, l'immaginario nazista avesse colonizzato parte delle rappresentazioni artistiche nel mondo del cinema e della letteratura americana. ${ }^{41}$

36 Holocaust (1978). Dir. Marvin N. Chomsky. Titus Productions: NBC. La serie americana, composta di quattro puntate, ha vinto otto Emmy Awards.

37 The Producers [trad. it. Per favore, non toccate le vecchiette] (1968). Dir. Mel Brooks. Embassy Pictures.

38 Cf. lo spezzone del film al link: https://www.youtube.com/watch?v=HPXHRX8Q2hs.

39 The Sound of Music [trad. it. Tutti insieme appassionatamente] (1965). Dir. Robert Wise. Twentieth Century Fox Film Corporation.

40 Bedknobs and Broomsticks [trad. it. Pomi d'ottone e manici di scopa] (1971). Dir. Robert Stevenson. Walt Disney Studios Motion Picture.

41 Un discorso a parte (che non tratteremo in questa sede ma che è giusto menzionare) riguarda il mondo del fumetto, dove sono stati creati eroi ed anti-eroi ispirati dall'immaginario nazista, ad esempio Capitan Nazi o lo stesso Capitan America, nato nel 1941 come simbolo di una propaganda liberale della democrazia americana. 
Nel caso di Dr. Strangelove or: How I Learned to Stop Worrying and Love the Bomb, ${ }^{42}$ ad esempio, il personaggio del Dottor Stranamore, ex-nazista 'redento' che ora collabora con i servizi segreti americani, avvalora del tutto la tesi di quanto il processo di assimilazione dell'universo nazista si fosse ampiamente compiuto già nel 1964 - anno di uscita nelle sale della pellicola di Kubrick. La dinamica di sdrammatizzazione e semplificazione dell'universo nazista quale simbolo di oppressione e ingiustizia, da cui si è diramata e allargata la rete di significati legati alla sfera dell'Olocausto, ha radici dunque più profonde e lontane rispetto molte delle ultime rielaborazioni e drammatizzazioni della Shoah in tempi presenti. Gli esempi forniti non sono che un minimo campione tratto del consistente bagaglio narrativo e artistico delle declinazioni fantastiche, satiriche, distopiche e diacroniche formatesi dall'estensione del fenomeno-Shoah nell'industria culturale americana.

In un controverso studio degli anni Duemila, Norman Finkelstein ha riqualificato lo sterminio degli ebrei d'Europa da parte del Reich nazista esattamente al pari di un oggetto di produzione di massa. Nel suo saggio dal titolo provocatorio The Holocaust Industry, Finkelstein ha riflettuto su come sia stato possibile che la macchina consumistica americana abbia ingurgitato gli stilemi di una memoria storica che sembrava incorruttibile - in quanto tragica - sfruttandone proprio il carattere indiscusso di unicità della sua violenza e sofferenza per produrre capitale (cf. Finkelstein 2000, 43-8). Paradossalmente infatti l'Olocausto, in virtù del suo essere incomparabile, ha forgiato un mito da raggiungere, sviscerandosi dalla sua dimensione storica per appunto declinarsi in una rappresentazione ideologica molto diversa dalla reale realtà dei fatti, seguendo tempistiche alquanto precoci a livello di scarto cronologico fra la fine della guerra e le date di produzione delle pellicole citate poco fa. ${ }^{43}$

Nei precedenti paragrafi, abbiamo già in parte messo l'accento su come la Shoah sia oggi trattata non solo in termini di prodotto culturale finalizzato al consumo di massa - è possibile parlare di Shoah Show (cf. Recchia Luciani, Vercelli 2016) - ma anche come vera e propria chiave di lettura per decifrare alcuni aspetti della contemporaneità:

42 Dr. Strangelove or: How I Learned to Stop Worrying and Love the Bomb [trad. it. Il dottor Stranamore - Ovvero: come ho imparato a non preoccuparmi e ad amare la bomba] (1964). Dir. Stanley Kubrik. Columbia Pictures.

43 "In the pages that follows, I will argue that "The Holocaust" is an ideological representation of the Nazi holocaust» (Finkelstein 2000, 3). Nella sua trattazione, Finkelstein distingue anche a livello ortografico i due termini di analisi segnando con la maiuscola la continua rappresentazione (Holocaust) di quello che invece è solamente stato un evento storico (Nazi holocaust). 
un po' come in tutti i campi disciplinari, come e anche soprattutto nella stessa vita associata, il ricordo dello sterminio è divenuto, per molti, una sorta di elemento costitutivo della cittadinanza democratica, definendo la linea di divisione tra "civiltà" e barbarie. (Recchia Luciani, Vercelli 2016, 6)

Una delle conseguenze dirette, generatasi dall'onnipresenza del paradigma-Olocausto nella sfera del dibattito pubblico e della società dei consumi in età contemporanea, è stato l'aumento del livello di accessibilità che, proporzionatamente, ha accompagnato la fruizione della Shoah da una parte di un pubblico assai maggiore rispetto al secolo scorso.

I consumatori sono cioè aumentati in numero e competenze: non solo è incrementato il numero di persone interessate alla Shoah come evento storico, ma anche il valore commerciale che la redditizia 'industria dell'Olocausto' ha contribuito ad alimentare è cresciuto in termini di guadagno. Essendosi significativamente ampliato il bacino d'utenza tra gli anni Sessanta e Ottanta di coloro che potevano accedere ai media e ad un livello di educazione medio-alto, la consapevolezza riguardo al genocidio ebraico e al valore simbolico che esso ricopre si è pressoché espansa alla quasi-totalità della popolazione occidentale, svincolandosi dall'essere un fenomeno prettamente circoscritto alla sola orbita delle comunità ebraiche ${ }^{44} \mathrm{o}$ del sapere universitario.

A discapito infatti della qualità e del grado di approfondimento dei suoi contenuti, la quantità di persone che sanno o hanno sentito parlare dello sterminio nazista è in ogni epoca sempre maggiore: l'utilizzo frequente dei contenuti relativi al genocidio nazista nello scenario mondiale ha generato anche pratiche virtuose per la tutela del ricordo delle vittime di Auschwitz. Delineandosi sempre di più come una sorta di «mito commerciale» (Belli in Recchia Luciani, Vercelli $2016,17-29)$ la storia del genocidio ebraico è stata declinata in forme di consumo di massa che hanno però portato anche alla promozione di iniziative formative importanti, quali l'insegnamento della Shoah nelle scuole, la creazioni di siti museali e fondazioni per la ricerca degli studi sul genocidio di massa; per contro, tuttavia, o meglio in linea con l'interesse che la conoscenza dell'Olocausto ha riscosso in tempi recenti, si è assistito anche ad un mutamento di pertinenza della sfera di dominio della Shoah che ha toccato sia il campo delle arti visive e performative che quello delle discipline umanistiche - soprattutto per quanto riguarda la trasposizione finzionale dell'universo nazista in cinema e letteratura.

44 Sui modi in cui la consapevolezza della Shoah si sia poi corrotta e contaminata ad altri aspetti della società americana, cf. il contributo «The Assault on Holocaust Memory» di Alvin H. Rosenfeld (2002). 


\subsection{Età della postmemoria}

L'Olocausto è progressivamente diventato un oggetto simbolico altamente riproducibile dalle comunità umane proprio per la sua continua capacità di esser riattualizzato, a seconda della tipologia di rappresentazione scelta: si è visto come il primo 'utilizzo' mediatico della Shoah sia stato proprio una 'trasmissione' in diretta (il processo), disponibile nelle televisioni di tutto il mondo. Successivamente alla diretta Eichmann del 1961, abbiamo descritto come molti altri importanti prodotti di cultura massificata sono stati distribuiti presso il pubblico moderno fino a generare una vera e propria attività industriale della memoria, ovvero una meccanica e insistente produzione di forme culturali finalizzate alla trasmissione e narrazione della Shoah. Considerando il 1961 come anno della 'svolta', non è tuttavia possibile isolare completamente l'evento capostipite della trasformazione della Shoah dalla più ampia, complessa e intricata catena di eventi che, da quella data ad oggi, hanno modificato il tessuto sociale moderno. Molti altri fattori hanno infatti concorso ad alterare i toni e le pratiche culturali con cui è stata generata e trasmessa la memoria storica dello sterminio degli ebrei presso il pubblico contemporaneo - a definire quella che Annette Wieviorka ha chiamato «l'Ere du témoin», ossia propriamente 'l'era del testimone', nel suo omonimo saggio (1998).

Fra questi, un dato fondamentale da considerare nella presente sede d'indagine è costituito dall'avvento di una nuova conformazione sociale: una società formata da individui calati nella cosiddetta 'dimensione della postmemoria' in numero sempre maggiore. La definizione è della studiosa Marianne Hirsch, che nel 2012 ha parlato di «generation of postmemory» riferendosi alla conformazione demografica degli anni Duemila, composta sempre di più e quasi esclusivamente da individui che non hanno vissuto direttamente gli eventi di cui producono un ricordo per i propri contemporanei: in aggiunta, la matrice con cui tale ricordo è proposto sta diventando sempre meno di carattere storico, lasciando spazio a prodotti culturali di natura artistica, narrativa, o performativa. L'individuazione di un'età della postmemoria pone indirettamente l'accento sul distacco generazionale ${ }^{45}$ che, al momento, sussiste fra l'abbondanza di tutti i prodotti culturali incentrati sulla Shoah da una parte e, dall'altra, l'inevitabile scomparsa invece dei testimoni diretti di quel medesimo evento, che rimangono comunque, a tutti gli effetti, gli unici e i più autorevoli detentori della verità storica circa i fatti di cui s'intende ancora lasciare memoria ai posteri.

L'aspetto più interessante rispetto alle pratiche culturali di età post-memoriale è costituito però da un altro fenomeno, che di queste 
rappresenta un effetto collaterale immediato: in concomitanza con il progressivo venir meno dei testimoni diretti, si osserva infatti come, paradossalmente, le cerimonie in ricordo delle vittime del genocidio nazista, le iniziative culturali e museali, nonché i prodotti narrativi e cinematografici continuamente distribuiti sul mercato globalizzato sembrano, al contrario, dimostrare che la memoria della Shoah sia più viva che mai. La Shoah non ha dunque perso il suo valore archetipico, benché le generazioni successive a quella che ha partecipato alla guerra siano detentrici di un ricordo indotto, trasmesso appunto sempre meno da chi è stato testimone diretto di quegli stessi eventi, e tramandato invece da individui che, al pari del pubblico destinatario, hanno avuto una memoria altrettanto indiretta dell'esperienza concentrazionaria.

Questo distacco generazionale sempre più ampio fra l'individuo di oggi e l'evento storico di ieri ha creato una distanza che non è più solo temporale dunque, ma anche cognitiva e percettiva rispetto alle modalità di rielaborazione e racconto del trauma dello sterminio: se la memoria di un evento viene infatti prodotta da un individuo che non ha memoria 'reale' di quello stesso evento - essendo anche lui stesso un epigono rispetto al momento storico di cui vuol render conto - ecco che la tendenza a raccontare 'esagerando' quelle dinamiche narrative proprie degli agenti o del contesto in cui sono calati i personaggi, a romanzare, si direbbe, gli eventi al centro della narrazione, aumenta a livello esponenziale. Inoltre, per quanto riguarda la Shoah, il fenomeno di continua rielaborazione delle sue dinamiche narrative è collegato ad un problema di comprensione (o meglio di in-comprensione) alla base dello stesso genocidio: come si legge nell'Avvertenza al noto saggio Quel che resta di Auschwitz, «il divario riguarda la struttura stessa della testimonianza» (Agamben $1998,8)$. Il filosofo prosegue affermando che:

L’aporia di Auschwitz è, infatti, la stessa aporia della conoscenza storica: la non-coincidenza fra fatti e verità, fra constatazione e comprensione. Tra il voler capire troppo e troppo presto di coloro che hanno spiegazioni per tutto e il rifiuto di capire dei sacralizzatori a buon mercato, indugiare in quello scarto ci è parsa l'unica via praticabile. [...] Tuttavia, poiché, a un certo punto, è apparso evidente che la testimonianza conteneva come sua parte essenziale una lacuna, che i superstiti testimoniavano, cioè, per qualcosa che non poteva essere testimoniato, commentare la loro testimonianza ha significato necessariamente interrogare quella lacuna - 0, piuttosto, provare ad ascoltarla. (Agamben 1998, 8-9; enfasi aggiunte)

La rielaborazione artistica e letteraria della Shoah deriva anche da tale aporia congenita, che tende a voler esser colmata dai destinatari in forme sempre diverse e aggiornate a mano a mano che cro- 
nologicamente ci si allontana dalla realtà storica in cui ebbe luogo lo sterminio. La dimensione del racconto è in parte spontaneamente diventata, per ragioni di distaccamento temporale e di contesto, lo strumento più efficace e diretto a disposizione della contemporaneità per apprendere, documentarsi e produrre memorie circa lo sterminio delle minoranze europee e del popolo ebraico durante la Seconda guerra mondiale. Oltretutto, anche quelle evoluzioni ed emancipazioni sociali proprie dell'individuo contemporaneo (ad esempio una maggiore scolarizzazione, una maggiore tutela dei diritti civili nel sistema legislativo-giudiziario, l'incremento del capitale economico, l'avvento della tecnologia) stanno concorrendo nel plasmare le forme e i toni del racconto di un preciso evento storico direttamente sconosciuto al pubblico - il genocidio ebraico, appunto. Si cerchi di capire meglio il senso di quest'ultima affermazione.

Sempre secondo studi recenti, la Shoah è stata letta e definita anche come un argomento di carattere pop nel senso più etimologico del termine: proprio in virtù del suo essere popolare, la Shoah è stata continuamente riproposta nell'immaginario comune per tutto il secondo Novecento. L'industria hollywoodiana ha contribuito molto a questa democratizzazione popolare del genocidio ebraico. In pochi anni, il cinema è infatti diventato un canale preferenziale per la dimensione pop dell'Olocausto - e gli esempi di pellicole citate sopra ne sono una prova. Nella dimensione cinematografica, un punto di svolta importante è costituito da un film realizzato in bianco e nero in tempi relativamente recenti, che ha raggiunto un tale successo di pubblico da garantirgli ancora oggi un posto privilegiato nella tradizione ed educazione all'Olocausto: Schindler's List.

Il film del 1993 (Universal Pictures) ${ }^{46}$ diretto da Steven Spielberg è un ottimo esempio di prodotto culturale massificato. La pellicola, ${ }^{47}$ che valse a Spielberg l'Oscar per la miglior regia, non fu apprezzata dai sopravvissuti alla deportazione i quali al tempo giudicarono falsa, inappropriata e talvolta offensiva la trasposizione cinematografica della storia di Oskar Schindler. Sebbene infatti il valore del film non sia di tipo documentario (è appunto un film, cioè una realizzazione artistica basata su avvenimenti realmente accaduti), si comprende bene la reazione di coloro che, invece, non accettarono i toni favolistici su cui si basava la trasposizione del regista americano:

46 Per una panoramica critica riguardo il film in merito alla Shoah, cf. Loshitzky 1997. Per un confronto invece più nel dettaglio di quanto si sta affrontando nel presente capitolo, cf. Damiano Garofalo, «Red Coat Reloaded. Shindler's List, l'immaginario della Shoah e la cultura Pop», in Recchia Luciani, Vercelli 2016, 71-86. Cf. inoltre: Cole 2000; Manchel 1995.

47 «Il film di Steven Spielberg è l'opera chiave che segna un cambiamento radicale nella percezione che la collettività degli spettatori - e in primis quella americana, che ha definitivamente finito per inglobarla nel proprio DNA - ha cominciato ad avere della Shoah» (Claudio Gaetani, «Riconosco ergo pop», in Recchia Luciani, Vercelli 2016, 61-2). 
questa è una reazione propria del testimone che, avendo vissuto in prima persona i fatti di cui narra la pellicola, non è uno spettatore come gli altri. Se, da una parte, le istituzioni museali e l'insegnamento dell'Olocausto nelle scuole hanno potenziato i contenuti ed i riferimenti storici in direzione di una consapevolezza più specifica e dal carattere formativo nella contemporaneità, dall'altra la banalizzazione che, viceversa, kolossal cinematografici come Schindler's List hanno indirettamente prodotto nell'immaginario comune si riverbera appunto nella proliferazione e nell'utilizzo generalizzato della «sineddoche Auschwitz» (Recchia Luciani, Vercelli 2016, 11) nel contesto contemporaneo.

La creazione di una iconografia precisa ha suscitato, in primis, un impatto drammatico sul pubblico (si pensi al cappottino rosso della bambina nel film di Spielberg, oppure al ruolo dei nazisti come i 'cattivi' per antonomasia in molti film e romanzi americani), portando però inevitabilmente a standardizzare alcuni rimandi-chiave legati alla sfera dell'Olocausto: facendo leva sull'emotività degli spettatori, gli stessi contenuti sono stati successivamente sdrammatizzati e svuotati, in un certo senso, proprio in virtù della insistente e diffusa presenza allegorica della Shoah nel panorama culturale contemporaneo. Questo fenomeno ha per contro depotenziato e generalizzato infatti il messaggio di cui, in precedenza, detenevano il monopolio le scritture memoriali dei sopravvissuti e alcuni tentativi mediatici di tipo documentario, basati esclusivamente sulla testimonianza storica del genocidio ebraico. Nelle forme artistiche e letterarie di seconda generazione, invece, i toni e stilemi della narrazione tragica si distaccano molto dai tradizionali resoconti compilati dai sopravvissuti, dando luogo a prodotti culturali nuovi, che hanno a loro volta convalidato e confermato il potere 'commerciale' dell'industria della memoria.

E sempre doveroso ricordare che esistono profonde differenze, a livello stilistico e narratologico, nel modo di narrare il genocidio ebraico da parte delle generazioni successive a quella coinvolta direttamente nella Guerra. Si prenda il caso del famoso e molto apprezzato lungometraggio francese Shoah, diretto da Claude Lanzmann e distribuito nel 1985: ${ }^{48}$ sebbene il documentario sia stato acclamato dalla critica e designato vincitore di numerosi premi, la stessa struttura della pellicola - a cominciare dalla sua durata totale di 613 minuti (9 ore e mezza) - si configura come un prodotto dalla visione impegnativa da parte dello spettatore, rivolgendosi automaticamente ad una fascia di pubblico più ristretta (che non vuol dire inesistente 0 necessariamente di nicchia) rispetto al potenziale consumatore medio di un qualsiasi blockbuster cinematografico.

48 Cf. Shoah (1985). Dir. Claude Lanzmann. Les Aleph, Historia Film: Ministère de la Culture de la Republique Française. 
Nonostante l'importanza che tuttora ricopre la letteratura memoriale e la produzione filmica annessa, è indubbio che un nuovo tipo di fruizione, più leggero ed immediato, di facile presa sul pubbliCo, si sia ormai insediato e affiancato alle scritture memoriali nella tradizione e trasmissione dei contenuti relativi allo sterminio nazista degli ebrei: questa tendenza si è generata prima negli Stati Uniti e da qui è stata poi esportata e distribuita su scala mondiale. Il salto concettuale che, sul piano della narratologia, ha stereotipato i testimoni della Shoah rispettivamente nei ruoli di vittime e carnefici, cristallizzandone le forme o aprendoli a nuove declinazioni in molti film e romanzi contemporanei, è un fenomeno complesso, che richiede un'ulteriore, finale valutazione di tipo filosofico e socio-antropologico per poter giustificare a pieno gli esiti e i prodotti di cui si è trattato in questo capitolo, all'interno del bagaglio di esperienze culturali dell'individuo moderno.

\subsection{Il ruolo della vittima}

Nel saggio Modernity and the Holocaust del 1989, ${ }^{49}$ Zygmunt Bauman aveva già intuito quanto l'eredità dell'Olocausto si stesse sviluppando in realtà più secondo una prospettiva sociale che storica: il filosofo ha definito la Shoah una sorta di «test» adatto alla valutazione dei meccanismi identitari e di relazione sociale dell'uomo moderno (Bauman [1989] 2000, 6-30, 175-9). Circa un decennio più tardi, nella postfazione presente nell'edizione successiva del medesimo studio, Bauman ha ampliato la sua analisi considerando anche il problema intrinseco nel dovere di 'onorare la memoria delle vittime' della Shoah quando, tuttavia, il valore storico e antropologico del genocidio ebraico aveva già superato il concetto di vittima in relazione al singolo evento specifico ([1989] 2000, 222-50): il concetto di vittima si è cioè esteso e trasformato in una condizione sociologica più complessa, legata alle dinamiche di trauma e colpa all'interno della società moderna. «Modern modality of being is characterized first and foremost by its endemic unfinishedness» (229): secondo Bauman, questo senso di precarietà e provvisorietà esistenziale ha trasformato l'Olocausto in una sorta di principio ordinatore e regolatore della congenita dose di insoddisfazione presente nell'individuo del nuovo millennio.

Se ormai il fantasma dell'Olocausto si ripropone, irrimediabilmente, nell'auto-riproduzione della condizione di victimhood nella

49 Si fa riferimento alla seconda edizione originale del volume (2000) in cui compare la postfazione a cura dell'autore, pubblicata successivamente alla canonica traduzione italiana del saggio (il Mulino, 1992). 
modernità, ${ }^{50}$ un film come Schindler's List di Spielberg è capace di nutrire lo spettatore del messaggio che, per definizione, caratterizza la vittima in quanto tale - ovvero la necessità di salvarsi:

As the direct experience of the victims recedes and fades, the memory of the Holocaust thins down and condenses into a simple precept of survival: life is about surviving, to succeed in life is to outlive the others. Who survives wins.

This reading of the Holocaust's lesson has been recently displayed - to worldwide acclaim and huge box-office success - in Spielberg's, now well-nigh canonical, image of the Holocaust. [...] Spielberg's film translates the issue of the salvation of humanity into the decision as to who is to live and who to die. (Bauman [1989] 2000, 233-4; enfasi aggiunte)

Preannunciando così parte del pensiero che verrà sviluppato e descritto più avanti da Daniele Giglioli nell'importante studio Critica della vittima (2014), Bauman aveva già percepito che, legando all'insoddisfazione esistenziale dell'individuo il messaggio veicolato dall'Olocausto, si assiste sul piano socio-psicologico alla trasformazione della vittima in eroe: $:^{51}$ un nuovo tipo di individuo regna nella morale occidentale del nuovo millennio, incarnandosi in colui che è esente da colpe e opposto alla visione umanistica dell'homo faber in quanto succube - vittima, appunto - della sua stessa dimensione sociale e specie biologica.

Schindler's List costituisce allora un passaggio importante in quanto l'eroe 'all'americana', nel film di Spielberg, è ancora un'entità attiva e positiva: il protagonista, Oskar Schindler, è capace di redimere l'essere umano dalla condizione di ostaggio in cui «il secolo colpevole» (Giglioli 2014, 21) l’ha circoscritto e confinato, anzi, è proprio lui a liberare da morte certa centinaia di ebrei. La lezione del survival analizzata da Bauman e trasmessa, a suo avviso, dalla pellicola di Spielberg è, non a caso, in antitesi rispetto al sentimento di vergogna che, secondo la lezione di Primo Levi ne I sommersi e i salvati (1986), si realizza invece nel testimone sopravvissuto allo sterminio ('sono vivo solo perché un altro è morto al posto mio'): la pietas con cui la vittima della società moderna è trattata deriva però non dalla

50 «Whichever way you look at it, the ghost of the Holocaust appears self-perpetuating and self-reproducing. It has made itself indispensable to too many to be easily exorcized» (Bauman [1989] 2000, 204).

51 «La vittima è l'eroe del nostro tempo. Essere vittime dà prestigio, impone ascolto, promette e promuove riconoscimento, attiva un potente generatore di identità, diritto, autostima. Immunizza da ogni critica, garantisce innocenza al di là di ogni ragionevole dubbio. Come potrebbe la vittima essere colpevole, e anzi responsabile di qualcosa? Non ha fatto, le è stato fatto» (Giglioli 2014, 9). 
vergogna provata dai sopravvissuti, ma dal capovolgimento della figura dell'eroe, a livello narratologico, in 'eroe poiché vittima' - santificato cioè grazie al suo statuto di vittima. Da un ruolo tradizionalmente attivo e privilegiato, si assiste al ribaltamento dell'eroe in un personaggio passivo e perseguitato, ma che per questo motivo fa scattare una potente identificazione, a livello inconscio, con il destinatario medio di un qualsiasi libro o prodotto cinematografico.

Si noti che il passaggio eroe/vittima a livello narratologico è durato quasi mezzo secolo. Il nuovo eroe moderno ha infatti dovuto prima compiere un passo intermedio per poter autorizzare la vittima ad un cambio di ruolo: in questo senso, il personaggio cinematografico di Oskar Schindler è un anello fondamentale all'interno del processo di slittamento dell'eroe in vittima. Affinché questo spostamento di ruolo possa infatti compiersi e svilupparsi a pieno nell'identità dell'individuo, l'eroe di Schlinder's List (che non è ebreo ma tedesco) in linea con l'immaginario americano di audacia, coraggio e altruismo tipici del personaggio protagonista, si è posto prima come artefice e responsabile della salvezza delle vittime 'effettive' della tragedia storica, ma in seguito è stato rimpiazzato dalle vittime stesse, veri nuovi eroi del nostro millennio.

Per questo motivo, l'Olocausto ha contribuito alla creazione di una evoluzione antropologica unica e peculiare dell'età contemporanea, ed il suo utilizzo paradigmatico e sistematico ha permesso l'istituzionalizzazione e il culto della memoria in senso capitalistico nella società occidentale. Sebbene un tale processo sembri oggi un fenomeno pienamente accettato e globalizzato, le sue radici sono, come abbiamo visto, complesse e intricate, vecchie di almeno sessant'anni e collocabili, in prima origine, nel panorama americano: questo ha colto la sfumatura dell'unicum potenziale veicolata dalla Shoah, sfruttandola poi in molteplici direzioni. Al giorno d'oggi, la parabola $p o p$ dell'Olocausto ha raggiunto l'estensione più estrema della sua area di pertinenza nell'utilizzo di lessico, contesti, e figure stereotipate da un punto di vista culturale a tutto tondo, che ne ha sviluppato la presenza in ambito artistico, letterario e politico con una forte incidenza nella realtà sociale a livelli quasi ossessivi.

La Shoah è un fatto storico e memoriale che continua quindi a funzionare nonostante il passare degli anni allontani sempre di più le nuove generazioni da un passato ormai non più così recente, ma che, si evince, riecheggia insistentemente nelle nuove conflittualità e incongruenze della società e dell'individuo del nuovo Millennio. 


\section{Postmoderno e Shoah}

Sommario 2.1 Le origini. -2.2 Storia e mito. - 2.3 Pulsioni. - 2.4 Mutazioni. - 2.5 Esorcismi.

\subsection{Le origini}

Dopo il relativo silenzio dell'immediato dopoguerra, un silenzio dovuto al bisogno di elaborare il più grande lutto della storia dell'Europa e forse del mondo, l'interesse della società e della cultura verso questi temi si riattiva alla fine degli anni Cinquanta. Le date simboliche che si potrebbero assumere sono il 1958 con la pubblicazione di Se questo è un uomo presso Einaudi e il 1960 con la cattura di Adolf Eichmann. Si tratta in entrambi i casi di processi complessi più che momenti di svolta: Primo Levi trovò le motivazioni per riproporre il testo ad Einaudi proprio perché il clima stava cambiando, aumentavano gli inviti a testimoniare da parte delle scuole e in generale tornava a farsi vivo l'interesse per ciò che era accaduto in quell'epoca; d'altra parte anche la cattura di Adolf Eichmann derivò da un clima mutato progressivamente, e la vita dei latitanti nazisti in Argentina si fece sempre più clandestina, man mano che l'opinione pubblica prendeva coscienza della propria storia passata.

Olivier Guez, nella sua biografia romanzata sulla latitanza di Josef Mengele (2018), racconta efficacemente questo passaggio datandolo 1957. In quell'anno, Eichmann vive a Buenos Aires, la sua esistenza sembra serena e sicura, la sua presenza, come quella di altre figure importanti del nazismo, è stata quanto meno tollerata dal governo Perón fino al 1955, e dopo quella data il latitante ha approfittato comunque dell'instabilità politica per mantenere una posizione 
favorevole. Nella sua cerchia gravita anche lo stesso Mengele, che proprio in quegli anni ha bisogno di un prestito bancario per i suoi investimenti e per farlo deve riacquistare la sua vera identità. Scrive Guez: «Gregor consulta la sua cerchia di amici, come sempre. In Argentina non rischia niente. Gli americani hanno un'unica priorità, combattere contro i sovietici, e i tedeschi non ne vogliono più sapere del nazismo. La guerra è finita» $(2018,66)$ e quindi: «Addio Gregor: nel settembre del 1956 il consolato della Germania occidentale a Buenos Aires rilascia un certificato di stato civile e un estratto di nascita a Josef Mengele» (67).

Ma proprio quell'anno le cose cambiano repentinamente:

Nel novembre 1956 Fritz Bauer, procuratore generale dell'Assia, spicca un mandato di arresto per Adolf Eichmann, "ovunque si trovi". Ebreo, socialdemocratico e omosessuale, prima di fuggire in Scandinavia Bauer è stato internato in un campo di concentramento e la Gestapo lo ha interdetto dai pubblici uffici. Da quando è rientrato in Germania, alla fine degli anni Quaranta, vuole costringere i suoi compatrioti ad affrontare il passato. (68)

La svolta data da Fritz Bauer porta non solo alla cattura di Adolf Eichmann (che poi fu operata dagli israeliani), ma anche al processo di Francoforte iniziato nel 1963. Mentre prima Eichmann era pronto a rivendicare in un'intervista lo sterminio di milioni di ebrei, quasi vantandosi della propria capacità organizzativa, poi sarà l'uomo giustiziato per quelle colpe. A cavallo tra gli anni Cinquanta e Sessanta dunque il clima politico e sociale con cui si guardava agli abominî nazisti muta radicalmente e il mondo deve fare i conti con la propria storia.

Eppure proprio in quegli anni la letteratura inizia a vivere quell'epoca di esplosivo disimpegno che va sotto il nome di postmodernismo. Difficile delinearne con certezza le coordinate cronologiche così come pure le caratteristiche principali, ma è possibile convergere sull'idea che proprio tra la fine degli anni Cinquanta e l'inizio dei Sessanta, la mutazione dell'economia verso un carattere globale, sostenuta dalla diffusione della televisione e dalla pubblicità invasiva, abbia determinato un cambiamento nel paradigma della narrazione.

Com'è noto, secondo Lyotard, la fine dei «grandi racconti» («grand récits») modernisti riguarda innanzitutto l'esaurirsi delle certezze precedenti dovute all'evidenza della storia contemporanea. Tra queste ha un ruolo di primaria importanza l'esperienza concentrazionaria che mina le certezze hegeliane su cui si fondava la modernità e, assieme al crollo del pensiero marxista, attiva la nascita del postmoderno filosofico. Lo spiega con una sintesi tranchante nel Postmoderno spiegato ai bambini, quando scrive: 
Ognuno dei grandi racconti di emancipazione, a qualunque genere abbia dato l'egemonia, è stato per così dire invalidato nel suo fondamento dagli ultimi cinquant'anni.

- Tutto ciò che è reale è razionale, tutto ciò che è razionale è reale: "Auschwitz confuta la dottrina speculativa". Almeno questo crimine, che è reale, non è razionale. (Lyotard 1987, 38)

È la prima di quattro certezze che vengono a cadere nel secondo dopoguerra e via via minano nei loro fondamenti le ideologie (gli altri punti riguardano marxismo, capitalismo, democrazia). Dopo Auschwitz dunque sembra non solo, come nella celebre frase di Adorno, che non si possa fare più poesia, ma che non sia possibile fondare il proprio pensiero sulle certezze della modernità. Tutto ciò comporta, secondo il filosofo francese, il primato dell'esperienza sull'Idea che ribalta l'ordine delle priorità dell'epoca precedente.

In un libro recente (cf. Cinquegrani 2018), cercando di trovare una chiave di lettura per Le Benevole di Jonathan Littell, ho interpretato un passaggio ricorrendo ai tipi psicologici junghiani e identificando così il prototipo del nazista col tipo pensiero estroverso che nella lettura dello psicanalista svizzero fa precedere proprio le idee all'esperienza. Scrive Jung:

costui sarà un uomo che tenderà [...] a far dipendere il complesso delle manifestazioni della sua vita da conclusioni di ordine intellettuale, che in definitiva si orientano nel senso del dato obiettivo, o di fatti obiettivi o di idee universalmente valide. Un tale tipo d'uomo conferisce non solo per sé, ma anche rispetto al suo ambiente, un potere decisivo alla realtà obiettiva, cioè alla sua formula intellettuale orientata verso l'oggetto. In base a questa formula vengono valutati il bene e il male, o viene definito quanto è bello e quanto è brutto. È giusto tutto ciò che corrisponde alla formula, ingiusto tutto ciò che ad essa si oppone, e contingente tutto ciò che ad essa è indifferente. Poiché la formula sembra corrispondere al significato dell'universo, essa diventa anche una formula dell'universo, che deve realizzarsi sempre e dovunque, sia nei particolari che in generale. Come il tipo di pensiero estroverso si sottomette alla sua formula, così deve fare anche il suo ambiente, per il suo proprio bene, giacché chi non lo fa è nel torto, contraddice a un principio universale ed è quindi irragionevole, immorale e senza coscienza. La morale del tipo di pensiero estroverso non gli concede di fare eccezioni. Il suo ideale deve in ogni caso divenire realtà, giacché è, secondo lui, la più pura formulazione della realtà obiettiva e deve pertanto essere anche una verità universalmente valida, indispensabile per il bene dell'umanità. (Jung 1969, 351) 
Questo elemento rende palese come ci sia un nesso preciso per differenza tra il tipo del nazista legato alla formula o all'Idea e il postmoderno, che all'opposto, nella lettura di Lyotard, fa precedere l'esperienza all'Idea.

Tutto ciò comporta almeno due conseguenze notevoli nella ricezione del nazismo in epoca postmoderna. Da una parte, un altro dei teorici più accreditati del postmoderno come Gianni Vattimo riconduce la Fine della modernità all'«esperienza di "fine della storia"» (Vattimo $1985,12)$, stabilendo una cesura nella ricezione della tragica vicenda nazista da parte del postmoderno, che non è più in grado di leggere la storia stessa, se non come esperienza di alterità. D’altra parte leggere e interpretare il mondo, ovvero quella che Brian McHale (1987) chiama «dominante epistemologica» tipica della modernità, non è più una priorità, e viene sostituita dalla «dominante ontologica» che mette in crisi lo statuto stesso di realtà.

Per queste ragioni, quando tra la fine degli anni Cinquanta e l'inizio dei Sessanta, la prepotente riemersione dei temi storici di nazismo e Shoah diviene un'urgenza sociale, la letteratura e in generale la cultura sta percorrendo sentieri altri, che non possono giungere semplicemente a una ricostruzione storica della realtà passata: non possono almeno per quegli autori più tipicamente postmoderni.

\subsection{Storia e mito}

Se la comprensione del mondo presente e passato non è più una priorità, la Storia non è più una chiave di lettura per comprendere la contemporaneità. Il bagaglio di eventi che il nazismo porta con sé diviene quindi altro: diviene una riserva di narrazioni capaci di esplodere, da utilizzare liberamente da scrittori e registi. Il nazismo inizia a fornire storie fuori dalla storia e per farlo la sua identità diventa da reale a psicologica, archetipica, astratta. Non lo è per tutti, va da sé, ma lo è per quegli autori più vicini alla temperie letteraria e culturale del momento.

In epoca postmoderna, come è noto, si appiattisce la differenza tra cultura alta e bassa, e dunque questi fenomeni di riutilizzo dell'esperienza nazista come archetipo o come astrazione travolgono diversi strati della narrazione. Più in basso si sviluppa il fenomeno, moralmente deplorevole se la morale avesse un ruolo in questa epoca, della cosiddetta nazisploitation, parola che si compone dei termini nazi ed exploitation, che letteralmente significa 'sfruttamento', e allude alla priorità concessa al ritorno economico - sfruttando per l'appunto ogni mezzo a disposizione, dal sesso alla violenza, all'horror - piuttosto che al valore artistico o a motivazioni di ordine etico.

Lo svilupparsi di questo vero e proprio genere cinematografico dimostra l'efficacia di alcuni temi affrontati in questo volume: 
1. gli argomenti legati al nazismo, proprio in ragione della loro natura romanzesca, vengono considerati materiale di sfruttamento commerciale di basso profilo;

2. questi temi non sono percepiti come legati alla realtà e perciò al realismo narrativo, ma per lo più vengono utilizzati per la loro capacità di muovere pulsioni inconsce represse, al di là e oltre ogni considerazione etica.

Queste pulsioni sono soprattutto di natura sessuale. Molti sono i casi di sexploitation legati al nazismo. Benché in questi anni si sviluppino teorie psicanalitiche più articolate sembrano essere le teorie freudiane quelle che spiegano più precisamente questi fenomeni. Secondo il padre della psicoanalisi, infatti, i rapporti sadomasochistici sono semplicemente un'evoluzione della coppia naturale tra attivo e passivo. Scrive ad esempio nei Tre saggi sulla teoria sessuale che sadismo e masochismo «detengono tra le perversioni un posto particolare, giacché l'opposizione di attività e passività che ne è alla base appartiene ai caratteri generali della vita sessuale» (Freud [1905] 1988, 53).

Secondo questa teoria il rapporto coercitivo tra vittima e carnefice che è una sorta di gioco di ruolo nella pratica sessuale sarebbe una rappresentazione parossistica di ciò che è insito nella natura stessa dell'uomo. Ma queste due coppie - attivo/passivo e sadismo/ masochismo - si legano alle pulsioni più profonde e istintive dell'uomo. Nel suo percorso, Freud giunge dall'analisi di natura medica della psiche a opere di carattere più sociologico e di psicologia delle masse, e arriva a includere in questo andamento dicotomico anche le strutture sociali e psicologiche che si generano in tempi di guerra.

È del 30 luglio del 1932 la celebre lettera di Albert Einstein a Sigmund Freud nella quale lo scienziato interroga lo psicoanalista sul Perché la guerra?, come titola il saggio che ne è scaturito. La risposta di Freud è chiara fino allo schematismo:

Noi presumiamo che le pulsioni dell'uomo siano soltanto di due specie, quelle che tendono a conservare e a unire - da noi chiamate sia erotiche, esattamente nel senso di Eros nel Simposio di Platone, che sessuali, con estensione voluta del concetto popolare di sessualità, - e quelle che tendono a distruggere e a uccidere; queste ultime le comprendiamo tutte nella denominazione di pulsione aggressiva o distruttiva. (Freud [1905] 1971, 293)

L'andamento dicotomico è molto simile alle precedenti coppie soprattutto nella possibile sovrapposizione tra sadismo e pulsione aggressi- 
va o distruttiva. ${ }^{1}$ Dunque la guerra sarebbe generata da una pulsione aggressiva, che è la stessa che genera il sadismo che è una componente vicina alla funzione sessuale attiva propria di tutti gli uomini.

Attraverso questo semplice sillogismo è possibile comprendere perché il nazismo con la sua aberrante violenza sia ritenuto un mezzo bassamente popolare per stimolare gli istinti sessuali di fruitori privi di scrupoli morali. Il nazismo è infatti ritenuto molto presto un evento simbolico che rappresenta il male assoluto ma anche una versione parossistica del conflitto sadomasochistico. Come sostiene Susan Sontag nel celebre saggio del 1974 Fascinating Fascism, il legame tra sadismo e nazismo è insito alla stessa logica nazista.

Naturalmente molta gente che è eccitata dalle uniformi delle SS non intende con questo dimostrare approvazione per ciò che hanno fatto i nazisti, se pure ne ha una vaga idea. Ciò nonostante, esistono delle correnti di sessualità molto forti e in aumento, abitualmente designate come sadomasochismo, che fanno sembrare erotico il gioco del nazista. Queste fantasie e pratiche sadomasochiste sono diffuse sia tra gli eterosessuali che fra gli omosessuali, anche se l'erotizzazione del nazismo è più visibile tra gli omosessuali. Il sadomasochismo, non la spregiudicatezza, è il più grande segreto sessuale di questi ultimi anni. (Sontag [1974] 1982, 86)

Sontag non parla generalmente di recrudescenza della sessualità attiva che conduce alla gratuità della violenza (come fa, per esempio, Littell nelle Benevole) ma intende il termine sadomasochismo in senso stretto:

Tra sadomasochismo e fascismo c'è una connessione naturale. - Il fascismo è teatro -, come ha detto Genet. E lo stesso vale per il sadomasochismo: essere coinvolti in un atto sessuale sadomasochista significa agire in un teatro sessuale, in una messa in scena della sessualità. I sadomasochisti abituali sono costumisti, coreografi ed interpreti esperti di un dramma che è tanto più eccitante in quanto vietato alla gente comune. Il sadomasochismo sta al sesso come la guerra sta alla vita civile: l'esperienza magnifica. (Dice la Riefenstahl: "Tutto ciò che è realismo puro, tranche de vie, le cose mediocri, quotidiane, non mi interessa...") Se il patto sociale sembra insipido rispetto alla guerra, nello stesso modo scopare e succhiare diventano blandamente piacevoli, e perciò non eccitanti. Il fine a cui tendono tutte le esperienze sessuali, come ha

1 La sovrapposizione di questi due concetti non è per tutti immediata. Anche alcune letture dell'opera di Sade tendono ad associare il sadismo a una funzione razionale piuttosto che a una pulsione aggressiva. Tuttavia per Freud la lettura del sadismo come esasperazione della funzione sessuale attiva non può che essere associata a una pulsione aggressiva. 
sempre scritto Bataille, è la profanazione, l'empietà. Essere "piacevole", come essere civilizzato, significa essere estranei a questa esperienza selvaggia, che ha essenzialmente un carattere di messa in scena. (Sontag [1974] 1982, 86-7)

Questo parallelismo è reso palese da Pier Paolo Pasolini che nel 1975 realizza Salò e le 120 giornate di Sodoma, nel quale la recitazione e la messa in atto delle perversioni delle opere del marchese de Sade è ambientata nella Repubblica di Salò e realizzata da gerarchi fascisti. La corrispondenza diretta tra perversioni sadiche e nazifascismo ha diviso gli intellettuali dell'epoca; presero le distanze per esempio Roland Barthes o Michel Ciment o Italo Calvino. Ma sono le motivazioni messe in campo da quest'ultimo per giustificare la sua stroncatura ad essere proprio rivelatorie della ricezione del nazifascismo:

Per prima cosa devo dire che l'idea di ambientare il romanzo di Sade ai tempi e nei luoghi della repubblica nazi-fascista mi sembra pessima da ogni punto di vista. La terribilità di quel passato che è nella memoria di tanti che l'hanno vissuto non può essere usata come sfondo per una terribilità simbolica, fantastica, costantemente fuori dal verosimile come quella di Sade (e giustamente rappresentata in chiave fantastica da Pasolini). (Calvino 2007, 1933)

Sia o meno un difetto come sostiene Calvino, l'elemento interessante pare essere l'astrazione: la «terribilità simbolica» contro «la terribilità del passato». Roland Barthes, che a sua volta stronca il film di Pasolini, esclude invece l'uso del simbolo («non c'è alcun simbolismo») ma riconosce altre forme di astrazione («da un lato una grossolana analogia (il fascismo, il sadismo), dall'altra la lettera, minuziosa, insistente, esibita, leccata, come la pittura di un primitivo», Barthes 1997, 159). Al di là del giudizio di merito, il teorico francese giunge a una distinzione che riguarda proprio la ricezione del nazifascismo:

Del fascismo non vuole affatto dire il fascismo. C’è il «sistema fascismo» e c’è la «sostanza fascismo». Tanto il sistema richiede un'analisi esatta, una discriminazione ragionata, che deve impedire di considerare come fascista qualunque oppressione, così la sostanza può circolare ovunque; perché in fondo essa è soltanto uno dei modi con cui la 'ragione' politica giunge a colorare la pulsione di morte. (Barthes 1997, 160)

Alla base c'è dunque la pulsione di morte, come già detto più su, ed è questa che viene 'colorata' da ragioni politiche.

In diverse interviste è lo stesso Pasolini a riconoscere il significato di metafora al film. Dice per esempio: «Stavolta il sesso ha una funzione metaforica, e quindi il film non è una favola ma una grande 
metafora, almeno nelle mie intenzioni. [...] il sesso questa volta è la metafora del rapporto tra il potere e chi è sottoposto al potere» (Pasolini [1979] 2001, 3019). In questo senso il dato cronotopico e storico preciso svapora nella sua astrazione: «Lo prendo come metafora del rapporto del potere con chi è subordinato al potere, e quindi vale in realtà per tutti i tempi» (3018). Ciò significa che la storia del nazifascismo, nella ricezione pasoliniana, è piuttosto una metafora che una realtà tragica:

Ma le sue Centoventi giornate di Sodoma non si svolgono appunto a Salò nel 1944?

Sì, a Salò, e a Marzabotto. Ho preso a simbolo di quel potere che trasforma gli individui in oggetti [...] il potere fascista e nella fattispecie il potere repubblichino. Ma, appunto, si tratta di un simbolo. Quel potere arcaico mi facilita la rappresentazione. ([1975] 2001, 2065)

Si potrebbe pensare che questa interpretazione sia legata esclusivamente alla creatività di un regista e scrittore riconosciuto nel panorama internazionale, ma in realtà questa visione spiega molto della ricezione del nazifascismo in questa epoca.

Nel contributo citato di Susan Sontag si fa riferimento ad altri film che rappresentano un legame più o meno esplicito tra sadismo e nazismo:

Se il messaggio del fascismo è stato neutralizzato da un'idea estetica della vita, le sue insegne sono state caricate di sessualità. La trasformazione del fascismo in fatto erotico è riscontrabile in manifestazioni ferventi e affascinanti come Confessioni di una $\mathrm{Ma}$ schera e Sole e Acciaio di Mishima, e in film come Scorpio Rising di Kenneth Anger e nei più recenti e molto meno interessanti La caduta degli Dèi di Visconti e Portiere di Notte di Liliana Cavani. (Sontag [1974] 1982, 84)

Proprio quest'ultimo film, Il portiere di notte di Liliana Cavani, uscito nel 1974, un anno prima di Salò, è stato al centro di una polemica a distanza tra la regista e Primo Levi. Quando, al termine della sua vita, Levi si trova a osservare il modo in cui la società attuale guarda all'esperienza concentrazionaria e nazista, sente la necessità di scrivere quel libro lucido e atroce che è I sommersi e i salvati (1986), proprio per ridiscutere alcuni aspetti del Lager e della sopravvivenza della sua memoria. Ed è in questa circostanza che assume il film di Liliana Cavani come esempio negativo di come la memoria possa essere travisata in favore di ragioni meramente estetiche.

Il film che racconta dell'incontro casuale di una ex internata con un suo aguzzino ad alcuni anni di distanza dall'esperienza del cam- 
po e della loro deliberata scelta di tornare insieme prigionieri delle loro manie, è giudicato da Levi «bello e falso»:

La regista Liliana Cavani, a cui era stato chiesto di esprimere in breve il senso di un suo film bello e falso, ha dichiarato: «Siamo tutti vittime o assassini e accettiamo questi ruoli volontariamente. Solo Sade e Dostoevskij l'hanno compreso bene»; ha detto anche di credere "che in ogni ambiente, in ogni rapporto, ci sia una dinamica vittima-carnefice più o meno chiaramente espressa e generalmente vissuta a livello non cosciente». (Levi [1986] 2016, 1172)

Anche Cavani dunque ricorre a Sade per spiegare quella condizione storica, e con essa l'essenza stessa della psiche umana. Ma Levi commenta:

So che gli assassini sono esistiti, non solo in Germania, e ancora esistono, a riposo o in servizio, e che confonderli con le loro vittime è una malattia morale o un vezzo estetistico o un sinistro segnale di complicità; soprattutto, è un prezioso servigio reso (volutamente o no) ai negatori della verità. (1172)

È facile vedere in queste parole la portata dell'offesa che una vittima reale dell'abominio nazista ricava dal film e da quella dichiarazione di Liliana Cavani, anche conoscendo la quasi proverbiale moderazione dello scrittore torinese. Ma Levi non è un ingenuo e sa bene da dove nasce il film e la conseguente spiegazione della regista, come risulta chiaramente dalle parole che precedono quest'ultima citazione:

Non mi intendo di inconscio e di profondo, ma so che pochi se ne intendono, e che questi pochi sono più cauti; non so, e mi interessa poco sapere, se nel mio profondo si annidi un assassino, ma so che vittima incolpevole sono stato e assassino no. (1172)

Levi sa bene che il punto decisivo è lo iato che si crea tra parlare di vittima e carnefice come funzioni psicologiche e parlarne come condizioni realmente e tragicamente vissute. E sa che la trasformazione di eventi reali in condizioni psicologiche rappresenta un rischio per la conservazione della memoria di quel tempo sciagurato. Non esiste corrispondenza tra le pulsioni rappresentate metaforicamente col nazismo e il nazismo come situazione reale, anzi i due tipi di ricezione di quella memoria paiono tra loro incompatibili.

Nel film la mutazione dei protagonisti è repentina: dall'iniziale spavento precipitano improvvisamente nel vortice della reciproca attrazione, che li conduce all'isolamento dal mondo, alla fame, alla dissoluzione e infine alla morte. Il sesso, anche in questo caso di carattere sadomasochista, pur senza gli eccessi che carezzeranno Sa- 
lò, è l'alfabeto sul quale si declina il rapporto tra vittima e carnefice che assume la maschera storica di ebreo e nazista.

La scena più forte nella quale viene rappresentata la perversione aberrante del nazista, sostanzialmente accettata e accolta dalla donna, è quella che richiama il mito di Salomé: la situazione è raccontata dal punto di vista del ex nazista ma, attraverso uno dei frequenti flashback, mostrata allo spettatore. La protagonista, dopo essersi esibita in una danza sensuale di fronte ai suoi aguzzini nazisti, riceve una scatola in dono nella quale c'è la testa di una persona rispetto alla quale aveva precedentemente espresso la sua avversione. In questo modo diviene lei stessa responsabile di quella morte, secondo un procedimento tipicamente sadiano e al tempo stesso tipicamente nazista.

Il ricorso al mito biblico ed ai suoi personaggi da sovrapporre ai protagonisti del film rende evidente il tentativo di risalire agli archetipi dell'umanità anziché cercare di circoscrivere un momento storico. Il mito di Salomé, del resto, è sempre stato reinterpretato come simbolo, per lo più negativo, della donna violenta e senza scrupoli del «fiore che agita la tempesta» come la definisce Flaubert nell'Erodiade. Nel film questo passaggio è enfatizzato, la scena è la più nota dell'intera pellicola, mentre lo spettatore assiste allo svolgersi qui e ora attraverso un flashback, il narratore Max la commenta rendendo esplicito il riferimento al mito. Ma il tragico sublime al quale allude è morto da secoli, almeno dall'epoca romantica, e resta una sovrainterpretazione di carattere per lo più psicanalitico, come avviene nei casi più noti e diffusi come quello di Edipo.

Questo procedimento porta a un progressivo svaporare della realtà storica sopraffatta da istanze diverse, simboliche come accadeva per Pasolini o pulsionali per Il portiere di notte. In ogni caso il retrocedere della storia lascia il campo libero a una ridefinizione più aperta, funzionale e romanzesca di questi temi. Il fenomeno non è nuovo, basti pensare alle varie saghe western e al confronto tra indiani e cowboy che pure alludono allo spaventoso ed enorme genocidio dei nativi americani, ma diventato per noi un gioco senza più legame con la storia. Per quanto riguarda le storie più recenti del nazismo, ovviamente non si è ancora giunti a questo punto, ma in quest'epoca si passa dal tentativo di ricostruire la realtà storica a quello di trasformare l'assoluto nazista in archetipi universalmente riconosciuti.

La liberazione delle pulsioni si lega al cedere delle «azioni di smorzamento» dovute alla resistenza della morale o della legge nel mondo civile che ovviamente in Lager non sussisteva, come sostiene anche Primo Levi parlando delle grandi differenze sociali in Se questo è un uomo:

Questa divisione è molto meno evidente nella vita comune; in questa non accade spesso che un uomo si perda, perché normalmente l'uomo non è solo, e, nel suo salire e nel suo discendere, è le- 
gato al destino dei suoi vicini; per cui è eccezionale che qualcuno cresca senza limiti in potenza, o discenda con continuità di sconfitta in sconfitta fino alla rovina. Inoltre ognuno possiede di solito riserve tali, spirituali, fisiche e anche pecuniarie, che l'evento di un naufragio, di una insufficienza davanti alla vita, assume una anche minore probabilità. Si aggiunga ancora che una sensibile azione di smorzamento è esercitata dalla legge, e dal senso morale, che è legge interna; viene infatti considerato tanto più civile un paese, quanto più savie ed efficienti vi sono quelle leggi che impediscono al misero di essere troppo misero, e al potente di essere troppo potente.

Ma in Lager avviene altrimenti: qui la lotta per sopravvivere è senza remissione, perché ognuno è disperatamente ferocemente solo. (Levi [1958] 2016, 207)

A partire da questa semplice verità - che cioè ognuno è «ferocemente solo» e dunque privo di leggi, di morale, di usi sociali che lo salvaguardino - molta narrazione di questo periodo priva i fatti di verità storica e di drammaticità umana, concentrandosi sulle componenti psicologiche e pulsionali, e trasformando così la dinamica tra vittima e carnefice in una condizione psicologica narrativamente produttiva o, peggio, morbosamente appetibile.

\subsection{Pulsioni}

In quegli stessi anni, nel 1973, in America, Thomas Pynchon pubblica L'arcobaleno della gravità, riconosciuto ben presto come capolavoro del postmoderno: il tema, pur difficile da circoscrivere, gravita attorno al nazismo. Il titolo stesso allude al razzo V2 costruito dai tedeschi per colpire Londra durante la Seconda guerra mondiale. Protagonista è Tyrone Slothrop che, a causa di uno strano riflesso pavloviano indotto, ha un'erezione nel punto in cui cadrà il razzo V2. Questo nucleo narrativo porta con sé una enorme e quasi incontrollabile esuberanza narrativa che coinvolge innumerevoli personaggi e situazioni che tendono a sfuggire a ogni classificazione ma che restano aggrappati a questo elemento portante.

Nonostante la complessità narrativa non è difficile individuare lo spunto che ha portato alla costruzione di questo libro. Tra le molte e diverse definizioni di postmoderno, resta famosa la scherzosa ma efficace frase di Umberto Eco nelle Postille al Nome della rosa:

La risposta post-moderna al moderno consiste nel riconoscere che il passato, visto che non può essere distrutto, perché la sua distruzione porta al silenzio, deve essere rivisitato: con ironia, in modo non innocente. Penso all'atteggiamento post-moderno come a 
quello di chi ami una donna, molto colta, e che sappia che non può dirle «ti amo disperatamente», perché lui sa che lei sa (e che lei sa che lui sa) che queste frasi le ha già scritte Liala. Tuttavia c'è una soluzione. Potrà dire: «Come direbbe Liala, ti amo disperatamente». A questo punto, avendo evitato la falsa innocenza, avendo detto chiaramente che non si può più parlare in modo innocente, costui avrà però detto alla donna ciò che voleva dirle: che la ama, ma che la ama in un'epoca di innocenza perduta. Se la donna sta al gioco, avrà ricevuto una dichiarazione d'amore, ugualmente. Nessuno dei due interlocutori si sentirà innocente, entrambi avranno accettato la sfida del passato, del già detto che non si può eliminare, entrambi giocheranno coscientemente e con piacere al gioco dell'ironia... Ma entrambi saranno riusciti ancora una volta a parlare d'amore. (Eco 1984, 530)

Il postmoderno deriva da uno sguardo non innocente, con continue interferenze culturali che impediscono la spontaneità della percezione della vita: non è possibile parlare d'amore senza riferirsi a Liala o a centinaia di altre figure «in un'epoca di innocenza perduta». Resta dunque il «gioco dell'ironia» di chi accetta «la sfida del passato» col proprio bagaglio di cultura.

Anche L'arcobaleno della gravità si potrebbe spiegare a partire da questo elemento, o almeno è possibile comprendere lo spunto narrativo da cui nasce: che è rappresentato dal razzo V2, antesignano dei missili balistici che hanno segnato la storia successiva. Sebbene l'efficacia militare del missile sia dubbia, il suo impatto emotivo doveva essere molto forte. Anche oggi di fronte a questo monolite di 14 metri e di 13 tonnellate, l'immaginario dello spettatore non resta indifferente. Ed è chiaro che ognuno di noi, anche con una cultura media 0 addirittura limitata, associa più o meno inconsciamente il razzo a un simbolo fallico descritto da Sigmund Freud. Lo sguardo non innocente raccontato da Eco, dunque, dovrà fare i conti con questa frase: «Come direbbe Freud, il missile uccide». Tutto ciò comporta una serie di conseguenze:

- il missile è associato a un simbolo fallico («come direbbe Freud»);

- ma «il missile uccide» mentre il fallo dovrebbe generare la vita;

- le pulsioni sessuali (fallo) e le pulsioni di morte (missile) convergono nello stesso oggetto.

Da queste considerazioni deriva lo spunto narrativo che altrimenti parrebbe incomprensibile o delirante, ovvero l'associazione tra l'erezione del protagonista e l'impatto distruttivo del razzo. Lo sviluppo narrativo porta ovviamente molto oltre l'autore, ma tutto si concatena a questo semplice spunto primario: da qui nasce ad esempio la necessità di spiegare come questo sia possibile, e quindi ci sono lunghe digressioni sul funzionamento dei riflessi pavloviani; oppure 
c'è l'esigenza di costruire situazioni che articolino ulteriormente questo legame degli opposti, come i rapporti sadomasochistici già visti per Pasolini; o ancora resiste l'intenzione di sviluppare la parabola narrativa costruendo un finale.

La sovrapposizione di Eros e Thanatos nel razzo-fallo è resa esplicita più volte all'interno del testo. Addirittura il suo impatto distruttivo è associato a un orgasmo:

Una volta, Katje amava pensare al razzo come a un pavone che, nella fase di corteggiamento, faceva la ruota... vedeva la ruota aprirsi a ventaglio nei colori che s'agitavano all'interno della fiamma, mentre il razzo si staccava dalla rampa di lancio - rosso scarlatto, arancione, verde iridescente... per qualcuno, fra i tedeschi, perfino tra le SS, il razzo era der Pfau. «Pfau Zwei.» La sua ascesa programmata avveniva secondo un ritmo amoroso... arrivati al Brennschluss, il rito era compiuto - l'equivalente puramente femminile del Razzo, il punto zero al centro del bersaglio, si era concesso. Tutto quello che seguiva, avveniva conformemente alle leggi della balistica. Il Razzo si muoveva inerme. Qualcos'altro aveva assunto il comando. Qualcosa non contenuto nel suo progetto originale.

Per Katje il grande arco tracciato nel vuoto era una chiara allusione a certe voglie segrete che animavano tanto il pianeta quanto lei, e quanto Quelli che la usavano... raggiungeva l'apice e poi si tuffava giù, ardente, verso l'orgasmo terminale... ovviamente non era una cosa di cui potesse parlare con Slothrop. (Pynchon 2017, 315)

È chiara la convergenza delle pulsioni nel volo del razzo: trattando dell'economia pulsionale, però, la realtà storica svanisce anche in questo caso e nulla di ciò che leggiamo può avvicinarci alla realtà di quel tempo. Il tema è un altro, più ampio e forse universale, e il nazismo è solo il correlativo oggettivo di questo tema.

Nella parabola del lettore e del personaggio, dopo l'associazione tra razzo e fallo, è necessario ritornare a vedere nell'immagine di quell'arma da guerra il missile e non più ciò che Freud ci impone di vedere. O almeno le due immagini devono tornare ad essere fuse. Slothrop perciò, in conclusione del romanzo, diventa, in un certo senso, il razzo stesso, lo disegna ossessivamente dal basso, in soggettiva, e pensa:

E adesso, nella Zona, più tardi, nello stesso giorno in cui lui era diventato un crocevia, dopo un acquazzone di cui non aveva ricordo, Slothrop vede uno spesso arcobaleno, un grosso pene uscito dal pube delle nuvole penetrare giù nella Terra, nelle sue valli verdi e umide, e sente il petto gonfiarsi, e resta lì in lacrime, senza un solo pensiero in testa, sentendosi tutt'uno con la natura... (Pynchon 2017, 857) 
Il punto è ritornare alla natura, al fatto in sé: accettare gli opposti come categorie del caos che genera la vita. Chi non riconosce la natura di questi opposti - lo si dice fin dall'inizio del romanzo - precipita nella paranoia:

Pavlov era affascinato dall'«idea degli opposti». La si potrebbe definire un ammasso di cellule, localizzate in un punto imprecisato della corteccia celebrale. Queste cellule servono a distinguere il piacere dal dolore, la luce dall'oscurità, il dominio dalla sottomissione... Però quando in qualche modo l'idea degli opposti viene indebolita nei soggetti studiati - facendo patire loro la fame, traumatizzandoli, percuotendoli, castrandoli, facendoli precipitare in una delle fasi transmarginali, oltre i confini del loro io cosciente, oltre la fase «di pareggio» e la fase «paradossale» - all'improvviso ci si ritrova con un paziente paranoico che vorrebbe essere un padrone, eppure si sente uno schiavo... che vorrebbe essere amato, però soffre per l'indifferenza del mondo. «Io credo che sia proprio la fase ultraparadossale» scrive Pavlov a Janet, «a indebolire l'idea degli opposti nei nostri pazienti». Pazzi, paranoici, maniaci, schizoidi, idioti in senso morale... (74-5)

L'esplosione schizoide depotenzia la tenuta morale: è questa la descrizione dei personaggi postmoderni e della loro lettura del nazismo come universo pulsionale. L'assenza di una riconoscibile struttura morale e di una opposizione tra bene e male che ne sarebbe la logica conseguenza allontana questa narrazione pulsionale dalle strutture tipiche del romance che invece, come si è visto e si vedrà, innervano narrazioni più recenti. Il postmoderno appare oggi distante proprio perché nella letteratura degli ultimi anni i principi pulsionali indietreggiano a favore di una strutturale morale o moraleggiante:

«Il dottor Jampf non è mai esistito» sostiene Mickey Wurtry-Wurtry, noto psicanalista di fama mondiale, «Jampf era solo una finzione, gli serviva a trovare una spiegazione alla sensazione che provava nei genitali, così dolorosa, così immediata, ogni volta che uno di quei razzi esplodeva in cielo... gli serviva a negare quello che non poteva assolutamente ammettere: il fatto di provare un amore, un amore fisico, per la morte sua e della sua razza». (1009)

L'evanescenza finale di Slothorp («Slothorp è stato demolito, i suoi pezzi dispersi», 1009) allude proprio a un cambio di paradigma: il progressivo logorarsi di questo universo pulsionale che Pynchon chiama entropico, per dare spazio ai significati morali. Nel $2000 \mathrm{Je}$ an Baudrillard nell'Illusione dell'immortalità parla proprio di questo passaggio: 
Dopo la grande rivoluzione realizzata nel processo evolutivo - l'avvento della differenziazione sessuale e della morte - stiamo subendo ora una grande involuzione, il cui scopo, attraverso la clonazione e molte altre tecniche all'avanguardia, è quello di liberarci proprio dal sesso e dalla morte. Da che eravamo un tempo creature viventi che hanno lottato assiduamente per milioni di anni per affrancarci da questa specie di incesto e di primitiva entropia, oggi siamo diventati, grazie alle stesse scoperte scientifiche, esseri incoscienti che inseguono il sogno di ricreare precisamente le condizioni da cui ci siamo con tanta fatica liberati. (Baudrillard 2007, 23-4)

Liberarci del sesso e della morte significa liberarci delle basi dell'universo pulsionale raccontato da Freud e giungere a una forma di depressione latente. È questo il tema principale di un altro dei grandi autori del postmoderno: Don DeLillo.

Nel 1978 l'autore newyorkese pubblica il romanzo Running Dog che gravita ancora attorno a temi legati al nazismo interpretato come una convergenza di pulsioni sessuali e pulsioni di morte. In questo caso la sovrapposizione degli opposti è resa ancora più palese: al centro della narrazione infatti è un misterioso video che a quanto sembra ritrarrebbe Hitler in persona nel suo bunker mentre partecipa a un'orgia o qualcosa di simile. Le voci attorno a questo filmato suscitano grande trepidazione tra collezionisti d'arte, appassionati, ricchi amatori che ne fiutano il valore, fino a coinvolgere anche la CIA. Da una sorta di sopore annoiato sembra che una parte della società americana si risvegli grazie a un oggetto che suscita un interesse voyeuristico proprio perché il più diabolico rappresentante del male e della morte diviene anche oggetto di pulsioni sessuali. Ed è questa convergenza apparentemente assurda a determinare gran parte dell'attenzione che la narrativa riserva a questo periodo: «Vedi, è che c'è un fascino incredibile dietro tutto questo. Tutta l'era nazista. Non se ne ha mai abbastanza. Qualsiasi cosa sia nazista equivale a essere erotica» (DeLillo 2005, 53); «Affascinante. Una parola interessante. Viene dal latino fascinus. Un amuleto a forma di fallo. Una parola da cui deriva anche il termine fascismo» (147). Del resto sull'ambiguità di questi termini si muoveva anche il titolo del saggio già citato di Susan Sontag Fascinating Fascism.

Ma la questione affrontata da DeLillo non riguarda soltanto la ricezione del nazismo ma si focalizza anche e soprattutto sul presente. Il finale del romanzo infatti disattiva totalmente l'eccitazione che il presunto video porno di Hitler aveva suscitato, perché una volta raggiunto e visto, si scopre che in realtà non c'era nulla di pornografico, ma era semplicemente una situazione qualsiasi della vita quotidiana. L'effetto è quasi annichilente, la trepidazione e l'attesa svaniscono in un attimo, le pulsioni, attivate nella lunga attesa, disperse di fronte alla semplice verità. 
Il lavoro di DeLillo è costantemente centrato su questa dicotomia tra un mondo, passato o lontano, in cui le pulsioni mantengono la loro vitalità e il presente in cui le pulsioni non esistono più o sono inevitabilmente e pericolosamente annichilite: in Underworld per esempio sono le armi a trasformarsi in rifiuti inerti, nel recente Zero K è il terrorismo a suscitare un ravvivarsi (degenerato) delle pulsioni, Cosmopolis è la rappresentazione dello spreco delle pulsioni in un uomo che rappresenta la ricca società finanziaria occidentale, in Rumore bianco, invece, è ancora il nazismo (cf. Mazzarella 2017; Cinquegrani 2019b).

Nel romanzo del 1985 che ha dato la notorietà internazionale a DeLillo il protagonista Jack Gladney è l'ideatore e il fondatore del primo Dipartimento di studi hitleriani degli Stati Uniti. La sua fondazione, si spiega nelle prime pagine, crea un clima di eccitazione:

Io sono il preside del dipartimento di studi hitleriani presso il College-on-the-Hill. Sono stato io, nel marzo del '68, a inventare gli studi hitleriani in America del nord. Era una giornata fredda e luminosa, con venti intermittenti da est. Quando feci balenare nel rettore l'idea che avremmo potuto edificare un intero dipartimento attorno alla vita e all'opera di Hitler, fu lesto a coglierne le possibilità. Il successo fu immediato ed elettrizzante. Il rettore divenne consigliere per Nixon, Ford e Carter prima di morire su uno skilift in Austria. (DeLillo 2014, 6-7)

L'idea è accolta con entusiasmo, il successo è immediato e soprattutto elettrizzante («an immediate and electrifying success», DeLillo 1999, 4), proprio perché Hitler e il nazismo scatenano le nostre pulsioni. Ma a mano a mano che la storia procede si comprende che tutto questo non può funzionare. Iniziano ad esserci delle crepe, per esempio Jack prende coscienza che non sarà mai un ottimo studioso di Hitler perché non conosce il tedesco. L'avvicinamento a questo personaggio e questi temi sarà sempre parziale, e via via si comprenderà che i protagonisti vivono uno stato di depressione permanente che cercano di superare attraverso fantomatici farmaci sperimentali. È l'angoscia della morte a prevalere nel tempo presente, e l'angoscia è dovuta proprio all'assenza o alla rimozione della morte stessa: «Come puoi essere sicura che è della morte che provi paura? La morte è un fatto vaghissimo. Nessuno sa cosa sia, che sensazioni provochi o come sia fatta» (DeLillo 2014, 236).

Questa dicotomia, accentuata dalla rimozione della morte stessa tipica della nostra società, è spiegata bene da Jean Baudrillard, in relazione al terrorismo:

I terroristi sono riusciti a fare della loro stessa morte un'arma assoluta contro un sistema che vive dell'esclusione della morte, che ha eretto a ideale l'azzeramento della morte, la zero-morte. (Baudrillard 2002, 22-3) 
Oppure, più genericamente, in relazione all'angoscia che provoca, come scrive nel 1976, cioè negli anni che qui si stanno analizzando:

La sua [della morte] scomparsa nell'immaginario non è che il segno della sua interiorizzazione psicologica, quando la morte cessa di essere la grande mietitrice per diventare l'angoscia della morte. (Baudrillard 2015, 160)

La scomparsa della morte suscita quindi quell'angoscia vaga ma opprimente che rappresenta la nostra società. Al polo opposto c'è Hitler che invece quella morte cercava tragicamente e sciaguratamente. Nell'analisi destabilizzante di DeLillo c'è un passo particolarmente significativo in cui uno dei personaggi minori si rivolge a Jack in questi termini:

- La gente pensa che io sia stramba, - continuò. - E certamente ho una teoria stramba circa la paura umana. Immagina te stesso, Jack, uomo tutto casa e famiglia, persona sedentaria, che si trova improvvisamente a camminare nel folto di una foresta. Con la coda dell'occhio cogli qualcosa. Prima di avere ulteriori informazioni, sai che si tratta di qualcosa di molto grosso, che non trova posto nel tuo attuale schema di riferimento. Un difetto nel quadro del mondo. Uno di voi due non dovrebbe essere lì. Poi la suddetta cosa diventa pienamente visibile. È un grizzly, enorme, di un bruno lucente, barcolla, cola bava dalle zanne scoperte. Tu, Jack, non hai mai visto un animale grosso nella foresta. La visione di questo grizzly ti risulta così elettrizzantemente strana da darti un senso rinnovato di te stesso, una nuova consapevolezza dell'io nei termini di una situazione unica e orripilante. Vedi te stesso in un modo nuovo e intenso. Ti riscopri. Ti vedi in piena luce nell'imminenza di venire smembrato. La belva, retta sulle zampe posteriori, ti ha reso capace di vedere come sei veramente per la prima volta, fuori dall'ambiente famigliare, solo, separato, integro. La definizione che diamo di questo complesso procedimento è: paura. (DeLillo 2014, 273)

In questo passo si comprende bene la differenza tra l'angoscia della morte provocata dalla sua assenza e la paura che invece è causata dalla sua imminenza o plausibilità e che rappresenta una risorsa per la conoscenza di sé. Tutto ciò, ovviamente, non ha nulla a che fare con un elogio dell'epoca nazista, che sarebbe totalmente impensabile, ma spiega piuttosto l'interesse morboso con cui nei decenni successivi si è guardato a quel periodo. Hitler assomiglia a quell'orso, ed è evidente che nessuno vorrebbe trovarsi in quella situazione, come del resto si capisce dalla reazione di Jack («Devo rischiare la morte correndo in una strada piena di curve? Oppure pensi che deb- 
ba andare a scalare le montagne nei weekend?», DeLillo 2014, 274), eppure è altrettanto plausibile che chiunque guarderebbe a quella scena dell'orso con un interesse morboso.

La visione del grizzly risulterebbe «elettrizzantemente strana» («electrifyingly strange») esattamente come l'idea dell'istituzione di un dipartimento di studi hitleriani all'università ha un effetto «elettrizzante» («electrifying»). Questo effetto di attrazione morbosa è dovuto proprio all'attivarsi improvviso, imprevisto e irrazionale delle pulsioni di morte che si confondono con le pulsioni sessuali.

In questo modo il lettore di questo periodo si rivolge alle storie di argomento nazista, prima di riflettere sul contesto storico che l'ha generata e sulle complesse questioni morali che presenta. L'esplosione romanzesca d'epoca postmoderna è possibile dunque a partire da questa disposizione del lettore.

\subsection{Mutazioni}

Nel 1962 Philip K. Dick pubblica The Man in the High Castle, un altro dei capolavori del postmoderno, tradotto goffamente in italiano come La svastica sul sole. Il tema è ancora il nazismo, ma questa volta è declinato in un universo distopico nel quale tedeschi e giapponesi hanno vinto la Seconda guerra mondiale e dominano l'America.

Come in tutti i romanzi distopici, a Dick interessa più che il fatto storico da cui prende spunto, ciò che esso dimostra nelle ricadute sulla società attuale. In questo caso l'universo nazista rappresenta piuttosto un paradosso romanzesco, nel quale, secondo la visione che i tedeschi incarnano, la realtà è popolata da idee che superano la contingenza del presente. Come si è detto essi rappresentano il prototipo junghiano del pensiero estroverso, ovvero chi dà valore soltanto alla «formula intellettuale orientata verso l'oggetto». Si spiega nel romanzo:

La loro visione: è cosmica. Non un uomo qua, un bambino là, ma un'astrazione: la razza, la terra. Volk. Land. Blut. Ehre. Non l'onore degli uomini degni d'onore, ma l'Ehre stesso; per loro l'astratto è reale, e il reale è invisibile. Die Gute, ma non gli uomini buoni, non quest'uomo buono. È il loro senso dello spazio e del tempo. Essi vedono attraverso il 'qui' e 'ora', nell'enorme e nero abisso che c'è al di là, nell'immutabile. E questo è fatale alla vita. Perché alla fine non ci sarà più vita. (Dick 2019, 66)

L'interpretazione del polimorfismo della vita come idea monolitica e la trasformazione dell'essere umano in un concetto è alla base di un sistema narrativo tradizionale che era tipico della precedente epoca modernista. Si legga ad esempio come parla dei personaggi Luigi Pi- 
randello, un autore tipicamente modernista, nella prefazione ai Sei personaggi in cerca d'autore nella quale definisce gli scrittori filosofi - e perciò anche se stesso - come coloro i quali «sentono un più profondo bisogno spirituale, per cui non ammettono figure, vicende, paesaggi che non s'imbevano, per così dire, d'un particolare senso della vita, e non acquistino con esso un valore universale» (Pirandello [1925] 2016, 328). Questi personaggi, perciò, tendono a fuggire dalla contingenza della vita per astrarsi e fissarsi in un'idea, esattamente come prevede il metodo definito nazista nel libro di Dick.

Oltre che un'aberrazione storica, un incubo, quindi, l'universo distopico della Svastica sul sole diviene uno spazio romanzesco, nel quale, come lo stesso Dick riconosce, si muovono più archetipi che persone, più idee che uomini. E questo atteggiamento affonda le sue radici nella morte di Dio, che impone all'uomo, come spiega Nietzsche nell'aforisma 125 della Gaia scienza, di trasformarsi a sua volta in una divinità. Come la morte di Dio provoca l'avvio della modernità e del modernismo letterario - si pensi a come Pirandello parafrasi questo passo di Nietzsche nel discorso del lanternino del Fu Mattia Pascal - così conduce alla dissennata hybris nazista che si snatura, dice Dick, nella creazione di un ego sconfinato, di un gigante sghembo:

Vogliono essere gli agenti, non le vittime della storia. Si identificano con la potenza di Dio e credono di essere simili a dei. Questa è la loro pazzia di fondo. Sono sopraffatti da qualche archetipo; il loro ego si è dilatato psichicamente a tal punto che non sanno più dire dove cominciano loro e dove finisce la divinità. Non è hybris, non è orgoglio; è l'ego gonfiato a dismisura, fino all'estremo... la confusione tra colui che adora e colui che è adorato. L'uomo non ha divorato Dio; Dio ha divorato l'uomo. (Dick 2019, 66)

Spazio romanzesco e verità storica dunque convergono definendo i canoni della modernità. Lo storytelling distopico si rivolge a temi relativi al nazismo proprio in virtù di questo. Ma, come s'è detto, Dick è il campione del postmoderno, e quindi è oltre la modernità: al centro della sua narrativa c'è non solo la messa in crisi dell'identità individuale e sociale ma anche la deriva ontologica di cui ampiamente parla Brian McHale. La contaminazione tra possibile e impossibile che Doležel riconduce a Kafka in questo romanzo assume proporzioni destabilizzanti. Scrive lo studioso ceco:

Il mondo ibrido, creato da Franz Kafka, ha avuto un formidabile impatto sulla fiction modernista e postmodernista. Poiché la frontiera che divide il mondo finzionale del mito classico è annullata, il mondo ibrido è una coesistenza, in uno spazio funzionale unificato, di entità funzionali (persone, eventi) fisicamente possibili e 
fisicamente impossibili. [...] Le condizioni aletiche del mondo ibrido ci impongono di abbandonare l'opposizione naturale/soprannaturale. (Doležel 1999, 190)

Il mondo creato da Dick, che ha dei punti di contatto - fin dal titolo - col Castello di Kafka, ha una duplice natura ibrida. Da una parte il presente narrativo racconta il mondo postbellico dominato dai nazisti, dall'altra un famigerato romanzo di fiction utopica di cui si parla nella diegesi narrativa descrive un mondo in cui i nazisti hanno perso e regna la democrazia. Per qualsiasi lettore è evidente il paradosso ibrido di entrambi i mondi: la realtà narrativa risulta una finzione al lettore mentre il romanzo nel romanzo appare più vicino alla realtà (alla quale tuttavia non si sovrappone esattamente, date le molte differenze con la storia). Lo smarrimento ontologico è evidente, le «condizioni aletiche» (possibile, impossibile, necessario) fanno perdere le certezze.

L'uomo contemporaneo si rivolge all'epoca nazista come a un mondo incredibile, impossibile. Questo ovviamente non ha nulla a che fare col negazionismo, ma con un abisso di aberrazione legalizzato che è fortunatamente distantissimo dalla quotidianità che vive. L'uomo contemporaneo non può che essere attonito, non può che chiedersi come sia stato possibile - e le risposte, in tal senso, sono innumerevoli - e cercare scappatoie morali per mantenere intatta l'idea stessa di essere umano. Le «condizioni aletiche» di cui parla Doležel si replicano: è davvero stata possibile una cosa impossibile e impensabile? Se è possibile l'impossibile cosa è autentico, necessario, certo? Ancora una volta - e con più evidenza di prima - la sovrapposizione possibile tra nazismo e postmoderno si afferma in questo passaggio.

Altrettanto interessante è vedere cosa accade dopo, perché resta significativo verificare come il tema qui affrontato sia rappresentativo di un'epoca. La svastica sul sole ha almeno due eredi diretti: da una parte Il complotto contro l'America di Philip Roth che si ispira direttamente al romanzo di Dick pur essendo, ovviamente, molto diverso, dall'altra The Man in the High Castle è diventata recentemente una serie TV omonima prodotta da Amazon Prime Video. Il raffronto di questi due eredi col capostipite spiega facilmente la mutazione che si è avuta nella narrativa dal 1962, anno del primo libro, al 2004, anno del Complotto di Roth, fino al 2015 quando esce la prima stagione della fortunata serie televisiva.

Il romanzo di Roth è un altro degli esempi più noti di narrativa distopica. Come nel precedente di Dick il nazismo dilaga in America, in questo caso non per la conquista territoriale da parte dei tedeschi ma per una sciagurata alleanza del nuovo presidente, Charles Lindbergh, con i tedeschi di Hitler.

Rispetto al romanzo di Dick, la prossimità alla situazione reale è molto maggiore. Molte sono le tracce che lo dimostrano, a partire dal 
giovane protagonista, Philip, che ha la stessa età dell'autore all'epoca e anche la stessa famiglia. Anche Lindbergh, per quanto in un ruolo diverso, è una persona reale, con la stessa fama e le stesse simpatie naziste del personaggio del romanzo. Anche l'innesco narrativo, il complotto che dà il titolo al libro, è ispirato alla tragica perdita del figlio del famoso aviatore. Molti altri sono i personaggi ispirati a fatti reali, come Walter Winchell, celebre speaker radiofonico dell'epoca, che nel romanzo si oppone all'avanzata filonazista fino a soccombere.

Il romanzo non prevede ambiguità su questa adesione al vero, come dimostra in modo lampante il paratesto. Alla fine del testo si legge un Poscritto composto da: «Nota per il lettore» che riporta una bibliografia di libri storici nei quali si narrano episodi e si ricostruiscono personalità riconducibili, sia pure nella manomissione finzionale, all'intreccio del romanzo; «Una vera cronologia dei personaggi principali», che permette al lettore di rintracciare il confine tra realtà e finzione; «Un po' di documentazione» che raccoglie un discorso del vero Lindbergh e un brano di un libro storico su di lui, dal quale emerge inconfutabilmente il suo profilo filonazista.

In virtù di questi materiali risulta ancora più evidente lo scopo del libro, che non è, come nel caso precedente, quello di ricorrere a questi temi per ricostruire in metafora i tratti principali della letteratura postmoderna, come lo smarrimento ontologico o l'autenticità irraggiungibile, quanto piuttosto quello di utilizzare la distopia per comunicare un messaggio chiaro e inequivocabile sulla realtà presente. L'antisemitismo, quindi, non è più un territorio di autocoscienza, è piuttosto un pericolo reale, forte e presente, nella storia e nella società attuale. Il messaggio, in questo modo, sostituisce la complessità della narrazione postmoderna.

Il primo capitolo del libro si intitola «Votate per Lindberg o votate per la guerra» e spiega le ragioni della vittoria dell'aviatore che riguardano la pretesa di un'ampia frangia del popolo statunitense di restare fuori dal conflitto bellico che necessariamente avrebbe portato perdite ingenti tra i giovani. È un tema reale, quello dell'impegno o meno contro l'aberrazione che avveniva altrove, un tema che ha segnato la campagna elettorale del 1940, anche se nella realtà ha portato all'inaspettato terzo mandato di Roosevelt. Nel Poscritto si legge infatti che il presidente era «accusato dalla destra repubblicana di essere "un guerrafondaio"» (Roth 2014, 397), stabilendo così un nesso diretto tra le motivazioni politiche e la storia dispotica: il what if ha dunque un messaggio chiaramente politico in un'epoca in cui ritorna la percezione della necessità dell'impegno proprio in contrasto col disimpegno postmoderno.

Nell'«Introduzione» a La letteratura circostante, Gianluigi Simonetti facendo un quadro della letteratura che va oltre i confini nazionali scrive: 
Il sogno dell'industria culturale odierna consiste probabilmente in un'opera che risolva questa e altre contraddizioni [sull'identità dell'individuo e della società] sostituendo l'aspirazione moderna a un'arte come infinito possibile, onnicomprensivo e antagonista, con quella, più terra terra, a un molteplice concreto, il più possibile accogliente, adatta ai bisogni del mondo globale e del suo azionista di riferimento, l'individuo di massa, sorta di «persona pubblica autonoma» che ha voglia di specchiarsi molto più che conoscersi. In termini editoriali ciò significa puntare su una scrittura come svago smart e consensuale, prodiga di informazioni e rassicurazioni identitarie [...]. Una scrittura che intrattenendoci ci istruisca su qualcosa di generico e ci faccia sentire migliori (se la letteratura non riesce a essere un piacere, che sia almeno un dovere civile e culturale cui non ci si è sottratti). (Simonetti 2018, 33)

La letteratura diviene rispecchiamento rassicurante su temi e idee condivise da autore e lettore, e che appaghino un vago e spesso sterile bisogno di engagement socio-politico. In questo quadro l'ambientazione nazista risponde a queste nuove esigenze, proprio in virtù della condivisa distinzione tra bene e male e perciò della rassicurazione morale che il lettore va cercando. La lotta collettiva - sia pure soltanto a parole - contro il male riconosciuto conduce alle «rassicurazioni identitarie», di cui parla Simonetti, e appaga il senso del «dovere culturale e civile». La letteratura degli anni Duemila ha questa cifra prioritaria.

D’altra parte il bisogno di storytelling resta vivo. Mentre il postmoderno aveva contaminato alto e basso, le narrazioni più recenti sembrano far percepire un nuovo distanziamento tra ciò che risponde al dovere culturale e civile e dunque parla di temi etici e ha un legame strettissimo con la realtà da una parte; e dall'altra ciò che è dichiaratamente solo intrattenimento e ricorre perciò ai più basici strumenti di storytelling che vengono accettati supinamente dal lettore.

È il caso dell'altro erede del romanzo di Dick, ovvero la serie TV (in generale e certamente con molte eccezioni, le serie TV, più volte all'intrattenimento disimpegnato, rappresentano narrazioni di questo secondo tipo) The Man in the High Castle. L'opera è dichiaratamente tratta dal romanzo di Dick e tuttavia è del tutto priva delle sue caratteristiche più complesse. Resta una spy story molto vicina al film di genere, in cui si oppongono un governo autoritario nazista e una resistenza che ha il compito di salvaguardare alcuni filmati vietati dal regime (simili al romanzo nel romanzo del precedente letterario). Protagonista è una donna tenace che ha la sfortuna di innamorarsi di un uomo della fazione opposta, forse pentito per amore. Si tratta insomma del più tradizionale dei plot, un po' 007 e un po' Liala in salsa storica. 
Dunque, mentre nel postmoderno lo storytelling insito nella struttura tematica dell'epoca nazista veniva utilizzato per rappresentare temi altri e letture sociologiche complesse, nell'epoca successiva il ricorso a questi temi ha una doppia finalità: quello di veicolare messaggi facili e condivisi; e quello di rappresentare lo sfondo per costruzioni narrative tradizionali.

\subsection{Esorcismi}

Gli eredi della Svastica sul sole rappresentano bene la nuova biforcazione, tipica degli anni Duemila tra letteratura (o in generale narrazione) alta e bassa, che in un'epoca precedente, ovvero durante il postmoderno fino a raggiungere il suo apice negli anni Novanta, convergevano. Di fronte al dilagare dello storytelling in ogni parte del sistema della comunicazione, da quella aziendale e pubblicitaria a quella giornalistica, la letteratura sembra ritirarsi nello spazio della realtà - persino oltre il realismo - come una forma di esorcismo nei confronti di quel sistema di narrazione logorato dall'abuso. Le storie vere tornano a popolare le librerie, sotto la spinta di autori di successo ma anche riconosciuti dalla critica.

Eppure gli esorcismi paiono inefficaci, o meglio apparenti. Mentre il lettore - e l'editoria che cerca di intercettarne e guidarne i gusti - pretende verità, depotenziando il ruolo di un'immaginazione che interpreta come frusta e abusata e di cui giocoforza diffida, il mercato risponde cercando le storie vere che possano avere all'interno una struttura romanzesca. Si determina uno iato tra la realtà raccontata e il fascino del racconto che gli autori più consapevoli non possono ignorare.

In Soldati di Salamina c'è un confronto tra il narratore e il personaggio eroico di Miralles che verte proprio su questa frattura. Nota il primo: «tutte le guerre abbondano di storie romanzesche, no?»; e il vecchio soldato, che porta nel corpo le ferite terribilmente reali di quelle guerre, risponde: «Solo per chi non le vive» (Cercas 2002, 198).

In Limonov, biografia dell'eponimo attivista e scrittore russo pubblicata nel 2011 da Emmanuel Carrère, si trova forse il caso più evidente del fascino dello storytelling in conflitto con la realtà vissuta (e pretesa dal lettore). Limonov appare infatti come una figura eroica, nel bene e nel male, che ha vissuto mille rocambolesche esperienze, dalla Russia archetipica della sua adolescenza, alla povertà estrema negli Stati Uniti, fino alle esperienze del carcere, della guerra in Serbia, dell'attività politica nel Paese natale. L'autore, borghese ricco, colto e di successo, si pone verso di lui quasi con una sorta di invidia o comunque di meraviglia proprio per la dimensione romanzesca di quella esistenza. Ma nel finale il contrasto tra questa immagine e la realtà dei fatti emerge in maniera bruciante: 
«È strano, però. Perché vuole scrivere un libro su di me?»

Sono colto di sorpresa ma rispondo, con sincerità: perché ha - 0 ha avuto, non ricordo più il tempo che ho usato - una vita appassionante. Una vita romanzesca, pericolosa, una vita che ha accettato il rischio di calarsi nella storia. (Carrère 2014, 353)

Di nuovo, è la dimensione «romanzesca» a colpire il narratore. Ma proprio questa dimensione è respinta nettamente dal protagonista della biografia:

E a questo punto Eduard dice qualcosa che mi lascia di sasso. Con la sua risatina brusca, senza guardarmi:

«Già, una vita di merda». (353)

L'intento principale di autori e lettori è quello di esorcizzare lo storytelling che fa perdere il contatto con la realtà. Ma come detto l'esorcismo è vano, inerte, perché comporta che si cerchino storie romanzesche e come tali vengono proposte: storie da storytelling, pronte all'uso, rassicuranti perché reali e quindi culturalmente accettabili e identificabili come 'alte', ma al tempo stesso succubi dei meccanismi ancestrali della narrazione.

Tra le molte storie che rispondono a queste caratteristiche, il mercato degli ultimi anni ha riconosciuto due grandi filoni che hanno anche il vantaggio tematico di essere entrambi di stretta attualità: la letteratura di migrazione che affonda su un tema sociale di indubbia importanza, e quella che si riferisce proprio al nazifascismo, un argomento che, come il precedente, rimanda a un chiaro impegno sociale. Mentre il primo filone richiederebbe uno studio a sé (cf. ad esempio Cinquegrani 2020), per il secondo gli esempi sono moltissimi. Emblematico, nei confini italiani, vedere i vincitori dei premi più popolari negli ultimi anni: La ragazza con la Leica di Helena Janeczek, Premio Strega 2018, ripercorre la vita e la morte della fotografa Gerda Taro durante l'ascesa del nazifascismo partendo proprio dalla natura eroica o non eroica della protagonista ( Da quando hai visto quella foto, ti incanti a guardarli. Sembrano felici, molto felici, e sono giovani, come si addice agli eroi. Belli non potresti dirlo ma neanche negarlo, e comunque non appaiono eroici per nulla», Janeczek 2017, 8); Le assaggiatrici di Rosella Postorino, Premio Campiello 2018, trasfigura narrativamente la storia reale di Margot Wölk incaricata di assaggiare il pasto di Hitler per scongiurare avvelenamenti; M. Il figlio del secolo di Antonio Scurati, Premio Strega 2019, è una biografia di Mussolini, che nella quarta di copertina ritorna sull'ambiguità tra romanzesco e realtà: «Sarebbe un personaggio da romanzo se non fosse l'uomo che più d'ogni altro ha marchiato a sangue il corpo dell'Italia» (Scurati 2018).

Sono solo alcuni esempi limitati all'Italia e ai casi macroscopici (e giocoforza parziali) dei più noti premi letterari, e tuttavia sono rap- 
presentativi di quanto questi argomenti possano attirare l'attenzione di pubblico e critica, proprio in virtù della contaminazione, in diversa misura, di realtà e finzione. Ma il caso più interessante, proprio per l'apparente intransigenza dell'operazione letteraria, è un altro romanzo di ampio successo internazionale, ovvero $H H h H$ di Laurent Binet Premio Goncourt 2010, che racconta del celebre attentato al famigerato Reinhard Heydrich avvenuto a Praga il 27 maggio 1942.

Il punto di partenza è proprio un esorcismo molto radicale rispetto all'invenzione letteraria. Si legge nella prima pagina:

Nel Libro del riso e dell'oblio Kundera lascia intendere che si vergogna un po' di dover dare un nome ai suoi personaggi, e benché quella vergogna non traspaia nei suoi romanzi, che pullulano di Tomas, di Tamina e di Tereza, la sua è l'intuizione di un'evidenza: c'è forse qualcosa di più volgare dell'attribuire arbitrariamente, per un puerile scrupolo di realismo o, nel migliore dei casi, per semplice comodità, un nome inventato a un personaggio inventato? Secondo me, Kundera avrebbe potuto spingersi oltre: c'è forse qualcosa di più volgare, infatti, in un personaggio inventato? (Binet 2011, 5)

L'invenzione letteraria appare dunque come una volgarità: è il punto di partenza di cui si è detto, l'esorcismo della letteratura alta verso la pura immaginazione che qui viene portata al suo parossismo. Nella lettura delle narrazioni storiche che l'hanno preceduto, Binet deve rilevare suo malgrado la resistenza, o addirittura la prevalenza, dell'immaginazione sulla storia:

Leggo anche molti romanzi storici, per vedere come se la cavano gli altri con le regole di questo genere letterario. Alcuni sanno dar prova di un estremo rigore, altri se ne fregano un po', altri ancora riescono ad aggirare abilmente gli ostacoli della verità storica pur senza affabulare troppo. Mi colpisce comunque il fatto che in tutti i casi l'immaginazione prevale sulla Storia. È logico, ma io stento a fare questa scelta. (21)

«Affabulare» è il compito di ciò che chiamiamo storytelling, e in questo passo risulta essere il nemico giurato dell'autore. Per lui deve prevalere la Storia e quindi è necessario aggirare il problema del romanzo storico, ovvero del realismo: «Il bello, con le storie vere, è che non devi preoccuparti del realismo» (Binet 2011, 28). Dove c'è realtà non serve realismo, ma dove si colloca esattamente il confine tra questi due livelli della conoscenza? Il dubbio irradia tutto il libro e ricompare spesso. Fino a dove può spingersi la descrizione del dettaglio che rende vividi i personaggi se non si può inventare nulla? Questa la domanda che innerva per esempio il capitolo 30 della prima parte: 
Natacha sfoglia distrattamente il numero del «Magazine littéraire» che mi ha gentilmente comprato. Si sofferma sulla recensione di un libro dedicato alla vita di Bach, il musicista. L'articolo si apre con la citazione dal libro: «C'è forse un biografo che non sogni di poter dire: Gesù di Nazareth aveva il tic di sollevare il sopracciglio sinistro quando rifletteva?» Mi legge la frase sorridendo.

Sul momento non ne valuto l'importanza e, fedele al mio vecchio disgusto per i romanzi realisti, mi dico: puah! Poi le chiedo di farmi vedere la rivista e rileggo la frase. Sono costretto a convenire che, in effetti, mi piacerebbe davvero disporre di quel genere di particolare su Heydrich. (Binet 2011, 40)

Il rigore col quale l'autore pretende di attenersi alla realtà depotenzia la forza del romanzo. Altrove deve ammettere di dover rinunciare quasi totalmente ai dialoghi, se non con funzione di «parabola» (24). Oppure quando parla di un altro romanzo, Like a Man di David Chacko, è costretto ad ammettere: «mi entusiasmo come guardando un western all'italiana». E aggiunge:

Quindi Chacko ha voluto anzitutto fare un romanzo, certo molto ben documentato, ma senza rimanere schiavo della documentazione. Prendere spunto da una storia vera, sfruttarne al massimo gli elementi romanzeschi, ma inventare tranquillamente quando ciò può essere utile alla narrazione senza dover rendere conto alla Storia. Un abile manipolatore. Un prestigiatore. Insomma, un romanziere. (193)

Ma, dato dunque questo modello negativo, questa idea negativa della manipolazione romanzesca, perché il libro funziona esattamente come un romanzo e in fondo contiene comunque quella dimensione di affabulazione che l'autore dichiara di voler evitare?

La risposta a questo interrogativo corrisponde al tema chiave di questo saggio. L'elemento romanzesco è insito nelle storie di cui si parla e per quanto si cerchi di sottrarre affabulazione e dunque storytelling, questo rientra perché è già nei fatti. Lo stesso Binet lo sa bene:

Dovetti aspettare due o tre anni per rendermi conto di ciò che avevo sempre sospettato: che per i suoi risvolti romanzeschi e la sua intensità quella storia superava i più improbabili racconti di immaginazione» (9)

Sono costretto a inchinarmi, ancora una volta, di fronte all'incommensurabile e nefasto potere della letteratura. Quel sogno, infatti, dimostra a chiare lettere che, con la sua indiscutibile dimensione romanzesca, Heydrich mi impressiona. (52; enfasi nell'originale) 
Questa impressione deriva non solo da quanto ogni episodio bellico potrebbe contenere di sinistramente affascinante, ma anche da una disposizione più definita e circoscritta che riguarda lo stesso protagonista nazista le cui conoscenze «si limitano in gran parte a quanto ricorda dei numerosi romanzi di spionaggio inglesi che divora da anni» (38), o comunque dai nazisti in generale:

Non bisogna credere a tutto quello che si racconta, specie se a raccontarlo sono dei nazisti: in genere, o scambiano per realtà i loro auspici e si sbagliano clamorosamente, come il grasso Göring, o mentono spudoratamente a scopo di propaganda, come Göbbels Trismegisto, che Joseph Roth chiamava «il megafono personificato». E spesso entrambe le cose. (186)

Il romanzo, dunque, con le sue scene di spionaggio, sparatorie, fughe, attentati, nasce naturalmente, è nei fatti, e anche l'autore alla fine lo deve riconoscere: «Credo di cominciare a capire: sto scrivendo un infraromanzo» (Binet 2011, 247).

Esiste dunque un romanzo che «sta sotto o sopra le righe del romanzo visibile», come definisce l'infraromanzo Giacomo Debenedetti in una lettera a Luigi Baldacci, ma resta comunque un romanzo, forse un romanzo al quadrato. È quasi impossibile, nonostante i ripetuti esorcismi, liberarsi dello storytelling, e benché il lettore sia opportunamente rassicurato sulla veridicità della storia, sul mancato cedimento alla sirene dell'affabulazione, sull'impegno sotteso a temi di certo moralmente irreprensibile, resterà pur sempre un'ampia e affascinante attrazione per il racconto: perché il nazismo è, non solo nella sua dimensione simbolica come dimostrava il postmoderno, ma anche nella sua dimensione storica, inscindibile dallo storytelling. 



\section{Il caso Vollmann}

Sommario 3.1 Introduzione. - 3.2 Europe Central: un romanzo massimalista. 3.3 Modo enciclopedico, lunghezza e intersemioticità. - 3.4 Esuberanza diegetica, coralità dissonante e onniscienza narratoriale. - 3.5 Compiutezza. - 3.6 Impegno etico.

Credo che la letteratura debba correggere la storia: la Storia è generalità mentre la letteratura è concretezza. La Storia è numero, la letteratura è individualità. [...] Quando dico correggere la Storia con la letteratura penso a questo: come offrire all'indifferenza della Storia il dono del concreto e del veritiero attraverso documenti autentici, lettere e oggetti che recano traccia di esseri reali. La letteratura è la concretizzazione dell'astratto della Storia. (Danilo Kiš, Homo poeticus. Saggi e interviste, 2009)

\subsection{Introduzione}

La memoria diretta degli eventi traumatici del Novecento, a causa della naturale scomparsa dei testimoni, sta lasciando il campo a una ricostruzione a posteriori e alla memoria d'archivio. La pretesa retorica di imbrigliare e rendere presente la Storia dal punto di vista della narrazione, di conseguenza, e il mandato etico che la memoria soggettiva veicolava - la memoria di chi aveva visto e vissuto - attraverso la grande quantità di scritture su quegli eventi, sono destinati ad incontrare un grande vuoto, a scontrarsi con la necessità di mantenere viva l'esperienza dell'evento senza che questo sia stato vissuto, e di ancorarlo al presente di una realtà la cui attestazione ontologica è da tempo entrata in crisi con il postmoderno (McHale 1987).

Il passato si presenta quindi nudo e irriducibile nella sua alterità, nella sua distanza, al cospetto del quale la scrittura, con le parole di 
Michel de Certeau, diventa una pratica che «mette in scena una popolazione di morti» ([1977] 2006, 117), «rito di sepoltura» (118) che «esorcizza la morte introducendola nel discorso» (118) (Piga Bruni 2018, 14). Di «pulsione negromantica» della scrittura ha parlato anche Mario Domenichelli $(2011,103)$ riferendosi a quel fenomeno che emerge e assume importanza come sintomo culturale nell'epoca della crisi delle grandi narrazioni (Lyotard 1981) e della post-historia (Vattimo 1985), quando storico e romanziere sono spinti dalla necessità di «evocare le voci dei morti» (Domenichelli 2011, 103) e dare la caccia all'«air du temps» (41) attraverso una ricerca documentaria e un particolare metodo di analisi e di scrittura (103). Le voci dei morti rappresentano, secondo questa accezione, quelle ammutolite dalla storia ufficiale, che riaffiorano nell'interpretazione postmoderna degli eventi passati sotto la facies di fantasmi che costituiscono una contro-narrazione, unico metodo e unica strategia possibile per interrogare la Storia intesa come fonte di conoscenza collettiva, nell'epoca che ne ha sfiduciato la condizione oggettiva. Lo scrittore negromantico si trova così alle prese con un complesso recupero della storia, e con differenti modalità di rappresentazione e interrogazione del passato (Piga Bruni 2018).

A proposito della problematica epistemologica relativa allo statuto della realtà e alla sua rappresentabilità, infatti, già Fredric Jameson aveva sostenuto che la produzione culturale postmoderna non fosse più nelle condizioni di interrogare direttamente il presunto mondo reale quanto, piuttosto, come nella caverna di Platone, dovesse tracciare «le nostre immagini mentali del passato sulle pareti tra cui è rinchiusa» (Jameson 2007, 42). Se di parvenza di realismo si tratta, insomma, nell'ottica della postmodernità ci si trova di fronte a un realismo

inteso come derivante dallo shock di aver compreso quello stato di reclusione, e di aver preso lentamente coscienza di una situazione storica nuova e originale, in cui siamo condannati a indagare la Storia per mezzo delle immagini e dei simulacri pop di quella storia, che come tale resta irraggiungibile per sempre. (42)

Risulta perciò centrale la messa a morte dell'evento nella postmodernità, quando i fatti lasciano il campo alle loro interpretazioni. Un processo cruciale che finisce con il problematizzare e ridiscutere un presupposto fondamentale del realismo occidentale: quello della contrapposizione tra il fatto in sé e la finzione (White 2006), che sfuma fino a sciogliere ogni possibile distinzione tra reale e immaginario. Sancendo l'imporsi ai danni della storia materiale della svolta linguistica, che a partire degli anni Settanta ha problematizzato la tradizione del realismo, è Hayden White ad inaugurare con il famoso 
Metahistory (1973) ${ }^{1}$ un'interpretazione della Storia intesa come costruzione sia retorica che narrativa, considerata come

un genere ibrido, prodotto di un'unione bizzarra, sebbene non innaturale, tra storia e poesia, (White 2006, 17)

mettendo in luce la stretta relazione che intercorre tra letteratura e ricostruzione storiografica (Piga Bruni 2018, 46).

Nel recente saggio La lotta e il negativo. Sul romanzo storico contemporaneo (2018) anche Emanuela Piga Bruni si è soffermata sulle conseguenze che questo processo ha comportato in termini narrativi, evidenziando il fatto che i romanzi storici contemporanei abbiano dato vita a una fervente sperimentazione:

Anche sulla scia della risonanza della Condition postmoderne di Lyotard (1979), il venire meno di una condizione oggettiva della Storia, della credenza di una possibilità di racconto coerente, spinge alla sperimentazione di diverse modalità di interrogazione sul passato. Questo si rivela soprattutto in quei romanzi che si misurano con eventi cruciali e traumatici della storia novecentesca. Gli approcci sono diversi, e la resistenza alla finzionalizzazione dei fatti reali del passato si esprime spesso nel trattamento poco mediato delle fonti, raccolte ed esibite nel testo sotto forma di inserti. $(2018,35)$

Nell'ambiguità che si è venuta a creare attorno allo statuto di verità assoluta Linda Hutcheon ha del resto intravisto una possibilità di emancipazione per lo scrittore, il quale, libero di proporre una narrazione critica della storia, può operare mettendo in luce la parzialità del punto di vista di chi racconta una vicenda. Prendendo le difese della produzione letteraria postmoderna, svincolandola dalle accuse di complicità nei confronti delle dinamiche tardocapitalistiche, Hutcheon (1988) ha parlato di una faglia apertasi tra romanzo storico tradizionale e postmodernismo, e ha teorizzato l'etichetta di historiographic metafiction. Si tratta di una definizione applicata alle forme di romanzo storico postmoderno, narrazioni che assottigliando il discrimine tra storiografia e racconto finzionale tendono a riscrivere il passato, sostituendo alla monoliticità della storia un corpus di narrazioni diverse:

by this [definition] I mean those well-known and popular novels which are both intensely self-reflexive and yet paradoxically also lay claim to historical events and personages. $(1988,5)$

1 Tradotto in Italia nel 1978 dall'editore Guida con il titolo Retorica e storia. 
E una lettura che viene per esempio ripresa da Angelo Petrella a proposito del romanzo $Q$ di Luther Blissett: quella del collettivo è infatti un'opera che mira evidentemente al recupero della memoria di eventi dimenticati, una contro-narrazione che intende rovesciare la narrazione egemone per affidare piuttosto la parola a soggetti subalterni, marginali, assumendone il punto di vista. In $Q$ infatti

la narrazione non si abbandona solo all'affabulazione, ma tende a riscrivere il passato in una nuova chiave, presentandosi come epica degli ex-centris, ovvero degli emarginati, dei reietti e degli sconfitti. (Petrella 2006, 146)

È sulla scorta degli studi di Hutcheon che Amy Elias in Sublime Desire. History and Post-1960s Fiction (2001) ha poi tentato una sintesi tra le diverse interpretazioni della storia ad opera del postmoderno: se l'historiographic metafiction è una categorizzazione che esprime 'una' delle prospettive con cui possiamo intendere il rapporto tra modernismo e postmodernità, in questo caso di cesura, per Elias invece risulta più utile ricollocare la narrativa storica postmoderna in una linea di continuità con la forma classica del romanzo storico, suggerendo che il rapporto con la storia nella finzione postmoderna è comunque debitore nei confronti di una tradizione classica tipica dell'estetica occidentale. Constatata quindi la vera o presunta «fine della storia» (Fukuyama 1992), agli scrittori della postmodernità rimarrebbe come unica possibilità la metahistory, cioè quella che Elias definisce:

the ability to theorize and ironically desire history rather than access it through discovery and reconstruction. (2001, XVII)

Il romanzo storico contemporaneo, a differenza dell'interpretazione della storia accessibile al soggetto per mezzo di uno studio empirico, o dell'alienazione modernista causata dal trauma provocato dall'evento storico, si fa invece portatore di una concezione della storia come di qualcosa che sfugge, che non si fa imbrigliare, un luogo di verità mai del tutto accessibile (Piga Bruni 2018, 53),

the realm of terror, of chaos, but also the realm of potential revelation. (Elias 2001, 55)

Come ha infatti sottolineato Piga Bruni sulla scorta di Elias, rifiutandosi di voltare le spalle alla storia il metahistorical romance si caratterizza piuttosto come narrazione problematica e politicizzata, che impegnata nella ricerca del mythos fondativo apre nuove strade per provare a stabilire una relazione con il passato, instaurando un rapporto con la materia storica improntato più al 'desiderio' che alla pretesa di una ricostruzione storiografica puntuale o a una controstoria: 
la coscienza post-traumatica di fine secolo si relaziona alla storia come un orizzonte desiderato ma mai raggiunto, che può essere soltanto avvicinato. Da qui un 'sublime storico' [...] che genera nuove rappresentazioni del passato storico. (Piga Bruni 2018, 53-4)

Abolito il tabù che riguarda la commistione tra fatto e fantasia (White 2006), alla letteratura viene quindi attribuito un primato nella ricerca di nuove forme di indagine che possano rappresentare l'evento storico e le figure che lo caratterizzano, libera di sperimentare e di dare vita a nuove forme narrative, come la metafiction storica, la 'biofiction', e così via, quelle forme cioè che

modificano l'idea stessa di realtà, presentando modelli di intersezione tra fatto e finzione che avrebbero un potere conoscitivo maggiore sia delle vecchie forme di realismo, sia del racconto storiografico che 'mette in intreccio' gli eventi stabilendo nessi causali dentro una forma chiusa. (Benvenuti 2012, 14)

Ancora, con Giuliana Benvenuti,

prima della 'svolta linguistica' e dell'analisi della scrittura storiografica come narrazione, potevamo distinguere un'opera storiografica da un testo letterario sulla base di una serie di convenzioni consolidate. Oggi le cose si sono notevolmente complicate, in primo luogo per la messa in discussione dei presupposti del 'realismo ingenuo', che investe tanto il discorso storiografico quanto quello romanzesco. $(2012,12)$

Rispetto ai grandi romanzieri storici dell'Ottocento quindi, da Balzac a Manzoni, da Stendhal a Tolstoj, le nuove forme di narrazione della storia aggirano il patto narrativo con il lettore, il cui compito di separare eventi reali e immaginari, realtà e finzione, si complica esponenzialmente. In molta narrativa contemporanea, al contrario, i due piani vengono sovrapposti e intrecciati, in alcuni casi ancora confusi nella modalità narrativa dell'‘autofiction', mentre in altri alla messa in scena dell'io autoriale si affianca una riflessione di tipo metanarrativo sul romanzo, di cui l'autore disseziona la genesi e i procedimenti (Piga Bruni 2018, 13-14). Confrontandosi con il problema della rappresentazione del dato storico, molti romanzi presentano cioè delle metanarrazioni focalizzate sul processo realizzativo e la complessità tecnica che questo comporta.

È il caso del romanzo HHhH. Il cervello di Himmler si chiama Heydrich (2011) di Laurent Binet, ${ }^{2}$ caratterizzato dalla presenza di

2 Vedi anche il capitolo «Postmoderno e Shoah» in questo volume. 
una massiccia ibridazione tra 'autofiction' e scrittura storiografica da parte dell'autore. Con $\mathrm{HHhH}$ Binet ricostruisce Operazione Antropoide, la missione pianificata dal governo ceco per assassinare Reinhard Heydrich, Reichsprotektor di Boemia e Moravia. Di pari passo con la narrazione attenta e minuziosa della storia dei due attentatori protagonisti, Jan Kubiš e Jozef Gabčík, Binet, fin dalle prime pagine, include se stesso nel piano diegetico della narrazione, facendosi coincidere con il personaggio che si occupa di ricostruire la storia:

quando arrivai a Bratislava, nel 1996, [...] una delle prime cose che domandai al segretario dell'addetto alla Difesa presso l'ambasciata [...] riguardava quella storia dell'attentato. $(2011,8)$

Ancora:

poco dopo il mio arrivo incontrai una bellissima ragazza slovacca di cui mi innamorai perdutamente e con la quale avrei vissuto un'appassionata storia d'amore destinata a durare quasi cinque anni. Fu lei a fornirmi qualche ulteriore elemento. (9)

E così via.

Nel corso della narrazione l'autore commenta diffusamente tramite inserti metanarrativi le ricerche condotte per scrivere la vicenda, analizzando documenti storici, film e romanzi sull'attentato; si tratta di passaggi che rettificano e correggono il testo, che, come un'opera non compiuta ma in divenire, mostrando errori e ripensamenti,

si sdoppia in due livelli, quello della storia ricostruita e quello della ricerca necessaria per ricostruire la storia. Il piano della riflessione accompagna lo sviluppo degli eventi come uno specchio, nel quale si riflettono commenti, digressioni e collegamenti ad altri libri. (Piga Bruni 2018, 98)

Così ad esempio l'autore sostiene dei fatti, come:

alle nove, finalmente, il primo carro armato tedesco entra in città, (Binet 2011, 97)

che poi finisce per ritrattare:

in realtà, non so se sia stato un carro armato a entrare per primo a Praga. (97)

Oppure, ancora: 
be', no, non è così, sarebbe troppo semplice. Rileggendo uno dei libri fondamentali della mia documentazione [...] mi accorgo con sgomento degli errori che ho commesso parlando di Gabčík. (110)

In alcuni passi poi Binet si sbaglia:

quando [Heydrich] viene a sapere che il capo del Secret Intelligence Service inglese si fa chiamare M (sì, come in James Bond), decide sobriamente di farsi chiamare $\mathrm{H}$, (39)

per poi correggersi a distanza di poche pagine:

ho detto una sciocchezza, vittima di uno scherzo della memoria nonché di un'immaginazione un po' invadente. In realtà, il capo dei servizi segreti inglesi, a quell'epoca, si faceva chiamare C, e non $\mathrm{M}$ come in James Bond. Anche Heydrich si è fatto chiamare C, e non H. (44)

Tuttavia, quello che viene messo in luce in $\mathrm{HHhH}$ è soprattutto lo statuto ambiguo di una narrazione che si dichiara e prova ad essere il più possibile aderente ai fatti e agli eventi storici - «dico che inventare un personaggio per comprendere dei fatti storici è come manipolare le prove» (234) - ma che non riesce tuttavia ad esimersi completamente dall'affabulazione narrativa:

questa scena è perfettamente credibile e totalmente immaginaria, come quella precedente. Che impudenza trasformare in una marionetta un uomo morto da tempo, che non può difendersi. Fargli bere del tè mentre probabilmente gli piaceva solo il caffè. Fargli indossare due cappotti mentre forse ne possedeva solo uno. Fargli prendere l'autobus mentre può aver preso il treno. Decidere che è partito di sera e non di mattina. Mi vergogno. (109)

Addirittura, secondo Binet anche una sola eventuale concessione finzionale alla ricostruzione dell'evento storico potrebbe mettere in crisi l'intera opera:

mi chiedo come faccia Jonathan Littell a sapere che Blobel [...] aveva una Opel. Se davvero Blobel viaggiava in Opel, tanto di cappello. Ma se è un bluff, mette in crisi l'intera opera. È vero che i nazisti si rifornivano massicciamente da Opel, e quindi è del tutto verosimile che Blobel possedesse un veicolo di quella marca. Ma verosimile non equivale ad accertato. (232) 


\subsection{Europe Central: un romanzo massimalista}

Europe Central (2010) di William T. Vollmann si colloca all'interno di questo complesso ed eterogeneo panorama definito dall'ambivalente rapporto tra memoria e narrazione, costituito da un lato dalle nuove possibilità di rilettura della storia che si sono dischiuse alla letteratura, e dall'altro dall'oscillazione degli autori tra libertà finzionale e ricerca d'archivio, tra puro artificio letterario e documento storico. Il romanzo di Vollmann costituisce un ambizioso tentativo letterario di ricostruzione degli eventi epocali del Novecento, raccolti all'interno del quadro geografico europeo: il cuore pulsante dell'Occidente del XX secolo, un palcoscenico simbolico sfruttato per un'operazione orientata alla narrazione e al recupero degli eventi traumatici tra la fine della Prima guerra mondiale e la caduta del muro di Berlino. La complessa e febbrile operazione di scrittura di Vollmann, che si sposta costantemente tra Est ed Ovest, dalla Repubblica di Weimar al muro di Berlino, dall’Unione Sovietica alla Germania nazista attraverso il fronte orientale della Seconda guerra mondiale, intreccia con incedere epico decine di storie drammatiche ed epocali, componendo una serie di racconti che con una fitta trama di rimandi e protagonisti ricorrenti si avvicendano lungo le oltre mille pagine del romanzo. Nello sfaccettato vortice di personaggi che si muovono all'interno dell'Europa in rovina, gli stessi Hitler e Stalin, il compositore Šostakovič a Leningrado, i generali dell'esercito tedesco e sovietico Paulus e Vlasov sul fronte russo, l'Obersturmführer Gerstein, la poetessa Achmatova e la leggendaria partigiana Zoja, sono solo alcune delle figure che animano l'affresco epocale ritratto da Vollmann.

A differenza di un approccio alla vicenda storica à la Binet, Europe Central si caratterizza per una forma di commistione in cui la distinzione tra reale e immaginario risulta apparentemente sospesa e indecifrabile: Vollmann mescola cioè minuziose ricerche bibliografiche a scene potenti, del tutto inventate, tramite una scrittura che annulla completamente la distinzione tra questi due piani. L'autore propone infatti la narrazione degli eventi storici più importanti del XX secolo, collocandoli alla stessa altezza di quelli di invenzione e facendoli risultare inestricabili tra loro nel corso della narrazione, salvo poi recuperarli e scioglierli in due passaggi chiave posti oltre la fine del romanzo. Il primo è contenuto nell'appendice «Un triangolo amoroso immaginario: Šostakovič, Karmen, Konstantinovskaja», in cui Vollmann si sofferma sulla vicenda dei tre personaggi e scrive che «per finalità narrative» ha inventato «molto delle interrelazioni attribuite a questi tre individui» $(2010,1057)$. Il secondo è dato dall'excursus bibliografico, un apparato di oltre 700 note al testo di decine di pagine in cui l'autore riprende e svela dettagliatamente, a tratti in maniera maniacale - non senza ironia Vollmann riporta più volte le spese sostenute personalmente per le traduzioni dei documenti dal 
russo all'inglese -, le fonti di tutti i passaggi storicamente documentati utilizzati nel romanzo, scrivendo che

I personaggi che compaiono in questo libro sono, in gran parte, realmente esistiti. Ho svolto ricerche sulle loro biografie con tutta la cura di cui sono capace, ma la mia resta pur sempre un'opera di narrativa [...] Mi scuso per eventuali, involontarie offese arrecate ai vivi, ma ripeto: questa è un'opera di narrativa. (963)

Nonostante quindi la mole bibliografica di documenti e di fonti storiche costituisca il materiale di riferimento di tutta la narrazione - e in qualche modo modelli la struttura stessa di Europe Central, dai dialoghi dei protagonisti fino alle scene rappresentate -, Vollmann ribadisce a più riprese l'importanza della natura finzionale dell'opera, rivendicando la propria libertà di scrivere di finzione anche e nonostante la scelta di narrare fatti che lui stesso attesta storicamente tramite il massiccio uso di fonti bibliografiche. Perché in fondo, secondo Vollmann,

Tutto ciò è comunque il frutto delle più labili speculazioni, privilegio che Dio concede agli autori di narrativa. (1007)

Si tratta perciò di un uso della narrazione fortemente motivato e ricercato dall'autore, come vedremo, una narrazione che viene impiegata per riscattare, seppur all'interno di traiettorie esistenziali già inscritte nella storia, le vicende dei suoi personaggi:

Il matrimonio [di Šostakovič] con Nina Varzar fu infelice per molti aspetti, e ho voluto concedergli, almeno nella finzione, un grande amore... che lui può aver benissimo provato per la sua ultima moglie, Irina. A lei e ai suoi figli - poiché in Europe Central la passione di Šostakovič per Elena domina la vita del compositore fino alla fine, inclusi gli anni dell'ultimo matrimonio - chiedo perdono per certe distorsioni della realtà imposte dagli obiettivi di questo libro. (1058)

L'intenzione di valorizzare la vita delle persone travolte dalla storia, con lo scopo di

scrivere una serie di parabole su alcuni famosi, famigerati o anonimi attori morali europei osservati nei momenti di importanti decisioni, (963)

ha sicuramente a che fare con gli «obiettivi di questo libro» di cui parla Vollmann. Inoltre, se da un lato la narrazione delle vicende di uomini famosi o anonimi testimonia l'intento 'negromantico' di Voll- 
mann - che ricostruisce documenti alla mano le vicende di comparse e personaggi storici di primo piano, e inventa quelle di personaggi fittizi che gli gravitano intorno -, dall'altro riecheggia anche il preciso modello di riferimento di Europe Central: lo scrittore Danilo Kiš, omaggiato dallo stesso Vollmann in apertura di romanzo:

In memoria di Danilo Kiš, il cui capolavoro 'Una tomba per Boris Davidovič' mi ha tenuto compagnia per molti anni, mentre mi preparavo a scrivere questo libro.

La citazione non è ovviamente fine a se stessa, avendo avuto le posizioni di Kiš sul rapporto tra letteratura e storia un evidente ascendente sulla realizzazione di Europe Central. Così sostiene ad esempio l'autore serbo in Homo poeticus (Kiš 2009), dove sembrerebbe di cogliere una spia del 'modus operandi' di Vollmann:

Si è verificato un cambiamento sostanziale nella letteratura del $\mathrm{XX}$ secolo, che non è né può più essere romantica nel senso storico del termine romanticismo. Nel romanticismo l'immaginazione era la forza motrice della letteratura. Con l'eredità che ci ha lasciato la storia del nostro secolo è chiaro che l'immaginazione, e con essa il romanticismo, hanno perso ogni significato. La storia moderna ha plasmato aspetti così autentici della realtà che allo scrittore odierno non rimane altro se non dare loro una forma artistica o, se serve, «immaginarli», ossia usare i dati autentici come materiale grezzo e fornire loro una nuova forma tramite l'immaginazione. Certo, può essere utile partendo dalla realtà inserire documenti storici nella narrazione come prova dell'autenticità di quanto è stato scritto. D’altra parte un documento storico può servire anche alla mistificazione: attraverso il documento si suggerisce al lettore l'autenticità, il documento suggerisce al lettore di credere all'autenticità di tutto ciò che legge, mentre l'autore persegue obiettivi diversi. Per questo è importante per un bravo scrittore «confondere» le proprie idee, ossia inventare un «documento storico» - ma non la storia - o, se vuole, «falsificarlo» per identificare di nuovo, attraverso l'immaginazione, la realtà storica. $(2009,289)$

Tuttavia, oltre a rappresentare un rimando alla poetica di Kiš, l'obiettivo di Vollmann di dare voce e in qualche modo giustizia ai personaggi di Europe Central riguarda anche e soprattutto l'impegno etico che caratterizza la sua prosa, come vedremo; ed è proprio a causa della presenza di questo aspetto - a cui vanno sommati la lunghezza del romanzo, il suo polifonico tentativo di rappresentare la Storia e altri elementi cruciali che verranno ora analizzati - che in questo intervento viene avanzata l'ipotesi di classificare Europe Central come solido esempio di quel tipo di opera letteraria che Stefano Erco- 
lino ha denominato 'romanzo massimalista', etichetta che dà anche il nome al suo fortunato saggio del 2015.

Ercolino ha proposto la definizione di 'romanzo massimalista' per quel genere di romanzo ibrido sviluppatosi nella seconda metà del Novecento negli Stati Uniti, «nato al crocevia tra l'estetica modernista e quella postmoderna» $(2015,9)$. Definito 'massimalista' «per la multiforme tensione massimizzante e ipertrofica delle narrazioni» (9), Ercolino ha elaborato questa definizione esaminando una rosa di opere letterarie piuttosto eterogenee tra loro - Pynchon, L'arcobaleno della gravità; Foster Wallace, Infinite Jest; DeLillo, Underworld; Smith, Denti bianchi; Franzen, Le correzioni; Bolaño, 2666; Babette Factory, 2005 dopo Cristo -, romanzi che secondo l'ipotesi avanzata nel saggio condividerebbero una serie di caratteristiche ben precise: lunghezza, modo enciclopedico, coralità dissonante, esuberanza diegetica, compiutezza, onniscienza narratoriale, immaginazione paranoica, intersemioticità, impegno etico, realismo ibrido. Le categorie che caratterizzano e definiscono il romanzo massimalista secondo Ercolino rappresentano pertanto degli strumenti utili per provare a scomporre la complessità e a decifrare i significati di Europe Central, romanzo che, come vedremo, sembra racchiudere la maggior parte delle caratteristiche individuate ne Il romanzo massimalista. La seconda parte di questo intervento si concentrerà perciò sull'analisi del romanzo di Vollmann alla luce della griglia strumentale dedotta dallo studio di Ercolino, analizzando l'efficacia della sua funzione strutturale all'interno della narrazione di Europe Central.

\subsection{Modo enciclopedico, lunghezza e intersemioticità}

Definito da Ercolino come:

particolare atteggiamento estetico e conoscitivo consistente in una tensione narrativa totalizzante diretta alla rappresentazione sintetica di realtà e saperi eterogenei, $(2015,70)$

il 'modo enciclopedico' è lo strumento - e non il fine, come sostenuto invece da Stephen Burn (2007) - utilizzato da Vollmann per creare una «narrazione sintetica della totalità del reale» (Ercolino 2015, 59). In Europe Central Vollmann affronta lungo una linea cronologica progressiva - dal 1914 al 1975 - più di mezzo secolo di storia europea, delineando uno spaccato delle dittature nazista e stalinista e rappresentando svariate realtà sociali attraverso continui e sistematici riferimenti al mondo dell'arte (Käthe Kollwitz), della poesia (Anna Achmatova), della musica (Dmitrij Šostakovič) e della cinematografia (Roman Karmen): come per gli autori massimalisti presi in 
esame da Ercolino, anche per Vollmann sembra che il centro dell'interesse enciclopedico sia rappresentato dallo «scibile umano nella sua totalità» (Ercolino 2014, 79).

Se, come scrive Ercolino, un romanzo che

aspira a misurarsi con il mondo intero non può farlo se non assumendone l’ampiezza, $(2014,46)$

non si può dire che questo non valga anche per Europe Central. L'edizione italiana infatti è lunga 1063 pagine, che a fatica contengono l'esuberanza narrativa di Vollmann, impegnato a tracciare le coordinate storiche ed esistenziali dei numerosi personaggi che compaiono nel romanzo. La lunghezza di Europe Central rappresenta una 'possibilità', legata da un lato alla cifra sperimentale del romanzo, dall'altro alla sua «ambizione totalizzante di matrice epica» (Ercolino 2014, 43): l'epica come caratteristica strutturale quindi, come

forma incline alle digressioni: abbonda di episodi che si collocano a lato dell'Azione fondamentale. (Moretti 1994, 44)

La lunghezza di Europe Central garantisce in questo modo la proliferazione enciclopedica e polifonica di tutte le storie che lo compongono, «base materiale imprescindibile sulla quale poggia il progetto narrativo massimalista» (Ercolino 2014, 46): è la lunghezza infatti a permettere il dispiegarsi di tutte quelle strategie - enciclopedismo, coralità, digressioni, moltiplicazione dei filoni narrativi - che creano quell'effetto mondo' di cui ha parlato Franco Moretti, che trasmette al lettore l'impressione

di trovarsi al cospetto del mondo. Che rendano cioè il testo simile al mondo: aperto, eterogeneo, incompleto. (Moretti 1994, 55)

Secondo Ercolino l'enciclopedismo rappresenta il desiderio di afferrare e dominare il mondo, aspirazione che

affonda le radici nell'antichissima ambizione sintetica e totalizzante dell'epica, a partire proprio da Omero ed Esiodo. Un desiderio che la scienza non potrà mai soddisfare del tutto per via della sua strutturale e sempre crescente settorialità. (Ercolino 2014, 60)

E in effetti quello dell'enciclopedismo è una modalità tutta finzionale che permette a Vollmann di fare i conti con la Storia, in qualche modo - e nonostante l'uso massiccio di materiale bibliografico - superando la necessità di utilizzare il documento storico che da solo non riuscirebbe a colmare la lacuna alla base della trasmissione della memoria dei personaggi di cui racconta le vicende, memoria in so- 
speso tra il dramma del tempo passato e la lontananza del destinatario del tempo presente. Così Vollmann nel corso della narrazione si riferisce spesso e in maniera ironica alla 'Grande enciclopedia sovietica' come allegoria del documento storico, statico e monumentale, che si rivela spesso una fonte insufficiente ai suoi scopi, arida, e di cui rivela soprattutto la tendenziosa e potenziale manipolabilità:

Achmatova era l'autrice di Requiem, poesia ammirata persino dal nostro obbediente Šostakovič [...]. Inutile sottolineare che non si è guadagnata neppure una menzione nella Grande enciclopedia sovietica. (Vollmann 2010, 199)

\section{Ancora:}

La fonte più infallibile riguardo a questo periodo è, naturalmente, la nostra Grande enciclopedia sovietica che recita: 'Negli anni Trenta, la cultura musicale sovietica fece notevoli progressi. La sua ristrutturazione era, nella sostanza, compiuta'. Eppure Šostakovič continua a non capire di doversi ristrutturare. (199-200)

Il modo enciclopedico di Europe Central, romanzo della «moltiplicazione» e della «differenziazione dei tentativi enciclopedici» (Ercolino 2014, 59), oltre a riprodurre

una sintesi parziale, una sineddoche, riguardante un segmento specifico di un certo campo del sapere, (73)

diventa soprattutto un trait d'union tra il dramma del personaggio di cui Vollmann narra le vicende e il contesto storico in cui queste hanno luogo. Nel caso del capitolo «Donna con bambino morto», ad esempio, i riferimenti all'arte figurativa creano un ponte tra la pittrice e scultrice Käthe Kollwitz e la Storia, trasmettendo in maniera drammaticamente vivida al lettore la vicenda che Vollmann descrive. Sullo sfondo dei tumulti della Berlino degli anni Venti, tra le violenze dei Freikorps e i prodromi dell'ascesa al potere del nazismo, in «Donna con bambino morto» Vollmann racconta la storia dell'artista, alle prese con la propria creatività e l'elaborazione del lutto per la perdita in guerra del figlio Peter. Gli affondi in campo artistico diventano un tutt'uno con la narrazione della Storia, che incombe sempre sullo sfondo e nella quale si amalgama la storia dell'artista:

La leggenda secondo cui sarebbe stata la morte del figlio a ispirare quest'opera è facilmente confutabile. Morte, donna e bambino, ad esempio, fu eseguito nel 1910, quattro anni prima della scomparsa di Peter. Sul piano formale, assomiglia allo schizzo con il gessetto dell'anno precedente, intitolato Addio: il viso del bambi- 
no, delizioso, bianchissimo e realistico, stretto contro il viso più ampio e più grigio della madre che, nel suo cordoglio, sembra dissolversi nel nerissimo sfondo informe. Nel 1903, tanto nella Pietà quanto in Madre con bambino morto, le posizioni erano invertite: la madre che stringe il bambino da una posizione sovrastante, con la testa reclinata sul petto, mentre la testa del bambino penzola nel vuoto, le labbra leggermente schiusa sulla faccia bianca. C'era stata un'altra Madre con bambino morto in quello stesso anno, quasi alla maniera di Blake, con quel protendersi in primo piano della gamba e del piede: la madre è seduta a gambe incrociate, con un ginocchio sollevato, la testa china sul bambino, la cui forma ammantata e vagamente fallica finisce per fondersi con quella materna; di lei si vedono l'orecchio, la fronte corrugata e un occhio infossato, ma solo in quella forma sfumata e decomposta che accomuna gli embrioni e le opere d'arte incompiute. (Vollmann 2010, 63-4)

\section{Si legge ancora che Käthe Kollwitz lavorava}

senza consultarsi con nessuno al fiero protocubismo di quegli anni, in cui il passato figurativo e classico era morto quanto il Secondo Reich: morto, morto! Morto come gli ufficiali zaristi che erano affogati nei loro campi da parata fangosi e infestati di erbacce, affinché il partito di Lenin e Stalin potesse marciare sulle loro facce putrescenti. Sin dal 1912 lei aveva una stanza tutta per sé in Siegmundhof, dove si dedicava alle arti plastiche [...]. Erano gli anni in cui le figure, nei dipinti degli altri, cominciavano a diventare sempre più piatte, vistose, distorte, con colori che per lei erano addirittura dolorosi, sebbene le piacessero alcuni dei calligrafici cavalieri al galoppo di Kandinskij. Le disperate e feroci caricature di Grosz, l'amarezza radiografica di Otto Dix, per non parlare del costruttivismo astratto: lei non nuotò mai in quelle acque. Käthe Kollwitz continuò a ritrarre povera gente, gente affamata (figure bianche su sfondo scuro, gesso scuro su carta Ingres bruna), donne violentate, madri con bambini morenti, madri con bambini morti. In fin dei conti, dipingeva soprattutto se stessa, il proprio volto scimmiesco terrorizzato, che pensava e soffriva. Anche lei era una madre con bambino morto. (59-60)

L'excursus tra le forme di conoscenza e saperi più rappresentativo dell'enciclopedismo di Europe Central è senz'altro quello realizzato attorno alla figura del geniale compositore russo Šostakovič. Nel capitolo «Opus 40» Vollmann sintetizza la relazione erotica del musicista con l'amata Elena Konstantinovskaja e la nascita dell'Opus 40, e la musica funge da vera e propria struttura portante per l'evolversi della narrazione: 
Elena, You're the one for me, l'unica donna che desidero, le disse. [...] Non l'aveva ancora baciata, e le sue ghiandole dei bassi e degli alti avevano già cominciato a comporre l'Opus 40, anticipazione della più bella delle fughe di Šostakovič. (114)

La realizzazione del celebre componimento viene quindi letta alla luce della relazione tra Šostakovič ed Elena:

Lei rise di gioia e gli saltò addosso; questa fu la genesi del quarto movimento (un altro Allegro); la si consideri una vivace eppur maestosa danza in una tonalità minore, una danza non di scheletri - che sono troppo maliziosi, troppo drammatici - sebbene l'Opus 40 cada talvolta in quello che diventerà il caratteristico grigiore di Šostakovič. Il pianoforte la riporta alla vita: Elena e Šostakovič stanno rincorrendosi a vicenda come gatti. Un rinomato pianista che ha eseguito questa composizione sostiene che 'il brio qui ha un senso sinistro più che esibizionistico', ma io dissento: Šostakovič è felice! Ecco che arriva il pizzicato: Elena sta facendo scorrere delicatamente e con affetto le sue lunghe unghie sulla pancia di lui. Dopo di che il pianoforte precipita in gioiosa cascata dentro un tiepido letto di strumenti a corde, in cui riluce lo scintillante, concitato ed esperto amoreggiare della coppia. [...] Si torna alla canzone di apertura, a quella sontuosa aria russa, che si stiracchia in diverse variazioni post coitum, finché l'Opus 40 termina con una deliziosa sorpresa di denti che scattano: di nuovo, il morso di Elena, che lascia su di lui un tenero marchio di proprietà, proprio lì sul lato del collo. (123)

Nel capitolo «La palma di Deborah» è ancora attraverso la «peculiare dimensione estetica e simbolica» (53) enciclopedica dell'Ottava sinfonia di Šostakovič che Vollmann descrive l'invasione tedesca della Polonia occupata dall’Unione Sovietica - e allo stesso modo Vollmann rileggerà poi l'assedio di Leningrado tramite la composizione della Settima sinfonia:

E giunse così la notte tra il 21 e il 22 giugno 1941, quando la severa e contagiosa melanconia nell'ouverture dell'Ottava sinfonia si tramuta stridendo in esplicito allarme. Dopo un tetro strimpellare di corde, si leva ancora più in alto sempre più stridula, ma adesso con l'aggiunta di una componente marziale. Colpi di tamburo simili a distanti raffiche di mitragliatrici annunciano la guerra vera e propria, e i corni strillano come le sirene degli allarmi aerei. 'Barbarossa' comincia: dieci contrabbassi, dodici violoncelli, dodici viole, trenta violini di due tipi diversi, quattro trombe, quattro flauti, due oboi, un corno inglese, due clarinetti, uno basso e uno piccolo, e altri ventidue strumenti disposti su un fronte 
lungo quattromilacinquecento chilometri. [...] La Russia si sveglia troppo presto in quel nero mattino, lacerandosi nell'urgenza di ottoni fatta di una molteplicità di paure solitarie [...]. La sinfonia prosegue gemendo. Non una nota, bensì zaffate di tetraggine e orrore. Una fanfara di un'idiozia terrificante annuncia la testa di sbarco del nemico: hanno conquistato Riga [...]. Il secondo movimento, nel quale, secondo un critico, 'qualsiasi accenno di allegria è rapidamente schiacciato e trasformato in ironia e causticità', potrebbe quasi essere musica di sottofondo per film muti [...]. Il terzo movimento, l'allegro ma non troppo, comincia in volo, e lo spartito, il pallido e piatto foglio delle sconfinate steppe ucraine, è in parte oscurato da campi e città in fiamme la cui distruzione è stata tradotta in musica con archi gravi. [...] I corni annunciano che 'loro sono qui' e scavalcano strisciando una bassa cresta dorata con le loro armi puntate contro di noi. Scappiamo, scappiamo, scappiamo! (233-5)

L'enciclopedismo di Europe Central è strettamente correlato ad un'altra delle caratteristiche del romanzo massimalista, con la quale crea un'interazione da cui l'immaginario 'ibrido' di Europe Central ricava dei «potenti strumenti espressivi e temi forti» (Ercolino 2014, 201). Si tratta dell' 'intersemioticità': Ercolino scrive che

l'immaginario letterario contemporaneo sembra poggiare saldamente e a diversi livelli sull'interscambio semiotico; un immaginario polimorfico, proteso verso il superamento di secolari tabù estetici. Il dialogo sempre più serrato in atto fra i diversi media arricchisce e rigenera i singoli linguaggi artistici all'insegna dell' $i$ bridazione. $(2014,200)$

In «Ampio è il mio paese natio», il capitolo dedicato al cinema documentario di Roman Karmen, Vollmann mette in relazione le tecniche artistiche del regista con il suo rapporto fittizio con Elena Konstantinovskaja, che compare ancora come musa ispiratrice: un'interazione complessa che

si presenta nel segno di 1) un amalgama fra reale e immaginario, o 2) della pura finzione, (Ercolino 2014, 205)

dove la cinematografia di Karmen diventa

un potente dispositivo semantico in grado di contribuire in modo determinante alla produzione dell'intreccio e di influenzare le tecniche compositive. (206)

Vollmann allora scrive: 
E veniamo ora alla Spagna: c'è, nei suoi cinegiornali, una sequenza di una forza sconvolgente che mostra un'anziana donna in nero con il suo bambino, anche lui vestito di nero, tenuto stretto al grembo come fosse uscito da un'opera di K. Kollwitz; il bambino guarda altrove, la donna fissa dalla nostra parte con un'espressione spaventata; quindi, un ragazzo rivolge a noi lo sguardo da lontano; più vicino, una giovane donna è seduta di traverso su una coperta e ci fissa con un'aria imperscrutabile; sono tutti seduti a terra; e in primo piano c'è una figura distesa su un fianco, che ci volge le spalle e protende le mani bianche verso gli altri; ha un fagotto sotto la testa, e a una prima occhiata non si capisce se questo essere sia vivo o morto. [...] Queste persone sono profughi; i bombardieri fascisti stanno arrivando. E qui Karmen trova il modo di rendere giustizia da un punto di vista artistico sia ai soggetti sia al proprio cuore. Perché ora? Forse perché è innamorato. È proprio in questo snodo della sua carriera che notiamo come Karmen passi gradualmente dalle riprese di gruppi a quelle di singoli individui. Di nuovo, mi domando perché; e mi viene in mente la stessa risposta: i sentimenti che nutriva per Elena furono tali da fargli comprendere fin nel profondo del cuore come ciascuno di noi possa in un certo senso rappresentarci tutti, così come il tutto contiene il singolo elemento. $(2010,302-3)$

\subsection{Esuberanza diegetica, coralità dissonante e onniscienza narratoriale}

Vollmann è noto soprattutto per essere non solo uno scrittore prolifico, ma una sorta di vero e proprio grafomane, un autore dotato di una «maximalist prolificacy» che rende i suoi romanzi e i suoi saggi «not an oeuvre but an archive» (Liebtag 2015, 197). In effetti, come è stato appena sottolineato, in Europe Central i linguaggi, i saperi e le voci dei personaggi si accumulano in maniera parossistica, «determinando una complessità e una ricchezza dialogiche probabilmente senza precedenti» (Ercolino 2014, 103). È possibile individuare le basi di questa ricchezza tanto nella lunghezza del romanzo quanto nelle forme di enciclopedismo appena analizzate, per cui la

rappresentazione di una gamma molto ampia di saperi e di linguaggi contribuisce alla costruzione di un impianto narrativo polifonico, conforme alla eterogeneità e alla multidisciplinarietà di un'enciclopedia. (Ercolino 2014, 103)

La narrazione del romanzo è quindi «ipertrofica e ultradensa», e le storie e i personaggi di Europe Central sono numerose, "come innumerevoli sono le digressioni e i temi affrontati» $(2014,121)$ : 
Il romanzo massimalista abbonda di materiale diegetico [...]. Ci si trova davanti a [...] un eccesso discorsivo che fa apparire un romanzo massimalista come un fiume in piena, il cui materiale narrativo sembra proliferare quasi spontaneamente [...]. La parola d'ordine è inclusione, non lasciare niente fuori, far entrare il mondo intero nel romanzo [...]. [Il romanzo massimalista ha] la tendenza a inglobare una gamma molto estesa ed eterogenea di materiali narrativi nella diegesi, quasi appunto a voler 'consumare ogni cosa con le parole'. (122-3)

Secondo Ercolino solo una scrittura di tipo frammentario può prestarsi all'inserzione di materiali così diversificati, consentendo grande libertà nella loro disposizione: da un punto di vista formale, infatti, alla base di questa coralità di voci e di saperi che «non consente l'affermarsi di un'istanza narrativa dominante» $(2014,87)$ l'autore ricorre all'uso del 'frammento' quale strumento compositivo privilegiato: perché la narrazione del romanzo non si presenta come un insieme compatto, ma procede per unità diegetiche indipendenti, frammentarie. Europe Central infatti si suddivide grossomodo in cinque parti: una di apertura («Acciaio in movimento»); una prima parte focalizzata sugli eventi tra la Prima guerra mondiale e l'invasione tedesca della Polonia; una centrale che sviluppa in un doppio movimento che procede da est verso ovest le dinamiche della Seconda guerra mondiale; una dedicata agli eventi successivi alla guerra e una di appendice extradiegetica («Fonti» e «Un triangolo amoroso: Šostakovič, Karmen, Konstantinovskaja»).

Ognuna di queste parti è formata da diversi frammenti/capitoli, in cui lo spazio tipografico «è sempre il segnale di un cambiamento di scena» (Ercolino 2014, 90), a cui è riconducibile una variazione del punto di vista o uno spostamento nello spazio e nel tempo, dove

il passaggio da un frammento all'altro è assimilabile a un'ellissi fra scene che scompone il racconto in quadri familiari narrativamente autonomi, ma collocabili all'interno di un'unica progressione narrativa. (94)

In Europe Central è notevole il fatto che i narratori cambino spesso da un capitolo all'altro, come delle vere e proprie personificazioni del contesto storico-politico in cui si svolge la vicenda narrata. Del resto lo spostamento del punto di vista è un tratto piuttosto evidente e caratteristico del romanzo, considerando che spesso questo movimento avviene addirittura anche all'interno dello stesso frammento: nel capitolo «La palma di Deborah», ad esempio, la narrazione di Vollmann passa da un 'noi' intradiegetico sovietico, che racconta la fuga caotica all'arrivo delle truppe tedesche, per poi compiere un doppio movimento scivolando bruscamente ad un 'noi' nazista e tornando infine alla prospettiva sovietica di partenza: 
Nascondiamoci! Arrivano. Noi fuggiamo. Arrivano! In modo assai improvviso, noi siamo loro, e tutto diventa allegro, come il ghigno di un cadavere. Noi nazisti continuiamo ad avanzare sparando [...]. Scavalcando la stessa bassa cresta da dietro la quale scrutavamo, in quell'epoca primordiale in cui noi eravamo noi, ora osserviamo i Rossi che fuggono verso l'orizzonte. Non importa: i nostri aerei da caccia li stermineranno quasi tutti [...]. Ora la musica ha un nuovo scarto [...], e di nuovo noi siamo noi, che corriamo e corriamo inseguiti dai latrati dei corni d'ottone. (Vollmann 2010, 236)

Questo rapido avvicendarsi del narratore interno avviene, in maniera più diluita, anche in "Sortita», capitolo dedicato alla tragica figura del generale sovietico Andrej Andreevič Vlasov che da eroe della resistenza antinazista si trasforma nell'odiato disertore al servizio del Reich. Fintanto che Vlasov si trova al comando della II Armata d'assalto incaricata di spezzare l'assedio di Leningrado, il narratore è evidentemente un sovietico: lo si può dedurre dal fatto che i tedeschi vengono descritti come dei «macellai» (346) e dal fatto che si dica che Vlasov avrebbe dovuto ritenersi orgoglioso del proprio razionalismo

che era davvero una specie di coraggio [...] capace di reggere il paragone con il nobile ateismo bolscevico. (336)

Ancora, all'inizio di «Sortita» si legge che

durante la ritirata della XXXII Armata verso Mosca, certi dispacci segreti [...] avevano dato modo a Vlasov di tornare in un villaggio da loro stessi evacuato un'ora prima. Mi dispiace dover dire che trovò i contadini, in totale spregio del potere sovietico, già intenti a preparare il pane e il sale del tradizionale benvenuto, che intendevano evidentemente offrire ai fascisti in arrivo. (342)

Quando però Vlasov diserta passando dalla parte dei nazisti, il narratore cambia e si riferisce al «nostro Führer» (358), alla «nostra Grande Germania» (364), e le donne sovietiche vengono descritte come «creature appartenenti al tipo razziale slavo» (366).

In seguito, dopo che nel gennaio del 1945

i russi attraversarono i confini del Vecchio Reich e misero piede sul nostro suolo patrio (407)

e Vlasov viene arrestato, processato e giustiziato dall'esercito sovietico, il narratore cambia nuovamente, ritornando ad utilizzare un punto di vista sovietico: 
Potremmo dire che il suo errore fu il cosmopolitismo, definito dalla Grande enciclopedia sovietica come l'ideologia reazionario-borghese del cosiddetto 'cittadino del mondo'. Il cosmopolitismo pretende di conciliare tutto. In realtà non è che un paravento capace di nascondere le aggressive pulsioni transnazionali del capitale [...]. Il 2 agosto 1946 le 'Izvestija' annunciarono che, in base all'articolo 11 del nostro codice penale, la condanna a morte del traditore A. A. Vlasov era stata eseguita. (416-17)

In Europe Central quindi da un lato l'assegnazione di una singola storia a ciascun frammento narrativo impedisce «l'emergere di un centro privilegiato del discorso» (Ercolino 2014, 99), e dall'altro questo

costituisce una piattaforma morfologica ideale per l'innesto e lo sviluppo di una narrazione corale. (99)

Centrali nel romanzo di Vollmann sono infatti, come già ribadito, «una collettività di personaggi e una pluralità di storie» (99), che come vedremo sono mantenute insieme tra loro da un profondo significato morale e allegorico.

Come è stato sottolineato la gestione di questo tipo di narrazione massimalista sembrerebbe allora affidata in Europe Central a un particolare tipo di narratore, la cui voce

è la risultante della somma delle informazioni narrative veicolate da ciascuna delle focalizzazioni presenti nelle diverse unità in cui si articola la diegesi. (Ercolino 2014, 167)

Come notato in un recente studio, l'alternarsi delle voci narranti all'interno del romanzo sembra del resto suggerire l'assenza di un vero e proprio narratore principale, che potrebbe coincidere con l'autore (Malvestio 2019, 106-7): per Vollmann infatti «lo scrittore [è] reso divino dalla propria assenza» $(2010,28)$, e le sue occasionali apparizioni all'interno del romanzo non possono essere interpretate come strategie metanarrative (Malvestio 2019, 107), perché contribuiscono piuttosto al rafforzamento di quel portato etico-morale che Vollmann vuole trasmettere attraverso le parabole dei suoi personaggi:

In Europe Central [...] Vollmann inserts the parenthetical statement '(I'm writing in the year 2002)' into a parabolic sketch of Hitler's ascension to Reich Chancellor, explicitly puncturing the illusion of his own fiction. As Michael Hemmingson points out in his critical study on Vollmann, 'Vollmann does not appear in [Europe Central] as meta-character, alter-ego, or himself - although one may wonder who the intermittent ' $\mathrm{I}$ ' is who pops up now and then'. Vollmann's first-person narrators - one of them a ruthless Nazi, the other a 
Soviet ideologue - further distance Vollmann from the perceived 'moral' of his parables, creating a story-within-a-story framework that adds yet another level of self-awareness. (Santin 2015, 146)

Si tratta di una forma di onniscienza ottenuta tramite la

ricomposizione dei singoli punti di vista adottati di volta in volta dal narratore a livello micro-strutturale, in una focalizzazione zero derivata che si definisce, invece, sul piano macro-strutturale. (Ercolino 2014, 167)

È quindi considerando i due diversi piani, quello del singolo frammento, «il livello, cioè, micro-strutturale» (165) e quello della narrazione nel suo complesso, che rappresenta «il livello macro-strutturale» (165), che si può cogliere

[l']elementare bisogno [...] di costruire uno sguardo narratoriale in grado di percepire dall'alto, di dominare, l'intero flusso narrativo. (168)

È infatti possibile considerare l'onniscienza come quella modalità della narrazione che si adatta «più efficacemente di altre al controllo del materiale narrativo» (168): questa permetterebbe infatti all'autore di non intervenire nelle vicende narrate, garantendo un confronto 'senza intermediari' tra la vicenda e il lettore. Adottare questo tipo di prospettiva narrativa presuppone una sorta di «moral nonintervention» (Coffman 2015, 15) da parte di Vollmann, il quale si rifiuta, da parte sua, di fornire anche il minimo tipo di giudizio rispetto alle vicende narrate (Malvestio 2019, 107).

\subsection{Compiutezza}

Ercolino individua all'interno dei romanzi massimalisti delle strutture capaci di controllarne l'esuberanza narrative e di garantirne la 'compiutezza' formale. In particolare sono le «strutture concettuali» (Ercolino 2014, 143) del 'mito' e del 'leitmotiv' a risultare fruttuose se applicate alla narrazione di Europe Central.

Come scriveva Eliot a proposito dell'Ulisse di Joyce, l'uso del mito è

un modo di controllare, di ordinare, di dare una forma e un significato all'immenso panorama di futilità e anarchia che è la storia contemporanea. (Ercolino 2014, 150)

Anche il romanzo massimalista ricorre al metodo mitico «per conferire un ordine al materiale narrativo» (150): in Europe Central Voll- 
mann ne fa un uso massiccio a supporto dell'intera narrazione, utilizzandolo anche in maniera complementare per illuminare con grande efficacia il retroterra ideologico nazista. Questo accade in particolare quando l'autore decide di rappresentare direttamente la figura di Hitler: in questo caso il paradigma mitico a supporto della Weltanschauung nazista viene evocato nel capitolo centrale, «Il sonnambulo» - il soprannome che Vollmann riserva a Hitler -, quando la narrazione della saga dei Nibelunghi nella sua interpretazione wagneriana si sovrappone alla parabola di morte della Germania nazista.

$\grave{E}$ in questo modo che Vollmann descrive il legame a doppio filo tra la storia mitica dei fratelli Gunnar e Hogni e le prime campagne militari naziste:

Guthrún, ridotta in schiavitù e incatenata agli Unni dal matrimonio, al di là della foresta oscura, mandò ai fratelli Gunnar e Hogni un anello al quale aveva avvolto un pelo di lupo per avvertirli di stare alla larga, ma certi stratagemmi non hanno un'efficacia garantita neppure nei sogni. [Ma] i due fratelli accettarono l'invito unno. Non potevano fare altrimenti, essendo intrappolati, come dicevo, in un sogno fatale [...]. Quando nel giorno decisivo del 1936 il cancelliere tedesco, un certo Adolf Hitler, ordina a venticinquemila soldati di attraversare sei ponti e di occupare la zona della Renania, anche lui teme il futuro. A differenza di Gunnar, è pallido in volto [...]. Che cosa risponderanno gli inglesi? Nulla [...]. E arriva l'ultimatum! [...] Al che, per i suoi vassalli e tirapiedi, a Monaco, intona: 'Seguo la via dettata dalla Provvidenza, con la sicurezza di un sonnambulo'. I presenti lo applaudono. Le vergini unne dalle braccia candide strillano di gioia. $(2010,164-5)$

La Germania invade la Polonia, e Hitler

Esita di nuovo. Ha paura di ciò che gli sta di fronte nella foresta di Myrkvith [...]. Immagina come dev'essersi sentito Gunnar quando gli Unni lo gettarono nella fossa dei serpenti. (166-7)

La visione allucinata continua rapida e frenetica, si sposta sulla rappresentazione wagneriana dell'epopea dei Nibelunghi: quando attacca la Russia nel 1941, Hitler si chiede

Quanto ci vorrà per ridurre quell'impero a una macchia sotto lo stivale? Tre settimane, probabilmente, ma a questo mondo non sempre l'esattezza è possibile. A Bayeruth, ad esempio, Das Rheingold è stato rappresentato in due ore e tre quarti, ma in alcuni casi dura fino a tre ore [...]. [Ma] la Russia gli si avventerà contro come il serpente-dragone che si solleverà alla fine del mondo, recando cadaveri tra i suoi artigli. (171) 
Fino ad arrivare all'«ora della grande battaglia tra Sigmund e Hunding» (176), il drammatico epilogo che attende Berlino, costretta da Hitler a difendersi fino all'ultimo sangue alle forze sovietiche che hanno ormai preso la capitale del Reich:

Ora comprende, con tutta l'anima, la ragione per cui Gunnar e Hogni non avevano potuto resistere all'invito degli Unni: nonostante lasciassero presagire rovina, e sventura per la loro sorella, almeno avrebbero conquistato quel brillante, benché sinistro, momento di luce quando si sarebbero avvicinati alle piazze d'armi dei loro nemici [...]. Il sonnambulo borbotta, come aveva fatto alla vigilia della campagna di Russia: 'Il mondo resterà con il fiato sospeso'. (176-7)

La Germania nazista è agonizzante, e Vollmann descrive per l'ultima volta il delirio di Hitler:

Quando calarono Gunnar nella viscida segreta delle vipere, lui suonò la sua arpa con tale grazia da far addormentare tutti i serpenti. Alla fine, però si stancò, e dal groviglio di rettili su cui fu costretto a distendersi uno se ne levò a mordergli il fegato; e così perì in quella tenebra di serpenti. Ben conoscendo il proprio dovere, Guthrún servì il cuore dei propri figli al marito che le aveva trucidato i fratelli. Dopo di che incendiò il castello, radendolo al suolo. Il sonnambulo [...] anela a essere un altro Gunnar. Non è forse un arpista anche lui? Non è, forse, stato abituato a incantare tutti i serpenti fino a quel momento? E la sua Germania, sarà Guthrún. La Germania deve morire feroce, mettendo a fuoco ogni cosa... (178-9)

A margine dell'analisi sul mito come elemento di compiutezza del romanzo massimalista, è interessante rilevare che lo status di 'sonnambulo' che Vollmann attribuisce a Hitler connota una dimensione onirica collocabile oltre il significato della narrazione in senso lato. Come la proliferazione di altre immagini e metafore mitologiche che compaiono in Europe Central, lo 'stato di sonno' riflette infatti l'irrazionale sotteso al fascismo, e la sua ambizione di voler creare una dimensione astorica e atemporale (Malvestio 2019, 117). È stato infatti osservato che "the elimination of historical time in favor of a mythical, circular time is a key feature of fascist ideology that Vollmann demonstrates to have clearly understood» (117), e tale aspetto è evidente nel modo in cui l'autore mette in scena le allucinazioni di Hitler. Secondo l'autorappresentazione nazista che ne risulta ad emergere è un vero e proprio percorso iniziatico, che fondendosi con il mito vuole condurre alla conoscenza di una verità assoluta (117); ; ed è, in questo caso, la visione che spinge la Germania alla guerra. 
Si tratta di quello che Károly Kerényi ha definito 'mito tecnicizzato', ovvero quel mito non genuino evocato 'intenzionalmente' dall'uomo allo scopo di ottenere determinati poteri politici: secondo Furio Jesi, a cui si devono i principali studi sulla cosiddetta 'macchina mitologica', le immagini così evocate vengono

deformate nel senso delle finalità dei tecnicizzatori per il fatto stesso di essere state evocate intenzionalmente da essi, e non prescelte spontaneamente dal flusso mitico. Esse, dunque, costituiscono una realtà linguistica particolarmente soggettiva, come soggettivo è il cosmo di chi - nelle parole di Eraclito - è in 'stato di sonno'. (Jesi 1968, 36)

La condizione sonnambolica attribuita da Vollmann a Hitler sembra pertanto una caratteristica adeguata per descrivere lo 'status' di chi usa il mito tecnicizzato, che a differenza del mito genuino ${ }^{3}$ evoca delle immagini mitiche e le deforma 'soggettivamente': immagini che contrastano e indeboliscono la coscienza e mettono in pericolo tanto il linguaggio quanto l'equilibrio tra coscienza e inconscio. La realtà linguistica nata dal mito tecnicizzato non può infatti possedere un valore collettivo perché il suo flusso mitico ha subito una manipolazione ad opera del tecnicizzatore, e quindi il linguaggio del mito non è più quello di una collettività ma appartiene invece a un ristretto gruppo sociale. Il mito tecnicizzato,

sopprimendo il valore collettivo e oggettivo del processo conoscitivo e linguistico, riconduce l'uomo allo «stato di sonno» e apre la via al predominio dell'inconscio. Se, infatti, l'inconscio è di per sé collettivo - secondo l'insegnamento di C. G. Jung -, esso si mantiene in equilibrio nella psiche umana quando entra in rapporto con un altro elemento collettivo quale la coscienza in 'stato di veglia'; ma è destinato a prevelare se nella psiche la coscienza è in 'stato di sonno', e cioè viziata dal soggettivismo. (Jesi 1968, 37)

In Europe Central l'evocazione del mito dei Nibelunghi ad opera del sonnambulo è caratterizzata da un rapporto distorto con un passato «tanto remoto da poter essere identificato con un eterno presente» (Jesi 1968, 37): questo rapporto è viziato perché determinato da un'evocazione manipolata del mito, e per questo il passato che sopravvive nelle immagini che si affacciano alla mente di Hitler è una «sopravvivenza deforme, soggettiva» (37), in cui il tecnicizzatore in stato di

3 Secondo Jesi il mito genuino sgorga «spontaneamente dalle profondità della psiche» $(1968,35)$, opponendosi come una barriera contro il "predominio indiscriminato dell'inconscio» (36). Una facoltà che coincide con il carattere 'oggettivo' delle immagini evocate dal mito genuino stesso. 
sonno proietta le proprie paranoie per poter disporre in questo modo di «un 'precedente' mitico di ess[e]» (37), un precedente utile come strumento politico. In balia del flusso mitico ormai fuori controllo, consapevole della disfatta imminente, al sonnambulo si manifesta allora la personificazione mitica delle proprie colpe:

Una donna altissima si staglia davanti a lui. Le arriva a malapena alle ginocchia. Le pupille di lei paiono scintille originate dalle punte di lancia delle Valchirie [...]. Lui allora capisce di essere sotto processo [...]. Sognando una risposta a ciò che lei non ha ancora detto, le spiega che nelle opere lo sforzo più nobile compiuto da Wotan è quello di soppiantare se stesso. Non gli importa di perdere la guerra, purché gli riesca di impedire agli ebrei di riprendersi l'anello magico [...]. Chi ti ha generata? 'Fuoco' è mio padre. 'Catastrofe' è il nome di mia madre. E per quale ragione sei qui ad attendermi? Per dirti quel che sai da sempre: che sei nato colpevole e soggetto a forze superiori; che il nulla per cui tu ardi si rifiuta di accoglierti; che gli antichi tesori si corrompono al tuo tocco [...]. [La donna scoppia in] una risata, simile a un crepitio di futuro, come ossa che sbatacchiano entro una processione di bare sulla terra carbonizzata della Auschwitz liberata [...]. Lui, allora, in tono implorante, sussurra: Perché mi avete messo al mondo? Vorrei non essere mai nato... (Vollmann 2010, 174-5)

Secondo Jesi proprio questo è stato il passato mitico per il nazismo, il cui linguaggio ha coinciso con

l'inabissarsi nelle immagini deformi del passato offerte dalla tecnicizzazione del mito. $(1968,40)$

In altri termini, quel tipo di linguaggio valido solo per una cerchia ristretta di razza pura attingeva a immagini mitiche deformate, in cui

ciascuno si abbandonava ciecamente al flusso dell'inconscio, ritrovando in quell'esoterismo mostruoso una conferma ed un precedente mitico delle proprie malattie e delle proprie colpe. (40)

\section{Così}

Se il denominatore comune di un gruppo sociale è la colpa e la volontà colpevole, e se [...] quest'opera 'dall'alto' si esplica anche nel fornire miti deformati ad hoc e quindi nel favorire il predominio delle 'forze oscure' sulla coscienza, si può ben parlare di 'stato di sonno' per chi fa parte di tale gruppo. Ognuno dei dormienti parla il proprio linguaggio: proprio, in quanto privo di obiettività e deformato secondo le proprie colpe. (40) 
La seconda struttura concettuale che regola l'esuberanza narrativa di Europe Central è quella del leitmotiv. In particolare il leitmotiv che più contribuisce a conferire ordine e significato al materiale narrativo del romanzo è quello della scelta che deve compiere il personaggio in un momento cruciale della sua vita, il quale può trovarsi in bilico tra la fiducia nel proprio genio e il suo ripudio per soddisfare il totalitarismo che lo annichilisce - Šostakovič e le accuse di formalismo e di «oscurantismo borghese» (Vollmann 2010, 211) - oppure incerto tra l'obbedienza ad obblighi militari sempre più deliranti o la vigliaccheria di una resa al nemico - il feldmaresciallo Paulus e la sacca della VI Armata, il generale Vlasov e l'assedio di Leningrado. È stato già evidenziato che l'obiettivo dichiarato espressamente da Vollmann è quello di scrivere delle parabole su «attori morali» famosi, famigerati o anonimi «osservati nei momenti di importanti decisioni» (963): è a questo leitmotiv - che rende ambigui e tragici i personaggi che lo vivono nel corso della narrazione - che si collega il tentativo di Vollmann di 'defamiliarizzare' la vicenda storica, per offrire al lettore un punto di osservazione sugli eventi da una prospettiva obliqua. Il lettore viene così messo in una scomoda posizione e invitato a riflettere, in sostanza, sulle scelte che avrebbe potuto compiere egli stesso se si fosse trovato al posto del personaggio: «E tu, lettore, che cosa avresti scelto?» (278). E a questo che si riferisce Ercolino quando, a proposito del 'realismo ibrido' del romanzo massimalista, sostiene che il compito dello scrittore è quello di

recuperare alla mimesi zone della realtà inesplorate dal realismo classico, nella speranza di produrre un effetto straniante sul lettore. Il nuovo realismo, un realismo critico, deve puntare paradossalmente a una sorta di defamiliarizzazione dell'esistente, sulla scorta di uno slancio etico poderoso. $(2014,260)$

Quello della scelta morale è il leitmotiv che compare dunque nel corso di tutto il romanzo di Vollmann, ma è in «Mani pulite» che rivela il suo valore morale più alto. La vicenda dell'Obersturmführer Kurt Gerstein, il quale tentò clandestinamente di opporsi allo sterminio nazista nei campi di concentramento di Belzec e Treblinka, diventa la parabola emblematica della responsabilità dell'uomo al cospetto della tragedia della Shoah e dei dilemmi morali che è chiamato ad affrontare per salvaguardare la propria umanità. Gerstein sembra pertanto rappresentare per Vollmann un eccezionale esempio di perseveranza morale in nome della giustizia:

È naturale credere, o voler credere, dato che l'inerzia è autoconservazione, che dopo aver scoperchiato la botola [sulle camere a gas] non sarà necessario assumersi il rischio di riportare altro al- 
la luce. Il fascino della comodità, che impedì a gran parte dei tedeschi di fare quel che fece Gerstein, ci spinge a dire: Io, comunque, ho fatto la mia parte. Ora tocca agli altri. (Vollmann 2010, 546)

\section{Tuttavia il dilemma morale di Gerstein}

is not characterized by an obvious choice between 'right' and 'wrong', which is a recipe for melodrama, but by an exigently tragic collision between 'right' and 'right'; he must make a crushing moral choice between saving the lives of innocent Jews while also keeping his family safe. This properly tragic dimension in Vollmann's parable disrupts traditional, Kantian-descended moral philosophy's attempt to posit a systematically coherent ethical theory. (Santin 2015, 141-2)

Prestando servizio a Belzec e assistendo agli orrori che si compivano nei Lager, l'ufficiale nazista tenta in ogni modo di contattare esponenti religiosi e ambasciatori di stati neutrali per fornire la sua testimonianza, mettendo a rischio la propria incolumità ed esponendosi volontariamente alle mostruosità degli eccedi: quando la moglie chiederà all'SS

'Come puoi, tu che sei un uomo di sani principi e un sincero cristiano, assistere senza battere ciglio a certe cose?',

la risposta di Gerstein, «Con il volto indurito come carne da elmetto», sarà:

'Sono felice di aver visto queste atrocità con i miei occhi, perché un giorno, a Dio piacendo, potrò darne testimonianza'. (Vollmann 2010, 562)

\section{L'SS infatti}

si costrinse a essere testimone di tutto: in modo maniacale, contò i fili spinati della recinzione di un ghetto di transito... un'altra nota a piè di pagina per la sua dichiarazione gratuita ancora da scrivere. Vide l'ammasso scuro di ebrei polacchi, seduti nella piazza del mercato della loro città; aspettavano le $S S$ e la polizia che li avrebbero circondati e scortati verso la morte. Mentre i suoi colleghi li sorvegliavano, Gerstein li contò: altri 724 crimini da riportare nel suo affidavit. E poi, dato che il suo permesso timbrato dall'ufficio di Eichmann gli consentiva di muoversi quasi a suo piacimento, accompagnò i camion, per vedere dove si trovavano le fosse comuni. Era presente e si sforzò di ridere con i suoi camerati quando denudarono e picchiarono gli ebrei, prima di sparare loro. (578-9) 
Gerstein è combattuto tra la malvagità delle violenze - a cui assiste e a cui deve contribuire, con l'approvvigionamento dell'acido prussico e dello Zyklon B per le camere a gas - e la propria coscienza che lo spinge a non perdere la ragione e a resistere per testimoniare, un giorno:

Fatti coraggio, Kurt. È la tua coscienza che ti parla. Quando ti troverai lungo la via oscura, ricordati di me, e agisci sempre per il meglio. (537)

Il suo conflitto interiore allora diventa la lotta di un individuo contro una volontà superiore, la volontà del Reich, metafora del divino, come gli ricorda il padre:

Il punto è che noi non dovremmo avere l'impertinenza di calarci nella parte di Dio. Non spetta a noi decidere chi deve vivere e chi morire [...]. Ho fatto il mio dovere, e non mi sono mai messo nei panni di Dio. Ho sempre avuto quell'umiltà che si conviene a un essere umano.

E non credete che dovremmo poter scegliere ognuno per sé?

Come hanno scelto Adamo ed Eva, contro il comandamento divino? [...]

Kurt Gerstein sussurrò: Dobbiamo poter scegliere come ha scelto Gesù.

No, ribatté il padre, questa è fantasia bella e buona. Tu non sei Gesù. Quel che immagini è impossibile. (585-6)

Oltre a rappresentare un motivo ricorrente allora, quello della 'scelta' è un leitmotiv dal profondo significato morale, la cui portata travalica i confini del romanzo facendosi «veicolo di un contenuto dalla marcata valenza utopica» (Ercolino 2014, 147): per Vollmann Kurt Gerstein rappresenta un eroe tragico non tanto per il risultato delle sue azioni, ma perché rifiuta di fare suo il concetto di «impossibilità per far[si] scudo contro qualsiasi tipo di impegno [morale]» (2010, 599). Esempio emblematico della radicale empatia dell'autore, «Mani pulite» è una parabola che se compresa nella sua portata tragica rappresenta la «dynamic relationship between Vollmann's fiction and his overall ethical vision» (Santin 2015, 160).

\subsection{Impegno etico}

Nel 1992 Vollmann pubblica sul numero 15 della rivista Conjunctions un vademecum intitolato «American Writing Today: A Diagnosis of the Disease», in cui formula delle critiche rivolte alla società americana, che reputa incapace di provare empatia e di comprendere le esigenze di un 'altro' identificato con le minoranze etniche, gli 
emarginati, gli ultimi nella scala sociale: per Vollmann «our failures illuminate us as Selves incapable of comprehending others» (1992). Dello stesso tono sono le accuse che rivolge alla letteratura postmoderna di quegli anni, che l'autore prende di mira:

In this period of our literature we are producing mainly insular works [...]. Here the Self is projected and replicated into a monotonous army which marches through story after story like deadly locusts. Consider, too, the structuralist smog that has hovered so long over our universities, permitting only games of stifling breathlessness. (The so-called New Historicism promises no better). (Vollmann 1992)

Vollmann appartiene a una generazione di romanzieri ritrovatisi in una situazione complessa e contraddittoria rispetto ai padri della narrativa postmoderna (Malvestio 2019, 132). Lui e gli autori della sua generazione - David Foster Wallace e Jonathan Franzen tra gli altri - ne hanno infatti sicuramente riconosciuto l'eredità, rielaborandone la sperimentazione stilistica, ma al tempo stesso ne hanno anche contestato l'eccessiva autoriflessività e il cinismo, rispetto ai quali hanno contrapposto invece una prosa che ambisse in qualche modo alla sincerità, all'empatia nei confronti del lettore, per affrontare costruttivamente i problemi della contemporaneità. In un articolo pubblicato nel 1997, E Unibus Pluram: gli scrittori americani e la televisione, riflettendo sullo statuto complesso e frammentario di una società - e di una scrittura - potentemente influenzata dallo schermo televisivo, Foster Wallace si interrogava sulle prospettive del romanzo che avrebbe dovuto succedersi alla letteratura postmoderna. Secondo Wallace:

I veri futuri «ribelli» letterari in questo paese [potrebbero occuparsi] dei problemi e delle emozioni poco trendy della vita quotidiana americana con rispetto e convinzione. [...] I nuovi ribelli potrebbero essere artisti pronti a rischiare lo sbadiglio, gli occhi al cielo, il sorriso di sufficienza, le strizzatine d'occhio, la parodia dei fini umoristi, i «Dio mio quant'è banale». A rischiare di essere accusati di sentimentalismo, di melodrammaticità. Di eccessiva sprovvedutezza. Di debolezza. $(1999,54)$

In maniera non dissimile anche il vademecum di Vollmann accusa la scrittura postmoderna di divertissement disimpegnato e nichilista, a cui contrappone un'idea di letteratura improntata all'apertura nei confronti dell'Altro:

Art takes us inside other minds [...]. Of all the arts, although photography presents best, painting and music convey best, and sculpture looms best, I believe that literature articulates best. (Vollmann 1992) 
Vollmann propone una lista di regole in sette punti, ideali riferimenti sui quali costruire una nuova scrittura:

1. We should never write without feeling.

2. Unless we are much more interesting than we imagine we are, we should strive to feel not only about Self, but also about Other. Not the vacuum so often between Self and Other. Not the unworthiness of Other. Not the Other as a negation or eclipse of Self. Not even about the Other exclusive of Self, because that is but a trickster-egoist's way of worshiping Self secretly. We must treat Self and Other as equal partners. (Of course I am suggesting nothing new. I do not mean to suggest anything new. Health is more important than novelty.)

3. We should portray important human problems.

4. We should seek for solutions to those problems. Whether or not we find them, the seeking will deepen the portrait.

5. We should know our subject, treating it with the respect with which Self must treat Other. We should know it in all senses, until our eyes are bleary from seeing it, our ears ring from listening to it, our muscles ache from embracing it, our gonads are raw from making love to it. (If this sounds pompous, it is perhaps because I wear thick spectacles.)

6. We should believe that truth exists.

7. We should aim to benefit others in addition to ourselves. (Vollmann 1992)

A una scrittura formalmente complessa ed enciclopedica, di derivazione postmoderna, Vollmann accosta quindi una profonda attenzione orientata alla dimensione etica; si tratta di una presa di posizione che come noto ha caratterizzato anche la vita personale di Vollmann, il quale nel corso della sua carriera si è dedicato all'esplorazione del mondo degli emarginati, della prostituzione e della tossicodipendenza - da cui è nata la cosiddetta 'Prostitution Trilogy': Puttane per gloria (2000), Storie di farfalle (1999), The Royal Family (2000). Ancora, nel 1982 Vollmann ha deciso di partire per l'Afghanistan e combattere al fianco dei Mujaheddin - Afghanistan Picture Show, ovvero come ho salvato il mondo (2005) -, fino a decidere in tempi più recenti di sperimentare il cross-dressing - esperienza narrata in The Book of Dolores (2013).

Non a caso quindi la narrazione di Europe Central rappresenta quella che Kiš ha definito una «scelta totale», un «atto umanistico» $(2009,128)$ il cui campo tematico è

monopolizzato da temi di grande rilevanza storica, politica e sociale: temi massimi e ricorrenti, (Ercolino 2014, 219) 
aspetti fondamentali dell'impegno etico' che secondo Ercolino caratterizza il romanzo massimalista. Un impegno etico tutt'altro che memorialistico o celebrativo: se ad esempio quella di Binet è un'operazione di tipo commemorativo e a tratti enfatica, in cui i protagonisti dell'Operazione Antropoide vengono definiti come

gli autori di uno dei più grandi atti di resistenza della storia umana, e indiscutibilmente della più eroica impresa di resistenza della Seconda guerra mondiale, (Binet 2011, 5)

quella di Europe Central è invece una scrittura antiretorica, costruita con l'intento di mettere in luce l'ambiguità morale di uomini e donne a cui viene affidata «una gigantesca responsabilità morale» (Vollmann 2010,44) nel momento in cui sono chiamati dalla Storia a prendere delle decisioni cruciali. Di conseguenza, questo significa anche che i personaggi delle parabole di Europe Central non sono tanto la rappresentazione di quegli alti valori che per esempio Binet in $\mathrm{HHhH}$ attribuiva ai suoi protagonisti - come possono essere la figura della partigiana Zoja o del compositore Šostakovič in Europe Central - ma sono soprattutto figure enigmatiche e controverse come quella dell'ufficiale delle SS Gerstein, del generale tedesco Paulus o del sovietico Vlasov. Estremizzando questa prospettiva, personaggi e vicende in Europe Central si fanno allora universali: il contesto traumatico delle guerre e degli stermini del Novecento diventa una cornice simbolica e i personaggi che la abitano rappresentano la personificazione dei quesiti etici che Vollmann rivolge al lettore, interessato all'osservazione dell'uomo nei momenti cruciali in cui le esistenze dei singoli si intrecciano ai destini generali.

Lo scopo di Vollmann è perciò quello di mostrare i personaggi nel momento critico di una decisione, piuttosto che il contesto in cui questa avviene (Malvestio 2019, 103); sono i processi psicologici che accompagnano la scelta a interessare la sua narrazione, più che le cause - e le conseguenze - che l'hanno creata (103). Da qui il fatto che le vicende di Europe Central implicano nel lettore una 'ricezione emotiva' della vicenda narrata, piuttosto che un rapporto intellettuale con essa, perché come abbiamo potuto osservare a proposito di «American Writing Today: A Diagnosis of the Disease» secondo l'etica di Vollmann è proprio la sfera dell'empatia a permettere una profonda comprensione delle vicende storiche e del loro impatto sull'individuo.

Con le sue potenzialità la grande letteratura secondo Vollmann deve essere interessata alla verità, il che si traduce nella comprensione delle circostanze in cui agiscono i personaggi, i cui codici di comportamento fino a prova contraria «must be presumed to be good enough for them» (Vollmann 2003, 3: 122). A questo proposito Christopher Coffman ha scritto che 
Rather than permitting us to recognize that even those who seem most terrifyingly foreign or most boringly familiar are basically people very much like us, [Vollmann] asks that we accept them on their terms, which may need to be recognized as finally incomprehensible and even dangerous. One might quickly observe that it is this radical openness that allows Vollmann's empathetic presentations of such disturbing figures as [...] several of the Nazis of Europe Central. At its limit, this encounter with the essential foreignness of others may be disorienting and disheartening. We are, as Vollmann puts it, 'Lonely Atoms', always at least partially unknown and unknowing, and the impact of that realization is exacerbated by the fact it comes to us at the very moment in which the self is most vulnerable - openness admits discomfort and suffering as concomitants of knowledge and pleasure. $(2015,14)$

Nei sette volumi di Rising Up and Rising Down (2003), la monumentale analisi sull'uso e il significato della violenza nella società, Vollmann attesta chiaramente questo tipo di approccio, in una sorta di dichiarazione di intenti:

No credo will eliminate murder. But if we think about a sufficient number of cases we may be able to plant the seeds of a tentative ethics which others could consider, pick and choose from and hopefully benefit from even if they cannot improve. That is my hope for this book. I know that other people's advice has rarely made me better than I was. When it has, it was less often the advice itself than the spirit in which it was given which helped me, requiring me out of sheer respectful reciprocity to listen, search and consider. (2003, 1: 38)

Anche per i personaggi più disturbanti che compaiono in Europe Central dunque il principio guida (Malvestio 2019, 108) resta quello del «no judgment» (Vollmann 2003, 1: 44):

My policy will always be to treat with empathy and respect anyone agreeing to be studied, interviewed, exposed. I would have been courteous to Eichmann. (2003, 1: 50)

Si tratta evidentemente di una prospettiva quanto meno scomoda, rischiosa, che però se applicata a Europe Central può contribuire a fornire una spiegazione tanto dell'eclissarsi dell'autore rispetto alla narrazione quanto della completa aderenza dei diversi narratori intradiegetici all'ambiente politico che li circonda. Ecco allora il significato delle 'parabole' a cui si riferisce Vollmann, che mirano a una comprensione delle circostanze morali, etiche presenti nello spirito del tempo e gettano una diversa luce sul significato della Seconda 
guerra mondiale, «not through an intellectual statement, but through an empathic reconstruction» (Malvestio 2019, 108), che costringe il lettore a porsi allo stesso livello dei tragici personaggi del romanzo.

Le parabole che compongono Europe Central, tenute insieme dal loro significato morale, custodiscono allora un messaggio rivolto al lettore, l'invito a una riflessione di tipo metastorico (Malvestio 2019, 105): considerare come le storie che caratterizzano il romanzo possano relazionarsi alla sua esperienza, per tentare successivamente la strada del cambiamento personale. In questi termini il capitolo «I salvatori: un racconto cabalistico» può essere letto come un passaggio programmatico sulla potenza trasformatrice della narrazione, che sola può veicolare la possibilità del cambiamento attraverso lo sguardo obliquo dell'empatia:

I critici letterari, perlopiù, concordano nell'affermare che la prosa narrativa non si possa ridurre a mera falsità. I protagonisti ben delineati prendono vita, la pornografia causa orgasmi e fingere che l'esistenza sia come la vogliamo potrebbe produrre, con ragionevolezza, la condizione desiderata. Di qui, le parabole religiose, il realismo socialista, la propaganda nazista. E se, in modo analogo, anche questo racconto brulica di sovrannaturalismo reazionario, si potrebbe imputare al desiderio dell'autore di vedere lettere che sgattaiolano sui soffitti, cominciando con cautela a reificarsi in forma di angeli. E se le lettere sono in grado di farlo, perché non dovremmo riuscirci noi? (Vollmann 2010, 45) ry Fiction di Marco Malvestio (2019) (2021-07-05). 



\section{Romance e Shoah}

Sommario 4.1 Romance e Shoah. - 4.2 Enric Marco e l'industria della memoria. 4.3 Nostalgia del trauma e Reality Hunger.

\subsection{Romance e Shoah}

Romance e Shoah sembrano due termini incompatibili. Si potrebbe pensare che sia addirittura offensivo per le vittime di questa enorme tragedia storica ragionare sui temi del romance, che spesso è associato alla letteratura di intrattenimento, persino di basso profilo. Tuttavia, pur non dimenticando mai la realtà storica di quanto è accaduto, è necessario, tanto più nel tempo della postmemoria, ragionare sulle motivazioni tecniche sulle quali si fondano i racconti della Shoah, che, com'è noto, pare godano oggi di maggior fortuna rispetto a tempi passati. Solo partendo dalle basi costitutive di questo rapporto è possibile comprendere ciò che nel capitolo «Postmoderno e Shoah» abbiamo chiamato esorcismi rispetto alle pratiche di storytelling, e che nel capitolo successivo indagheremo analizzando la prospettiva della narrazione memoriale compilata dai testimoni diretti.

Come si sa, la teoria letteraria distingue da tempo tra novel e romance, celebre è per esempio la definizione di Walter Scott che nel suo Essay on Romance del 1824, scrive:

Saremmo piuttosto propensi a descrivere il romance come una narrazione fittizia in prosa o in versi, il cui interesse si impernia su fatti inconsueti e meravigliosi, contrapponendolo così al termine affine 'romanzo' (novel) che Johnson ha descritto come 'un racconto scorrevole generalmente d'amore', ma che noi piuttosto defi- 
niremmo come una narrazione fittizia che differisce dal romance perché i fatti si adattano al corso ordinario delle vicende umane e al moderno assetto della società (Scott [1834] 1983, 200)

Il novel nasce come romanzo sentimentale, ma già all'inizio dell'Ottocento vira il suo significato nella direzione indicata da Scott, ovvero: nel novel «i fatti si adattano al corso ordinario delle vicende umane e al moderno assetto della società», il romance invece «si impernia su fatti inconsueti e meravigliosi». Per dirla in modo ancora più tranchant, il novel parla della realtà, il romance di eventi chiaramente fittizi. Questa distinzione tra eventi reali e finzionali era già stata proposta in un'epoca ancora precedente e in relazione al romanzo storico da Charles Brockden Brown, nel suo celebre saggio «The Difference Between History and Romance», dove il secondo termine è definito come «a tissue of untruths [...] which never had existence» (Brown 2009, 341).

È ovvio allora che nel caso della scrittura memorialistica siamo nei territori del novel, se non oltre questi territori: vicini a quella che Brockden Brown chiamava history e che forse si dovrebbe definire meglio come storiografia. In generale è difficile che il romanzo storico, quando rigorosamente fondato su fatti reali, sconfini nel romance al quale pertengono più il romanzo fantastico, il fantasy, l'horror o altri generi chiaramente fittizi. Solitamente il romanzo storico che diventi palesemente romance è considerato un prodotto di largo consumo e di serie B, proprio per l'approssimazione con la quale utilizza le fonti o le piega al sensazionalismo. Anche se è ancora Scott a riconoscere la presenza di un confine labile, non sempre chiaramente definibile tra storiografia e romance: «[romances and real history] may be termed either romantic histories, or historical romances, according to the proportion in which their truth is debased by fiction, or their fiction mingled with truth» (Scott 1834, 134). Una proporzione forse mai definibile con certezza, ma che certamente non può ignorare totalmente le regole del romance.

Cercando di individuare un discrimine di tipo cronologico, possiamo dire che nel panorama letterario contemporaneo il romance si sia evoluto in forme e declinazioni nuove dalla fine degli anni Cinquanta del Novecento in poi, cioè da quando il postmoderno ha iniziato ad indagare e descrivere la dimensione del reale rispondendo alle esigenze opposte con cui, prima, si articolava la scrittura del Naturalismo francese, o il grande romanzo ottocentesco. Allo stesso tempo, si è assistito ad un altro fenomeno che riguarda più da vicino la concezione e la trasmissione degli eventi storici da parte dell'individuo. Il concetto di 'storia' in sé è andato a infatti a modificarsi, avvicinandosi di più a quel tipo di sviluppo narrativo degli eventi proprio della letteratura tout court: poco prima dell'inizio del nuovo millennio, la disciplina storica è stata ripensata e osservata più come 'conoscen- 
za testuale' che resoconto di contenuti di tipo scientifico da parte della comunità intellettuale. Condividendo il pensiero dello studioso Steven Greenblatt, Mark Currie (1998) ha individuato la seguente dinamica rispetto alle forme del racconto storico nell'ambito della narrativa postmoderna:

Greenblatt argues that history has to renew itself by moving away from 'realist' assumptions about the meaning of a historical text towards a recognition that history and literature are discourses which construct rather than reflect, invent rather than discover, the past. (Currie 1998, 88)

Dunque, la narrazione storica segue un andamento che rispetta le norme stilistiche e di composizione proprie della creazione letteraria:

history carries within it the values and assumptions imposed on it by narrative exclusion and plot, so that historical knowledge often unwittingly subscribes to those values while assuming some transparent access to the past. (88)

Per comprendere questo passaggio, così da poter indagare poi meglio l'evoluzione del rapporto fra scrittura romanzesca e romanzo storico (novel) in ambito letterario, è necessario prendere le mosse da una delle codificazioni più interessanti del romance, quella operata da Northrop Frye in Anatomia della critica e nella Scrittura secolare. Come è noto Frye lega i fenomeni letterari a quelli antropologici, folclorici, più in generale ai fenomeni socio-culturali, per ricostruire strutture mitiche o archetipiche su cui si basa la narrazione. E con questa finalità cerca di interpretare le strutture ricorrenti della forma romance, partendo dall'idea che la narrazione del romance sia più strutturata di quella del novel che tende ad adeguare la propria forma alla realtà alla quale è strettamente legato.

Il punto di partenza è una forte polarizzazione tra due mondi - un mondo ideale e un mondo demoniaco - che si tramuta in termini strettamente narrativi in una opposizione conflittuale tra bene e male, tra personaggi positivi e negativi, tra buoni e cattivi: «nel romance gli eroi sono coraggiosi, le eroine bellissime, i malvagi cattivissimi, e si tiene ben poco conto delle frustrazioni, delle ambiguità, e delle difficoltà della vita comune» (Frye 1969, 151). Un altro elemento essenziale nella struttura del romance è la presenza del lieto fine, che si configura come un ritorno dell'eroe alla sua identità originaria, dopo aver superato avventure che rischiavano di trascinarlo in uno stato di alienazione e in una totale perdita di se stesso: avventure preparatorie, minori, che sono parte di un'avventura più grande che riguarda la quest dell'eroe. 
Questi fattori - eventi irreali, polarizzazione tra bene e male, lieto fine - sono dunque costitutivi del romance e sono sufficienti per costruire un ponte tra le storie sulla Shoah e il romance. Immediato è comprendere come la polarizzazione tra bene e male sia necessaria in ognuno di questi racconti che gravitano intorno alla tematica della deportazione e dello sterminio del popolo ebraico. Fortunatamente, nessuno può mettere in dubbio che i nazisti, i carnefici, rappresentino il male, la malvagità, anzi spesso diventano esempi del male assoluto. Non può e non deve esserci nessuna ambiguità su questo, neppure nei più provocatori, perciò non solo esiste questa polarizzazione ma non potrebbe non essere presente. Qualsiasi narrazione su questi temi deve prevedere questa distanza e perciò deve includere questo elemento romanzesco. Persino Primo Levi, che come vedremo nel capitolo successivo ha sempre tentato di disattivare le meccaniche romanzesche, quando introduce il concetto di 'zona grigia' nei Sommersi e i salvati, parte da questo presupposto:

In chi legge (o scrive) la storia del Lager è evidente la tendenza, anzi il bisogno, di dividere il male dal bene, di poter parteggiare, di ripetere il gesto di Cristo nel Giudizio Universale: qui i giusti, là i reprobi. (Levi [1986] 2016, 1165)

A livello puramente narratologico, possiamo facilmente individuare come nei racconti sulla Shoah i vari personaggi che operano nell'intreccio corrispondono per lo più a categorie psicologiche definite, ben riconducibili all'opposizione cardine fra 'buoni e cattivi' propria del romanzo d'avventura tradizionale o del genere fiabesco:

La caratterizzazione dei personaggi nel romance tende a seguire la struttura dialettica di questo genere letterario, il che significa che non rivelano né sottigliezza né complessità. I personaggi tendono ad essere pro o contro la ricerca. Se la favoriscono, sono idealizzati come esseri interamente puri o coraggiosi. Se la ostacolano, sono presentati quasi come caricature della loro malvagità e viltà. Quindi nel romance ogni personaggio tipico tende a trovarsi di fronte al suo opposto, in senso morale, come avviene dei pezzi bianchi e neri negli scacchi. (Frye 1978, 259)

Questa dicotomica suddivisione presente nello schema dei personaggi del romance si applica perfettamente alla caratterizzazione romanzesca delle rappresentazioni narrative della Shoah. La separazione dei figuranti in due categorie archetipiche principali aiuterebbe il narratore, secondo Frye, a non cercare di normalizzare i personaggi da lui creati; anzi, questa favorirebbe l'atteggiamento opposto, ovvero la tendenza ad estremizzare i connotati psico-caratteriali dei personaggi in due gruppi di riferimento stereotipati. Per citare diretta- 
mente la fonte: «l'autore di un romance [romancer nella versione originale] non cerca di creare delle 'persone vere' ma piuttosto delle figure stilizzate che diventano archetipi psicologici» (Frye 1978, 411).

Nel caso della Shoah, la polarizzazione dei personaggi si realizza generalmente mediante la loro suddivisione rispettivamente in 'vittime' e 'carnefici'. Poco fa abbiamo messo in evidenza come i protagonisti e gli antagonisti di moltissime delle opere incentrate sul tema della deportazione e del genocidio nazista riportino una struttura chiara e ben definita, quasi impermeabile da variazioni quando si tratta di attribuire ai personaggi dei ruoli specifici: nella fattispecie, gli ebrei e i prigionieri dei campi di concentramento ricoprono convenzionalmente il ruolo di personaggi positivi (le vittime o pedine bianche, per riprendere l'analogia con la scacchiera fatta da Frye), mentre i nazisti rappresentano la loro controparte negativa (i carnefici ossia le pedine nere). Inoltre, è possibile osservare quanto le narrazioni di finzione che trattano dell'Olocausto tendano a sviluppare i propri contenuti seguendo un'impostazione mitologica per lo sviluppo della trama - ovvero presentano un forte ascendente tragico in quanto espandono e approfondiscono le situazioni narrate insistendo sulla polarizzazione etico-psicologica.

Come Frye ha specificato, «nel romance troviamo gli elementi junghiani di libido, anima e ombra, riflessi rispettivamente nelle figure dell'eroe, dell'eroina e del malvagio» $(1978,411)$. È chiaro che la scoperta della psicanalisi e l'approfondimento dei livelli del subconscio individuale sono stati concetti fondamentali non solo per la dimensione scientifica e lo sviluppo della medicina moderna: anche la letteratura e la critica ad essa rivolta hanno ripreso, studiato e adottato molte delle teorie elaborate dalla psicanalisi per interpretare i contenuti di un certo testo o per classificare i personaggi in esso coinvolti. Nel caso del genocidio ebraico, un tale approccio investigativo ha donato un ulteriore livello di complessità e profondità ai fatti narrati dai testimoni o dai loro epigoni, compresa soprattutto la logica distorta della controparte nazista per quanto riguarda l'assurda e intransigente logica dello sterminio di massa.

Accanto alla polarizzazione dei personaggi, l'altro punto essenziale sul quale la dimensione storica della Shoah poggia nel momento in cui viene declinata in forma narrativa è il riferimento a eventi irreali e fuori dal quotidiano. Clara Reeve, già nel 1785, mette in relazione il romance con «ciò che non è mai accaduto ed è improbabile che accada» (Reeve 1785, 111 cit. in Mazzoni 2011, 95-6). Si tratta di un elemento in evidente conflitto con la memorialistica e col romanzo storico per i quali i fatti accaduti sono atrocemente reali. Però nel caso del romanzo storico si deve tener presente non tanto ciò che è reale o che non è reale, che non è in dubbio, quanto piuttosto la prossimità o meno con la nostra realtà: si tratta perciò di una questione di ricezione da parte del lettore. Se - scrive ancora Reeve - «il novel 
dà un resoconto familiare di cose come quelle che succedono ogni giorno davanti ai nostri occhi, così come possono accadere ai nostri amici e a noi» (Reeve 1785, 111 cit. in Mazzoni 2011, 95-6), si può dire che il noi contemporaneo, almeno quello occidentale, vive in un'epoca fortunatamente molto diversa da quella a ridosso della Seconda guerra mondiale e non percepisce come un rischio vicino ciò che succedeva in quell'epoca: "voi che vivete sicuri - direbbe Primo Levi - nelle vostre tiepide case» (Levi [1958] 2016, 139), segnando uno iato incolmabile tra la nostra e la loro esperienza.

In più, come si vedrà meglio più avanti e in parte s'è già accennato nel secondo capitolo di questo volume, stiamo attraversando decenni senza trauma, l'epoca dello «sciopero degli eventi» (Baudrillard 1993), che dà la sensazione di una distanza abissale dalle atrocità perpetrate in quell'epoca che paiono non solo lontane ma spesso addirittura inimmaginabili. Se il novel è quel genere letterario che riproduce la realtà quotidiana presente o passata, è evidente che i fatti del Lager, il comportamento disumano dei nazisti contro gli ebrei, le sofferenze patite dalle vittime, e in generale tutto ciò che riguarda le guerre, non possono che essere percepiti come distanti da questa quotidianità, gettati in un tempo prossimo ma lontanissimo, raggiungibile piuttosto con uno sforzo di immaginazione che grazie all'esperienza di tutti i giorni.

Non è un caso infatti che alcune delle migliori distopie traggano ispirazione proprio da quel mondo, fatto di regole rigide e astruse, ma anche di indecifrabili arbitrii e di incomparabili atrocità. Basti pensare agli universi postmoderni di Philip Dick, a cominciare ovviamente dal testo già citato The Man in the High Castle (noto in italiano col titolo banalizzante La svastica sul sole) del 1962, oppure alla società fantastica descritta da Georges Perec in $W$ o il ricordo d'infanzia del 1975, nella quale una comunità che vive su un'isola sperduta si fonda sulle regole rigide delle gare sportive, esplicitamente ispirate alla vita del Lager, come testimonia la citazione da L'universo concentrazionario di David Rousset; oppure ancora agli ironici ma terribili mondi possibili disegnati da Primo Levi in Storie naturali nel 1966. La distanza dalla quale percepiamo il mondo del Lager e le storie della Shoah è dunque paragonabile a quella con cui vediamo mondi fantastici e irreali e colmare questa distanza richiede uno sforzo che vada oltre i territori dell'umanamente pensabile: perciò fin da subito i testimoni temevano che non sarebbero stati creduti, perché il livello di disumanità raggiunto sfiora l'inimmaginabile.

Anche il terzo punto chiave del genere romanzesco, il lieto fine, sembra avere riscontro in queste narrazioni, almeno per due diverse ragioni. La prima, la più semplice, è che il nazismo, ovvero l'impero del male, ovvero narrativamente il mostro antagonista, è stato sconfitto storicamente, e questa è la premessa necessaria alla costruzione di quello che tecnicamente potrebbe chiamarsi un lieto fi- 
ne, benché questo abbia comportato la morte di milioni di innocenti. Ma ogni storia a lieto fine ha bisogno di quello che Joseph Campbell o Christofer Vogler chiamerebbero «il viaggio dell'eroe» (Vogler 2005). La semplice definizione di eroi per i sopravvissuti sembra persino offensiva. Primo Levi riteneva che soltanto i sommersi hanno vissuto fino in fondo il Lager e sarebbero loro i soli ad avere il diritto di raccontare, e la ragione è che soltanto loro non hanno una storia a lieto fine che sembra persino rendere tollerabile ogni racconto. Nel capitolo successivo, vedremo come lo scrittore torinese abbia cercato in ogni modo di togliersi di dosso l'etichetta dell'eroe, dal momento che nel Lager «sopravvivevano i peggiori» (Levi [1986] 2016, 1195), e che a coloro che scampavano la morte restava soltanto «la vergogna per essere sopravvissuti al posto di un altro» (62). Un altro espediente attraverso cui la scrittura di Levi prende le distanze dalla narrazione di finzione è proprio quello della disattivazione del lieto fine: tale meccanismo è ben visibile nell'epilogo del ritorno a casa della Tregua (1963), che diventa una delle pagine più dure dell'intera opera leviana (vedi capitolo successivo, ma cf. anche Cinquegrani 2019a), oppure anche nel finale del romanzo Se non ora, quando? (1982) nel quale alla ottimistica nascita di un bambino corrisponde immediatamente lo sgancio delle bombe atomiche su Hiroshima e Nagasaki.

Lieto fine e viaggio dell'eroe sono dunque continuamente negati dai sopravvissuti nei quali prevale il pudore o la vergogna, ma la negazione stessa è necessaria laddove la narrazione porterebbe esattamente in quella direzione. È come se gli autori più maturi, come Primo Levi, avvertissero il lettore che la storia condurrebbe all'identificazione del lettore nel protagonista eroe, che la struttura stessa della narrazione - si pensi alla mappa del viaggio che apre La tregua - porterebbe nella direzione delle più note e archetipiche strutture narrative, ma si tratta invece di una esperienza reale, una tragedia vissuta, e non di una storia di mostri ed eroi come potrebbe sembrare.

Del resto, se si affonda di più nella descrizione del romance si ritrovano altri punti di contatto con la letteratura della Shoah. Frye descrive quattro movimenti tipici del romance, che consistono nella ascesa e nella discesa in due modalità diverse. Il romance, secondo Frye, inizia spesso con una brusca discesa in un mondo inferiore. L'eroe, a causa di questa discesa, perde la propria identità, è vittima di una spaccatura rispetto alla vita precedente, questo può portare addirittura alla metamorfosi in un animale o in un vegetale - come accade al Lucio di Apuleio trasformato in asino o a Dafne trasformata in alloro. Spesso la discesa consiste nella "prigionia o paralisi o morte» (Frye 1978, 110). Altre volte, l'eroe è divorato dal mostro. L'esempio che fa Frye è quello di Giona nella parabola biblica che viene inghiottito da un enorme pesce, spesso accostato a una balena, per esserne alla fine liberato. Jona che visse nella balena è la metafora che usa ad esempio Roberto Faenza per il suo film sul Lager, che gio- 
ca ovviamente sul nome reale del protagonista, ma utilizza il richiamo alla figura biblica, perché la riconduce alla stessa struttura archetipica. Queste storie raccontano di questa discesa agli inferi, della trasformazione dell'uomo in bestia o in numero, della prigionia, del mostro che inghiotte l'eroe e poi lo risputa, nell'inevitabile lieto fine.

E proprio grazie a questa discesa agli inferi che l'eroe del romance trova la sua identità in un processo descritto bene da Jung come nekyia. Nel suo saggio su Picasso scrive per esempio: «La nekyia non è una caduta titanica, priva di scopo e meramente distruttiva, ma una significativa katabasis eis antron: una discesa nella grotta dell'iniziazione e della conoscenza misterica» (Jung 1985, 411) È in quest'ottica che Primo Levi, pur cercando di negare, come si è visto più su, ogni parabola falsamente edificante, può scrivere: «la mia vera università è stata Auschwitz» (Levi, Camon 1987), ovvero, come dice Jung, «una discesa nella grotta dell'iniziazione».

È ancora Jung che descrive questo percorso di discesa con il personaggio di Jona, in Simboli della trasformazione scrive infatti:

Allorché Giona fu ingoiato dalla balena, non si trovò semplicemente imprigionato nel ventre del mostro, ma, come racconta Paracelso, vide 'straordinari misteri' [...]. Nelle tenebre dell'inconscio è nascosto un tesoro, quello stesso 'tesoro difficile da raggiungere' che [...] viene descritto come perla luminosa o [...] come mistero, con il che si intende un 'fascinosum' per eccellenza. [...] L'eroe è un eroe proprio perché in tutte le difficoltà della vita vede la resistenza contro la meta proibita e combatte questa resistenza con tutto l'anelito che lo porta verso il tesoro difficile da raggiungere o irraggiungibile; anelito questo che paralizza o uccide l'uomo comune. (Jung 1965, 323-4)

Nonostante il pudore e il rispetto col quale si deve far ricorso al termine eroe, è innegabile che il percorso è identico a quello del prigioniero che va incontro a mille difficoltà per giungere alla salvezza che, nella visione del lettore (anche se non in quella del sopravvissuto), all'uomo ordinario sarebbe preclusa. L'eroe del romance è il portatore di valori ideali, in un mondo che non li conosce o non li apprezza. Scrive Paolo Zanotti commentando il passo di Clara Reeve su riportato: «Il romance, come sempre, è lo spazio di un'assenza, il bisogno di valori ideali che probabilmente non sono mai esistiti» $(1998,40)$. Nel mondo infernale del Lager, questi valori sono inesistenti: rappresentano i più basilari fondamenti dell'essere umano, ma ciononostante sembrano scomparsi e sembrano appannaggio esclusivo dell'eroe che tenta di restare umano contro un calcolato processo di disumanizzazione operato da figure che non possono che essere disegnate come il mondo dei malvagi.

Scrive Frye: «In ogni epoca la classe sociale o intellettuale dominante tende a proiettare i suoi ideali in una qualche forma di ro- 
mance, in cui gli eroi virtuosi e le eroine bellissime rappresentano gli ideali, e i cattivi la minaccia che ostacola l'influsso dei primi sulla società» (Frye 1957, 247). Se pure i prigionieri difficilmente si disegnino come uomini virtuosi o donne bellissime, nel confronto con l'abisso di inumana malvagità degli aguzzini, la tenacia con cui tentano di conservare un recinto di umanità li rende moralmente tenaci, l'ultimo baluardo della civiltà in un mondo destinato alla deriva. Persino loro malgrado, dunque, la percezione dell'eroismo è inevitabile, laddove combattano o almeno resistano contro un nemico molto più grande di loro e dalle fattezze mostruose e deformi.

Benché dunque i presupposti del romance si adattino facilmente a raccontare i temi della Shoah, si deve comunque riconoscere che si tratta sempre di equilibri e proporzioni con le quali romance e novel si bilanciano all'interno di un'opera letteraria (accadeva già ai tempi di Walter Scott, secondo Zanotti). È, per parafrasare Roman Jakobson e anche Brian McHale, una questione di dominante. Guido Mazzoni, nel suo Teoria del romanzo, ha descritto l'evoluzione di questo complesso rapporto nei secoli, rilevando come l'accezione dei termini e anche la misura del loro utilizzo muti in modo deciso. Il punto più avanzato a cui giunge, salvo poche eccezioni, è la narrativa di Italo Calvino che cataloga tra Le forme moderne di romance a partire da una nota di Roland Barthes sul Cavaliere inesistente:

Barthes insiste sul nesso fra romance e logica che troviamo nelle opere di Calvino e, prima di lui, nella corrente narrativa con cui Calvino è imparentato [...]. Questa narrativa immaginaria, ma dall'irrazionalità controllata, questo intreccio di romanzesco e Aufklärung, o di romanzesco e metaletteratura, è una delle periferie narrative più importanti degli ultimi secoli. (Mazzoni 2011, 350)

Proprio l'intreccio tra «romanzesco e Aufklärung» caratterizza non solo Calvino ma anche Primo Levi e in generale molta della letteratura della Shoah. Se, nelle parole di Barthes, Calvino tende a far convergere «immaginazione» e «meccanica» (Barthes 1978, 1-2), questo stesso procedimento, ovvero la ricerca della struttura nel caos, è quello messo in atto da Levi, come lui stesso riconosce nei Sommersi e i salvati:

Senza una profonda semplificazione, il mondo intorno a noi sarebbe un groviglio infinito e indefinito, che sfiderebbe la nostra capacità di orientarci e di decidere le nostre azioni. Siamo insomma costretti a ridurre il conoscibile a schema: a questo scopo tendono i mirabili strumenti che ci siamo costruiti nel corso dell'evoluzione e che sono specifici del genere umano, il linguaggio ed il pensiero concettuale. (Levi [1986] 2016, 1164) 
Al linguaggio e al pensiero concettuale si aggiungono le strutture narrative che portano le storie nei territori del romance e mettono ordine nel disordine del mondo. Benché Levi guardi sempre con consapevolezza e diffidenza a questi principi ordinatori, e più volte tenti più o meno efficaci esorcismi, il suo ricorso a queste diverse forme di Aufklärung è innegabile. Così, Carlo Tirinanzi de Medici con una perentorietà persino eccessiva può definire La tregua uno «straordinario esercizio di romanzesco» $(2018,22)$, benché il punto di partenza sia l'esperienza reale e non l'immaginazione come accade in Calvino. Nel passo di Barthes ricordato da Mazzoni, il teorico francese scrive che Calvino «pone una situazione [...] irrealistica dal punto di vista della verosimiglianza del mondo, ma solo nel dato di partenza, e poi questa situazione è sviluppata in modo implacabilmente realistico e implacabilmente logico» (Mazzoni 2011, 350).

Come si è detto più su, la percezione del lettore è quella che i personaggi - ma si dovrebbe dire le persone - di Primo Levi vivano situazioni irreali e inverosimili, benché terribilmente vere, e l'autore cerca di mettere ordine nell'informe nel quale suo malgrado si trova a vivere. Non si tratta più di «meccanica dell'immaginazione» ma di meccanica dell'informe. Questa stessa tensione, del resto, come riconosce lo stesso Mazzoni, anche richiamandosi agli studi di Massimo Rizzante, è particolarmente vivida in Kafka, col quale Levi ha un rapporto di ammirazione e spavento proprio per le affinità tra la storia immaginaria del Processo che lui stesso ha tradotto nel 1981 e la propria vicenda raccontata nei suoi libri di memorie.

Anche questa accezione o questo utilizzo moderno di romance dunque descrive bene questa letteratura.

\subsection{Enric Marco e l'industria della memoria}

Data dunque per assunta la prossimità di fondo delle narrazioni della Shoah con le strutture tipiche del romance, è necessario verificarne le conseguenze, e in particolare stabilire se questo rappresenti una risorsa o un pericolo. La risorsa è certamente rappresentata dalla immediata narrabilità di queste storie, che potrebbero risultare comprensibili ai più, di facile fruizione e perciò più immediatamente divulgabili col vantaggio di conservare viva la memoria che è esattamente ciò che i reduci si sentono giustamente in dovere di fare.

Però è anche un pericolo. Perché il romance rappresenta, proprio per la ricorrenza delle sue strutture, un distaccamento dalla realtà verso le pratiche di affabulazione. Più una storia è romance e meno è credibile perché nella percezione comune si allontana dalla realtà. È un pericolo che tutti gli scrittori o quasi, sia nel tempo della memoria che nella postmemoria, hanno presente, anche se c'è chi - penso per esempio al Tarantino di Inglorious Basterds - accoglie il roman- 
ce depotenziando consapevolmente il realismo storico. È evidente però che nel tempo della postmemoria la questione si fa più difficile, più bruciante, perché dopo i testimoni l'allontanamento dal vero storico è più facile, e l'avvicinamento al romanzo di genere rischia di condurre a una progressiva falsificazione della memoria, così come è avvenuto col western che racconta eroicamente la vicenda di uomini bianchi che sterminano le popolazioni autoctone americane.

Con molta approssimazione, potremmo affermare che la differenza principale tra memoria e postmemoria sta nel fatto che nel tempo della postmemoria sia chi scrive sia il destinatario dello scritto non hanno vissuto gli eventi tragici di cui si parla. Perciò, emblematico in questo senso, potrebbe essere il caso di Enric Marco, il grande impostore raccontato da Javier Cercas in un suo recente libro, proprio perché rappresenta la falsificazione romanzesca di chi non ha vissuto i fatti che racconta. Come è noto, Marco è stato una figura centrale della memoria e della testimonianza: è stato presidente dell'associazione Amical de Mauthausen, ha partecipato a importanti eventi pubblici, ha svolto un'infaticabile attività di testimone nelle scuole per raccontare ai ragazzi - che quegli eventi non solo non hanno vissuto, ma considerano molto distanti dalla loro esperienza - la vicenda tragica del proprio internamento nel Lager di Flössenburg. Tutto ciò è accaduto finché uno storico indipendente, Benito Bermejo, ha scoperto che Marco in realtà non era mai stato in quel Lager e che aveva sempre mentito.

Come accade per gli scrittori nel tempo della postmemoria, siamo di fronte a una persona che simula la propria esperienza che non ha vissuto e così la trasmette per lo più a studenti o lettori che a loro volta non hanno vissuto quelle esperienze. Sebbene Marco sia stato e rimanga ad oggi identificato come un impostore - ossia un individuo che ha spacciato se stesso per qualcun altro - la sua figura può essere considerata letterariamente e antropologicamente affascinante in quanto egli ha saputo rendere la propria esistenza un'invenzione a livello narrativo, identificandosi completamente con il personaggio da lui stesso creato nel mondo fenomenico. In virtù di questa connotazione specifica, nell'Impostore Javier Cercas paragona subito Enric Marco al personaggio capostipite della finzione narrativa della letteratura moderna: il don Chisciotte di Miguel de Cervantes. L'autore realizza esplicitamente questo paragone dichiarando che:

Enric è pura finzione. Non ti rendi conto? Lui è una finzione enorme, una finzione, per di più incastonata nella realtà, incarnata nella realtà. Enric è come don Chisciotte: non gli è bastato vivere una vita mediocre e a ha voluto vivere una vita alla grande; e siccome non l'aveva a portata di mano, se l'è inventata. (Cercas 2015, 28)

La sovrapposizione fra Marco e don Chisciotte è uno strumento fondamentale in termini di comprensione del testo da parte del lettore: l'au- 
tore cioè fornisce al pubblico questo potente paragone per lasciargli intuire come a volte la realtà storica dei fatti, sebbene non possa ontologicamente sostituirsi alla sfera del racconto d'invenzione, riesca ad esser molto più simile alla finzione narrativa di quanto il lettore sia disposto a vedere. Più avanti nel romanzo, l'autore torna a riflettere su questo parallelismo affermando che quella fra i due personaggi:

Fu un'identificazione completa, radicale: per comprendere Marco bisogna comprendere che, in un certo qual modo, non fingeva di essere un deportato; o che almeno non lo finse a partire da un determinato momento: a partire da un determinato momento, Marco divenne un deportato, così come, a partire da un determinato momento, Alonso Quijano divenne don Chisciotte. (255)

Sembra che finzione narrativa e dimensione del reale costituiscano due facce della stessa medaglia. È allora opportuno tenere a mente il personaggio di Marco per comprendere fino in fondo la dinamica secondo cui un testo come L'impostore e il profilo del personaggio di Enric Marco siano, ad oggi, effettivi prodotti letterari sviluppatisi da una precisa eredità storica: la memoria della Shoah si è in principio declinata nelle forme del racconto reale ad opera dei sopravvissuti per poi evolversi ed espandersi entro una dimensione letteraria che non è più solo narrazione, ma pura finzione - tanto quanto lo è stata per anni l'impostura di Marco presso le autorità spagnole e le associazioni di ex-prigionieri e deportati di età nazista.

Come è possibile tuttavia conciliare questa nuova, interessante dimensione narrativa con quella che, tradizionalmente, la storia ed i testimoni sopravvissuti alla Shoah hanno voluto tramandare alle generazioni presenti e future? Le scritture postmemoriali presentano infatti un problema etico, il quale si colloca nel rischio sempre in agguato di cadere (e far cadere il lettore) in una deriva epistemologica, ovvero in ciò che Cercas definisce «kitsch storico» $(2015,179)$ : è infatti responsabilità della voce autoriale quella di preservare il lettore dal credere o dal produrre un ricordo falso, distorto dei crimini nazisti, che verosimilmente può invece generarsi nella finzione letteraria rielaborata rispetto ai fatti storici realmente accaduti. A dispetto del valore artistico di molti prodotti postmemoriali sui campi di concentramento, il problema è insito nella loro eccessiva drammatizzazione che, per così dire, aumenta la distanza del pubblico destinatario fra i fatti descritti e la loro dimensione storica, incrementando parallelamente il rischio che questi non vengano più percepiti come drammatici in quanto realmente accaduti, ma in virtù della loro carica romanzesca.

In un capitolo decisivo dell'Impostore, il solo dichiaratamente inventato dell'intero libro, è lo stesso autore a supporre una corrispondenza o una sovrapposizione tra l'attività di scrittura e l'impostura di Marco: 
«Sa quanti giornalisti o quanti studenti venivano a trovarmi, nel 2001 o 2002 o 2003 o 2004 o 2005, credendo di aver trovato il loro Miralles, il loro soldato di tutte le guerre giuste, il loro eroe dimenticato? E io cosa avrei dovuto fare? Mandarli a quel paese? Dire che gli eroi non esistono? Cerco che no: gli davo ciò che erano venuti a cercare, che era ciò che lei gli aveva dato nel suo romanzo».

«La differenza è che Miralles era davvero un eroe, e lei no. La differenza è che Miralles non mentiva, e lei sì. La differenza è che nemmeno io mentivo».

«Come no?»

«Io mentivo con la verità, mentivo legittimamente, come si mente nei romanzi, io mi sono inventato Miralles per parlare degli eroi e dei morti, per ricordare quegli uomini dimenticati dalla storia». (Cercas 2015, 341-2)

Dunque parlare di Marco serve a Cercas per parlare anche dell'impostura della scrittura, del romanzo storico scritto da chi non ha vissuto gli eventi storici. Del resto, come già ricordato nel capitolo «Postmoderno e Shoah», la questione era affrontata anche in Soldati di Salamina proprio in relazione al romance. Nel dialogo del narratore con Miralles, nel quale il protagonista racconta la storia di Sánchez Mazas, si fa riferimento proprio alla dimensione romanzesca in contrasto con la vita realmente vissuta:

«Una storia romanzesca» disse poi, in tono neutro, prendendo una sigaretta dal pacchetto mezzo pieno del mattino.

Mi ricordai di Miquel Aguirre e dissi:

«Probabilmente sì. Ma tutte le guerre abbondano di storie romanzesche, no?»

«Solo per chi non le vive». (Cercas 2002, 198)

Il romance dunque sussiste solo - o soprattutto - per chi non vive la guerra. Perciò il problema diventa centrale tanto più nel tempo della postmemoria e perciò il caso di Enric Marco risulta particolarmente significativo. Tra Marco e i veri deportati esisteva già prima che fosse rivelata la menzogna una certa diffidenza, un taciuto sospetto, come se l'impostore fosse riconosciuto come un diverso. E quella diversità era la ragione del suo successo, che ci fu non nonostante non fosse stato davvero internato, ma proprio perché non era stato internato. Scrive Cercas che Marco:

Possedeva l'energia e la gioventù che i deportati non avevano più. Inoltre, non era parco di parole come loro o come quasi tutti loro, né così riluttante a parlare della sua esperienza nei campi; al contrario gli piaceva moltissimo farlo, e sapeva farlo o almeno sa- 
peva far venire gli occhi lucidi alla gente con i suoi racconti vissuti. In realtà, quando parlava in pubblico Marco risultava molto più convincente degli autentici deportati, e infatti quando teneva conferenze insieme a loro li eclissava, diventava l'uomo che commuoveva e affascinava il pubblico, il centro assoluto dell'evento. (Cercas 2015, 270)

Cercas fa alcuni esempi di questi episodi commoventi che decretavano il successo di Marco. Di sicuro impatto presso gli uditori, per esempio, la sua partita a scacchi con una SS, nella quale, sprezzante del pericolo, il nostro eroe non esita a battere il nemico per ripristinare la propria dignità perduta, anche a costo della vita, se necessario. Oppure l'episodio in cui durante una selezione Marco fissa negli occhi un aguzzino che sta per mandarlo a morte e gli sorride con «il sorriso più attraente che abbia mai rivolto a qualcuno» (Cercas 2015, 125) e l'SS decide per questo di risparmiarlo.

Si tratta di episodi miserabili se letti con la consapevolezza di oggi, ma, quando si credeva che fossero veri, certamente commoventi, potenti per il pubblico curioso e avido di storie di questo tipo. E il successo è dovuto proprio alla loro natura inverosimile e facilmente commovente e perciò romanzesca: l'eroe nel suo viaggio affronta il nemico, sprezzante del pericolo, e ne risulta vincitore. Marco costruisce un romance rispetto al quale i veri testimoni oppongono il pudore e la vergogna, reticenti a raccontare se non con dolore, col senso di colpa che sempre, e benché ingiustificatamente, segna profondamente il sopravvissuto. Ecco dunque la differenza, la frattura: il testimone diffida del romance che può emergere facilmente dalle storie sulla Shoah, il falso testimone si affida invece al romance per giungere al successo presso il pubblico, con una pratica che potremmo definire di sciacallaggio morale rispetto a una tragedia tra le più grandi della nostra storia.

Ma il successo di pubblico conduce a un interessamento da parte dell'industria e del marketing che a sua volta condiziona la struttura di queste storie. Lo dice in modo programmaticamente provocatorio Massimiliano Parente in Il più grande artista del mondo dopo Adolf Hitler:

La gente appena sente Hitler presta attenzione, il cervello si mette sull'attenti. Non è necessario che facciate un discorso, avvicinatevi a un passante e dite "Hitler!" e osservate le reazioni. Hitler, per un artista, è una pacchia. (Parente 2018)

È possibile giungere a questa percezione pop della tragedia nazista, che nel romanzo di Parente si chiama Nazi Pop Art e in un saggio che ne analizza le caratteristiche Pop Shoah, attraverso un processo che Cercas chiama «industria della memoria» che si ciba per lo più di finzione o delle malie dello storytelling: 
L'industria della memoria è risultata letale per la memoria, o per ciò che chiamiamo memoria e che era solo un vigliacco eufemismo. È stata forse l'ultima opportunità, e l'abbiamo persa. La cosa peggiore è credere che ci si salvi per essersi salvati: forse per anni la finzione ci ha salvati, così come per anni ha salvato Marco e Don Chisciotte; però alla fine soltanto la realtà può salvarci. (Cercas 2015, 293)

L'industria della memoria genera e si alimenta del kitsch storico:

La nuova industria della memoria ha bisogno di alimentarsi del kitsch storico, che regala a chi lo consuma l'illusione di conoscere la storia reale risparmiandogli gli sforzi, ma soprattutto risparmiandogli le ironie e le contraddizioni e i disagi e le delusioni che quella conoscenza procura. (179)

Il kitsch è strettamente legato al romanzesco, anzi si potrebbe dire che è il romanzesco che scinde le sue motivazioni dalle basi etiche o storiche che comunque dovrebbe avere. Cercas lo spiega in questi termini:

è pura menzogna (vale a dire: puro kitsch) la versione romanzesca e ornamentale della storia che Marco propalava [...], narrazioni zeppe di emozioni e colpi a effetto ed enfasi melodrammatiche, generose in sentimentalismo ma immuni dalla complessità e ambiguità della realtà, in cui il ruolo del protagonista è svolto da un eroe di cartapesta. (178)

Il kitsch dunque è una degenerazione del romanzesco che allontana dalla realtà storica, e si fa mero intrattenimento con un tranquillizzante volto morale:

Cos'è l'industria della memoria? Un affare. Cosa produce questo affare? Un surrogato, un abbassamento, una prostituzione della memoria; e anche una prostituzione e un abbassamento e un surrogato della storia, perché, in tempi di memoria, quest'ultima occupa gran parte del posto della storia. O, detto in altro modo: l'industria della memoria sta alla storia autentica come l'industria dell'intrattenimento sta all'autentica arte e, così come il kitsch estetico è il risultato dell'industria dell'intrattenimento, il kitsch storico è il risultato dell'industria della memoria. Il kitsch storico; vale a dire: la menzogna storica. (291)

Il kitsch, dunque, come versione parossistica del romance rende appetibili questi temi all'industria della memoria che è, per lo più, industria dell'intrattenimento. Nel già citato capitolo 8 della terza parte, l'autore, come si è visto, mette in scena un confronto serrato (fittizio) 
tra se stesso e Marco il cui tema principale è proprio il ruolo che i due hanno avuto nell'alimentare o approfittare dell'industria della memoria, l'uno pubblicando Soldati di Salamina il cui successo può essere ascritto anche alla moda per temi storici e l'altro ovviamente sfruttando l'attenzione per questi temi per raggiungere la notorietà. Cercas si difende in questi termini, nel prosieguo del dialogo citato più su:

«Io mentivo con la verità, mentivo legittimamente, come si mente nei romanzi, io mi sono inventato Miralles per parlare degli eroi e dei morti, per ricordare quegli uomini dimenticati dalla storia».

«E cosa ho fatto io? La stessa cosa che ha fatto lei; anzi, no: l'ho fatto molto meglio di lei. Io mi sono inventato un tipo come Miralles, solo che questo Miralles era vivo e andava nelle scuole e parlava ai ragazzi dell'orrore dei campi nazisti e degli spagnoli seppelliti lì e della giustizia e della libertà e della solidarietà [...]». (Cercas 2015, 342)

La differenza che corre tra Cercas e Marco riguarda allora soltanto lo statuto della finzione e del romanzo ovvero il medium non il messaggio, ma questa affermazione, come si sa, è estremamente ambigua:

«Tutti sanno che i romanzieri ingannano, ma nessuno sapeva che lei lo faceva. Perché l'inganno del romanziere è un inganno consentito, e il suo no. Perché il romanziere ha l'obbligo di ingannare e lei aveva l'obbligo di dire la verità. Queste sono le regole del gioco, e lei le ha infrante». (342)

Si tratta di un'affermazione rischiosa che più avanti nel dialogo verrà messa in discussione. Perché Soldati di Salamina è un libro che forza il patto col lettore presentandosi esplicitamente come «un romanzo senza finzione» benché non lo sia. Ma così facendo limita o dissimula la sua dimensione di storytelling e di romance.

Se, come abbiamo detto, in ogni romanzo sussiste una contaminazione di romance e novel pur sopravvivendo una dominante, lo stesso vale per il patto col lettore e le aspettative che in esso si generano. Il lettore che acquisterà un fantasy o un romanzo distopico si attenderà meno realtà e verosimiglianza che in un romanzo storico. Allo stesso modo un romanzo come Soldati di Salamina, il cui protagonista si chiama Javier Cercas e ha alcune caratteristiche in comune con l'autore e dichiara più volte che sta scrivendo un romanzo senza finzione, porta il lettore ad attendersi di trovarsi nei territori del novel se non proprio della testimonianza autobiografica.

Nella storia del romanzo degli ultimi decenni, come si è visto, l'epoca del postmoderno ha prodotto opere con un'accentuata dimensione romanzesca ma che nelle aspettative del lettore si presentavano esattamente come tali. Negli anni Duemila invece il lettore, a cau- 
sa di alcuni ricercati meccanismi testuali e paratestuali, è portato a credere che ciò che legge sia sempre reale o quanto meno realistico o verosimile e perciò la tecnica dello storytelling o le suggestioni del romance che comunque fanno parte di quelle scritture si configurano come un inganno o come kitsch. Per questa ragione questo tema diviene centrale nella trasmissione della memoria, della postmemoria o della storia, per i rapporti di forza che si ingenerano tra romance e patto col lettore, tra storytelling e realismo.

\subsection{Nostalgia del trauma e Reality Hunger}

All'inizio degli anni Zero in tutto il mondo occidentale escono delle opere che focalizzano l'attenzione di pubblico e critica: in Francia, nel 2000, viene pubblicato L'Avversario di Emmanuel Carrère; in Spagna nel 2001 vede la luce il già citato Soldati di Salamina di Javier Cercas; nello stesso anno, in Germania, compare il capolavoro postumo di W.G. Sebald Austerlitz; il 2002 è l'anno di Ogni cosa è illuminata, apprezzatissimo esordio dello statunitense Jonathan Safran Foer; proprio in quell'anno il regista Michael Moore porta sugli schermi Bowling for Colombine, destinato a rifondare gli equilibri tra documentario e fiction per gli anni successivi. Bisognerà invece attendere il 2006 perché in Italia si pubblichi Gomorra di Roberto Saviano, che diviene ben presto un best-seller internazionale.

Queste opere (alle quali si può aggiungere facilmente $\mathrm{HHhH}$ di Binet, già affrontato nel capitolo precedente), tutte decisive per rimarcare una soglia nello sviluppo della narrativa di tutto il mondo occidentale, hanno alcuni evidenti punti in comune, ovvero:

1. Un rapporto diretto, anche se variamente mediato, con la realtà, sia nella forma, che spesso contamina reportage, fiction e autobiografia, sia nei contenuti, che sempre si rifanno a storie vere, sia nell'ambiguità della voce narrante, che quasi sempre allude a quella dell'autore;

2. La potenza disturbante delle storie raccontate, siano quelle di un pluriomicida menzognero o della criminalità organizzata, di ragazzi stragisti o, spesso, di figure limitrofe al nazifascismo.

Questi elementi determinano una svolta nella storia letteraria recente che genera un ampio dibattito critico sulla fine del postmoderno (per cui vedi il capitolo «Postmoderno e Shoah») e sulla nascita di una nuova epoca, definita variamente con le espressioni di Postpostmodernism (Jeffrey T. Nealon), Il ritorno del Reale (formula mutuata da uno studio di Hal Foster relativo all'arte di alcuni anni prima) o, con apparente contraddizione, Deserto del Reale (Slavoj Žižek). 
Nel 2014, anche Raffaele Donnarumma ha contribuito al dibattito critico sul rapporto fra realtà e finzione letteraria, individuando proprio nelle cosiddette scritture non-fiction la svolta narrativa, retrodatata a metà degli anni Novanta, che ha sancito in maniera definitiva lo stacco dal postmodernismo in merito sia alla figura dell'autore che alla forma romanzo. Nelle contemporanee scritture non-fiction «ciò che conta», dichiara Donnarumma, «è infatti che per una materia potenzialmente saggistica (lo si intuiva già in Gomorra) questi libri adottino comunque forme narrative e, vale la pena ripetere, non romanzesche» $(2014,119)$. Questa tipologia di prodotto narrativo sembra essere cresciuta in maniera esponenziale negli ultimi anni, fino a diventare un vero e proprio genere di tendenza nel mercato editoriale.

Una tale osservazione rimanda a sua volta, necessariamente, alla riflessione più ampia su quali siano di fatto i caratteri essenziali della fiction e, per contro, dove si trovi essenzialmente il confine fra quest'ultima e la dimensione 'del reale'. Lo scarto narrativo tra fiction propriamente detta e realtà si sta riducendo sempre di più nel caso della letteratura ipermoderna descritta da Donnarumma, e questa riduzione risulta particolarmente accentuata qualora si considerino le caratteristiche riguardanti il racconto di un'esperienza o di un'eventualità storica:

Il racconto diviene lo strumento più adatto, perché il problema non è capire, ma fare esperienza: non osservare, ma essere presi. Se il postmoderno era stato segnato da una svolta linguistica, l'ipermoderno è segnato invece da una svolta narrativa.

Questi libri insomma, non sono non fiction novel perché non sono novel. [...]

Non è l'ambiguità postmoderna, che punta all'indecidibilità; è semmai un'ambivalenza ipermoderna, che mantiene la tensione. Il documento stesso, in quanto realtà già scritta, manifesta questa natura duplice. Inoltre, il documento ha un carattere performativo: esso non si limita a registrare, ma pone in essere. (Donnarumma 2104, 120, 124)

In linea con questa prospettiva d'indagine, le opere incentrate sul tema della Shoah possono rappresentare un chiaro esempio di come una precisa fattualità storica sia in grado di generare delle modalità di narrazione svincolate sia dal piano del reale che, al tempo stesso, da quello della finzione propriamente detta. Ad ogni modo, possiamo affermare che rispetto al postmoderno, cioè all'epoca in cui l'alternativa alla Storia era più allettante dello stesso presente storico, è subentrata una nuova attenzione per la realtà circostante l'individuo contemporaneo.

Le ragioni di questo cambiamento di paradigma sono state spiegate in modi diversi: c'è chi, come Žižek (2002), attribuisce un gran- 
de potere simbolico all'attentato dell'11 settembre, chi analizza la nascita e la diffusione del web (si veda, per la precocità dell'indagine, il finale di Underworld di Don DeLillo), chi addirittura aggiunge a questi elementi la trasformazione della televisione verso il reality show (Jean Baudrillard 2014). Ma al di là di singoli episodi è utile in questa sede analizzare fenomeni più generali e ampiamente studiati per comprendere e spiegare il fenomeno e che si potrebbero sintetizzare in due elementi: la nostalgia del trauma e la Reality Hunger.

Almeno a partire dal 1996, l'anno di Unclaimed Experience: Trauma, Narrative, and History di Cathy Caruth e di Worlds of Hurt: Reading the Literatures of Trauma di Kalí Tal, si è sviluppato un filone di studi di critica letteraria che è andato sotto il nome di Trauma Studies. Sia pure con le diverse prospettive dalle quali gli studiosi in questi anni hanno guardato il fenomeno, la teoria che sta alla base è relativamente semplice. L'uomo occidentale ha vissuto un lungo periodo di Fine della storia (Francis Fukuyama 1992) o di sciopero degli eventi (Jean Baudrillard 1993) e perciò di assenza di un trauma reale; questo ha comportato una sorta di inconscia nostalgia (la «nostalgia dei giorni del disordine» di cui parla DeLillo 2000, 862), compensata nelle opere letterarie e cinematografiche da un proliferare di storie forti che rievocano il trauma non vissuto (il nesso è evidente fin dal titolo del saggio di Daniele Giglioli, Senza trauma: scrittura dell'estremo e narrativa del nuovo millennio [2011]). Sono tutte indagini sul trauma quelle dei libri ricordati più su, dei libri soglia dell'inizio degli anni Zero: e certamente il trauma storico più forte, con un valore iniziatico e simbolico oltre che tragicamente reale, è, per tutto l'Occidente, quello del nazifascismo. Quindi, scrive ancora Cercas: «ai nostri tempi il testimone ha acquistato un prestigio così smisurato che nessuno si azzarda a mettere in discussione la sua autorità» $(2015,263)$.

L'altro punto nodale è la rinata attenzione per la realtà, che forse deriva da una progressiva volatilizzazione della stessa ad opera soprattutto del web, o forse da una incapacità di distinguere il Reale dal Simbolico. David Shields in un celebre e discusso libro del 2010 ha utilizzato per questo fenomeno la suggestiva espressione Reality Hunger, il cui sottotitolo, A Manifesto, ne rivela il carattere militante. Secondo l'autore la letteratura dovrebbe andare sempre più verso un'analisi della realtà in forme nuove, ibride, che superino l'egemonia del romanzo o più in generale dello storytelling. Il nemico, a questo punto, divengono le trame, che avevano un valore preponderante in epoca postmoderna.

Il libro è almeno in parte una presa d'atto di quanto già stava avvenendo da tempo nella letteratura, soprattutto in Europa, ovvero il proliferare di forme ibride tra romanzo e saggio che avessero per oggetto storie vere e non più inventate. Nella dicotomia da cui si è partiti, tra novel e romance, sembra che il novel più rigoroso trionfi. Se, con Scott, nel novel «i fatti si adattano al corso ordinario del- 
le vicende umane e al moderno assetto della società», il nuovo realismo aderisce pienamente a questa definizione.

Il problema è che l'uomo da sempre ha avuto fame di storie oltre che di realtà, e le storie hanno necessariamente bisogno di essere raccontate, di storytelling. Così, paradossalmente, più la forma letteraria si avvicina al novel rivolgendosi quasi esclusivamente a storie vere, più queste devono essere emotivamente forti e coinvolgenti, possibilmente ricche di eroi del bene o del male per soddisfare le esigenze di emozione e di catarsi del lettore. Siamo di fronte quindi a un duplice movimento: quello verso la realtà soddisfa la nuova esigenza di aderire al mondo; quello verso il romance soddisfa l'esigenza, ancora e sempre vitale, di fuga nella fantasia. Soltanto storie reali ricche di pathos e di romanzesco potranno soddisfare queste condizioni: così Carrère si rivolge alla storia quasi inverosimile di Jean-Claude Romand, Cercas inventa Miralles, Foer risale nelle vite dei propri antenati vittime dei nazisti, e così via.

In anni recenti, quindi, gli anni della postmemoria, le storie assolute, tragiche e estreme della Shoah rispondono a una esigenza dei nuovi lettori, che vi ritrovano il trauma, il reale e il romanzesco in un mix continuamente in bilico tra esigenze tra loro contraddittorie. Diventare Enric Marco, l'impostore che da storie vere ricava suggestioni posticce facendo un pessimo servizio al raggiungimento della verità, è un rischio concreto, che gli autori che guardano a queste vicende devono sempre tenere presente.

Un esempio significativo di questa ambiguità, che è affrontata con consapevolezza, è il recente e già citato La scomparsa di Josef Mengele di Olivier Guez (2018), non un libro direttamente sulla Shoah, ma certo ad essa strettamente legato, dato che parla di uno dei suoi più sinistri protagonisti, anche se nel periodo successivo alla caduta del regime nazista. Il romanzo reca in coda otto pagine di bibliografia, quasi a voler affermare l'adesione alla storiografia, ma anche, forse, a voler denunciare il senso di colpa di fare un romanzo sulla storia, un po' come accadeva, con un'altra urgenza morale, al Primo Levi di Se non ora, quando?. E all'interno del testo si citano e discutono alcune fonti, come nelle pagine in cui si riportano alcuni passi di $\mathrm{Me}$ dico ad Auschwitz di Nyiszli. Se questa è la dimensione di novel che Guez cerca, dall'altra parte non può negare che la storia abbia palesi elementi romanzeschi e così ne parla lui stesso come:

la leggenda di un supercattivo inafferrabile come Goldfinger, un'incarnazione pop del male, invincibile, ricchissimo e astuto, che semina i suoi inseguitori ed esce indenne dalle situazioni più pericolose, senza un graffio. A metà degli anni Sessanta James Bond trionfa sugli schermi e il dottor Mengele diventa un marchio che solo a nominarlo gela il sangue e gonfia le tirature di libri e riviste: l'archetipo del nazista freddo e sadico, un mostro. (Guez 2018, 139) 
Guez, a dire il vero, cerca di disattivare questa leggenda, ma pur con questo intento, non può che fare i conti col romanzesco insito in questa storia. Nel tempo della postmemoria, è necessario muoversi su questo crinale strettissimo, è necessario fare i conti con questa ambiguità. In un'epoca in cui non è più possibile scrivere storie come quelle di James Bond, è necessario vigilare affinché a compensare questa assenza non intervengano storie vere, romanzesche ma tragicamente reali, avventurose ma moralmente appaganti: perché Mengele non diventi davvero Goldfinger. 



\section{Shoah oltre il romance: Primo Levi}

Sommario 5.1 Da Cercas a Primo Levi. - 5.2 La resistenza al romanzesco. - 5.3 Genesi della memoria dell'offesa. - 5.4 Prendersi cura del passato.

\subsection{Da Cercas a Primo Levi}

Nell'ultimo capitolo de L'impostore, che si svolge all'interno del campo di concentramento di Flossenbürg, Javier Cercas cita Primo Levi a suo figlio Raül, raccontandogli dell'insegnamento più importante imparato dalla lettura del libro più noto dello scrittore e testimone italiano:

«Hai mai letto Se questo è un uomo?»

«No» rispose.

«L'ha scritto un tipo che era stato prigioniero ad Auschwitz, e racconta quello che è successo lì spiegai. «Primo Levi, si chiamava». «Mi pare di averlo già sentito».

«Certo che hai sentito parlare di lui» proseguii. «È un ottimo scrittore, e quel libro è uno dei migliori che abbia mai letto. C'è soprattutto una scena che non dimentico, o almeno non dimentico il ricordo che ne ho [...]. Levi parla delle code che i prigionieri facevano al campo all'ora di pranzo per farsi servire la minestra. E racconta che era un momento fondamentale, il più importante della giornata: se chi ti serviva la minestra affondava molto il mestolo e tirava su un po' della sostanza che c'era in fondo al paiolo, tutto andava bene; ma se non affondava il mestolo e quello che ti serviva era soltanto liquido, catastrofe. [...] Ti rendi conto? Da quando l'ho letto non sono più riuscito a servirmi una minestra, o a vedere come la servono, senza ricordami di Levi». (Cercas 2015, 399-400) 
L'autore si rivolge alla lezione di Se questo è un uomo (1958) in riferimento al giudizio morale verso il proprio protagonista, sentenziando poco più avanti nel testo, in maniera più ampia, sull'incoscienza che ha portato un individuo come Marco a fingersi vittima della tragedia più grande della storia umana:

A volte non riesco a credere alla fortuna che ho [...] Si dice che lo sport europeo per eccellenza è il calcio, ma non è vero: lo sport europeo per eccellenza è la guerra. E arrivo io e sono il primo, la prima generazione di europei che non vive una guerra. [...] E Marco tutto questo l'ha usato per rimorchiare e comparire nella foto. E la sai una cosa? La cosa peggiore è che non credo che l'abbia fatto in malafede, in realtà ne sono sicuro. Era puro egoismo. Io, io, io, io e io! Pura ignoranza, pura incoscienza. Se Marco avesse saputo davvero cosa significa tutto questo, se l'avesse capito davvero, non avrebbe mai fatto quello che ha fatto. (Cercas 2015, 400; enfasi aggiunte)

Là a Flossenbürg, «il luogo della finzione» (396), ossia dove la menzogna ha avuto origine, il romanzo 'senza finzione' di Cercas trova il suo epilogo e la sua riconciliazione - narrativa, morale e strutturale - richiamando al fianco l'eccellenza fra le testimonianze sul genocidio nazista. Nell'impossibilità di un paragone fra Levi e Marco, si traccia una linea netta fra l'autorità dei testimoni diretti e le menzogne messe in scena dal finto testimone: una tale operazione enfatizza necessariamente, e di proposito, l'illiceità della menzogna di Marco da un lato, e dall'altro l'importanza che la testimonianza storica ancora esercita sui contemporanei.

Nel romanzo di Cercas si percepisce apertamente la problematicità morale ed emozionale lasciata dalla testimonianza dei sopravvissuti al genocidio: che un'opera come L'impostore si misuri infatti con la lezione storica di Primo Levi ci riporta al problema della finzione nelle narrazioni pertinenti la Shoah - ovvero al focus centrale di ricerca in questo volume. Sappiamo che l'incidenza di elementi romanzeschi nel racconto di Marco è stata sia la causa principale del suo successo che, successivamente, l'elemento responsabile della caduta della sua impostura. Il contrasto fra il racconto di Marco con l'autenticità storica dei fatti reali ha finito per tradire l'architettura del personaggio, smascherandone definitivamente l'inganno di testimone e sopravvissuto alla Shoah.

Facendo un confronto dal punto di vista narratologico, emerge che il discrimine principale lungo il quale la scrittura dei testimoni diretti del genocidio ebraico (come Primo Levi) si distingue dal racconto dei post-testimoni (come Marco) si situa proprio nella diversa applicazione della categoria del romance da parte dei primi: sebbene ci siano diversi fattori che avvicinano i resoconti dei testimoni ad 
altri esempi di opere trattate in questo volume, esistono anche importanti differenze che, al contrario, rendono eccezionale sul piano narratologico la lezione di un testimone diretto rispetto alla maggior parte dei prodotti culturali sulla Shoah. La produzione letteraria di Primo Levi sarà quindi considerata come caso di studio in questo capitolo in virtù di alcuni specifici meccanismi di storytelling presenti nei suoi testi, oltre che per la sua notorietà di scrittore e testimone a livello internazionale.

\subsection{La resistenza al romanzesco}

Alla domanda Perché crediamo a Primo Levi? formulata da Mario Barenghi nell'omonimo saggio (2013), si trova questa risposta: «a mio avviso Primo Levi è credibile soprattutto perché, nel suo impegno conoscitivo - che pure costituisce la conditio sine qua non del suo testimoniare - i conti non gli tornano» (Barenghi 2013, 41). Questa di Barenghi è un'affermazione disarmante se pensata in riferimento alla testimonianza dello scrittore italiano più acclamato e apprezzato di tutti, non solo per il suo impegno nella difesa della memoria di Auschwitz ma soprattutto per il rilievo della sua analisi circa la realtà concentrazionaria. In che senso a Levi non tornerebbero i conti allora? Barenghi illustra il suo ragionamento partendo dalla trama di Se questo è un uomo e de La tregua per dimostrare che l'intento principale della scrittura memoriale di Levi è 'fuori' dalla dimensione educativa del racconto: «Se Levi avesse voluto scrivere un'opera essenzialmente autobiografica, il discorso sarebbe dovuto gravitare sul versante del riscatto, del recupero» (41). Concentrandosi soprattutto sul senso ultimo del racconto della prigionia e sfruttamento in campo di concentramento nei suoi libri memoriali, Levi effettivamente omette la parte di riscatto personale, cioè non indugia a descrivere o celebrare alcun sentimento di sollievo per esser riuscito a sopravvivere al Lager.

Dal punto di vista narratologico, il resoconto di Levi mantiene comunque uno livello base di tipo autobiografico, ma l'affermazione di Barenghi sopracitata diventa rilevante nel presente contesto d'indagine proprio perché, si è detto, le caratteristiche narrative dell'esperienza concentrazionaria dei sopravvissuti toccano da vicino lo sviluppo della trama tipica del romance. Se consideriamo il racconto autobiografico di Levi sul Lager alla luce delle caratteristiche compositive della scrittura romanzesca, ci rendiamo conto della presenza di meccanismi narrativi molto simili a quelli elencati nel capitolo precedente: ad esempio, nelle dinamiche di cattura, viaggio, prigionia e sfruttamento del Levi-personaggio presso il campo di concentramento di Buna-Monowitz in Se questo è un uomo, è presente lo stesso movimento narrativo di transizione da una situazione positiva ad una negativa mediante il superamento di prove o peripezie - uno 
schema dunque classico, tipico del romance fin dai tempi del romanzo ellenistico. Questa affinità fra la trama della testimonianza storica e la scrittura romanzata di tale testimonianza in Levi si percepisce con ulteriore insistenza specialmente nel caso di testi come $L a$ tregua e Se non ora, quando?: in entrambi i libri la struttura narrativa guarda palesemente al canovaccio tipico del romanzo d'avventura - e questa affinità non rappresenta una scelta affatto scontata, se teniamo presente che Se non ora, quando? è un romanzo d'invenzione a differenza della Tregua che, invece, riprende la vera storia del ritorno a casa di Levi una volta liberato Auschwitz.

Se non ora, quando? in particolare è la storia «plausibile» (Levi [1982] 2016, 673) di una banda di partigiani russi: il racconto della coraggiosa resistenza di uomini e donne sul fronte dell'Europa orientale contro i nazisti è scandito in dodici capitoli che ricoprono l'arco temporale che va dal luglio 1943 all'agosto del 1945. I topoi della battaglia, della lotta dell'oppresso contro l'oppressore e della forza della sopravvivenza dell'uomo anche nelle situazioni di umiliazione e annichilimento più totale, vibrano fortemente nelle pagine di questo romanzo - l'unico scritto da Levi e da lui dichiarato propriamente come 'romanzo', che non a caso è stato composto a distanza molti anni dai suoi libri più propriamente testimoniali.

Di questi, La tregua è sicuramente un testo che, pur trattando della liberazione dai campi di concentramento, presenta un intreccio ricco di formule e nuclei narrativi fortemente mitici e archetipici. Le disavventure dei personaggi e i pellegrinaggi percorsi da questi fra la Russia e l'Europa dell'Est, le descrizioni di luoghi lontani, che diventano suggestivo terreno di incontro con molteplici figure-guida (si ricordi il compagno Cesare, o il greco Mordo Nahum) conservano l'impronta strutturale dei mitologemi classici della narrazione romanzesca: fra questi, sono subito riconoscibili l'elemento del viaggio e del rischio di morte o di pericolo imminente da scampare, oppure ancora il tema del ritorno a casa e il ciclo di ostacoli che l'eroe deve superare per portare a compimento il proprio destino. In aggiunta, sono frequenti gli episodi del libro in cui si avverte anche una forte carica epico-tragica della narrazione - che implica di conseguenza una consistente dose di empatia da parte del lettore nei confronti del protagonista e dei suoi personaggi, aiutanti o rivali che siano. Soprattutto il mito della 'terra promessa', del rientro a casa e della nostalgia per il paese natio si attivano potentemente nella Tregua dall'inizio alla fine: molti dei dialoghi e delle descrizioni presenti nel testo sono densi di quel sentimento di avventura e amore per la scoperta che caratterizza il genere umano nel suo errare alla ricerca di una meta indefinita, che lo completi in quanto uomo al di sopra dei propri limiti - cioè in quanto eroe.

Tornando quindi all'equazione iniziale - ovvero che autobiografia e romance possono tecnicamente coincidere nel caso della scrittura 
memoriale legata alla Shoah - possiamo sostenere che anche la testimonianza di Primo Levi costituisca un possibile esempio di sovrapposizione di due generi letterari distinti (autobiografia e romance) per quanto riguarda la narrazione dell'Olocausto, e che questa sovrapposizione si manifesti a livello d'intreccio narrativo in testi come La tregua o in Se non ora, quando?, ma non limitatamente a questi: a parte infatti questi due libri, i più famosi rispetto al mito del viaggio e del ritorno in patria, anche in alcuni dei racconti leviani si percepisce la carica romanzesca della narrazione in diversi punti, come ad esempio nel caso della raccolta Il sistema periodico, in cui il mito della materia chimica ricama le avventure principali del giovane Levi, dall'infanzia fino alla maturità, forgiando il protagonista di conoscenze e verità essenziali per superare gli ostacoli della propria esistenza.

Eppure, è nel momento stesso in cui azzardiamo una simile comparazione fra biografia e romance che i conti non tornano del tutto, come avvisa Barenghi: «a Levi l'idea stessa di redenzione, di liberazione definitiva, è sostanzialmente estranea» $(2013,43)$. È vero, e i testi lo confermano. Se confrontiamo la scrittura di Levi in Se questo è un uomo o La tregua (e parzialmente anche in Se non ora, quan$d o$ ?) con le strategie narratologiche tipiche del romance letterario, vediamo che qualcosa non funziona: la magia innescata alla fine si dissolve, l'eroe torna ma non come un campione acclamato da tutti, o come figura positiva riscattata nella sua posizione esemplare dopo il superamento di numerosi ostacoli. Non c'è nessun lieto fine o morale consolatrice, nel senso standard della fiction letteraria; soprattutto - elemento forse ancora più sconvolgente - anche il profilo dell'antagonista, dopo aver letto Primo Levi, non può più rivestire completamente la sua funzione ancestrale di male assoluto.

Proviamo quindi a individuare alcuni esempi tratti direttamente dall'opera di Levi in cui i meccanismi di resistenza al romanzesco si attivano in maniera più evidente. In particolare, vorrei portare all'attenzione tre casi distinti: il ruolo del protagonista e la sua rimozione dal piedistallo dell'eroe in senso tradizionale (1), la negazione della funzione risolutiva dell'epilogo narrativo (2) e la prospettiva complessa circa il ruolo e la natura dell'antagonista (3) nella letteratura concentrazionaria di Levi:

1 Il resoconto leviano preclude lo scioglimento della vicenda in senso romanzesco in quanto 'disattiva' la dinamica del «riscatto» e del «recupero» di cui parla Barenghi (2013), nel senso di un impossibile ripristino della condizione umana originaria del personaggio protagonista: a chi sopravvive al Lager è infatti preclusa ogni forma di rivalsa della propria individualità e dignità in senso eroico. Ne I sommersi e i salvati, Levi svela chiaramente questa impossibilità romanzesca sulla natura dell'essere umano che abbia fatto ritorno da Auschwitz: «I 'salvati' del Lager non erano i migliori, i predestinati al bene, i la- 
tori di un messaggio: quanto avevo visto io dimostrava l'esatto contrario» (Levi [1986] 2016, 1195). In questa affermazione si nota un primo capovolgimento rispetto alle funzioni standard dei figuranti nel romance. Gli antagonisti potrebbero essere infatti concepiti come i 'veri' protagonisti dell'esperienza concentrazionaria dal punto di vista narratologico, gli unici eroi che 'riescono' a uscire dalla voragine infernale del Lager: il ribaltamento della scacchiera dei personaggi è esattamente ciò emerge dalle parole di Levi.

Certamente si potrebbe controbattere precisando che I sommersi e i salvati è un saggio critico e non un romanzo, e che quindi tale affermazione non è necessariamente valida nei testi narrativi su Auschwitz compilati dall'autore. Proprio in questi libri tuttavia, al contrario di quanto il lettore possa aspettarsi da quanto descritto sopra, sono presenti diversi casi di ribaltamento narratologico delle categorie programmatiche del romance. Il più evidente riguarda la caduta dell'eroe a vittima, che Levi infatti non elabora solo sul piano teorico nella sua ultima pubblicazione: a partire dal suo primo e più famoso resoconto storico, è possibile notare come l'autore annulli ogni rivendicazione morale dell'innocenza perduta dalla natura umana - che invece, fino alla fine, si dimostra e riconferma come perduta per sempre. Nel capitolo conclusivo «Storia di dieci giorni», in data 26 gennaio (cioè il giorno prima della liberazione del campo) Levi scrive che:

Noi giacevamo in un mondo di morti e di larve. L'ultima traccia di civiltà era sparita intorno a noi e dentro di noi. L'opera di bestializzazione, intrapresa dai tedeschi trionfanti, era stata portata a compimento dai tedeschi disfatti. È uomo chi uccide, è uomo chi fa o subisce ingiustizia; non è uomo chi, perso ogni ritegno, divide il letto con un cadavere. [...] Parte del nostro esistere ha sede nelle anime di chi ci accosta: ecco perché è non-umana l'esperienza di chi ha vissuto giorni in cui l'uomo è stato una cosa agli occhi dell'uomo. (Levi [1958] 2016, 276; enfasi aggiunte)

In queste righe si compie il ribaltamento dell'eroe in antieroe: in pratica, nella penultima pagina di Se questo è un uomo il narratore dichiara che il male ha vinto, uomo è chi uccide (quindi il cattivo), e non chi perisce (i buoni). La fine dell'esperienza concentrazionaria coincide con la fine della presenza della civiltà per i prigionieri, morti o vivi che siano. È vero che i russi arrivano a liberare il campo di concentramento il giorno dopo, 27 gennaio 1945 (data che infatti costituisce il termine cronologico con cui si conclude il libro), ma il narratore si rende conto (e con lui anche il lettore) che in realtà è come se fosse già troppo tardi: il male viene sconfitto quando i tedeschi hanno ormai moralmente vinto, «l'opera di bestializzazione» si è compiuta nonostante la resa della Germania. In seno a questo col- 
lasso sociale e concettuale del mondo occidentale, l'uomo che torna da un'esperienza 'non-umana' non sarà più quello di prima.

Questa impossibilità di recupero dell'identità dell'essere umano in ogni direzione futura è la prima, irrevocabile frattura fra la realtà del Lager e la sua versione romanzata. Nel saggio del 1975 Estetica e romanzo, Michail Bachtin individua nella storia della letteratura quattro forme diverse di romanzo, a seconda del genere di cronotopo a cui appartiene il testo: il critico russo sostiene ovvero che il genere romanzo si sia evoluto nel tempo in quattro tipologie specifiche, distinte in base a precise caratteristiche spazio-temporali e ricorrenze di trama, simboli e ambientazione presenti in un determinato testo. Nel caso del primo e più antico cronotopo di romanzo codificabile dalla critica letteraria (che Bachtin chiama «romanzo greco»), una delle caratteristiche fondamentali dell'intreccio è la presenza, al centro della trama degli eventi, di un personaggio protagonista identico a se stesso dall'inizio alla fine, che cioè ribadisce la propria condizione di uomo nonostante le peripezie sottopostegli dal fato (la Tyché secondo la concezione divina del mondo greco). Nella dimensione letteraria del romanzo della più antica civiltà occidentale, Bachtin individua un'essenziale funzione di natura antropologica e sociale rispetto al ruolo e al valore del personaggio protagonista:

questa originale identità con se stesso è il centro organizzativo dell'immagine dell'uomo nel romanzo greco. E non si può sminuire il significato e la particolare profondità ideologica di questo momento dell'identità umana. Grazie a questo momento il romanzo greco è legato alle profondità del folclore delle società primitive e s'impadronisce di un elemento essenziale dell'idea popolare di uomo [...]. Per quanto impoverita e denudata sia l'identità umana nel romanzo greco, in essa tuttavia si conserva un granello prezioso dell'umanità popolare ed è trasmessa la fede nell'indistruttibile potenza dell'uomo nella sua lotta con la natura e con tutte le forze inumane. (Bachtin 2001, 252-3)

Rispetto dunque al cronotopo capostipite del genere romanzo, è evidente come il nucleo primordiale del personaggio protagonista sia definitivamente andato perduto dopo l'avvento di Auschwitz: la fede nell'imperituro statuto di superiorità dell'uomo contro gli agenti esterni e soprannaturali si rivela impraticabile dall'individuo che torna dal campo di concentramento, e dunque anche la sua caratterizzazione in personaggio segue questo nuovo schema ribaltato, non quello canonico. Oltretutto, l'immagine privilegiata dell'essere umano come personaggio prediletto, leader indiscusso della fiction narrativa, crolla dal suo piedistallo: nella narrazione che gravita intorno al Lager, la prospettiva antropocentrica è annullata e privata della propria fiducia identitaria. Lo dice, dopotutto, l'autore stesso in ma- 
niera esplicita: «i personaggi di queste pagine non sono uomini. La loro umanità è sepolta, o essi l'hanno sepolta, sotto l'offesa subita o inflitta altrui» (Levi [1958] 2016, 235).

2 La censura al romanzesco del personaggio protagonista coincide anche con l'annullamento dell'happy ending nella testimonianza primoleviana. «La forma finale del romance», dichiara Frye, «è quella della ricerca portata a termine con successo» $(1969,248)$ : cioè del lieto fine. Eppure, nel caso di Levi, anche lo scioglimento della vicenda viene sottratto dallo schema canonico del finale positivo rispetto al genere romanzo. Proprio quando la vicenda sembra all'apice del suo sviluppo e pronta per un epilogo-riscatto del protagonista, l'autore incrina di proposito il senso del lieto fine in entrambi i suoi libri memoriali.

Si è in parte già visto sopra come, nel caso di Se questo è un uomo, la liberazione di Auschwitz da parte delle truppe russe non implica l'aggiunta di una nota diretta di felicità o sollievo da parte del narratore: a dir il vero, all'arrivo dei russi il narratore fa coincidere un momento descrittivo alquanto macabro. Il Levi-personaggio e il suo compagno Charles stanno appunto seppellendo un uomo di nome Sómogyi, il cui corpo è alla vista così deteriorato da non poter più esser definito umano: «l'infame tumolo di membra stecchite, la cosa Sómogyi» (Levi [1958] 2016, 277; enfasi aggiunte). La scena, di per sé è già abbastanza drammatica, si enfatizza ancora di più quando il protagonista sottolinea il dispiacere di non poter compiere un ultimo gesto simbolico di commiato di fronte al cadavere - oppure di saluto, non è specificato - gettando l'interpretazione del lettore di quest'ultima immagine conclusiva in una sorta di limbo di impressioni non precisate fra la vita e la morte, la fine e l'inizio:

I russi arrivarono mentre Charles e io portavamo Sómogyi poco lontano. Era molto leggero. Rovesciammo la barella sulla neve grigia.

Charles si tolse il berretto. A me dispiacque di non avere il berretto. (Levi [1958] 2016, 277)

Questa appena riportata è la conclusione ultima dell'intreccio narrativo in Se questo è un uomo: le poche righe che seguono sono di carattere informativo, cioè danno notizia al lettore sulla sorte dei compagni incontrati da Levi negli ultimi dieci giorni ad Auschwitz, dopo la dipartita dei tedeschi.

Nella Tregua invece, lo spessore del lieto fine abortito è più complesso, nel senso di più estraniante e non attenuato da alcuna battuta di sospensione finale (funzione che forse l'epilogo informativo di Se questo è un uomo può assolvere). La tregua propone un'inversione dell'iperbole concettuale del finale proponendo la dimensione del sogno come livello di realtà effettiva: ovvero, è presente una deformazione onirica della presunta felicità provata al rientro in patria 
da parte del protagonista, il cui sentimento di sgomento che si attiva è pari invece a quello di un incubo. Nel capitolo «Il risveglio», che a partire dal titolo trasporta il lettore nella sospensione fra sogno e realtà della narrazione, l'amara conclusione è che il lieto fine (cioè la tregua) è già avvenuto: la fine dell'incubo è, nuovamente, un incubo. Così, nelle ultime pagine, il lettore apprende che la vera tregua è propriamente il prequel dell'epilogo, e non il suo compimento finale:

i mesi or ora trascorsi, pur duri, di vagabondaggio ai margine della civiltà, ci apparivano adesso come una tregua, una parentesi di illimitata disponibilità, un dono provvidenziale ma irripetibile del destino. (Levi [1963] 2016, 469)

Una volta disteso sul letto della propria casa, nella vita reale, è il Lager che prevale al risveglio dell'antieroe tornato al suo nido domestico - cioè il campo di concentramento avanza la sua istanza di ossessiva realtà anche dopo la fine della prigionia:

È un sogno entro un altro sogno, vario nei particolari, unico nella sostanza. Sono a tavola con la famiglia, o con amici, o al lavoro o in una campagna verde: in un ambiente insomma placido e disteso, apparentemente privo di tensione e pena; eppure provo un'angoscia sottile e profonda, la sensazione definita di una minaccia che incombe. E infatti, al procedere del sogno, [...] tutto cade e si disfa intorno a me [...]. Tutto ora è volto in caos: sono solo al centro di una nulla grigio e torbido, ed ecco, io so cosa questo significa, ed anche so di averlo sempre saputo: sono in Lager, e nulla era vero all'infuori del Lager. Il resto era breve vacanza, o inganno dei sensi, sogno [...]. Ora questo sogno interno, il sogno di pace, è finito, e nel sogno esterno, che prosegue gelido, odo risuonare una voce, ben nota; una sola parola, non imperiosa, anzi breve e sommessa. Ė il comando dell'alba in Auschwitz, una parola straniera, temuta e attesa: alzarsi, «Wstawác». (470)

Come già notato da Alessandro Cinquegrani in un altro contributo, la tragica rivelazione finale ne La tregua non è un elemento di realismo aggiunto in ultimo

per completare il quadro e far capire meglio al lettore cosa prova un reduce da un'esperienza devastante come quella: si tratta al contrario di un ribaltamento radicale della parabola dell'eroe. (Cinquegrani 2019, 340)

Il finale costituisce infatti «il punto più rischioso de La tregua» (339) proprio dal punto di vista romanzesco, non di mimesi del reale: disattivando il classico lieto fine previsto dal romance, Levi imprigiona il 
lettore nel testo, obbligandolo a fare i conti con quello stesso sentimento di contrasto provato dal suo personaggio. Il protagonista, tornando a casa, capisce che non potrà mai fare vero ritorno alla condizione iniziale di armonia e umanità del principio: allo stesso modo, il lettore, da parte sua, non può assolversi nel credere che la minaccia del totalitarismo sia ormai archiviabile dalla coscienza collettiva.

È in virtù di questo dilemma, cioè dell'impossibile condizione romanzesca della propria esperienza storica, che a Levi i «conti non tornano»: riprendendo la provocazione iniziale formulata da Barenghi dunque, possiamo sostenere che l'autore obblighi anche il proprio destinatario a scendere a patti con la non risolvibilità della sua esperienza autobiografica. In effetti, rileggendo anche a distanza di anni le pagine finali de La tregua, non è possibile per il lettore sentire una chiusura compatta del cerchio narrativo. Alla fine del libro, permane il senso di una profonda ingiustizia rispetto alla sorte che attende l'eroe una volta terminato il suo percorso. In questo senso, il lettore si trova costretto ad adottare la prospettiva di Levi - cioè del testimone assoluto - di fronte alla voragine infernale di Auschwitz.

Ancora più emblematico si potrebbe considerare il finale di Se non ora, quando?, soprattutto ricordandoci che questo è un romanzo a tutti gli effetti, anche se dichiaratamente ispirato a fatti reali. Nell'ultima pagina del libro, la vicenda della resistenza partigiana russa sembra chiudersi con una simbolica scena di resurrezione: la nascita di un bambino. L'homo novus che rigenera la speranza della comunità in un futuro migliore, è descritta con toni positivi e incoraggianti:

Si udirono grida ripetute, ronzare un motore, poi silenzio. Finalmente, a giorno pieno, uscì un'infermiera dal viso allegro, reggendo un fagottino. - Maschio, maschio, - rideva. Nessuno capì, lei si volse in giro, si trovò sottomano Izu l'irsuto, e gli dette uno strattone alla barba: - Maschio, come lui! (Levi [1982] 2016, 670)

Eppure, proprio le ultime righe del testo annullano l'emozione di questa bella notizia per il lettore, sabotando il meccanismo del lieto fine con la notizia di un evento sconcertante:

Intorno ai due si raggrupparono altri medici, suore, infermiere. Si avvicinò anche Mendel, e riuscì a vedere che il giornale, costituito da un solo foglio, portava un titolo in corpo molto grande, di cui non capì il significato. Quel giornale era del martedì 7 agosto 1945, e recava la notizia della prima bomba atomica lanciata su Hiroshima. (671)

Il male non è dunque mai del tutto arginabile dalla parabola della Storia per Levi, nemmeno nel caso di una storia inventata. Come già osservato sempre da Cinquegrani, 
non si tratta in questo caso di riportare al centro del discorso la realtà esperienziale dell'autore, come accadeva ne La tregua, si tratta piuttosto di riaffermare una visione del mondo, anch'essa, ovviamente, reale, depotenziando così la parabola romanzesca in favore di una tesi. $(2019,341)$

Il lieto fine viene dunque negato perché non esiste, secondo l'autore, alcun lieto fine definitivo:

proprio perché ritorna, si comprende che il male non è sconfitto, ma si trasforma, si insinua in altre forme, proprio quando sembra di averlo superato torna a manifestarsi, riportando l'eroe al punto di partenza. (341)

È noto che il male ricopra un ruolo essenziale in ogni narrazione romanzesca, a patto che alla fine a vincere siano però sempre i buoni. La concezione del male secondo Primo Levi è invece qualcosa di più complesso, tale da resistere non solo nell'esperienza della vita reale (e quindi anche nel resoconto autobiografico di tale esperienza), ma anche nella finzione narrativa.

3 A proposito del ruolo del 'male', quindi, è opportuno esporre un'ulteriore considerazione: ad eccezione di alcuni casi in cui la letteratura ha esplorato il mito del nazismo ribaltandone la prospettiva del personaggio da antagonista a protagonista in maniera esplicita (si veda l'esempio di Littell nel capitolo successivo), il ruolo dei tedeschi come cattivi per antonomasia si può ormai considerare una costante diffusa nell'immaginario comune di tutte le narrazioni del XI secolo. Anche nei confronti di questa categoria dalla funzione apparentemente inopinabile, Primo Levi riesce invece a disorientare il suo lettore precludendogli la possibilità di formulare giudizi immediati e stereotipici sulla crudeltà dei nazisti. Prendiamo come primo esempio la figura del dottor Pannwitz in Se questo è un uomo. Nel capitolo «Esame di chimica» si apprende che:

Pannwitz è alto, magro, biondo - ha gli occhi, i capelli e il naso come tutti i tedeschi devono averli, e siede formidabilmente dietro una complicata scrivania. [...] Quando ebbe finito di scrivere, alzò gli occhi e mi guardò. Da quel giorno, io ho pensato al Doktor Pannwitz molte volte e in molti modi. Mi sono domandato quale fosse il suo intimo funzionamento di uomo; come riempisse il suo tempo all'infuori della Polimerizzazione e della coscienza indogermanica; soprattutto, quando io sono stato di nuovo un uomo libero, ho desiderato di incontrarlo ancora, e non già per vendetta, ma solo per una mia curiosità dell'anima umana. (Levi [1958] 2016, 221-2; enfasi aggiunte) 
Il protagonista non cerca di giustificare la logica perversa dei funzionari tedeschi che lavorano in Lager, ma impone attraverso la sua riflessione uno sforzo di resistenza alla disumanizzazione della figura dell'antagonista che possiamo considerare eccezionale: questo tipo di ritratto del 'cattivo' messo in scena da Levi rappresenta forse un caso unico in tutta la letteratura sui campi di concentramento. A discapito infatti di una prevedibile reazione di astio e rancore, l'autore sceglie invece di presentare la curiosità del narratore-protagonista come il più reale e immediato tipo di sentimento che regola il rapporto fra il carnefice e la sua vittima: partendo dal presupposto che, ancora una volta, i conti del narratore di fronte a tanta assurda cattiveria non tornino, anche la vendetta pertanto - tipica reazione romanzesca dell'eroe sul male - non può essere accettata come una reazione del tutto perseguibile da parte del Levi-personaggio davanti allo sguardo del dottor Pannwitz. La vendetta diventa quindi un meccanismo irrilevante, anti-romanzesco. Continua Levi:

Perché quello sguardo non corse fra due uomini; e se io sapessi spiegare a fondo la natura di quello sguardo, scambiato come attraverso la parete di vetro di un acquario tra due esseri che abitano mezzi diversi, avrei anche spiegato l'essenza della grande follia della terza Germania. (222)

La natura dei carnefici è allora più complessa da comprendere rispetto alla tipica caratterizzazione statica che si trova nel genere del romance rispetto ai figuranti antagonisti. Ne I sommersi e i salvati, Levi analizza l'eredità storica e antropologica dei carnefici considerando anzitutto il carattere di gratuità frequente della violenza nazista, osservabile in molte delle pratiche e routine quotidiane del campo di concentramento. Una tale osservazione lascia però poi spazio, poco più avanti nel testo, ad una riflessione meno puntuale e più prospettica del 'male' insito nella dottrina nazista. Nel capitolo «Violenza inutile», prima si legge:

Ora, io credo che i dodici anni hitleriani abbiano condiviso la loro violenza con molti alti spazi-tempi storici, ma che siano stati caratterizzati da una diffusa violenza inutile, fine a se stessa, volta unicamente alla creazione di dolore [...]. (Levi [1986] 2016, 1211)

E subito dopo si afferma un duplice sentimento di giudizio:

Ripensando con il senno del poi a quegli anni, che hanno devastato l'Europa ed infine la Germania stessa, ci si sente combattuti fra due giudizi: abbiamo assistito allo svolgimento razionale di un piano disumano, o ad una manifestazione (unica, per ora, nella storia, e tuttora mal spiegata) di follia collettiva? Logica inte- 
sa al male o assenza di logica? Come spesso nelle cose umane, le due alternative coesistevano. (1211; enfasi aggiunte)

Dunque l'opzione di una risposta esclusiva rispetto a un'altra, dell'autaut fra due punti, non è una scelta praticabile rispetto alle considerazioni offerte dal testimone sulla realtà concentrazionaria. Questa è infatti strutturata secondo una complessità logica fuori dalla normale gerarchia sociale delle comunità umane. Proprio per sottolineare l'eccezionalità della deriva concettuale della dottrina nazista, che applica alla realtà leggi assolute a livello teorico e dunque irrazionali nel loro risvolto pratico, Levi insiste sulla banalizzazione della figura del prigioniero nel campo di concentramento. Nel capitolo «Stereotipi» de I sommersi e i salvati, l'autore sottolinea proprio come l'immaginario collettivo del prigioniero che si ribella non sia affatto una applicazione realistica della condizione carceraria nella quale gli individui erano costretti a sopravvivere in Lager. La concezione tuttavia a cui si tende a credere, banalmente, fa parte secondo Primo Levi di un universale stereotipico, una rappresentazione finzionale - romanzesca appunto - della cattura dell'eroe in chiave narratologica:

Ancora: il concetto dell'evasione come dovere morale e come conseguenza obbligata della cattività è costantemente ribadito dalla letteratura romantica (il Conte di Montecristo!) e popolare [...] Nell'universo del cinematografo, l'eroe ingiustamente (o magari giustamente) incarcerato è sempre un personaggio positivo, tenta sempre la fuga, anche nelle circostanze meno verosimili, e il tentativo è invariabilmente coronato da successo. [...] Il prigioniero tipico è visto come un uomo integro, nel pieno possesso del suo vigore fisico e morale, che, con la forza che nasce dalla disperazione con l'ingegno aguzzato dalla necessità, si scaglia contro le barriere, le scavalca o le infrange. (Levi [1986] 2016, 1243; enfasi aggiunte)

Il prototipo del fuggiasco in chiave romantica diventa inapplicabile alla realtà dei fatti esperiti dai superstiti, e alla scrittura che di quella realtà vuole dare conto per mezzo del testimone. La logica nazista, da una parte ricca di simboli e rappresentazioni mitologiche in virtù delle quali si è cercato di correggere e migliorare il contesto nazionale del Reich e del suo popolo, ha generato un cortocircuito nel momento in cui i principi ideologici costitutivi della politica totalitaria, megalomani e irrealistici, quasi più vicini alla finzione letteraria che alla realtà storica (eliminare il cattivo, proteggere la razza, istituire il grande regno del popolo ariano), sono stati applicati per filo e per segno al tessuto sociale umano. L'elevata percentuale di stereotipi presente nell'ideologia nazista ha prodotto, coerentemente, un'immagine spesso falsa e prototipica del concetto di lavoro, o della suddivisione delle classi sociali, dell'eugenetica, delle condizioni 
socio-normative imposte ai prigionieri nei campi, dei valori patriottici e razziali consumati dalla popolazione e così via. Come può allora la testimonianza diretta da parte di chi ha toccato con mano l'orrore del Lager, e ne è poi sopravvissuto, costruirsi a livello narrativo sfruttando la stessa tendenza alla finzione che ha causato una tale catastrofe storica? D’altro canto, proprio questa abbondanza plateale del grado di finzione ideologica presente nella dottrina nazista è stata la causa del suo stesso collasso (come per Enric Marco) - ed è proprio questo il punto su cui insiste il romanziere Jonathan Littell nel suo saggio sul nazismo dal titolo Il secco e l'umido (di cui si parlerà nel capitolo dedicato a Le Benevole).

Tornando alla messa in discussione del punto di vista del carnefice, in alcune delle opere leviane successive alla narrativa sul Lager ritornano, in chiave più romanzata, alcune figure di nazisti basate sulla realtà dei fatti di Auschwitz ma, appunto, dotate di una distanza temporale e stilistica che ci permette di cogliere un'evoluzione nella scrittura di Levi per quanto riguarda il ruolo del nazista come 'cattivo'. Questa nuova fase di rielaborazione dell'universo antagonista è testimoniata anche dal folto archivio di scambi epistolari che lo scrittore torinese ha condotto con molti cittadini tedeschi, la cui origine si colloca soprattutto negli anni successivi alla prima pubblicazione e traduzione di Se questo è un uomo in Germania, nel 1961. La più celebre di queste figure di villain rivisitate è di sicuro quella del dottor Müller, presente nel racconto «Vanadio» della raccolta Il sistema periodico (1975), di cui si riporta qui un estratto significativo:

Ritrovarmi, da uomo a uomo, a fare i conti con uno degli «altri» era stato il mio desiderio più vivo e permanente del dopo-Lager. Era stato soddisfatto solo in parte dalle lettere dei miei lettori tedeschi: non mi accontentavano, quelle oneste e generiche dichiarazioni di pentimento e di solidarietà da parte di gente mai vista, [...]. L'incontro che io aspettavo, con tanta intensità da sognarlo (in tedesco) di notte, era un incontro con uno di quelli di laggiù, che avevano disposto di noi, che non ci avevano guardati negli occhi, come se noi non avessimo avuto occhi. Non per fare vedetta: non sono un Conte di Montecristo. Solo per ristabilire le misure, e per dire «dunque?». Se questo Müller era il mio Müller, non era l'antagonista perfetto, perché in qualche modo, forse solo per un momento, aveva avuto pietà [...]. Non era l'antagonista perfetto: ma, come è noto, la perfezione è delle vicende che si raccontano, non di quelle che si vivono. (Levi [1975] 2016, 1019; enfasi aggiunte)

Secondo l'autore e narratore della vicenda, apprendiamo che la perfezione di una importante componente narratologica nello schema dei personaggi - l'antagonista in questo caso - rappresenta un obiettivo 
impossibile da raggiungere nella sua interezza sul piano del reale: la presenza dell'antagonista perfetto è praticabile solo nel mondo del racconto, del romance. Il signor Müller non «era un tedesco perfetto, ma esistono tedeschi perfetti? O ebrei perfetti?», si domanda Levi. La risposta è no: «sono un'astrazione: il passaggio dal generale al particolare riserva sempre delle sorprese stimolanti» ([1975] 2016, 1020). Dunque il cattivo per antonomasia, il nazista feroce e senza pietà, è un personaggio di fatto impossibile da comporre e descrivere, nell'esperienza narrativa di Primo Levi, anche in quelle opere successive ai libri più propriamente incentrati sulla deportazione e lo sterminio degli ebrei. Nemmeno i prigionieri ebrei potevano definirsi perfetti, in fondo, o almeno così si legge nella citazione sopra riportata.

Da una tale consapevolezza, arbitraria ma equa nella distinzione dell'impossibilità di rappresentare buoni e cattivi privandoli delle sfumature che entrambe le categorie di vittime e carnefici invece conservano in quanto personaggi ispirati a individui storicamente esistiti, Levi raggiunge un compromesso nel suo materiale narrativo: l'equilibrio del resoconto leviano rifugge, da un lato, la banalizzazione e il kitsch della scrittura romanzesca, mentre dall'altro non si fa scrupoli nell'abortire al lettore ogni tentativo di risarcimento e assoluzione dal grado di responsabilità e di potenziale colpa che ogni individuo conserva, anche solo per il solo fatto di essere erede di quella stessa umanità che ha potuto concepire lo sterminio programmatico di un popolo:

Dalla provvisorietà non possiamo uscire, ecco il punto. [...] Detto altrimenti, Levi non cede alla disperazione, ma non offre alcuna catarsi. Nessuna redenzione, nessun vero risarcimento è possibile: né in forma di comprensione esauriente, che disciolga l'evento in un concetto o in una teoria, né in forma di superamento storico o esistenziale, che renda giustizia alle vittime e quiete morale ai superstiti e ai posteri. Di qui l'assenza dalle memorie leviane di qualsiasi lusinga autoreferenziale, che suggerisca o insinui tentazioni di compiutezza. La parola deve serbare intatta la sua forza performativa, l'ostensione del gesto: osserva, visitatore, medita, considera - ricorda. (Barenghi 2013, 79)

L'intento di Levi nel mettere in crisi il lettore di ogni epoca storica riesce dunque in virtù di questa specifica capacità della parola narrativa, che, senza rinunciare alla sua dose di avventura e condivisione della propria esperienza, non si completa in una dinamica di consumo della storia raccontata dal testimone. Per questo allora, grazie alle strategie narratologiche messe in atto dall'autore e considerate in questo paragrafo, è forse più facile per il pubblico di ieri e di oggi 
credere a Primo Levi rispetto ad altri scrittori di età postmemoriale, i quali certamente sono penalizzati per non esser testimoni diretti dell'evento di cui producono memoria, ma conservano il vantaggio di essere più vicini al lettore contemporaneo in termini di sensibilità culturale. Levi ci apre gli occhi senza privarci del piacere - e del dovere, in questo caso - di riscoprire il passato per distinguerlo dalla finzione, e da chi vorrà far spacciare tale finzione per accadimento reale (Enric Marco, ad esempio). Poiché la salvezza, per esser raggiunta, impone un atteggiamento di disumana rivalsa verso il prossimo secondo la logica concentrazionaria, Levi ha declassato l'eroe a figura immeritevole di un lieto fine, ha distrutto il profilo di assoluta malvagità del ruolo dell'antagonista, e infine tolto al suo pubblico ogni ingenuità di credere che incubi e derive della storia umana come lo sterminio siano capitoli conclusi, lontani e irripetibili nella nostra esperienza sociale.

Bisogna guardarsi da senno del poi e dagli stereotipi. Più in generale, bisogna guardarsi dall'errore che consiste nel giudicare epoche e luoghi lontani col metro che prevale nel qui e nell'oggi: errore tanto più difficile da evitare quanto più è grande la distanza nello spazio e nel tempo. [...] molte minacce di allora, che oggi ci sembrano evidenti, a quel tempo erano velate dall'incredulità voluta, dalla rimozione, dalle verità consolatorie generosamente scambiate ed autocatalitiche. [...] Allora? Le paure di oggi sono meno o più fondate di quelle di allora? Al futuro siamo ciechi, non meno dei nostri padri. (Levi [1986] 2016, 1252)

La stereotipizzazione della Storia, così come la sua alterazione romanzesca, diventano pericolose se confuse con la realtà. Pensare infatti che i cattivi siano stati sconfitti per sempre è, secondo Levi, una bugia: siamo ciechi tanto quanto i nostri antenati. Ed è in questo ultimo, profondo smascheramento della finzione anche come categoria cronologica, soggetta ad un metro e a una misura diversi a seconda del tempo in cui si giudica un evento storico, che la narrativa di Levi si congeda definitivamente dalla suggestione così attraente del romance nel rispetto di tutte le vittime, «i sommersi», coloro che sono stati sepolti dalla Storia, o per meglio dire offesi dalla Storia. Conservare la memoria della loro offesa è dunque l'operazione più urgente da salvaguardare, l'unica vera memoria da non lasciar eclissare rispetto al racconto di ogni altra verità o finzione storica legata alla dimensione concentrazionaria. 


\subsection{Genesi della memoria dell'offesa}

La ragione principale per cui l'opera di Levi si presenta non solo nei contenuti, ma anche nella resa linguistica e stilistica così diversa dagli altri esempi presi in considerazione in questo volume, è anzitutto di tipo cronologico: al di là del fatto che il testimone coincida con l'autore del prodotto letterario in questione, Levi scrive di un preciso evento storico in un preciso momento storico. L'Olocausto non aveva ancora trovato una propria denominazione ma era appunto presente, dentro e fuori l'autore, non in qualità di passato, ma come istanza di un presente ingombrante, tutto da definire e, soprattutto, da comprendere. In generale, il bisogno di condividere le esperienze relative alla guerra appena conclusasi, specialmente quando l'urgenza di scriverne era accesa dalla libertà appena riacquisita, è un sentimento prorompente, ugualmente condiviso dai sopravvissuti dopo il 1945: in Italia, che si tratti di membri della lotta partigiana o di ebrei scampati allo sterminio è un dato di secondaria importanza. Fra le valutazioni in merito, si ricordi la prefazione di Italo Calvino alla seconda edizione de Il sentiero dei nidi di ragno (1964), in cui lo stesso Calvino convenne nel dichiarare che «l'esplosione letteraria di quegli anni in Italia fu, prima che un fatto d'arte, un fatto fisiologico, esistenziale, collettivo» (Calvino [1964] 2002, VI). In particolare, bisogna leggere questa necessità organica e diffusa come un importante fattore che ha condizionato un'intera generazione rispetto al dovere di trasmettere memoria del più grande e recente conflitto della Storia mondiale:

L'essere usciti da un'esperienza - guerra, guerra civile - che non aveva risparmiato nessuno, stabiliva un'immediatezza di comunicazione tra lo scrittore e il suo pubblico: si era faccia a faccia, alla pari, carichi di storie da raccontare, ognuno aveva avuto la sua, ognuno aveva vissuto vite irregolari drammatiche avventurose, ci si strappava la parola di bocca. (Calvino [1964] 2002, VI)

Eppure, gli anni del dopoguerra sono stati anche considerati carichi del sentore collettivo opposto, dove le storie di resistenza e prigionia sono servite certamente da catalizzatore narrativo senza tuttavia incontrare il gusto dell'opinione pubblica. Lo stesso filone di letteratura legato specificatamente alla testimonianza della Resistenza partigiana si afferma e consolida relativamente tardi rispetto alla data che segna la fine dell'occupazione nazista e, in questo caso, non solo per ragioni circoscritte alla unica Italia: una sorta di consapevole dimenticanza cala sulle nazioni europee ancora in cenere. A buon giudizio, Levi ha interpretato e ascritto il rifiuto iniziale del proprio racconto-memoria entro l'ottica di un «rifiuto più ampio, collettivo» (Belpoliti 2000-01, 25): 
A quel tempo la gente aveva altro da fare. Aveva da trovare un lavoro. C'era ancora il razionamento; le città erano piene di rovine; c'erano ancora gli alleati che occupavano l'Italia. La gente non aveva voglia di questo, aveva voglia di altro, di ballare per esempio, di fare feste, di mettere al mondo dei figli. Un libro come questo mio e molti altri che sono nati dopo era quasi uno sgarbo, una festa guastata. $(25)^{1}$

Se Auschwitz rappresenta il fulcro della 'civiltà della memoria', si deduce che la formazione del limbo entro cui sono cadute le testimonianze dei sopravvissuti ebrei durante quei primi anni fosse inevitabile al tempo in cui la rimozione inconscia degli orrori della guerra ha costituito l'unico sentimento comune alla maggior parte degli individui, a livello sia scritto che orale: ${ }^{2}$

Gli insuccessi e le resistenze editoriali nel primo decennio del dopoguerra - che d'altronde non caratterizzano solo il panorama italiano - mettono in luce quanto sia stato accidentato il percorso che ha trasformato il racconto dello sterminio da ricordo individuale in memoria pubblica; una metamorfosi che si compie nei decenni, accompagnandosi alle evoluzioni del contesto politico, al lavoro degli storici, al faticoso districarsi dall'intreccio con le memorie di altri eventi traumatici. Nella folla di racconti disseminati lungo i primi anni della Repubblica, la specificità del destino degli ebrei si perde in un coro di tragedie e di lutti: bisognerà addirittura attendere il 1986 per avere il primo volume che raccoglie unicamente memorie di deportazione stese da ebrei. (Baldini 2012, 758)

Come noto, Primo Levi è diventato portavoce e simbolo della necessità di trasmettere alle future generazioni memoria della Shoah ben prima del 1986: anzi, la sua figura di scrittore e testimone è diventata la più famosa nello spettro del panorama italiano in un periodo alquanto precoce, nonostante il decennio in cui (anche) la sua opera prima è finita nell'oblio delle verità premature circa la realtà concentrazionaria. Il faticoso percorso che ha portato alla pubblicazione di Se questo è un uomo da parte di Einaudi nel 1958, ovvero a distanza di 9 anni dalla prima edizione De Silva del 1947, non è di certo un caso isolato nel ritardo generale del dopoguerra. Tuttavia, il libro di Levi ha incon-

1 La citazione nell'articolo di Belpoliti è tratta originariamente dall'intervista postuma all'autore a cura di Carlo Paladini, «A colloquio con Primo Levi» [5 maggio 1986], a sua volta in Sorcinelli 1987, 148-59, e ora inserita nel terzo volume (2016) delle Opere complete a cura di M. Belpoliti (667-81).

2 Sempre nell'intervista con Paladini, a Pesaro nel 1986, Levi rimarca sul fatto che spesso parlare di «queste cose» durante il lavoro era «inopportuno per alcuni», tanto che in mensa era considerato «quasi uno squilibrato» (cf. Paladini, in Sorcinelli 1987, 148). 
trato maggiore resistenza di altri testi più esplicitamente legati all'esperienza partigiana in Italia proprio perché estremamente precoce, troppo moderno verrebbe da dire, per gli anni in cui è stato scritto.

Una volta rientrato in Italia, Levi si è trovato digerire un passato più che mai presente negli anni in cui scriveva il suo primo libro, e grazie alla sua particolare dimensione narrativa e abilità linguistica, ha oltretutto creato un canone primo rispetto alla materia letteraria di cui oggi si continua a leggere e narrare in forme diverse di fiction. Eppure, è corretto affermare che anche Levi - anzi Levi per primo - sceglie e definisce la forma del resoconto storico romanzato per raccontare la realtà concentrazionaria, coniando cioè un tipo di narrazione adatta ad esporre i propri contenuti che si trattiene dalla deriva della finzione presente nel romance (vedi § 5.2). Sostenere che i suoi libri relativi alla memoria della Shoah siano solo testimonianza, o solo narrativa, è chiaramente un giudizio parziale ed improprio. Emerge dalle stesse pagine e dagli stessi aneddoti una dimensione estremamente fluida del narrato, una chiarezza e un coinvolgimento emotivo alla vicenda nonostante l'autore non ceda al romanzesco dalle forme più pure e propriamente intese del canone letterario.

Si riprenda brevemente in mano la categoria dei personaggi facendo riferimento alle differenze fra letteratura memoriale e postmemoriale: è infatti presente una profonda frattura fra i personaggi ai quali Levi ha attribuito le storie e le peripezie svoltesi nell'universo concentrazionario ed Enric Marco. Nel caso dell'Impostore, Marco è accostato e descritto per tutto il romanzo come un audace don Chisciotte della realtà fattuale, un bugiardo che ha osato romanzare la dimensione fenomenica senza tener conto che questa operazione è lecita soltanto all'interno della dimensione narrativa. Marco è la nemesi dunque, in un certo senso, di tanti antieroi leviani - come ad esempio i compagni citati ne La tregua - di tutti i salvati ma anche di tutti i sommersi a cui Levi ha cercato di dare una voce attraverso la pagina scritta. Proprio in virtù del loro carattere di testimoni totali della ferocia nazista, i personaggi di Levi hanno bisogno di un autore che, in quanto superstite anche nel senso di testimone (super-testes), potesse restituire loro voce attraverso la dimensione letteraria: queste figure, che costituiscono testimoni reali ma anche personaggi fittizi in quanto calati in un testo narrativo, agiscono secondo le regole e le convenzioni narratologiche senza tuttavia 'esagerare' a livello di finzione. Non sono personaggi estremi - figure kitsch, per intenderci: debordano semmai a livello di impatto morale sul lettore (in quanto esempi di esseri umani trattati non da esseri umani) ma non aderiscono del tutto alle pratiche di affabulazione peculiari del romance, descritte in precedenza in questo volume. Abbiamo dimostrato come il loro statuto di personaggio sussista in virtù della dimensione narrativa offertagli da Primo Levi senza il supporto dello stereotipo dell'eroe positivo, o del perfido nazista. 
Nonostante il principio auto-regolatore della scrittura memoriale di Levi, dove le principali categorie del romance vengono o disattivate o capovolte, il lettore riesce comunque a percepire l'efficacia narrativa del personaggio leviano: la dimensione finzionale del personaggio deborda infatti oltre la pagina scritta, e questo accade perché la forma-romanzo di Levi è anche testimonianza - è cioè narrazione giustificata in virtù della trasmissione degli eventi storici. Senza questa necessità della scrittura, Cesare e il Greco Mordo Nahum de La tregua non sarebbero mai stati probabilmente i personaggi di un libro. Non hanno infatti 'potenzialità' narrativa fuori dalla testimonianza storica, ovvero non sono esempi di straordinario umano che dunque sono stati selezionati appositamente dall'autore per sfondare la barriera fra realtà e finzione, diventando campioni letterari di romance - al contrario di Enric Marco. Il personaggio protagonista della post-memoria è a tutti gli effetti un post-personaggio, in questo orizzonte di senso: è un erede, un epigono sul piano sia generazionale che narrativo rispetto a tutti i tipi umani presentati da Primo Levi.

Si abbandoni ora il focus dal personaggio nella scrittura memoriale di Primo Levi per concentrarsi sul messaggio che questo personaggio veicola, ovvero rispetto alla precisa funzione di veicolo della memoria che il figurante deve essenzialmente trasmettere al lettore. Nella prefazione di Tzvetan Todorov a $I$ sommersi e i salvati ${ }^{3}$ si legge che «la semplice memoria del male non è [dunque] sufficiente a prevenirne il ritorno; bisogna che il richiamo sia sempre accompagnato da un'interpretazione e da istruzioni per l'uso» (Todorov 2007, IX). Se la finalità cardine della testimonianza di Levi è la prevenzione del ritorno, la memoria individuale e la memoria collettiva della Shoah devono necessariamente confluire in una dimensione storica della memoria che protegga l'essere umano dal possibile 'ritorno' del male. E per farlo è necessario seguire attentamente le «istruzioni per l'uso» di cui parla Todorov. Ma cosa vuol dire?

Abbiamo visto che Levi è dovuto scendere a compromessi con la materia narrata, trattando il suo resoconto secondo uno schema canonico dal punto di vista compositivo (abbiamo cioè un inizio, un epilogo, uno sviluppo e un intreccio denso di personaggi) ma adeguan-

3 Il contributo di Todorov è apparso parzialmente per la prima volta col titolo «Primo Levi. La memoria del male non ne esclude il ritorno» sul quotidiano La Repubblica il 6 aprile 2007, a pagina 48. Oggi il testo, oltre che come prefazione nell'edizione Einaudi Super ET del 2007, è stato antologizzato nella raccolta Gli altri vivono in noi, $e$ noi viviamo in loro. Saggi 1983-2008 dell'autore, edita da Garzanti (2011), con il titolo «Il testamento di Primo Levi». 
do i contenuti ai vincoli imposti dall'oggettività e imparzialità storica della sua prospettiva di testimone, al fine di renderne lecito un giudizio morale: occorreva un modello letterario più complesso, superiore per così dire, affinché la 'favola capovolta' del genocidio nazista potesse trasmettere una sua morale autentica e duratura, non soltanto orrore - o peggio ancora, intrattenimento. In questo senso, si è già sottolineato come l'affabulazione nella scrittura di Primo Levi attragga il destinatario per poi respingerlo, compromettendo il suo ruolo di lettore davanti al fatto che egli stia leggendo non solo per piacere, ma soprattutto per dovere: il dovere di non dimenticare. Prima ancora di capire dunque, è importante stabilire in che modo farlo al fine di non cadere nella giustificazione dell'evento in sé o di alcune parabole relative a questo. A tal proposito, si ricordi che le tesi negazioniste prendono piede già dagli anni Settanta sia in Europa che negli Stati Uniti, ancora in pieno clima di elaborazione del lutto del genocidio ebraico dalla maggior parte di superstiti e spettatori della Seconda guerra mondiale. Nel tentativo di dare memoria degli eventi trascorsi, scrivendone per i presenti e per i posteri, è stato importante capire quali fossero le regole che gestiscono non solo la narrazione di finzione, ma anche la memoria, specialmente quella memoria con valore documentario che stava emergendo dalle testimonianze di coloro che erano scampati al 'grande esperimento' del Lager.

Il termine memoria è un concetto che si lega spontaneamente alla dimensione del ricordo (non a caso in inglese 'ricordo' e 'memoria' sono lessemi omonimi): la memoria si associa quasi per istinto all'esperienza del singolo, mettendolo in relazione con un passato individuale o condiviso di cui si è registrato un episodio - il quale può essere dunque riproposto in qualsiasi momento del presente se richiamato, appunto, alla 'memoria'. In questo volume si è già parlato di postmemoria, ma negli anni in cui Levi racconta e per tutto il XX secolo il concetto cardine di memoria ha incontrato ostacoli nell'essere definito: come dobbiamo intendere la memoria, in primo luogo? Come facoltà propria dell'inconscio o consapevole, parte dell'intelletto umano? E in quale misura si relaziona la memoria poi con gli eventi e la dimensione sociale esterna al singolo individuo?

Il concetto di memoria collettiva è relativamente recente: è stato coniato dal sociologo francese Maurice Halbwachs durante la prima metà del Novecento. Partendo da Henri Bergson e dalla dualità dei concetti di «memoria-abitudine» e «memoria-immagine» (cf. Bergson 1996), Halbwachs sviluppa la sua analisi moltiplicando e ribaltando la prospettiva bergsoniana della memoria, aprendola cioè alla possibilità che si tratti di un concetto collettivo, plurimo e condiviso prima che identitario o individuale. Questa ricollocazione della memoria rimbalza necessariamente verso un'altra problematica, che è quella della collocazione dell'individuo in quanto partecipe di un determinato tempo sociale e dunque di una specifica memoria 'collettiva'. 
Secondo Halbwachs, ricordare coincide infatti con l'atto di produrre una memoria 'di gruppo'.

I nostri ricordi vivono in noi come ricordi collettivi, e ci sono rammentati dagli altri, anche quando si tratta di avvenimenti in cui siamo stati coinvolti solo noi, e di oggetti che solo noi abbiamo visto. Il fatto è che, in realtà, non siamo mai soli. Non è necessario che altri siano presenti, che si distinguano materialmente da noi: perché ciascuno di noi porta sempre con sé e dentro di sé una quantità di persone distinte. (Halbwachs 1987, 38; enfasi aggiunte)

Se l'atto del ricordare deve essere ricollocato entro i parametri di un punto di pensiero collettivo e non individuale, bisogna allora precisare che anche storia e memoria, o per meglio dire memoria storica e memoria collettiva, rimangono due concetti ben distinti fra loro. La storia è distinta dalla memoria in quanto quest'ultima - essendo collettiva - è valida per un gruppo ed entro i limiti del gruppo stesso: vale a dire che quando il gruppo si smembra, quando i suoi rappresentanti muoiono o, come i testimoni dell'Olocausto, non condividono più gli stessi ricordi con un altro gruppo (che ad esempio può essere la generazione successiva), ecco che quel tipo di materiale memoriale diventa inutile, cioè si auto-esaurisce, non ha più lo stesso valore. Invece, a differenza della memoria:

La storia, che si colloca al di fuori e al di sopra dei gruppi umani, non esita ad introdurre nella corrente dei fatti delle divisioni semplici, il cui posto è fissato una volta per tutte. Così facendo, obbedisce ad un bisogno didattico di schematizzazione. [...] Visti da lontano e tutti insieme, ma soprattutto visti dall'esterno, contemplati da uno spettatore che non fa parte dei gruppi che osserva, i fatti si lasciano raggruppare così in insiemi successivi e separati, dove ogni periodo ha un inizio, un centro e una fine. (Halbwachs 1987, 90)

Anche Primo Levi conviene nel vedere nella Storia una tendenza inesauribile ed irrefrenabile allo 'schema' di cui parla Halbwachs. Nel secondo capitolo de I sommersi e i salvati, all'esordio del cuore filosofico dell'esperienza letteraria di Levi che consiste nell'individuazione e definizione della famosa zona grigia, si legge:

Siamo stati capaci, noi reduci, di comprendere e far comprendere la nostra esperienza? Ciò che comunemente intendiamo per «comprendere» coincide con «semplificare»: senza una profonda semplificazione, il mondo intorno a noi sarebbe un groviglio infinito e indefinito [...]. Siamo insomma costretti a ridurre il conoscibile a schema [...]. Tendiamo a semplificare anche la storia [...]. La storia popolare, ed anche la storia quale viene tradizionalmente in- 
segnata nelle scuole, risente di questa tendenza manichea che rifugge dalle mezze tinte e dalle complessità: è incline a ridurre il fiume degli accadimenti umani a conflitti, e i conflitti a duelli, noi e loro, gli ateniesi e gli spartani, i romani e i cartaginesi. [...] Questo desiderio di semplificazione è giustificato, la semplificazione non sempre lo è. [...] In chi legge (o scrive) oggi la storia dei Lager è evidente la tendenza, anzi il bisogno, di dividere il male dal bene. (Levi [1986] 2016, 1164; enfasi aggiunte)

L'istinto irrefrenabile delle rappresentazioni narrative di dividere il male dal bene può sembrare apparentemente un atto immediato e legittimo, soprattutto in un contesto dove un popolo ha agito razionalmente nella spregevole direzione dello sterminio di un altro popolo: l'eccezionalità del Levi-scrittore sta invece nel mettere in guardia il suo lettore da tale semplificazione, poiché anche se parziale - dato che in quanto superstite Levi non è un sommerso - la sua 'incompleta' esperienza del Lager è comunque più complessa da risolvere per poter essere sciolta nello spartiacque dei 'buoni e cattivi', come in un film western. Meglio parlare, piuttosto che tacere: tuttavia è preferibile scegliere di testimoniare tenendo conto dei limiti e delle zone d'ombra che velano ancora sulla storia dei campi di concentramento, su quella zona grigia che rende impossibile emettere una sentenza da parte delle comunità che si appelli agli estremi concettuali di giusto e sbagliato, positivo e negativo, figli dell'ormai estinta Europa post-illuministica.

«La memoria umana è uno strumento meraviglioso ma fallace» e questa è «una verità logora» ([1986] 2016, 1155), secondo Primo Levi. I nostri ricordi non sono affidabili di volta in volta che vengono riproposti in un secondo momento, e dunque la complessità dei resoconti di vittime e carnefici passati attraverso l'universo concentrazionario viene ulteriormente a complicarsi. «La verità sui Lager è venuta alla luce attraverso una strada lunga ed una porta stretta» ([1986] 2016, 1152) dichiara Levi, sottoposta a processi di rimozione fisica dei testimoni verso la fine del 1944 o a dinamiche di rimozione psicologica da parte dei sopravvissuti di entrambe le parti, poiché il passato era troppo pesante, troppo presente.

Prima di diventare postmemoria, la memoria individuale è passata per il vincolo della memoria collettiva, agente e responsabile della trasmissione di una memoria storica specifica secondo Primo Levi, che lui stesso ha definito memoria dell'offesa, concetto fondamentale che infatti presta il titolo al primo capitolo de I sommersi e i salvati ([1986] 2016, 1155). Dal punto di vista di Levi, la memoria dell'offesa si dirama in due direzioni: quella di chi ha offeso e quella di chi è stato offeso. Attenzione però: è necessario ricordare che la caratteristica principale che incombe su entrambi i canali di sviluppo della memoria dell'offesa è la voragine concentrazionaria, ossia il Lager. Il Lager 
altera infatti la memoria fino al punto in cui, sostiene Levi, «ci troviamo davanti ad una paradossale analogia tra vittima ed oppressore» ([1986] 2016, 1156). Sono entrambi nella «stessa trappola», anche se per motivi così diversi. Chi ha offeso mente, e «nell'atto in cui mente è un attore totalmente fuso con il suo personaggio» (1156): il che assomiglia proprio a quello che ha fatto Enric Marco per tutta la sua vita.

La possibilità di una sovrapposizione fra il criminale nazista e il personaggio di Cercas, per quanto allettante, tuttavia salta: Marco è di nuovo una categoria altra, il suo ruolo si colloca oltre la memoria dell'offesa e rientra a pieno titolo nella sfera della postmemoria, non a caso. Mentre si vedrà più avanti che il post-personaggio di Maximilien Aue nelle Benevole di Littell (2007) fa ancora parte della squadra degli antagonisti senza sfumature, l'impostore di Cercas si comporta invece come uno dei carnefici proprio quando, in realtà, aveva scelto di prendere le parti dell'offeso, cioè della vittima, nell'universo ribaltato del Lager. Si tenga presente che, al tempo in cui Primo Levi rientrò in Italia dopo numerose peripezie, la vittima non era ancora considerata come una figura eroica. I due ruoli non potevano coincidere in alcun modo, e infatti non abbiamo riportato esempi di questo 'successo' nei libri testimoniali di Primo Levi, ma dimostrato l'atteggiamento contrario: nessuno dei salvati, né tanto meno qualcuno fra i sommersi di cui possiamo leggere nei racconti dei testimoni, gode del privilegio di essere un personaggio vincente, l'eroe della finzione narrativa. La spietata realtà dei fatti l'ha punito ancora prima che diventasse personaggio, e per questo motivo ogni chiave narrativa e strategia di scrittura crolla di fronte al precipizio storico di Auschwitz.

La produzione letteraria di Primo Levi sull'Olocausto si potrebbe definire come un caso di memoria anti-narrativa, nel senso di anti-romanzesca, in quanto il Lager non rifiuta soltanto la memoria, ma anche le modalità di trasmissione di questa. Tale rifiuto sembra esser valido oltre il tempo in cui ha scritto Levi, si è propagato anzi in ogni epoca storica successiva proprio per il fatto che, ancora, la letteratura tenta e crea strategie sempre nuove di narrazione e spettacolarizzazione in riferimento alla Shoah. La memoria dell'offesa è dunque una memoria anti-narrativa perché non consente di tracciare linee di demarcazione: non c'è un personaggio buono, un cattivo o un aiutante, né una situazione di evoluzione da un polo positivo ad uno negativo o viceversa. Niente è completamente bianco e nero, quando riguarda l'Olocausto, e qualora si voglia scrivere di o entro le coordinate di tale evento, le stesse tinte narrative - gli eccessi, il favoloso, il kitsch - si spengono. Il colore che infatti, non a caso, sceglie Levi è il grigio: un neutro che fissa gli estremi e li unisce in una linea dove giusto e sbagliato, vincitori o vinti non trovano un lieto fine in entrambi i sensi.

Il linguaggio della prosa leviana resiste anche in età postmemoriale, rimanendo l'elemento più emblematico, innovativo e calzante per 
esprimere una tinta che non c'è, che si rileva solo se contrapposta a colori più vivaci. In linea con Halbwachs, il quale sostiene che la memoria è decisamente collettiva anche e solo per via del fatto che un evento o un ricordo siano registrati in una determinata lingua che il singolo condivide con un determinato gruppo di simili (i quali come lui appartengono alla stessa cultura e allo stesso ceppo linguistico, nella maggior parte dei casi), così la memoria dell'offesa, sebbene abbracci una collettività di individui che si estende dalla vittima al carnefice senza eccezioni, non possiede alcun linguaggio comune passato alla storia come koinè dello sterminio: e questo è possibile in virtù del carattere disumano della realtà concentrazionaria, la quale rimane appunto indicibile anche a distanza di oltre settant'anni. Di conseguenza, anche la lingua narrativa di Levi diventa, con il suo plurilinguismo dei fatti descritti nella cornice narrativa dell'anti-romanzesco, grigia in quanto molteplice, polifonica. Se questo è un uomo non è un romanzo scritto e pensato solo per un pubblico italiano, ma veicola un messaggio universale: l'italiano è solamente la lingua che permette ad altri idiomi di trasparire e colorare la babele infernale del KZ - il Konzentrationslager.

\subsection{Prendersi cura del passato}

Memoria, linguaggio e scrittura finzionale si ibridano delle stesse contraddizioni presenti nelle logiche irrazionali a cui 'le squadre speciali' erano costrette ad obbedire all'interno del campo: è un passato imperfetto, continuamente scolorito che in un certo senso è vero, come scrive Cercas citando Faulkner, non passa mai. Ernst Nolte - celebre storico al centro del dibattito sulla valutazione scientifica dei crimini nazisti nel secondo Novecento - ha scritto e pensato qualcosa di simile allo scrittore americano, ma relativamente alla Germania nazista e al fenomeno di Auschwitz (e che proprio per questo fece scalpore e destò le critiche della comunità accademica negli anni Ottanta). Paragonando, o meglio accostando l'esperienza del Gulag bolscevico a quella del Lager nazista, il neo-revisionismo di cui Nolte è stato esponente ha cercato di guardare al passato in una prospettiva dialogica, provando a metterne in luce i caratteri dialettici in un momento storico in cui l'unicità della soluzione finale impediva un'assoluzione, un superamento del passato in virtù non tanto di un risarcimento, ma di una condanna costruttiva, che portasse ad una effettiva elaborazione del trauma del genocidio attraverso l'appropriazione di quel passato così scomodo da raccontare.

Nonostante le critiche che tale teoria ha sollevato (specie da parte del filosofo Habermas), bisogna riconoscere a Nolte di aver riflettuto in modo diverso dai propri contemporanei proprio rispetto a quel sentimento di «passato che non vuol passare» di cui si è accennato 
sia in relazione alla letteratura della memoria che della postmemoria: anzi, forse la rinnovata immanenza di quel passato e la persistenza del grigiore attorno alla morale occidentale sulla valutazione del male sono i due motivi principali per cui la postmemoria è oggi autorizzata in primo luogo, a distanza di anni dalla fine della Seconda guerra mondiale, a narrare ancora di ciò che non vuol esser narrato, ma su cui la letteratura continua a sentire la necessità di riflettere in quanto passato non del tutto 'passato', cioè in quanto presente. Si riporta l'incipit dell'articolo di Nolte, apparso per la prima volta sulla rivista Frankfurter Allgemeine Zeitung, il 6 giugno 1986:

Con «passato che non vuol passare» si può intendere soltanto il passato nazionalsocialista dei tedeschi o della Germania. Il tema implica la tesi che ogni passato di solito passa, e che in questo non passare c'è qualcosa di affatto eccezionale. D'altra parte il normale passare del passato non va inteso come scomparsa. Nei libri di storia si continua a discutere dell'età napoleonica o della classicità augustea; ma questi hanno perso, ovviamente, l'urgenza che avevano per i contemporanei, e proprio per questo possono essere affidati agli storici. Invece, a quanto pare, il passato nazionalsocialista (come ha rilevato di recente Hermann Lübbe) non soggiace a questo processo di dissoluzione e di indebolimento, ma sembra, al contrario, diventare sempre più vivo e vigoroso: non come modello bensì come spauracchio, come passato che si pone come presente, o che pende sul presente come una mannaia. (Nolte, Rusconi 1987, 3)

Facendo emergere in primo luogo quella resistenza all'archiviazione e alla schematizzazione per quanto riguarda gli eventi legati all'Olocausto, Nolte come Levi nota l'eccezionale carattere e la portata di significazione dell'eredità dei crimini perpetrati dal Terzo Reich nella parabola della storia occidentale - che sicuramente con Auschwitz ha toccato il suo punto più basso. Questo passato sembra non avere ancora trovato un modo per essere digerito, e forse il romanzo propriamente detto non è il canale più adatto per tentare sia un processo di metabolizzazione morale che di testimonianza in quanto rievocazione perenne dell'evento in sé. La memoria dell'offesa, le cui coordinate pratiche sul piano narrativo rifuggono lo sviluppo lineare e l'andamento standard della narrazione finzionale, esclude il romance in quanto potenziale veicolo di kitsch, nemico per eccellenza della verità (che invece risulta essere il canale prediletto su cui Enric Marco ha costruito la sua menzogna).

Cercas afferma che «ogni enfasi è una forma di occultamento, o di inganno» $(2015,230)$ e l'umanità non poteva permettersi di essere ingannata rispetto ai fatti di Auschwitz, rispetto al numero di persone uccise (13 al minuto, secondo l'SS Maximilien Aue di Littell), 
o ancora rispetto alle ragioni per cui il passato non passa e la zona grigia scolorisce i racconti e le testimonianze di coloro che hanno visto, mentre ha già sommerso quelle di tutte le vittime della guerra. Quale giusto mezzo adottare allora, in senso aristotelico: qual è la soluzione finale per raccontare della soluzione finale, cioè lo sterminio di migliaia di persone? Nel primo racconto della raccolta Storie naturali, pubblicata da Levi sotto pseudonimo nel 1966, un giovane medico va a trovare un suo collega anziano e ormai isolato dal mondo, che ha avuto come unico merito quello di aver sintetizzato le sensazioni olfattive in composti chimici in grado di richiamare alla memoria eventi legati al singolo individuo. I «mnemagoghi» o suscitatori di memoria, sono ricordi da annusare, sensazioni in provetta a disposizione di uso e consumo di chi non vuole lasciar passare il passato. Il vecchio dottor Montesanto, ossia l'inventore dei mnemagoghi, afferma che:

C'è chi non si cura del passato, e lascia che i morti seppelliscano i loro morti. C'è chi, invece, del passato è sollecito, e si rattrista del suo continuo svanire. C'è ancora chi ha la diligenza di tenere un diario, giorno per giorno, affinché ogni cosa sia salvata dall'oblio e chi conserva nella sua casa ricordi materializzati [...]. Io, per mia natura, non posso pensare che con orrore all'eventualità che anche solo uno dei miei ricordi abbia a cancellarsi, ed ho adottato tutti questi metodi, ma ne ho anche creato uno nuovo. [...] ho ricostruito, con esattezza e in forma conservabile, un certo numero di sensazioni che per me significano qualcosa. (Levi [1966] 2016, 484)

Curarsi del passato è dunque un dovere essenziale dell'uomo, non solo del romanziere o dello storico. La postmemoria si cura di quel passato che, come ha detto Nolte, pende sul presente "come una mannaia», e ne indaga gli effetti che hanno provocato fenomeni meta-narrativi e personaggi iper-romanzeschi, come nei casi di Cercas e Littell. Anche Primo Levi allora, in quanto scrittore antenato di tutti gli altri scrittori di età postmemoriale citati in questo volume, si è spinto a ipotizzare una conservazione della memoria dell'offesa qualora questa dovesse svanire, essere ingerita dal grigio dell'indifferenza e dallo scorrere del tempo, attraverso l'uso della fiction: i mnemagoghi sono una finzione letteraria, non rielaborazione scritta di uno strumento reale. L'immagine qui suggerita da Levi, ovvero l'olfatto che diventa la categoria sensoriale alla quale affidarci per richiamare, suscitare alla mente la memoria di ciò che per noi 'significa qualcosa', guarda già forse alla fase della postmemoria, in un certo senso, cioè alla successiva generazione di coloro che vorranno raccontare qualcosa che non hanno visto (o meglio dire annusato?) ad un pubblico a loro contemporaneo. 
Questo dei mnemagoghi rimane tuttavia un caso a parte, diverso dalla responsabilità storica e sociale che, necessariamente, si è sviluppata in molti dei pochi individui che hanno fatto ritorno da Auschwitz. «Coloro che hanno sperimentato la prigionia [...], si dividono in due categorie ben distinte, con rare sfumature intermedie: quelli che tacciono e quelli che raccontano» (Levi [1986] 2016, 1241): rispetto a quelli che non si curano del passato, o a coloro che non riescono a parlare perché l'atto del ricordo è troppo doloroso da sopportare, Levi ha scelto di agire in direzione opposta. Con la diligenza di chi tiene un diario depurandolo al tempo stesso di quelle sfumature troppo personali, ha riscostruito la sua esperienza del Lager con «esattezza e in forma conservabile» ([1966] 2016, 484), proprio come i suscitatori di memorie del dottor Montesanto. La grande beffa e offesa dell'uomo verso l'uomo, e della storia verso la categoria stessa di storia, resta accessibile alla posterità nella forma di un resoconto storico che è molto più di un semplice documento: la scrittura memoriale di Levi è letteratura, è storia, è romanzo, e soprattutto, è memoria di fatti e di «sensazioni che per me [l'autore] significano qualcosa» (484). Qualcosa di cui tutti, testimoni e post-testimoni, dobbiamo prenderci cura. 


\section{Il caso Littell}

Sommario 6.1 Auschwitz: l'aporia dell'indicibile. - 6.2 La figura del testimone e l'età dell'inesperienza. - 6.3 Le Benevole. - 6.4 L'archivio. - 6.5 II romanzesco. - 6.6 II kitsch. 6.7 Collasso: la narrazione impossibile.

\subsection{Auschwitz: l'aporia dell'indicibile}

È sterminato il numero delle narrazioni che ruotano intorno alla Shoah, ma nonostante questo l'evento della tragedia in sé ha continuato a lungo a non essere colto, a restare sfuggente. Le affermazioni di Claude Lanzmann, per esempio, o le radicali prese di posizione di Elie Wiesel, hanno contribuito a gettare un'ombra sulla possibilità di raccontare la tragedia ebraica. La Shoah era indicibile: pretendere di raccontarne l'orrore sarebbe stata la più grave delle trasgressioni (Boswell 2012, 7). Parlando della realizzazione di Shoah (1985), ad esempio, il regista aveva sostenuto una

absolute obscenity in the very project of understanding. Not to understand was my iron law during all the eleven years of the production of Shoah. I had clung to this refusal of understanding as the only possible ethical and at the same time the only possible operative attitude. (Lanzmann 1995, 204)

Mentre Wiesel aveva lanciato una sorta di anatema:

The Holocaust? The ultimate event, the ultimate mystery, never to be comprehended or transmitted. (cit. in Novick 1999, 211) 
Un tabù sulla possibilità di narrare la Shoah, del resto, era già stato posto da Adorno quando nel 1949 aveva dichiarato che «scrivere una poesia dopo Auschwitz è un atto di barbarie» $(1972,22)$. Cristallizzatosi rapidamente in un aforisma lapidario, il celebre monito del filosofo della Scuola di Francoforte esprimeva appieno i dubbi di un'intera epoca rispetto alle possibilità del pensiero di potersi misurare con l'evento insondabile dello sterminio: Auschwitz rappresentava, sul piano della soggettività, una vera e propria cesura epocale.

Tuttavia, con il mutare del panorama storico ancora traumatizzato dagli stermini, il lento affievolirsi delle testimonianze dirette e il passare delle generazioni, chi ha cominciato a raccontare e a rappresentare la Shoah ha cercato di emanciparsi dal veto posto nei confronti della resa finzionale rispetto alla non rappresentabilità dell'evento. Questa emancipazione è passata proprio attraverso il ricorso a quella finzionalità inizialmente rigettata dalle posizioni del filosofo della Scuola di Francoforte.

Rappresentano un esempio in questo senso le dichiarazioni di Giorgio Agamben, che in ambito filosofico aveva sollevato le sue perplessità proprio riguardo al conferire allo sterminio il «prestigio della mistica» $(1998,30)$, ricordando il teologo Giovanni Crisostomo il quale affermava l'assoluta incomprensibilità di Dio sapendo che proprio questo era il modo migliore per glorificarlo:

Dire che Auschwitz è 'indicibile' o 'incomprensibile' equivale a eunphēmein, ad adorarlo in silenzio, come si fa con un dio; significa, cioè, quali che siano le intenzioni di ciascuno, contribuire alla sua gloria. Noi, invece, 'non ci vergogniamo di tenere fisso lo sguardo nell'inenarrabile'. Anche a costo di scoprire che ciò che il male sa di sé, lo troviamo facilmente anche in noi. (30)

Si è cominciato quindi a problematizzare la questione della Shoah affrontando di petto il punto cruciale della sua rappresentazione, e il dogma dell'indicibilità ha cominciato ad essere messo in discussione tramite l'uso di narrazioni sempre più orientate verso la messa in scena finzionale dell'evento. Antitetico rispetto al film di Lanzmann, per esempio, è Shindler's List (1993) di Steven Spielberg, il film che ha provato a far confluire le testimonianze dei superstiti nel racconto della Storia. Un film in cui la Shoah comincia a non essere più un ente metafisico insondabile, quanto piuttosto un evento riscontrabile nella vita degli individui (Bidussa 2009, 106) - nonostante poi l'incontro con Auschwitz resti sfumato e vago e il regista scelga quindi di sorvolarlo.

Per le generazioni successive a quella che ha vissuto i campi di concentramento, quindi, rimane urgente la necessità di ricostruire e raccontare il trauma, ma rispetto all'impasse del secondo dopoguerra comincia a prendere piede una messa in scena dell'evento attraverso storie di finzione. 


\subsection{La figura del testimone e l'età dell'inesperienza}

Mentre si sviluppava il dibattito sulla legittimità della rappresentazione estetica della Shoah, la memoria contemporanea ha cominciato a fare i conti con la lenta scomparsa delle voci dei testimoni, attorno ai quali si è creata un'ambiguità problematica. Con un rovesciamento semantico, infatti, il testimone oltre ad essere osservatore dell'evento traumatico è diventato anche lo spettatore - davanti allo schermo - della narrazione delle vicende altrui.

A partire dalla fine della Seconda guerra mondiale si è inaugurata quella che Annette Wieviorka (1999) ha definito l'«era del testimone»: la figura del testimone affiora dal periodo della Shoah e assume un ruolo significativo nel contesto sociale della cultura contemporanea a partire dal processo Eichmann, «una vera e propria svolta rispetto all'emergere della memoria del genocidio» (Wieviorka 1999, 71).

Scrive Wieviorka che con il processo

inizia una nuova epoca: quella in cui la memoria del genocidio diviene l'elemento costitutivo di una determinata identità ebraica e la sua presenza nello spazio pubblico viene rivendicata con forza [...]. Per la prima volta, un processo si pone esplicitamente l'obiettivo di dare una lezione di storia. Per la prima volta, appare il tema della pedagogia e della trasmissione. (71)

Un anno dopo il saggio di Wieviorka anche il massmediologo John Ellis lavora sul concetto di witness, questa volta però da un'altra prospettiva. A partire dagli anni Cinquanta con l'avvento della televisione sono le masse di telespettatori a svolgere la funzione di testimoni - a distanza - nei confronti dell'esistenza altrui, e la presenza domestica della televisione «has made witness into an everyday, intimate and commonplace act» (Ellis 1999, 36).

In Seeing Things: Television in the Age of Uncertainty Ellis scrive:

Witness is a new form of experience. [It] allows us to experience events at a distance, safe but also powerless, able to over-look but under-act. And, further, we are now able to understand how witness brings us into a complicity with those events. $(1999,15)$

Ellis sottolinea la perdita del legame tra il ruolo del testimone e l'esperienza diretta, perché l'atto del testimoniare si è ormai slegato da qualsiasi altro tipo di esperienza personale. Se quindi in origine il testimone «è associato al destino di colui di cui testimonia» (LéonDufour 1978, 520), e con Wieviorka le vittime 'autorevoli' prossime alla scomparsa ma ancora legate all'evento traumatico testimoniavano la propria esperienza, oggi una massa indistinta di individui diventa passivamente partecipe alla sofferenza dell'altro, rimanendo 
però a distanza di sicurezza dagli effetti della sofferenza reale. Quali conseguenze comporta allora questo divario tra il ruolo massificato del testimone a distanza e l'impossibilità di farsi carico del portato traumatico a cui assiste?

Secondo la nota lettura del pensiero postmoderno la realtà è stata assorbita dal virtuale. Il nostro mondo sembra destinato ad un'indistinzione tra gli eventi-oggetti e le loro rappresentazioni, dove queste ultime si sovrappongono ai primi contraffacendone l'autenticità. L'impatto dei vecchi e nuovi media nella vita dell'individuo sembrano scollegarlo dalla sua esperienza diretta nei confronti degli eventi 'storici'. La realtà è scomparsa - non in senso fisico-materialistico, ma semiologico, come parte di un sistema di segni coerenti prodotto dall'uomo - e il reale è stato assorbito dalla sua simulazione prima televisiva e oggi telematica. È la progressiva riduzione dello spazio ontologico che si comprime su una sola dimensione, la «telemorfosi integrale della società» di cui parla Jean Baudrillard $(2014,79)$, in cui realtà e finzione convergono fino a creare un mondo unico e inseparabile e non esistono più «né finzione né realtà» $(1980,56)$, perché «l'iperrealtà le abolisce entrambe» (56).

Antonio Scurati ha riflettuto sul rapporto tra trasmissione e ricezione dell'evento traumatico nel postmoderno, e a proposito dell'epoca dei mass media, rifacendosi alle tesi di Baudrillard, lo scrittore e accademico italiano ha sostenuto che tutti i grandi eventi storici degli ultimi decenni sono stati per noi degli eventi mediatici, dalla guerra del Golfo trasmessa in diretta televisiva nel gennaio del 1991 agli attentati terroristici dell'11 settembre. In Dal tragico all'osceno. Raccontare la morte nel XXI secolo, Scurati scrive:

La Prima guerra del Golfo stabilì il paradigma della odierna prevalenza dell'immaginario imponendo un rapporto di proporzionalità inversa tra spettacolarità e visibilità: a un aumento esponenziale delle immagini di guerra, corrisponde una progressiva diminuzione della capacità del telespettatore di stabilire, attraverso l'esercizio della vista, una presa conoscitiva sulla realtà. $(2012,66)$

In una sorta di esclusione storica, in cui sembra essere negata la conoscenza del mondo sulla base dell'esperienza vissuta, la società dell'informazione attraverso un continuo bombardamento di immagini virtuali non contribuisce a generare esperienza ma finisce con il requisirla, rendendola impossibile da descrivere senza ricorrere ancora ad altre immagini che non hanno niente a che fare con l'esistenza quotidiana (Giglioli 2011, 18). Scurati la definisce «età dell'inesperienza», in cui la realtà non è più vissuta e la conoscenza non è più acquisita attraverso l'esperienza diretta dell'individuo a contatto con la vita reale: 
è la struttura stessa dell'esperienza a essere andata distrutta nelle condizioni di vita della società tardo-moderna. Il mondo oggi non 'si vive', e la sua conoscenza non riposa più sull'esperienza. Al contrario, l'inesperienza è la condizione trascendentale dell'esperienza attuale. L'inesperienza è la nuova forma di indigenza, il nuovo senso di 'nullatenenza assoluta' da cui nascono i romanzi oggi. L'inesperienza, questo è il nostro stato di povertà oggi, da cui siamo chiamati a fare letteratura [...]. Oggi, più viviamo più siamo inesperti della vita. L'inesperienza si accumula innaturalmente come un tempo si cumulava, naturalmente, l'esperienza. (Scurati 2006, 34-5)

Si crea quindi l'«illusione della presenza, la finzione dell'esperienza» (Scurati 2012, 10), che permette al testimone-spettatore di assistere a ogni tipo di esperienza traumatica facendo propria la focalizzazione del testimone ed esponendosi alla violenza del trauma, rimanendo però al sicuro dai suoi effetti. Ne deriva un nuovo testimone abitatore di una comoda prospettiva, affrancato dalla responsabilità per la morte altrui. Paragonando questo tipo di atteggiamento a una perversione, Slavoj Žižek in Enjoy your Symptom! Jacques Lacan in Hollywood and Out ha scritto di un

universe in which, as in cartoons, a human being can survive any catastrophe [...]; in which one is not forced to die [...]. As such, [it] is the universe of pure symbolic order, of the signifier's game running its course, unencumbered by the Real of human finitude. (2001, 262)

Riassumendo, nel tempo della 'postmemoria'1 si verifica un passaggio critico tra il vuoto in espansione dei testimoni diretti della Shoah da un lato e l'assenza sempre più strutturale di esperienza diretta nei confronti degli eventi storici da parte dell'individuo dall'altro. La problematica connaturata a questo fenomeno è che la sovrapposizione del ruolo di testimone alla figura dello spettatore sembrerebbe privarlo di un mandato sociale che questa veste implicherebbe, trasformandolo quindi in un soggetto indifferente, anestetizzato e depotenziato nelle sue responsabilità etiche rispetto alla comprensione della realtà.

Nell'epoca dello «sciopero degli eventi» (Baudrillard 1993) questa condizione di inesperienza si traduce nella ricerca spasmodica di un'esperienza traumatica, intesa come unico principio valido at-

1 Secondo Marianne Hirsch la postmemoria «describes the relationship that the 'generation after' bears to the personal, collective, and cultural trauma of those who came before» $(2012,5)$, dove il rapporto rispetto al trauma è mediato «not by recall but by imaginative investment, projection, and creation» (5). 
torno al quale costruire la propria identità smarrita per l'assenza di un trauma reale. È il «trauma dell'assenza di trauma» $(2011,7)$ di cui parla Daniele Giglioli nel fortunato Senza trauma. Scrittura dell'estremo e narrativa del nuovo millennio:

[1]'identità contemporanea riesce a pensarsi solo tramite il dispositivo dell'identificazione vittimaria. Io sono ciò che ho subito. E se non ho subito nulla sono nulla. Al vissuto, al centro esatto del vissuto, manca qualcosa di decisivo. Qualcosa di intrattabile, irriducibile, impossibile: per questo devo continuamente mendicare immagini e parole da esperienze che non solo non ho vissuto, ma che non potrei mai in coscienza auspicare di vivere davvero. $(2011,10)$

\subsection{Le Benevole}

Nel caso de Le Benevole (2007) di Jonathan Littell, il romanzo premio Goncourt analizzato nel presente contributo, questo processo si condensa in una forma di scrittura estrema, una «tensione verso qualcosa che eccede costitutivamente i limiti della rappresentazione» (Giglioli 2011, 14): perché la rilevanza del romanzo di Littell è data proprio dalle problematiche che le scelte dell'autore mettono in luce rispetto al mandato etico della letteratura e alla memoria degli eventi narrati, e dal modo in cui l'autore decide di confrontarsi con l'indicibilità di Auschwitz, nel tentativo di superare il tabù di Adorno. Littell dimostrerebbe che

di Auschwitz si può fare romanzo, rinunciando a retroversioni temporali, reticenze, mediazioni. Il che implica una nuova assunzione di responsabilità, che ci riguarda proprio perché l'Olocausto non è distopia e non scardina le leggi del tempo. Non è fuori ma dentro la Storia. (Scaffai 2013, 221)

Ne Le Benevole si concentrano tutte le problematiche trattate fino a questo punto, nel momento in cui Littell sembra voler lacerare la superficie della realtà ${ }^{2}$ per andare alla ricerca dell'origine del trauma, affrontando il potente dramma del genocidio del popolo ebraico per smuovere il lettore-testimone dal suo pericoloso stato di indifferenza. Le domande che pone il romanzo sono allora di portata epocale: è eticamente possibile oggi una narrativa intesa come forma di rappresentazione estetica dell'inimmaginabile? Può la letteratura nel tempo della postmemoria e della società caratterizzata dal ruolo di

2 La copertina dell'edizione italiana Einaudi de Le Benevole raffigura l'opera Concetto spaziale, Attese di Lucio Fontana (1965). 
testimone a distanza farsi carico della trasmissione della memoria della Shoah? Con quali mezzi, e con quali effetti?

Littell, dal canto suo, non manca di porsi in maniera provocatoria nei confronti del lettore, in particolare per quanto riguarda la scelta stessa del narratore protagonista, l'io del romanzo: l'SS Maximilen Aue. Il romanzo narra la storia di questo spregiudicato ufficiale nazista, di cui il lettore segue le vicende attraversando i luoghi chiave dei crimini nazisti: il viaggio dell'orrore di Aue inizia sul fronte orientale nel 1941, dove al seguito dei reparti speciali delle Einsatzgruppen partecipa allo sterminio di Babij Jar in Ucraina, e prosegue poi a Stalingrado dove giunge alla vigilia della disfatta della VI Armata di von Paulus. Ferito gravemente viene rimpatriato e riceve da Himmler il compito di elaborare un sistema logistico per aumentare la produttività dei detenuti nei campi di sterminio. Aue visita quindi Auschwitz e infine fugge in maniera rocambolesca dalla Berlino in macerie del maggio 1945. A questo piano storico e collettivo si intreccia poi quello privato, complesso ed emblematico: Aue infatti è un omosessuale non dichiarato, matricida, è sconquassato da disturbi fisici ed è soprattutto attratto morbosamente dalla sorella gemella Una, con cui ha avuto dei rapporti incestuosi, tramite la quale cerca di ricostruire la propria identità schizofrenica.

Sono numerose e discordanti le interpretazioni che la critica ha provato a fornire rispetto al complesso e stratificato significato del romanzo. Con questo intervento intendo piuttosto circoscrivere il campo di indagine e focalizzare l'analisi su due aspetti specifici de Le Benevole, nel tentativo di dimostrarne il valore emblematico di opera rappresentativa al tempo della postmemoria: l'uso dell'archivio come fonte storicamente attendibile e la strabordante potenza della finzione romanzesca. La particolare sovrapposizione tra questi due registri si risolverà al termine delle oltre 900 pagine del romanzo nell'impossibilità diegetica di mantenere la narrazione da parte dell'io narrante, riuscendo però a tracciare dal punto di vista metodologico un nuovo percorso nell'ambito della trasmissione della memoria della Shoah al tempo della postmemoria.

\subsection{L'archivio}

Nel tempo della postmemoria sia chi scrive sia chi legge non ha vissuto il trauma della Shoah. Le Benevole in questo senso rappresenta l'espressione del romanzo della 'Dopostoria', perché attinge la materia narrativa di cui è composto direttamente dalla tragedia del Novecento, pur essendo scritto da chi appartiene a una generazione di non testimoni. Una generazione - come abbiamo visto in preceden$\mathrm{za}$ - che può impossessarsi di un significato a posteriori e ridare senso e forma alla propria esperienza impoverita 
solo attraverso lo splendore straniato di un'assenza - il trauma che non subiamo, le vittime che non siamo. (Giglioli 2011, 10)

E il caso di Littell è emblematico: nato nel 1967, di famiglia ebraica emigrata negli Stati Uniti a fine Ottocento, lo scrittore consulta uno sterminato archivio documentale attingendo a un tipo di esperienza depositata per supplire alla mancanza di esperienza diretta. Se scrivere di un evento traumatico non è possibile, perché ne manca l'esperienza, allora, come scrive Giglioli ne «Il buco e l'evento»:

È il 'fuori', è l'informazione, è il sapere, non più il vissuto, che si tenta di trasformare in flusso di coscienza. L'immaginazione arriva dall'esterno. La memoria si appropria della storia, la parassita [...]. Tra la scrittura e la realtà si interpone come un corpo luteo, - insieme garante e schermo, filtro e supporto - la materialità del documento, scritto o visivo, memoriale o ufficiale, diretto o indiretto. Da profonda e produttiva, l'immaginazione si fa riproduttiva e intermediale. (2012, 283-4)

Come nota Scurati, il risultato del lavoro di Littell è perciò un romanzo colto e iperdocumentato, in cui le tracce degli studi fatti dall'autore abbondano nella costruzione del testo, ricco di una miriade di riferimenti impliciti ed espliciti a fonti storiche e letterarie. Queste ultime in particolare, come ha notato Julia Kristeva, vanno

de Plutarque et Tertullien à Bossuet, sans oublier Lermontov, le voyageur arabe Ibn Battuta, Dumézil sur les ossètes, Hérodote, Platon évidemment, Joseph de Maistre, Stendhal, Blanchot, le Flaubert de L'Education sentimentale, sans parler de Rebatet, Brasillach, Maurras, Céline, Ambroise de Milan et Augustin découvrant la lecture mentale, Kant, Nietzsche, mais aussi, en mélomane exquis, Mozart, Bach, Brahms, le baroque, notamment français, Schönberg bien sûr, en passant par Watteau et Robespierre. $(2007,27)$

L'uso delle fonti storiche invece si rivela indispensabile per Littell tanto per ricostruire i contesti storici in cui si muove il personaggio Aue quanto, in particolare, per la notevole descrizione del complesso apparato burocratico nazista di cui è parte. Sono soprattutto le ricerche di Raul Hilberg e Christopher Browning a venire apertamente utilizzate - e distorte - dal personaggio stesso, che impegnato a sminuire la portata della Shoah la riduce a questione numerica diluendola nel quadro generale delle vittime di guerra. Le Benevole si apre con una dettagliatissima conta dei morti:

Chi di voi ha mai tentato di contare tutte le persone che conosce o ha conosciuto nella vita, e di paragonare quella cifra irrisoria ai 
numeri che sente alla televisione, quei famosi sei milioni o venti milioni. Facciamo un po' di conti. I conti sono utili, aprono prospettive, rinfrancano la mente. Può essere un esercizio molto istruttivo. (Littell 2007, 15)

I calcoli di Aue vengono compiuti alla luce delle ricerche del monumentale The Destruction of the European Jews di Hilberg:

Lo stimatissimo professor Hilberg, specialista della questione e poco sospettabile di partigianeria, almeno a favore dei Tedeschi, giunge, alla fine di una dimostrazione serrata di diciannove pagine, alla cifra di 5100 000, il che corrisponde grosso modo all'opinione del defunto Obersturmbannführer Eichmann. Vada dunque per la cifra del professor Hillberg, il che, per ricapitolare, fa:

Morti sovietici 20 milioni

Morti tedeschi 3 milioni

Totale parziale

(guerra all'Est) 23 milioni

Endlösung $\quad 5,1 \mathrm{milioni}$

Totale 26,6 milioni, contando che 1,5 milioni di ebrei sono già stati calcolati fra i morti sovietici («Cittadini sovietici uccisi dall'invasore tedesco-fascista», come indica assai discretamente lo straordinario monumento di Kiev). (16)

Le Benevole quindi non è un romanzo storico nel senso stretto del termine: come abbiamo visto, Aue si appropria degli studi storici sulla Shoah per distorcerli e supportare la sua logica negazionista e inserirli nella sua psicopatologia (Kristeva 2007, 26):

La distinzione del tutto arbitraria stabilita dopo la guerra fra le «operazioni militari» da una parte, equivalenti a quelle di qualunque altro conflitto, e le «atrocità» [naziste] dall'altra, perpetrate da una minoranza di sadici e di pazzi, è, come spero di dimostrare, un fantasma consolatorio dei vincitori [...]. La guerra totale è anche questo: il civile non esiste più, e tra il bambino ebreo gasato o fucilato e il bambino tedesco morto sotto le bombe incendiarie c'è soltanto una differenza di strumenti; [...] in entrambi i casi l'uomo o gli uomini che li hanno uccisi credevano che fosse giusto e necessario; se hanno avuto torto, a chi dare la colpa? (Littell 2007, 19)

Aue sostiene inoltre a più riprese che lo sterminio degli ebrei non rappresenti un evento di rottura epistemologica con la Storia, interpretandolo piuttosto come il «formidabile contraccolpo» (Césaire [1999] $2010,48)$ delle pratiche coloniali europee. Questa interpretazione dell'evento, su cui per prima si era soffermata Hannah Arendt con Le origini del totalitarismo (1967) e che mette chiaramente in luce le 
problematiche e i rischi di manipolazione che una pura ricostruzione storica comporterebbe, rappresenta il tentativo da parte dell'autore di ricostruire attraverso l'io narrante le letture che sono state date della Shoah all'interno degli Holocaust Studies. In particolare, Littell si ricollega agli studi che hanno rivisto la Shoah alla luce della decolonizzazione - I dannati della terra di Frantz Fanon (1962), Discorso sul colonialismo di Aimé Césaire (1999) tra gli altri - riecheggiando in particolare quella che Michael Rothberg ha chiamato «the colonial turn in Holocaust studies» $(2009,31)$.

La continuità tra l'amministrazione coloniale europea e il totalitarismo nazista è esemplificata nel romanzo in particolare attraverso due personaggi: il primo è quello del Generalgouverneur Hans Frank, che espone a Himmler il suo progetto architettonico in onore di Hitler:

È un Menschengarten, per dirla alla viennese, un giardino antropologico che desidero creare qui a Cracovia [...]. Fece un ampio gesto al di sopra del plastico. «Si ricorda, mio caro Reichsführer, durante la nostra giovinezza, prima della guerra, quelle Völkerschauen di Hagenbeck? Con famiglie di Samoani, di Lapponi, di Sudanesi? Ne era passata una a Monaco, mio padre mi ci aveva portato, deve averla vista anche lei. E poi ce n'erano ad Amburgo, a Francoforte, a Basilea, avevano un grande successo». Il Reichsführer si strofinava il mento: «Sì, sì, ricordo. Erano esposizioni itineranti, vero?» «Sì. Questa invece sarà permanente, come uno zoo. E non sarà un divertimento pubblico, mio caro Reichsführer, ma uno strumento pedagogico e scientifico. Riuniremo esemplari di tutti i popoli scomparsi o in via di estinzione in Europa, per preservarne così una traccia vivente. Gli scolari tedeschi verranno qui in autobus a istruirsi!» (Littell 2007, 661)

Riallacciandosi al colonialismo francese Frank spiega a Himmler il progetto di finanziare lo zoo su base commerciale come già era accaduto in Francia a fine Ottocento:

«[...] È una bellissima idea. E come pensa di finanziare questo... Völkerschauplatz?» «Su base commerciale. Soltanto l'istituto di ricerche riceverà dei sussidi. Per il giardino, creeremo una AG per raccogliere capitali mediante sottoscrizione. Una volta ammortizzato l'investimento iniziale, le entrate copriranno i costi di mantenimento. Mi sono documentato sulle esposizioni di Hagenbeck; davano notevoli profitti. Il Giardino di acclimatazione, a Parigi, era in perdita costante finché il direttore, nel 1877, non ha organizzato le esposizioni etnologiche di Nubiani e di Esquimesi. Il primo anno hanno avuto un milione di visitatori paganti. Ha continuato fino alla Grande guerra». Il Reichsführer annuiva: «Bella idea». (662) 
Il secondo personaggio è quello di Herr Leland, direttore dell'azienda chimica IG Farben, ex amministratore coloniale dell'odierna Namibia, che ricordando la sua esperienza in Africa riconduce al generale Lothar Von Trotha le pratiche di sfruttamento coloniale del britannico Cecil Rhodes:

Herr Leland si mostrava molto loquace: bevendo un bicchiere dopo l'altro, parlava del proprio passato di amministratore coloniale in Africa sudoccidentale. Aveva conosciuto Rhodes, per il quale nutriva una sconfinata ammirazione [...]. «Una volta Rhodes ha detto: Il colonizzatore non può fare niente di male, ciò che fa diventa giusto. É suo dovere fare ciò che vuole. È stato questo principio, applicato alla lettera, a procurare all'Europa le sue colonie, il dominio sui popoli inferiori [...]. Nel 1907 ho lavorato con il generale von Trotha. Gli Herero e i Nama si erano ribellati, ma von Trotha era un uomo che aveva capito l'idea di Rhodes in tutta la sua forza. Lo dichiarava apertamente: Anniento le tribù ribelli in fiumi di sangue e fiumi di denaro. Solo dopo questo repulisti potrà venir fuori qualcosa di nuovo. Ma già a quell'epoca la Germania si stava indebolendo, e von Trotha è stato richiamato [...]». (684)

È proprio sull'inaffidabilità storica della narrazione - la quale come abbiamo visto si appoggia pure ad un gran numero di studi e ricerche - che la maggior parte della critica si è scagliata. ${ }^{3}$ Ma le critiche rivolte all'uso distorto e disinvolto dei dati e degli studi storici da parte di Littell-Aue non possono essere del tutto prese in considerazione, come si è espressa Kristeva a riguardo:

Je rappellerai seulement le mot de Magritte, sous l'image d'une pipe: «Ceci n'est pas une pipe». Les Bienveillantes n'est pas un ouvrage d'historien, pas plus qu'une analyse de la Shoah: c'est une fiction qui restitue l'univers d'un criminel. Ne lisons donc pas le roman avec les critères que requiert une œuvre d'historien, mais avec ceux que requiert une œuvre d'imagination. (2007, 27)

La questione è fondamentale e troppo spesso posta in secondo piano, perché è solamente provando a considerare Le Benevole come un'opera romanzesca che si può comprendere la portata della riflessione sollevata da Littell sulla trasmissione della memoria:

C'est sur ce plan, et sur ce seul plan que des questions surgissent, autrement plus douloureuses, voire insolubles, que les objections des historiens et des moralistes. (Kristeva 2007, 27)

3 Si veda a proposito lo studio di Paul-Eric Blanrue, Les Malveillantes, enquête sur le cas Jonathan Littell (2006). 


\subsection{Il romanzesco}

L'uso spinto del romanzesco rappresenta l'altra faccia della ricerca del trauma da parte di Littell, che liberato anagraficamente dal peso della responsabilità diretta nei confronti della Shoah può provare a muoversi liberamente alla ricerca del suo significato, attraverso le possibilità del romance. Ė infatti la struttura dell'opera di finzione romanzesca a modellare Le Benevole: l'intera biblioteca di Littell, tutte le fonti e gli studi preparatori vengono contaminati dal potente flusso narrativo che ricolloca l'archivio all'interno della finzione del romanzo (Scurati).

Northrop Frye ha collocato il romance a metà strada tra la forma del novel e quella del mito: come scriveva in Anatomia della critica (1969), la tendenza del romance è quella di

suggerire schemi mitici impliciti in un mondo più strettamente associato all'esperienza umana. $(1969,182)$

A partire dal titolo, il romanzo di Littell si richiama direttamente alle figure mitologiche delle Eumenidi ('benevole') eschilee, responsabili, in particolare, della persecuzione dei colpevoli di matricidio. È poi lo schema dell'intera trilogia dell'Orestea a strutturare il romanzo di Littell: ${ }^{4}$ così come nell'Agamennone Clitennestra uccide il marito assieme ad Egisto, così Aue è convinto che la madre, «odiosa cagna» (Littell 2007, 497), sia responsabile assieme al patrigno Moreau della scomparsa del padre:

«Non è morto», mormorai appassionatamente [...]. «È come se lo avessero assassinato. Assassinato a colpi di documenti. Che vergogna! Per i loro desideri colpevoli» (510)

Come Oreste vendica la morte del padre nelle Coefore, così Aue si macchia di matricidio e dell'omicidio di Moreau e per questo, come nelle Eumenidi, viene perseguitato lungo tutto l'arco della narrazione dai due detective Weser e Clemens. Le indagini dei due personaggi improbabili, descritti come «due macchiette» (712) somiglianti «a una coppia di sbirri da film americano» (711), virano però presto nei cliché del poliziesco di consumo tra interrogatori stereotipati, appostamenti e inseguimenti inverosimili tra Berlino, Budapest e la Pomerania. Aue è portato all'esasperazione, mentre la narrazione si fa sempre più grottesca e surreale:

4 Per una lettura intertestuale de Le Benevole con le tragedie di Eschilo si veda Mercier-Leca 2007. 
«Ma cosa volete, in fin dei conti?» urlai ancora una volta. «Te l'abbiamo detto, - mormorò Clemens: - vogliamo giustizia». «La città è in fiamme! - esclamai. - Non ci sono più tribunali! Tutti i giudici sono morti o se ne sono andati. Come volete farmi giudicare?» «Ti abbiamo già giudicato, - disse Weser [...]. - Ti abbiamo giudicato colpevole». (937)

Sempre nella sua analisi sul romance, Frye individuava alcune fasi che ne determinano la peculiare «forma a sviluppo progressivo» $(1969,247)$ : una di queste riguarda «un mondo arcadico e pastorale» (265) che corrisponde all'«innocenza dell'eroe» (265). Ne Le Benevole il pensiero di Aue è sempre rivolto al passato, verso quella che lui stesso definisce come «l'età della pura innocenza, sontuosa, magnifica» (Littell 2007, 393):

Là i nostri giochi dilagavano nei campi, nei boschi di pini neri e nella vicina macchia, che vibrava del frinire delle cicale e del ronzio delle api nella lavanda, il cui odore veniva a coprire gli aromi del rosmarino, del timo e della resina, mescolati, verso la fine dell'estate, anche con quello dei fichi che divoravamo fino alla nausea, e poi, più lontano, il mare e gli scogli caotici che formavano quella costa frastagliata, fino a un isolotto in pendio che raggiungevamo a nuoto o in canotto. (392)

Tuttavia, come notava sempre Frye, il mondo arcadico nel romance è caratterizzato da elementi che hanno a che fare con «l'aspetto femminile [...] delle immagini sessuali» $(1969,265)$, dove il tipo di relazione prevalente è «l'amore del fratello per la sorella» (265), esattamente quello che ha condizionato, devastandolo, lo sviluppo psichico di Aue, impedendogli di sviluppare una propria identità sessuale. Aue infatti è innamorato della sorella Una con la quale ha un rapporto simbiotico e incestuoso:

Ci mettevamo a prua, insieme, e guardavamo le onde incresparsi, poi ci guardavamo l'un l'altra, e tramite quello sguardo, attraverso l'amarezza della nostra infanzia e il rombo sovrano del mare, accadde qualcosa, qualcosa di irrimediabile: l'amore, dolce-amaro, fino alla morte. Ma allora era ancora solo uno sguardo. Non rimase tale a lungo. Non capitò subito, forse un anno dopo; quando scoprimmo quelle cose, un piacere senza limiti riempì la nostra infanzia. (Littell 2007, 196)

Un periodo che viene ricordato allora «come un'epoca felice o età dell'oro perduta» (Frye 1969, 265), dove però, allo stesso tempo, aleggia irrimediabile la presenza di un «tabù morale» (265), che nel caso di Aue corrisponde a quello dell'incesto: «Ci trovavamo», ricorda 
Aue, «fra l'Età dell'Oro e la Caduta» (Littell 2007, 464). Il romance infatti, «tradotto in termini onirici» (Frye 1969, 257), è la ricerca dell'appagamento della libido «che liberi l'io stesso dalle ansie della realtà» (257): perciò coloro che ostacolano l'impresa del personaggio sono «figure sinistre [...] che hanno chiaramente un'origine paterna o materna» (257). Ne Le Benevole sono la madre e il patrigno Moreau a separare traumaticamente Aue dalla sorella Una:

E poi un giorno [...] ci sorpresero. Furono scenate infinite, mia madre mi dava del maiale e del degenerato. Moreau piangeva, e fu la fine di tutto ciò che è bello [...], e così [...] cominciò un incubo durato molti anni e che, in un certo senso, continua ancora. (Littell 2007, 196)

In questi termini il matricidio di Aue/Oreste può fungere da molla per la liberazione (fallita) dell'io, che in tal modo potrebbe perpetuare il proposito del godimento libidico, recuperando il tempo dell'innocenza e ricostruendo la propria identità: il matricidio, come scrive Kristeva, è una necessità vitale dell'uomo, «conditio sine qua non della nostra individuazione» $(1988,31)$.

Il romance perciò si innesta su una vicenda raccontata in maniera apparentemente incontestabile e ne scompagina la forma, esasperandone la portata di significato, mirando volontariamente a disturbare la comoda posizione del destinatario. Così è alla luce della riappropriazione della libertà di finzione dell'autore che si legge la scandalosa adozione del punto di vista dell'SS Aue, che fin dall'inizio del romanzo può rivolgersi ai lettori in nome di una presunta fratellanza condivisa:

Fratelli umani, lasciate che vi racconti com'è andata. Non siamo tuoi fratelli, ribatterete voi, e non vogliamo saperlo. Ed è ben vero che si tratta di una storia cupa, ma anche edificante, un vero racconto morale, ve l'assicuro. Rischia di essere un po' lungo, in fondo sono successe tante cose, ma se per caso non andate troppo di fretta, con un po' di fortuna troverete il tempo. E poi vi riguarda: vedrete che vi riguarda. (Littell 2007, 5)

Il risultato che ne consegue si può facilmente misurare nella disturbante ambiguità in cui si trova costretto il lettore, combattuto tra la repulsione e l'empatia:

Littell ably toes the line between alienation and empathy. Max Aue belongs, on the one hand, to the elite of the SS, but he is, on the other hand, close enough to us to allow us a certain identification. He is surrounded by SS men, who are either too narrow-minded or fanatical for us to identify with. Arguments between these SS 
men and Aue are not infrequent, and it is here that we inevitably find ourselves on Aue's side. (Theweleit, Nunan 2009, 28)

Se questa focalizzazione può essere considerata come la conseguenza diretta, sul piano narrativo, dell'emancipazione della soggettività nell'età dell'inesperienza, lo diventa allora anche l'indifferenziazione tra le vittime e gli aguzzini: la provocazione del romanzo infatti, ancora più che destabilizzare il lettore che si trova a leggere una storia narrata in prima persona da un carnefice, risiede nel porre da un lato la coppia vittima-carnefice, dall'altro il lettore-spettatore (Scurati 2012, 13-14). L'appiattimento della figura dell'oppressore su quella della vittima e la ricollocazione del ruolo di entrambe le parti in causa in una sola figura relativizzata, appartenente a un tempo passato non più esperibile, rappresenta la riproposizione in chiave letteraria dell'attuale svuotamento dello sguardo e della mancanza della responsabilità per il trauma altrui, che in questo modo finisce con l'essere solo 'guardato'. Proprio per questo motivo Scurati ha scritto che Le Benevole rappresenta una delle opere letterarie più importanti e pericolose del nostro tempo, oggi che il concetto di 'testimone' può essere inteso sia come sopravvissuto alla Shoah, sia come spettatore televisivo:

Il vortice di vischiosa complicità in cui Littell ci trascina non si sprigiona dal risucchio nel mondo interiore del fittizio nazista Max Aue, ma dalla struttura voyeuristica che ci attira verso la posizione, tutta esteriore, dello spettatore. Rischiamo cioè di condividere con il nazista la sua esteriorità rispetto alla vittima. È il rischio insito in ogni spettacolo della violenza: la nazificazione dello sguardo. (Scurati 2014, 91)

Molto spesso nel romanzo ebrei e nazisti vengono infatti equiparati, e il lettore finisce così con il ritrovarsi esposto a un flusso continuo di immagini che azzera ogni differenza e identità. Se infatti

Gli ebrei sono i primi nazionalsocialisti, da quasi tremilacinquecento anni ormai, da quando Mosè ha dato loro una Legge per separarli per sempre dagli altri popoli (Littell 2007, 440)

e «tutti erano intercambiabili, le vittime come i carnefici» (100), i nazisti, secondo questa prospettiva, hanno voluto sterminare il popolo ebraico per auto-annientarsi:

Uccidendo gli ebrei, - diceva, - abbiamo voluto uccidere noi stessi, uccidere l'ebreo che è in noi, uccidere quello che in noi somigliava all'idea che ci facciamo dell'ebreo. Uccidere in noi il borghese panciuto che conta i suoi soldi, che rincorre gli onori e sogna il potere, ma un potere che si raffigura sotto l'aspetto di un Napole- 
one III o di un banchiere, uccidere la moralità ristretta e rassicurante della borghesia, uccidere l'economia, uccidere l'obbedienza, uccidere la servitù del Knecht, uccidere tutte quelle belle virtù tedesche. Perché non abbiamo mai capito che quelle qualità che attribuivamo agli ebrei chiamandole bassezza, fiacchezza, avarizia, avidità, sete di dominio e facile cattiveria sono qualità essenzialmente tedesche, e che se gli ebrei danno prova di queste qualità, è perché hanno sognato di somigliare ai Tedeschi, di essere tedeschi, è perché ci imitano servilmente come l'immagine stessa di tutto ciò che è bello e buono in Alta Borghesia, il Vitello d'oro di coloro che rifuggono l'asprezza del deserto e della Legge. O forse perché facevano finta, forse hanno finito per adottare quelle qualità quasi per cortesia, per una forma di simpatia, per non mostrarsi così distanti. E noi, invece, il nostro sogno di Tedeschi era di essere ebrei, puri, indistruttibili, fedeli a una Legge, diversi da tutti e sotto la mano di Dio. (846)

L'esempio più emblematico di questa scandalosa sovrapposizione tra l'uomo tedesco e l'uomo ebreo è rappresentato da Hitler, che nel romanzo personifica l'apice di questo gioco perverso:

Dopo i discorsi introduttivi, apparve il Führer. Sbarrai gli occhi: sulla testa e sulle spalle, sopra la semplice uniforme feldgrau, mi sembrava di vedere il grande scialle a righe bianche e azzurre dei rabbini. [...] Scrutai la copertura di vetro: era possibile che si trattasse di un gioco di luci? Vedevo distintamente il suo berretto; ma sotto credevo di distinguere delle lunghe treccine, che scendevano dalle tempie sui risvolti, e sulla fronte, uno dei filatteri (tefillin), le scatolette di cuoio contenenti i versetti della Torah. Quando alzò il braccio, mi parve di scorgere sulla manica l'altro filatterio di cuoio; e dalla giacca non sbucavano forse le frange bianche di quello che gli ebrei chiamano talit? Non sapevo cosa pensare. Osservai i miei vicini: ascoltavano il discorso con un'attenzione solenne, il funzionario annuiva con aria compresa. Non notavano niente? Ero l'unico a vedere quello spettacolo inaudito? [...] Forse, mi dissi, sconvolto, è la storia del re nudo: tutti vedono come stanno le cose, ma lo dissimulano, contando che il vicino farà lo stesso. (451-2)

Tramite le strategie della finzione narrativa Littell può quindi mettere in scena le vicende del romanzo, i cui processi di traduzione in immagine finiscono però con il degenerare nel corso della narrazione: com'è noto infatti Le Benevole si caratterizza per le interminabili e ripetitive descrizioni delle violenze compiute dai nazisti, dai massacri delle Einsatzgruppen in Ucraina ai campi di concentramento, fino alla deriva allucinata del protagonista, vittima della sua stessa psicopatologia passivo-masochistica. Il tutto è descritto minuziosamente 
e in maniera impietosa da Aue, che si sofferma soprattutto sui dettagli più raccapriccianti e sconvolgenti della brutalità nazista. Si tratta di scene storicamente documentate, perfettamente collocate temporalmente e geograficamente, che lasciate però in mano alla libertà narrativa del personaggio, viziata dal suo sguardo pornografico, virano verso l'inaudito, il raccapricciante, nel kitsch spinto all'estremo.

Vediamo alcuni esempi. Dopo aver usato i camion per gasare uomini, donne e bambini ebrei a Kharkov, la scena che viene descritta da Aue è resa in maniera forzatamente rivoltante, volutamente impietosa:

I corpi erano coperti di merda e di vomito [...]. Anche Findeisen, che guidava il camion, ha respirato del gas e vomitava dappertutto. $(2007,170)$

Il baratro più spaventoso in questo senso si apre davanti agli occhi del lettore di fronte all'immensa fossa ai piedi del burrone di Babij Jar, dove in pochi giorni tra la fine di settembre e l'ottobre del 1941 vennero giustiziati tra i 100.000 e i 150.000 ebrei, rom e prigionieri di guerra. Qui

Un tremendo odore di escrementi sovrastava quello del sangue, al momento di morire molti defecavano. (125)

Aue si avvicina alla fossa:

Per raggiungere alcuni feriti, bisognava camminare sui corpi, si scivolava tremendamente, le carni bianche e molli cedevano sotto gli stivali, le ossa si spezzavano a tradimento e mi facevano inciampare, affondavo fino alle caviglie nella melma e nel sangue. Era orrendo e mi riempiva di un acuto senso di disgusto, come quella sera in Spagna, nella latrina con gli scarafaggi [...] Camminare sui corpi degli ebrei mi dava la stessa sensazione. (126-7)

Ancora, camminando tra i cadaveri, Aue si trova di fronte a una giovane donna agonizzante:

continuavo a spararle e la sua testa si era spaccata come un frutto, allora il mio braccio si staccò da me e se ne andò da solo per il burrone, sparando qua e là, io gli correvo dietro, con l'altro braccio gli facevo segno di aspettarmi, ma lui si rifiutava, rideva di me e sparava sui feriti da solo, senza di me. (127)

Come ha notato Scurati, il giudizio impietoso che Godard applicava a Schindler's List di Spielberg potrebbe valere anche per Le Benevole, quando l'oscenità dello sguardo del personaggio invece di svelare la tragedia della Shoah contribuisce a nasconderla; infatti 
A giudizio del cineasta francese [...] nella realizzazione di Schindler's List, fabbricando una falsa apparenza della più grande tragedia del Ventesimo secolo attraverso una perfetta messa in scena, e dunque generando una falsa percezione di realtà nel pubblico, un simulacro d'esperienza di ciò che invece sarebbe dovuto rimanere avvolto dal terrore sacro che suscita in noi l'Altro assoluto, l'inimmaginabile e l'irrappresentabile, il cinema avrebbe tradito se stesso lasciando trionfare l'osceno a scapito del tragico, mettendo proprio l'osceno in luogo del tragico. (Scurati 2014, 89)

È il trionfo del kitsch. Ma l'uso che ne fa Littell, lontano dalla gratuità dell'épater le bourgeois, sembra piuttosto corrispondere a delle precise scelte dell'autore.

\subsection{Il kitsch}

Leggendo gli esempi appena riportati tratti dal romanzo si può evidentemente notare come la possibilità di narrare gli eventi concessa ad Aue venga violentemente distorta, finendo presto per degenerare nell'estremizzazione pornografica. Che cosa significa questo sadismo voyeuristico? Come non tacciarlo immediatamente di oscenità? Si tratta, come più volte la critica ha sottolineato, di «added literary value? Or pornographic voyeurism?» (Kuon 2012, 34).

Tra gli antipodi del dettaglio narrativamente funzionale da un lato e il voyeurismo autoreferenziale dall'altro, estremi entro cui oscilla l'interpretazione critica della minuziosa ed insostenibile oscenità narrativa de Le Benevole, esiste forse una terza lettura, connaturata tanto alla natura dell'io narrante quanto alle strutture stesse del romance: il kitsch. Ci sono due motivi che possono spiegare l'uso del kitsch all'interno della narrazione di Aue.

Innanzitutto il kitsch - come sottolineato nel capitolo «Romance e Shoah» di questo volume a proposito di Marco, il protagonista de L'impostore di Javier Cercas - è strettamente collegato al romanzesco, e rappresenta in un certo senso la sua estremizzazione: il kitsch può essere cioè la degenerazione del romanzesco. La problematicità legata a questo aspetto è piuttosto importante: perché se è vero che il romanzesco è connaturato alle storie che ruotano intorno alla Shoah, allora anche il kitsch deve esserlo per forza.

Secondo Hermann Broch, il cui nome è strettamente legato al concetto di kitsch (Broch 1990), questo sarebbe responsabile soprattutto di sostituire alla categoria etica quella estetica. Questo aspetto potrebbe rappresentare un tratto - certo non quello centrale, ma comunque fondamentale - della estrema libertà connaturata alla rappresentazione romanzesca de Le Benevole, del tutto affrancata nel portare avanti le sue istanze rispetto a basi etiche o morali che in altri ambiti 
circoscriverebbero invece la possibilità di indagine intorno all'oggetto. Infatti, come notato da Peter Kuon in Writing the Holocaust Today. Critical Perspectives on Jonathan Littell's The Kindly Ones,

Between two poles - a commitment to historical precision and the desire for artistic creation - the author opts for aesthetics over documentation. Ethics then falls by the wayside. $(2012,34)$

Il secondo motivo che potrebbe giustificare la degenerazione nel kitsch è dovuto alla natura fascista che caratterizza Aue, nel momento in cui, come vedremo, è proprio kitsch a prestarsi perfettamente come supporto per la falsa moralità del narratore:

kitsch as an aesthetic category and falsehood as a moral one are intertwined. (Kuon 2012, 39)

Soffermiamoci su questo aspetto. Ne Il secco e l'umido (2009) Littell dimostra chiaramente quanto il kitsch sia connaturato alla natura del fascista e alla sua narrazione: in questo saggio, al quale ha lavorato durante stesura de Le Benevole, Littell analizza infatti la struttura mentale del fascista belga Léon Degrelle, fondatore del Parti Rexiste e della legione Wallonie, a partire dal romanzo La campagne de Russie. Per compiere questa operazione Littell si serve delle teorie psicanalitiche che Klaus Theweleit ha utilizzato in Männerphantasien per definire la struttura mentale dell'uomo fascista: secondo il sociologo l'uomo fascista, definito come «maschio-soldato non completamente nato» (Littell 2009, 114) perché non completamente separato a livello inconscio dalla figura materna, è dotato di una corazza a difesa del proprio Io. Si tratta di

un Io esteriorizzato che si presenta come una "corazza" [...]. Tale armatura trattiene nell'interiorità, a cui il fascista non ha accesso, tutte le sue pulsioni, le sue funzioni desideranti assolutamente informi perché incapaci di oggettivazioni. Ma questo Io-corazza non è mai perfettamente ermetico, anzi è fragile [...]. Nei momenti di crisi si frantuma, e il fascista rischia di essere travolto dalle sue stesse produzioni desideranti incontrollabili, dalla "dissoluzione dei limiti personali". Per sopravvivere, esteriorizza ciò che lo minaccia dall'interno, e allora tutti i pericoli assumono per lui due forme, intimamente connesse: quella del femminile e quella della liquidità. (Littell 2009, 20)

Strumento indispensabile per interpretare la figura oresteica, femminilizzata e matricida di Aue, e per leggere Le Benevole alla luce delle devastanti «déflagrations pulsionnelles de l'homosexualité passive» (Kristeva 2007, 27) del protagonista, l'analisi serrata condotta ne Il 
secco e l'umido si dimostra altrettanto rilevante, nel nostro caso, soprattutto per interpretare il kitsch che contraddistingue la narrazione dell' 'uomo fascista' Aue. Per Littell stesso, infatti, un piano di analisi necessario per comprendere la narrazione di Degrelle è prima di tutto proprio quello del kitsch, costitutivo dell'uomo fascista e soprattutto della sua narrazione, filtro attraverso il quale l'io narrante proietta la propria peculiare struttura inconscia. Perché «quando il fascista scrive» $(2009,25)$, rileva Littell, l’oggetto della sua attenzione «assum[e] un significato completamente diverso da quello di una realtà osservata oggettivamente» (25): il testo del fascista «opera [...] ciò che Theweleit chiama la "conservazione dell'Io"» (27), la quale passa attraverso una serie di coppie di opposti dove il secondo termine «rappresenta la minaccia che incombe sull'Io-corazza» (27-8) mentre il primo quelle qualità che al contrario permettono al fascista di rafforzarlo evitando la dissoluzione psichica, «pericolo ben più grave della disfatta militare» (28). Littell individua numerose coppie di opposti che utilizzerà poi per analizzare gli scritti di Degrelle: la principale è quella tra secco e umido;

ci sono inoltre lo strutturato e l'informe, il duro e il molle, l'immobile e il brulicante, il rigido e il flaccido, l'eretto e lo sdraiato, il pulito e lo sporco, il cotto e il crudo, il sazio e l'affamato, il glabro e il peloso, il limpido e il torbido, il trasparente e l'opaco, l'appannato e il lucente, il morbido e il vischioso, e così via. (28)

Ritornando a Le Benevole allora, se da un lato la libertà narrativa concessa dal romance racchiude in sé la possibilità della sua stessa estremizzazione in kitsch, perché sganciata per sua natura dai vincoli etici, dall'altro è il kitsch stesso a rappresentare una forma costitutiva dell'autonarrazione nazista, in quanto parte integrante e proiezione del suo Io instabile.

Proviamo allora a considerare valide queste due motivazioni alla base dell'uso del kitsch nel romanzo di Littell, escludendo quindi la gratuità del suo uso fine a se stesso. Quali potrebbero essere le conseguenze di questo uso in termini di attendibilità narrativa? Le conseguenze di questo approccio sembrano avere delle ricadute proprio sulla delicata trasmissione della memoria nell'età dell'inesperienza: assecondando questa prospettiva il problema si sposta allora dal piano della narrazione a quello occupato dal lettore, investendo, in senso lato, la sua realtà.

In Apocalittici e integrati, Umberto Eco scriveva:

Articolandosi così come una comunicazione artistica in cui il progetto fondamentale non è quello di coinvolgere il lettore in una avventura di scoperta attiva, ma semplicemente di piegarlo con forza od avvertire un determinato effetto - credendo che in questa 
emozione consista la fruizione estetica - il Kitsch apparirebbe come una forma di menzogna artistica. $(1964,69)$

L'uso del kitsch produce innanzitutto una narrazione falsa. È quanto nota anche Cercas ne L'impostore riferendosi a una «menzogna narcisistica che nasconde la verità dell'orrore e della morte» (2015, 179), che esasperando il gesto di mostrare in realtà nasconde, uno strumento di cui il narratore si serve

per il suo assiduo esercizio di occultamento della realtà, per non conoscerla o riconoscerla, per non conoscere sé stesso, o per non riconoscersi, (179)

una forma di dismisura, di falsa organicità contestuale - e dunque ancora come menzogna, truffa perpetrata non a livello dei contenuti ma della stessa forma della comunicazione. (Eco 1964, 84)

Lo stesso Eco ha scritto inoltre che il kitsch rappresenta anche ciò che è consumato, che arriva al pubblico perché è consumato,

e che si consuma (e quindi si depaupera) proprio perché l'uso a cui è stato sottoposto da un gran numero di consumatori ne ha affrettato e approfondito l'usura. $(1964,100)$

Un aspetto che ha a che fare con la ricezione del messaggio da parte del lettore, con l'assuefazione contemporanea alla violenza tradotta in immagini, diffusa e pervasiva, a cui assiste il testimone dell'età dell'inesperienza. Il problema quindi è connaturato anche alla ricezione del messaggio: facendo nostra una citazione di Eco, la narrazione di Aue infatti non sarebbe kitsch solamente perché

stimola effetti sentimentali, ma perché tende continuamente a suggerire l'idea che, godendo di questi effetti, il lettore stia perfezionando una esperienza estetica privilegiata. E quindi, a caratterizzarlo come brano Kitsch, non intervengono solo i fattori linguistici interni al messaggio, ma anche l'intenzione con cui l'autore lo "vende" al pubblico, nonché l'intenzione con la quale il pubblico vi si rivolge. In questo caso ha ragione Broch quando ricorda che il Kitsch non riguarda tanto l'arte, quanto un comportamento di vita, poiché il Kitsch non potrebbe prosperare se non ci fosse un Kitsch-Mensch, che ha bisogno di una tale forma di menzogna per riconoscervisi. Allora il consumo del Kitsch apparirebbe in tutta la sua forza negativa, come una continua mistificazione, una fuga dalle responsabilità che l'esperienza dell'arte invece impone. $(1964,71-2)$ 
Abbandonato lo sdegno rispetto all'uso provocatorio delle potenzialità del romanzesco da parte di Littell, è possibile cogliere la vera problematica alla base della narrazione sulla Shoah nel tempo della postmemoria. È proprio la presenza del kitsch a metterla in luce, esasperando l'illusione della presenza da parte del lettore e la finzione dell'esperienza dell'evento a cui assiste:

accomodandosi in poltrona con in grembo le mille pagine del romanzo, il lettore avrà l'impressione di aver assistito allo sterminio di milioni di persone; ne ricaverà non solo e non tanto la conoscenza intellettuale di che cosa sia stata la Shoah [...] ma, usurpando il posto del testimone, ne ricaverà l'illusione di averla in qualche misura rivissuta nell'immaginazione. La questione posta dal romanzo di Littell, questione vasta quanto la nostra epoca, è dunque quella della possibilità estetica e della liceità etica di una narrativa d'immagine sull'inimmaginabile. (Scurati 2014, 89)

È attraverso il massiccio ricorso al kitsch che il lettore-spettatore prova l'illusione della presenza rispetto agli eventi narrati; tuttavia forzatamente riproposta, violentata e portata all'esasperazione, l'immagine perde consistenza, si svaluta e tradisce il suo scopo testimoniale. Così facendo Littell dimostra quanto possa essere reale il rischio di tradurre il trauma della Shoah attraverso immagini svilite, che

invece di 'strappare', invece di sederci sul bordo di un'esperienza lacerante, le fa[nno] velo, (Scurati 2012, 11)

producendo una «banalizzazione della banalità del male» (11). L'immaginario del romanzo mette a nudo la derealizzazione della realtà: in questo modo per il nuovo testimone, lo spettatore,

nemmeno l'annientamento dell'essere umano significa più qualcosa in se stesso, ma assume il proprio significato per rifrazione dalla diffusione mediatica delle immagini della sua distruzione. (66)

Con le parole di Giglioli, quello de Le Benevole

non è tanto un sapere sullo sterminio quanto un lento apprendistato all'indifferenza, all'assuefazione, alla perdita di valore del mondo e della vita. ${ }^{5}$

5 D. Giglioli, «Dietro il muro della finzione», Il Manifesto, 30 novembre 2007. 


\subsection{Collasso: la narrazione impossibile}

L'uso del romanzesco può rappresentare una potente spinta per la trasmissione della memoria; ma è altrettanto vero che per la sua connaturata mancanza di aderenza alla realtà potrebbe comportare anche un pericolo, e allontanandosi dalla verità storica finirebbe per falsificarla. È quanto notato anche da Liran Razinsky, quando, analizzando la figura di Aue sotto una diversa prospettiva, ha scritto che la narrazione de Le Benevole «contains one kind of writing that documents and another that rebels against documentation» (2010, 185). Pur offrendo al lettore una nuova, eventuale possibilità di 'vedere' il trauma della Shoah attraverso la prospettiva del carnefice, l'«aesthetic of excess» (Sandberg 2014) che configura le modalità narrative del romance rivela quindi l'assuefazione e il distacco che sotterraneamente minacciano la memoria culturale della Shoah nel tempo della postmemoria.

Se Le Benevole, scritto e pubblicato nel tempo della scomparsa degli ultimi testimoni, cerca di ristabilire un contatto con il trauma storico, ricercandone il senso perduto - là dove la Storia non rappresenta ormai più la semplice materia narrativa da cui attingere, ma una presenza incombente a cui guardare nel tentativo di costruire la propria identità 'senza trauma' -, l'uso del romanzesco all'interno di una cornice ricostruita storicamente è destinato a far collassare la narrazione nella convergenza tra i due piani di finzione e affidabilità storica. L'iperdettagliata realtà descritta nel romanzo costruita su un enorme uso di fonti (l'archivio) e al contempo la mancanza di plausibilità del narratore, strutturato sul paradigma del kitsch (il romanzesco), sembrano infatti convergere nell'episodio-chiave del ferimento di Aue a Stalingrado, circostanza che fa conflagrare ricostruzione realistica e degenerazione romanzesca. A rappresentare la collisione sulla quale realtà storica e finzione letteraria si sovrappongono senza coincidere è un «buco», ${ }^{6}$ il foro che un proiettile ha lasciato nella fronte di Aue:

la mia testa era attraversata da un buco, uno stretto corridoio circolare, un pozzo favoloso, chiuso, inaccessibile al pensiero, e se era vero, allora niente era più come prima, come avrebbe potuto esserlo? La mia concezione del mondo adesso doveva riorganizzarsi intorno a quel buco. Ma la sola cosa concreta che potevo dire era: mi sono risvegliato, e niente sarà mai più come prima. (Littell 2007, 422) 
Nel corso del romanzo Aue funziona come un vero e proprio occhio rivolto verso tutto ciò che lo circonda; attratto e al contempo respinto dal trauma in cui è immerso, è ossessionato dallo sguardo:

Volevo chiudere gli occhi, o coprirli con una mano, e al tempo stesso volevo guardare, guardare a sazietà e tentare di comprendere con lo sguardo quella cosa incomprensibile, lì, davanti a me, quel vuoto per il pensiero umano. (35)

Dopo essere stato gravemente ferito a Stalingrado però lo sguardo dell'occhio finisce paradossalmente per imprimersi fisicamente nella sua carne, isolandosi nel suo corpo: il foro nella fronte gli concede cioè una vista 'altra', allucinata e distorta, proiettando «su quel mondo una luce cruda, livida, implacabile» (497):

Che cosa aveva fatto alla mia testa quel proiettile? Mi aveva confuso irrimediabilmente il mondo, o mi aveva davvero aperto un terzo occhio, quello che vede attraverso l'opacità delle cose? (454)

È da questa ferita che scaturisce la sua 'cecità' rispetto al trauma, come si può dedurre risalendo alla Critica dell'occhio (1972) di Georges Bataille a cui Littell si riferisce in maniera piuttosto evidente con l'episodio del ferimento di Aue. Bataille ipotizzava che la ghiandola pineale situata nel cervello fosse in realtà un terzo occhio, fatto per vedere il sole:

io non esitavo a pensare seriamente alla possibilità che quest'occhio straordinario finisse per farsi strada attraverso la parete ossea della testa, perché credevo necessario che dopo un lungo periodo di servilità gli esseri umani avessero un occhio speciale per il sole (mentre i due occhi che sono nelle orbite se ne allontanano con una specie di ostinazione stupida). $(1972,81)$

Questo terzo occhio o 'occhio pineale' veniva associato da Bataille all'ano, due apparati analoghi e capovolti disposti secondo un asse verticale in cui la visione «è lacerata e strappata dagli scoppi del sole che essa fissa» (94):

Mi raffiguravo l'occhio in cima al cranio come un orribile vulcano in eruzione, proprio con il carattere losco e comico che si attribuisce al di dietro e alle sue escrezioni. Ora l'occhio è senza alcun dubbio il simbolo del sole abbagliante, e quello che io immaginavo in cima al mio cranio era necessariamente infuocato, essendo votato alla contemplazione del sole al sommo del suo splendore. (80) 
Secondo Denis Hollier, che al tema dell'occhio in Bataille ha dedicato lo studio Against Architecture: The Writings of Georges Bataille (1992), l'occhio pineale non costituisce però un vero e proprio riferimento al sistema visivo, dato che «this is an eye that is seen, not one that sees: a blind eye» (Hollier 1992, 78). L'occhio pineale è cieco perché non vede,

blind because, from the moment it intervenes, it prevents the (mind's) eye from seeing; blind also because it is not visible. (96)

Per Bataille l'occhio pineale rappresenta lo scacco del pensiero logico, il punto cieco del razionalismo occidentale,

the blind spot of rationalist, utilitarian economy, the whole where the edifice of thought is spent, swallowed up, ruined; where notions, upset by a nonlogical difference, open up beyond themselves. (Hollier 1992, 96)

Ne Le Benevole infatti il riferimento all'occhio pineale è identico a quello teorizzato da Bataille, ma dichiaratamente aperto sul nulla tradisce l'incapacità razionale di assorbire il trauma da parte di Aue:

Avevo la sensazione che il buco nella mia fronte si fosse aperto su un terzo occhio, un occhio pineale, non rivolto verso il sole, capace di contemplarne la luce accecante, ma diretto verso le tenebre, dotato del potere di guardare il volto nudo della morte, e di scorgerlo, quel volto, dietro a ogni viso di carne, sotto i sorrisi, attraverso la pelle più bianca e più sana, gli occhi più ridenti. (Littell 2007, 429)

Aue si sente in questo modo come «un puro sguardo o addirittura una cinepresa» (599), uno specchio opaco che riflette senza profondità le immagini del trauma al lettore. Questo determina inevitabilmente lo svilimento dell'immagine, come già sottolineato nei capitoli precedenti, immagine che venendo così meno al suo scopo testimoniale contribuisce a consolidare quello che Giglioli aveva definito il «lento apprendistato» all'indifferenza e all'assuefazione rispetto al trauma degli altri.

In Trittico. Tre studi da Francis Bacon Littell si era soffermato sulla rappresentazione dell'occhio nei dipinti del pittore irlandese. A proposito di quei soggetti che «in maniera strategica e disturbante» (Littell 2014,53$)$ impongono con forza «la propria indifferenza sull'intera scena che viene rappresentata sulla tela» (53) Littell ha utilizzato l'etichetta di «testimone indifferente» (53), definizione che sembra adattarsi alla figura di Aue ne Le Benevole: un testimone che «distoglie lo sguardo dalla sofferenza dell'altro, oppure guarda senza interesse» (53). È un passaggio che è possibile interpretare come la trasposizione letteraria di quella «crisis of witnessing» di cui hanno 
scritto Shoshana Felman e Dori Laub in Testimony: Crises of Witnessing in Literature, Psychoanalysis, and History (1991), nel senso che la portata del trauma di cui il narratore de Le Benevole cerca di dare memoria è tale da non permettere al testimone oculare di rimanere in contatto con la realtà di cui ha fatto esperienza.

The traumatic event, although real, took place outside the parameters of 'normal' reality, such as causality, sequence, place, and time. The trauma is thus an event that has no beginning, no ending, no before, no during and no after. This absence of categories that define it lends it to a quality of 'otherness', a salience, a timelessness and a ubiquity that puts it outside the range of associatively linked experiences, outside the range of comprehension, of recounting and of mastery. Trauma survivors live not with memories of the past, but with an event that could not and did not proceed through to its completion, has no ending, attained no closure, and therefore, as far as its survivors are concerned, continues into the present and is current in every respect. $(1991,69)$

Tirando le fila di quanto osservato fino a questo momento, è possibile leggere il significato del terzo occhio di Aue anche secondo un'altra prospettiva: quella del fallimento totale della volontà di rappresentare il trauma della Shoah rimanendo lucido, senza ammalarsi. Una crisi testimoniale non tanto nella prima generazione di testimoni, ma deficit interpretativo peculiare della generazione dell'inesperienza, delle non vittime, delle persone nate dopo la fine degli eventi della Shoah (Boswell 2012, 18-19): è in questi termini allora che l'episodio dimostrerebbe il collasso della finzione messa in scena dal narratore, il fallimento del tentativo di far coincidere l'invenzione romanzesca - strumento prediletto dalla generazione dei nuovi testimoni - con la narrazione della Storia. L'evento traumatico, nel tempo della postmemoria, sembrerebbe così destinato a sfuggire ancora. 


\section{Bibliografia}

Adorno, T.W. (1972). Prismi. Saggi sulla critica della cultura. Trad. di C. Mainoldi et al. Torino: Einaudi. Trad. di: Prismen. Kulturkritik und Gesellschaft. Frankfurt am Main: Suhrkamp Verlag, 1955.

Agamben, G. (1998). Quel che resta di Auschwitz. L'archivio e il testimone. Torino: Bollati Boringhieri.

Antelme, R. (1997). La specie umana. Trad. di Ginetta Vittorini. Torino: Einaudi. Trad. di: L'espèce humaine. Paris: Éditions Gallimard, 1957.

Arendt, H. (2001). La banalità del male. Eichmann a Gerusalemme. Trad. di Piero Bernardini. Milano: Feltrinelli. Trad. di: Eichmann in Jerusalem: A Report on the Banality of Evil. New York: Viking Press, 1963-64.

Bachtin, M. (2001). Estetica e romanzo. Trad. di Clara Strada Janovič. Torino: Einaudi. Trad. di: Voprosy literatury $i$ estetiki. Saint Petersburg: Khudozhestvennaya Literatura, 1975.

Baldini, A. (2012). «La memoria italiana della Shoah (1944-2009)». Scarpa, D. (a cura di), Atlante della letteratura italiana. Vol. 3: Dal romanticismo ad oggi. Torino: Einaudi, 758-63.

Baldini, A. (2014). «Primo Levi and the Italian Memory of the Shoah». Miscellanea, eds. Quest Editorial Staff, Quest. Issues in Contemporary Jewish History. Journal of Fondazione CDEC, July 7, 2014. http://www.quest-cdecjournal.it/focus.php? id=361.

Barenghi, M. (2013). Perché crediamo a Primo Levi? / Why Do We Believe Primo Levi?. Torino: Einaudi.

Barthes, R. (1997). «Sade-Pasolini». Toffetti, S. (a cura di), Sul cinema. Genova: il melangolo, 159-60. Ed. or., Le Monde, 16 giugno 1976.

Barthes R. [1978] (1984). "La Mécanique du charme». Calvino I., Le Chevalier inexistant. Paris: Seuil, 1-2.

Bataille, G. (1972). Critica dell'occhio. A cura di S. Finzi. Rimini: Guaraldi Editore. Trad. di saggi tratti da: Feuvres Complètes. Tomes I et II. Paris: Éditions Gallimard, 1970. 
Baudrillard, J. (1980). «Simulacri e fantascienza». Trad. di A. Caronia et al. Russo, L. (a cura di), La fantascienza e la critica. Testi del Convegno internazionale di Palermo. Milano: Feltrinelli, 52-7.

Baudrillard, J. (1993). L'illusione della fine o Lo sciopero degli eventi. Trad. di A. Serra. Milano: Anabasi. Trad. di: L'lllusion de la fin ou la grève des événements. Paris: Galilée, 1992.

Baudrillard, J. (2002). Lo spirito del terrorismo. Trad. di A. Serra. Milano: Raffaello Cortina. Trad. di: L'esprit du terrorism. Paris: Galilée, 2002.

Baudrillard, J. (2007). L'illusione dell'immortalità. Trad. di G. Biolghini. Roma: Armando. Trad. di: The vita illusion. New York: Columbia University Press, 2000.

Baudrillard, J. (2014). "Grande fratello». Codeluppi, V. (a cura di), Miti fatali. Twin Towers, Beaubourg, Disneyland, America, Andy Warhol, Michael Jackson, Guerra del Golfo, Madonna, Jeans, Grande Fratello. Milano: FrancoAngeli, 77-90. Trad. di: «Télémorphose». Télémorphose: précédé de L'élevage de poussière. Paris: Sens et Tonka, 2001.

Baudrillard, J. (2015). Lo scambio simbolico e la morte. Trad. di G. Mancuso. Milano: Feltrinelli. Trad. di: L'échange symbolique et la mort. Paris: Gallimard, 1976.

Bauman, Z. [1989] (2000). Modernity and the Holocaust. Ithaca: Cornell University Press.

Belpoliti, M. (2000-01). «Levi: il falso scandalo». La Rivista dei libri, 10(1), 25-7.

Benvenuti, G. (2012). Il romanzo neostorico italiano. Storia, memoria, narrazione. Roma: Carocci editore.

Berberich, C. (2018). «Introduction: The Holocaust in Contemporary Culture». Holocaust Studies, 25(1-2), 1-11. https://doi.org/10.1080/17504902. 2018.1472871.

Bergson, H. (1996). Materia e memoria: Saggio sulla relazione tra il corpo e lo spirito. A cura di A. Pessina. Roma-Bari: Laterza. Trad. di: Matière et mémoire. Essai sur la relation du corps à l'esprit. Paris: Presses universitaires de France, 1896.

Bernardini, P. et. al. (a cura di) (2006). La memoria del male. Percorsi tra glistermini del Novecento e il loro ricordo. Padova: Cleup.

Bettelheim, B. (2005). Sopravvivere e altri saggi. Trad. di A. Bottini. Milano: SE. Trad. di: Surviving and Other Essays. New York: Random House, 1979.

Bidussa, D. (2009). Dopo l'ultimo testimone. Torino: Einaudi.

Binet, L. (2011). HHhH. Il cervello di Himmler si chiama Heydrich. Trad. di M. Botto. Torino: Einaudi. Trad. di: HHhH. Paris: Éditions Grasset, 2010.

Blanrue, P.-É. (2006). Les Malveillantes, enquête sur le cas Jonathan Littell. Paris: Scali.

Boswell, M. (2012). Holocaust Impiety in Literature, Popular Music and Film. London: Palgrave Macmillan.

Broch, H. (1990). Il Kitsch. Trad. di R. Malagoli e S. Vertone. Torino: Einaudi. Trad. di: Philosophische Schriften 1. Kritik; Schriften zur Literatur 2. Theorie. Frankfurt am Main: Suhrkamp Verlag, 1975; 1977.

Brown, C.B. (2009). «The Difference Between History and Romance». Wieland, Ormond, Arthur Mevyn, and Edgar Huntly with Related Texts. Edited, with an Introduction and Notes by P. Barnard and S. Shapiro. Hackett, 341-3.

Burn, S.J. (2007). «The Collapse of Everything: William Gaddis and the Encyclopedic Novel». Tabbi, J.; Shavers, R. (eds), Paper Empire: William Gaddis and the World System. Tuscaloosa: University of Alabama Press, 46-62. 
Calvino, I. [1964] (2002). «Prefazione». Il sentiero dei nidi di ragno. 2a ed. Milano: Mondadori, V-XXV.

Calvino, I. (2007). "Sade è dentro di noi (Pasolini, Salò)». Barenghi, M. (a cura di), Saggi, vol. 2. Milano: Mondadori, 1933. Ed. or., Corriere della Sera, 30 novembre 1975.

Carrère, E. (2014). Limonov. Trad. di F. Bergamasco. Milano: Adelphi. Trad. di: Limonov. Paris: P.O.L., 2011.

Caruth, C. (1996). Unclaimed Experience: Trauma, Narrative and History. Baltimora: JHU.

Cavaglion, A. (2008). «Primo Levi, il 1938, il fascismo e la storia d'Italia». Belfagor. Rassegna di varia umanità, 63(6), 719-23.

Cercas, J. (2002). Soldati di Salamina. Trad. di P. Cacucci. Milano: Guanda. Trad. di: Soldados de Salamina. Barcelona: Tusquets, 2001.

Cercas, J. (2015). L'impostore. Trad. di B. Arpaia. Parma: Guanda. Trad. di: El impostor. Barcelona: Literatura Random House, 2014.

Césaire, A. [1999] (2010). Discorso sul colonialismo seguito da Discorso sulla negritudine. Trad. di L. Di Genio. Verona: Ombre Corte. Trad. di: Discours sur le colonialisme. Paris: Réclame, 1950.

Cinquegrani, A. (2018). Il sacrificio di Bess. Sei immagini su nazismo e contemporaneità. Milano; Udine: Mimesis.

Cinquegrani, A. (2019a). «ll ritorno a casa secondo Primo Levi». Cinquegrani, A.; Crotti, I. (a cura di), «Un viaggio realmente avvenuto». Studi in onore di Ricciarda Ricorda. Venezia: Edizioni Ca' Foscari, 331-44. https://phaidra. cab.unipd.it/o:450839.

Cinquegrani, A. (2019b). «Imprese e letteratura». Cinquegrani, A. (a cura di), Imprese letterarie. Venezia: Edizioni Ca' Foscari, 15-43. http://doi. org/10.30687/978-88-6969-356-4/001.

Cinquegrani, A. (2020). «Alterità e inesperienza nell'opera di Don DeLillo». Regazzoni, S.; Domínguez Gutiérrez, M.C. (a cura di), L'altro sono io / El otro soy io. Scritture plurali e letture migrante / Escrituras plurales y lecturas migrantes. Venezia: Edizioni Ca' Foscari, 249-62. http://doi.org/10.30687/97888-6969-396-๑/०20.

Coffman, C.K. (2015). «Introduction: Lonely Atoms». Coffmann, Lukes 2015, 1-22.

Coffmann, C.K.; Lukes, D. (eds) (2015). William T. Vollmann. A Critical Companion. Newark: University of Delaware Press.

Cole, T. (2000). Selling the Holocaust: From Auschwitz to Schindler. How History is Bought, Packaged, and Sold. New York; London: Routledge.

Currie, M. (1998). Postmodern Narrative Theory. Basingstoke: Macmillan.

de Certeau, M. [1977] (2006). La scrittura della storia. Trad. di A. Jeronimidis. Milano: Jaca Book. Trad. di: L'Écriture de l'histoire. Paris: Éditions Gallimard, 1975.

DeLillo, D. (1999). White Noise. New York: Penguin.

DeLillo, D. (2000). Underworld. Trad. it. di D. Vezzoli. Torino: Einaudi. Trad. di: Underworld. New York: Scribner, 1997.

DeLillo, D. (2005). Running Dog. Trad. di S. Pareschi. Torino: Einaudi. Trad. di: Running Dog. New York: Knopf, 1978.

DeLillo, D. (2014). Rumore bianco. Trad. di M. Biondi. Torino: Einaudi. Trad. di: White Noise. New York: Viking, 1985.

Dick, P.K. (2019). La svastica sul sole. Trad. di M. Nati. Roma: Fanucci. Trad. di: The Man in the High Castle. New York: Putnam's Sons, 1962. 
Doležel, L. (1999). Heterocosmica. Fiction e mondi possibili. Trad. di M. Botto. Milano: Bompiani. Trad. di: Heterocosmica. Fiction and Possible Worlds. Baltimore: The Johns Hopkins University Press, 1998.

Domenichelli, M. (2011). Lo scriba e l'oblio. Letteratura e storia: teoria e critica delle rappresentazioni nell'epoca borghese. Pisa: Edizioni ETS.

Donnarumma, R. (2014). Ipermodernità: dove va la narrativa contemporanea. Bologna: il Mulino.

Eco, U. (1964). Apocalittici e integrati. Comunicazioni di massa e teorie della cultura di massa. Milano Bompiani.

Eco, U. (1984). «Postille al Nome della rosa». Il nome della rosa. Milano: Bompiani, 528-33.

Elias, A. (2001). Sublime Desire. History and Post-1960s Fiction. Baltimore: John Hopkins University Press.

Eliot, T.S. (1923). «Ulysses, Order and Myth». The Dial, 75, 480-3.

Ellis, J. (1999). Seeing Things: Television in the Age of Uncertainty. London: I.B. Tauris.

Ercolino, S. (2015). Il romanzo massimalista. Milano: Bompiani.

Felman, S.; Laub, D. (1991). Testimony: Crises of Witnessing in Literature, Psychoanalysis, and History. New York; London: Routledge.

Finkelstein, N. (2000). The Holocaust Industry. Reflections on the Exploitation of Jewish Suffering. New York; London: Verso.

Foster, H. (2006). Il ritorno del reale: l'avanguardia alla fine del Novecento. Trad. di B. Carneglia. Milano: Postmedia. Trad. di: The Return of the Real: The Avant-Garde at the End of the Century. Cambridge (MA): MIT Press, 1996.

Freud, S. [1932] (1971). «Perché la guerra». Il disagio della civiltà e altri saggi. Torino: Boringhieri, 283-95.

Freud, S. [1905] (1988). «Tre saggi sulla teoria sessuale (1905)». La sessualità. Trad. it. di I. Valent. Milano: Mondadori, 23-139.

Frye, N. (1969). Anatomia della critica. Teoria dei modi, dei simboli, dei miti e dei generi letterari. Trad. di P. Rosa-Clot e S. Stratta. Torino: Einaudi. Trad. di: Anatomy of Criticism. Four Essays. Princeton: Princeton University Press, 1957.

Frye, N. (1978). La scrittura secolare. Studio sulla scrittura del «romance». Trad. it. di A. Lorenzini. Bologna: il Mulino. Trad. di: The Secolar Scripture. A Study of the Structure of Romance. Harvard: Harvard University Press, 1976.

Fukuyama, F. (1992). La fine della storia e l'ultimo uomo. Trad. di D. Ceni. Milano: Rizzoli. Trad. di: The End of History and the Last Man. New York: Free Press, 1992.

Gentili-Tedeschi, E. et. al. (1999). Primo Levi testimone e scrittore di storia. Giornate distudio in ricordo di Primo Levi (1997 Saint-Vincent). Istituto storico della Resistenza in Valle d'Aosta. Firenze: Giuntina.

Gheno, V. (2019). Potere alle parole: perché usarle meglio. Torino: Einaudi.

Giglioli, D. (2011). Senza trauma. Scrittura dell'estremo e narrativa del nuovo millennio. Macerata: Quodlibet.

Giglioli, D. (2012). «ll buco e l'evento. Sul Taccuino siriano di Jonathan Littell». Schiavini Trezzi, J. (a cura di), Mosaico francese. Studi in onore di Alberto Castoldi. Bergamo: Moretti \& Vitali, 279-91.

Giglioli, D. (2014). Critica della vittima. Un esperimento con l'etica. Milano: Nottetempo.

Gordon, R.S.C. (2006). «Which Holocaust? Primo Levi and the field of Holocaust Memory in Post-War Italy». Italian Studies, 61(1), 85-113. 
Guez, O. (2018). La scomparsa di Josef Mengele. Trad. di M. Botto. Vicenza: Neri Pozza. Trad. di: La disparition de JosefMengele. Paris: Grasset \& Fasquelle 2017.

Halbwachs, M. (1987). La memoria collettiva. A cura di P. Jedlowski. Milano: UNICOPLI.

Hilberg, R. (1995). La distruzione degli Ebrei d'Europa. 3 voll. Trad. di F. Sessi e G. Guastalla. Torino: Einaudi. Trad. di: The Destruction of the European Jews. New York; London: Holmes \& Meier, 1985.

Hirsch, M. (2012). The Generation of Postmemory: Writing and Visual Culture After the Holocaust. New York: Columbia University Press.

Hollier, D. (1992). Against Architecture: The Writings of Georges Bataille. Cambridge: MIT Press.

Hutcheon, L. (1988). A Poetics of Postmodernism. History, Theory, Fiction. New York; London: Routledge.

Jameson, F. (2007). Postmodernismo, ovvero La logica culturale del tardo capitalismo. Trad. di M. Manganelli. Roma: Fazi Editore. Trad. di:Postmodernism, or, the Cultural Logic of Late Capitalism. Durham: Duke University Press, 1991.

Janeczek, H. (2017). La ragazza con la Leica. Milano: Guanda.

Jesi, F. (1968). Letteratura e mito. Torino: Einaudi.

Jung, C.G. (1965). Opere complete. Vol. 5, Simboli della trasformazione. A cura di L. Aurigemma. Torino: Bollati Boringhieri.

Jung, C.G. (1969). Tipi psicologici. Trad. di C.L. Musatti e L. Aurigemma. Torino: Boringhieri.

Jung, C.G. (1985). «Picasso». Opere. Vol. 10.1, Civiltà in transizione - Il periodo tra le due guerre. Trad. di P. Santarcangeli. Torino: Boringhieri, 405-12. Trad. di: «Picasso». Neue Zürcher Zeitung, 13 novembre 1932.

Kiš, D. (2009). Homo poeticus. Saggi e interviste. Trad. di D. Badnjevič. Milano: Adelphi. Trad. di: Sontag, S. (ed.), Homo poeticus. New York: Farrar, Straus and Groux, 1995.

Klemperer, V. [1947] (2000). The Language of the Third Reich. LTI: Lingua Tertii Imperii. A Philologist's Notebook. London; New Brunswick: Transaction Publishers.

Kristeva, J. (1988). Sole nero. Depressione e melanconia. Trad. di A. Serra. Milano: Feltrinelli. Trad. di: Soleil noir. Dépression et mélancolie. Paris: Éditions Gallimard, 1987.

Kristeva, J. (2007). «À propos des Bienveillantes. De l'abjection à la banalité du mal». L'Infini. Littérature, Philosophie, Art, Science, Politique, 99, 22-35.

Kuon, P. (2012). "From "Kitsch" to "Splatter". The Aesthetics of Violence in The Kindly Ones». Barjonet, A.; Razinsky, L. (eds), Writing the Holocaust Today. Critical Perspectives on Jonathan Littell's "The Kindly Ones". Amsterdam; New York: Rodopi, 33-45.

Lanzmann, C. (1995). «The Obscenity of Understanding: An Evening With Claude Lanzmann». Caruth, C. (ed.), Trauma: Explorations in Memory. Baltimore: Johns Hopkins University Press, 473-95.

Léon-Dufour, X. (1978). Dizionario del Nuovo Testamento. Trad. di P. Crespi. Brescia: Queriniana. Trad. di: Dictionnaire du Nouveau Testament. Paris: Éditions du Seuil, 1975.

Levi, P. [1958] (2016). Se questo è un uomo. Belpoliti, M. (a cura di), Opere complete, vol. 1. Torino: Einaudi, 135-304.

Levi, P. [1963] (2016). La tregua. Belpoliti, M. (a cura di), Opere complete, vol. 1. Torino: Einaudi, 305-473. 
Levi, P. [1966] (2016). Storie naturali. Belpoliti, M. (a cura di), Opere complete, vol. 1. Torino: Einaudi, 475-651.

Levi, P. [1975] (2016). Il sistema periodico. Belpoliti, M. (a cura di), Opere complete, vol. 1. Torino: Einaudi, 857-1032.

Levi, P. [1982] (2016). Se non ora, quando?. Belpoliti, M. (a cura di), Opere complete, vol. 2. Torino: Einaudi, 413-674.

Levi, P. [1986] (2016). I sommersi e i salvati. Belpoliti, M. (a cura di), Opere complete, vol. 2. Torino: Einaudi, 1143-276.

Levi, P. [1997] (2018). Opere complete. Conversazioni, interviste e dichiarazioni, vol. 3. A cura di M. Belpoliti. Torino: Einaudi.

Levi, P.; Camon, F. (1987). Autoritratto di Primo Levi. Padova: Nord-Est.

Liebtag, M. (2015). «Our Oriental Heritage: Seeking the Postcolonial Postmodern in William T. Vollmann's You Bright and Risen Angels». Coffmann, Lukes 2015, 191-208.

Lipstadt, D.E. (2014). Il processo Eichmann. Trad. di M.L. Chiesara. Torino: Einaudi. Trad di: The Eichmann Trial. New York: Schocken Books, 2011.

Littell, J. (2007). Le Benevole. Trad. di M. Botto. Torino: Einaudi. Trad. di: Les Bienveillantes. Paris: Éditions Gallimard, 2006.

Littell, J. (2009). Il secco e l'umido. Una breve incursione in territorio fascista. Trad. di M. Botto. Torino: Einaudi. Trad. di: Le sec et l'humide. Une brève incursion en territoire fasciste. Paris: Éditions Gallimard, 2008.

Littell, J. (2014). Trittico. Tre studi da Francis Bacon. Trad. di L. Bianco. Torino: Einaudi. Trad. di: Triptyque. Trois études sur Francis Bacon. Paris: Éditions Gallimard, 2010.

Loshitzky, Y. (ed.) (1997). Spielberg's Holocaust. Critical Perspectives on Schindler's List. Bloomington: Indiana University Press.

Lyotard, J.-F. (1981). La condizione postmoderna. Rapporto sul sapere. Trad. di C. Formenti. Milano: Feltrinelli. Trad. di: La Condition postmoderne. Rapport sur le savoir. Paris: Les Éditions de Minuit, 1979.

Lyotard, J.-F. (1987). Il postmoderno spiegato ai bambini. Trad. di A. Serra. Milano: Feltrinelli. Trad. di: Le Postmoderne expliqué aux enfants. Paris: Galilee, 1986.

Malvestio, M. (2019). The Conflict Revisited. Representing the Second World War in Twenty-First Century Fiction [tesi di dottorato]. Padova: Università degli Studi di Padova.

Manchel, F. (1995). «A Reel Witness: Steven Spielberg's Representation of the Holocaust in Schindler's List». The Journal of Modern History, 67(1), 83-100.

Mazzarella, A. (2017). Le relazioni pericolose. Sensazioni e sentimenti del nostro tempo. Torino: Bollati Boringhieri.

Mazzoni, G. (2011). Teoria del romanzo. Bologna: il Mulino.

McHale, B. (1987). Postmodernist Fiction. New York; London: Routledge.

Meghnagi, D. (a cura di) (2007). Memoria della Shoah. Dopo «i testimoni». Roma: Donizelli.

Mercier-Leca, F. (2007). «Les Bienveillantes et la tragédie grecque: une suite macabre à L'Orestie d'Eschyle». Le Débat, 144(2), 45-55.

Moretti, F. (1994). Opere mondo. Saggio sulla forma epica dal "Faust" a "Cent'anni di solitudine". Torino: Einaudi.

Nolte, E.; Rusconi, G.E. (1987). Germania, un passato che non passa: icrimini nazisti e l'identità tedesca. Torino: Einaudi.

Novick, P. (1999). The Holocaust in American Life. Boston: Houghton Mifflin.

Parente, M. (2018). Il più grande artista del mondo dopo Adolf Hitler. Milano: Giunti. Ebook. 
Pasolini, P.P. [1975] (2001). «/l sesso come metafora del potere». Pasolini, P.P., Per il cinema. A cura di W. Siti. Milano: Mondadori, 2063-7.

Pasolini, P.P. [1979] (2001). «De Sade e l'universo dei consumi». Pasolini, P.P., Per il cinema. A cura di W. Siti. Milano: Mondadori, 3019-22.

Petrella, A. (2006). «Dal postmoderno al romanzo epico. Linee per la letteratura italiano dell'ultimo Novecento». Allegoria, 52-53, 134-48.

Piga Bruni, E. (2018). La lotta e il negativo. Sul romanzo storico contemporaneo. Milano: Mimesis.

Pirandello, L. [1925] (2016). «Prefazione» a Sei personaggi in cerca d'autore. Gibellini, P. (a cura di), Il meglio del teatro. Drammi scelti. Milano: BUR, 327-38.

Pynchon (2017). L'arcobaleno della gravità. Trad. di G. Natale. Milano: BUR. Trad. di: Gravity's Rainbow. New York: Viking, 1973.

Razinsky, L. (2010). «Not the Witness We Wished For: Testimony in Jonathan Littell's Kindly Ones». Modern Language Quarterly, 71(2), 175-96.

Recchia Luciani, F.R.; Vercelli, C. (a cura di) (2016). Pop Shoah? Immaginari del genocidio ebraico. Genova: Il Melangolo.

Reeve, C. (1785). The Progress of Romance. Colchester: Keymer.

Rosenbaum, A.S. (ed.) (1996). Is the Holocaust Unique? Perspective on Comparative Genocide. Boulder: Westview Press.

Rosenfeld, A.H. (2002). «The Assault on Holocaust Memory». KulturPoetik, 2(1), 82-101.

Roth, P. (2014). Il complotto contro l'America. Trad. di V. Mantovani. Einaudi: Torino. Trad. di: The Plot against America. Boston: Houghton Mifflin, 2004.

Rothberg, M. (2009). Multidirectional Memory: Remembering the Holocaust in the Age of Decolonization. Stanford: Stanford University Press.

Sandberg, E. (2014). «'This Incomprehensible Thing': Jonathan Littell's The Kindly Ones and the Aesthetics of Excess». The Cambridge Quarterly, 43(3), 231-55.

Santin, B.M. (2015). «Kurt Gerstein and the Tragic Parable of 'Clean Hands': The Imaginative Role of Fiction in the Moral Calculus of William T. Vollmann». Coffmann, Lukes 2015, 141-66.

Scaffai, N. (2013). "Jonathan Littell. Il secco e l'umido. Una breve incursione in territorio fascista». Allegoria, 61, 221.

Scarpa, D. (2010). Storie avventurose di libri necessari. Roma: Gaffi Editore.

Schaller, D.J.; Zimmerer, J. (eds) (2009). The Origins of the Genocide: Raphael Lemkin as a Historian of Mass Violence. London; New York: Routledge.

Schields, D. (2010). Reality Hunger: A Manifesto. New York: Knopf.

Scott, W. (1834). Essay on Romance. London: Encyclopaedia Britannica.

Scott, W. [1834] (1983). Essay on Romance. London: Encyclopaedia Britannica. Trad. it. in S. Perosa (a cura di), Teorie inglesi del romanzo, 1700-1900. Milano: Bompiani, 200.

Scurati, A. (2006). La letteratura dell'inesperienza. Scrivere romanzi al tempo della televisione. Milano: Bompiani.

Scurati, A. (2012). Dal tragico all'osceno. Raccontare la morte nel XXI secolo. Milano: Bompiani.

Scurati, A. (2014). «Europa, zona di guerra. Il senso tragico della storia e la rinascita del romanzo francese». Fictions. Studi sulla narratività, XIII, 89-97.

Scurati, A. (2018). M. Il figlio del secolo. Milano: Giunti.

Simonetti, G. (2018). La letteratura circostante. Narrativa e poesia nell'Italia contemporanea. Bologna: il Mulino. 
Sorcinelli, P. (a cura di) (1987). Lavoro, criminalità e alienazione mentale. Ricerche sulle Marche tra Otto e Novecento. Ancona: Il Lavoro Editoriale.

Steiner, G. (1988). «The Long Life of Metaphor. An Approach to 'the Shoah'». Lang, B. (ed.), Writing and the Holocaust. New York; London: Holmes \& Meier, 154-71.

Sullam Calimani, A.-V. (2001). I nomi dello sterminio. Torino: Einaudi.

Tal, K. (1996). Worlds of Hurt: Reading the Literature of Trauma. Cambridge: Cambridge University Press.

Theweleit, K. (1977-78). Männerphantasien. 2 voll. Frankfurt am Main: Stroemfeld.

Theweleit, K.; Nunan, T. (2009). «On the German Reaction to Jonathan Littell's Les Bienveillantes». New German Critique, 106, 21-34.

Tirinanzi de Medici, C. (2018). Il romanzo italiano contemporaneo. Dalla fine degli anni Settanta a oggi. Roma: Carocci.

Todorov, T. (2007). «Prefazione». Levi, P., I sommersie isalvati. Torino: Einaudi, I-XI.

Traverso, E. (2004). Auschwitz e gli intellettuali. La Shoah nella cultura del dopoguerra. Bologna: il Mulino.

Vattimo, G. (1985). La fine della modernità. Milano: Garzanti.

Vogler, C. (2005). Il viaggio dell'eroe. Roma: Audino.

Vollmann, W.T. (1992). «American Writing Today: A Diagnosis of the Disease». Conjunctions, 15, s.p. http://www.conjunctions.com/print/article/william-t-vollmann-c15.

Vollmann, W.T. (2003). Rising Up and Rising Down: Some Thoughts on Violence, Freedom and Urgent Means. 7 vols. San Francisco: McSweeney's.

Vollmann, W.T. (2010). Europe Central. Trad. di G. Pannofino. Milano: Mondadori. Trad. di: Europe Central. New York: Viking Press, 2005.

Wallace, D.F. (1999). «E Unibus Pluram: gli scrittori americani e la televisione». Tennis, $t v$, trigonometria, tornado (e altre cose divertenti che non farò mai più). Trad. di V. Ostuni et al. Roma: Minimum Fax, 29-104. Trad. di: «E Unibus Pluram: television and U.S. fiction». A Supposedly Fun Thing I'll Never Do Again. New York: Little, Brown and Company, 1997, 21-83.

White, H. (1978). Retorica e storia. 2 voll. Trad. di P. Vitulano. Napoli: Guida Editori. Trad. di: Metahistory. The Historical Imagination in Nineteenth-Century Europe. Baltimore: Johns Hopkins University Press, 1973.

White, H. (2006). «ll testo storico come artefatto letterario» in Forme di storia. Dalla realtà alla narrazione. A cura di E. Tortarolo. Roma: Carocci. Trad. di: «The Historical Text as Literary Artifact». Tropics of Discourse: Essays in Cultural Criticism. Baltimore: Johns Hopkins University Press, 1978.

Wieviorka, A. (1999). L'era del testimone. Trad. di F. Sossi. Milano: Raffaello Cortina Editore. Trad. di: L'Ère du témoin. Paris: Plon, 1998.

Wyman, D.S.; Rosenzveig, C.H. (eds) (1996). The World Reacts to the Holocaust. Baltimore: John Hopkins University Press.

Zanotti, P. (1998). Il modo romanzesco. Roma-Bari: Laterza.

Žižek, S. (2001). Enjoy your Symptom! Jacques Lacan in Hollywood and Out. New York; London: Routledge.

Žižek, S. (2002). Benvenuti nel deserto del reale. Cinque saggi sull'11 settembre e date simili. Trad. di P. Vereni. Milano: Meltemi. Trad. di: Welcome to the Desert of the Real. Five Essays on September 11 and Related Dates. London; New York: Verso Books, 2002. 

Da molti anni le narrazioni sulla Shoah si diffondono sempre più. Esistono motivazioni etiche per questa crescente attenzione. Ma forse non sono le sole. Questo libro indaga le strutture narrative sottese a queste storie per verificare se rispondano alle esigenze dei lettori. Ne emerge un quadro preoccupante nel quale l'inaspettata liaison tra romance e Shoah rischia di trasformare la narrazione storica di un evento tragico - e tragico fino all'ineffabile - in un genere letterario prossimo all'intrattenimento.

Il volume affronta questioni teoriche - la ricezione, il romance, il postmoderno - e casi specifici tratti dalla grande letteratura su questi temi - Primo Levi, Littell, Vollman, Cercas - per interrogarsi su cosa siano le narrazioni sulla Shoah oggi e cosa rischino di diventare, dopo l'ultimo testimone.

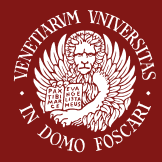

Università

Ca'Foscari

Venezia
} 